\title{
Binomial Difference Ideal and Toric Difference Variety*
}

\author{
Xiao-Shan Gao, Zhang Huang, Chun-Ming Yuan \\ KLMM, Academy of Mathematics and Systems Science \\ Chinese Academy of Sciences, Beijing 100190, China \\ $\{$ xgao,cmyuan\}@mmrc.iss.ac.cn
}

\begin{abstract}
In this paper, the concepts of binomial difference ideals and toric difference varieties are defined and their properties are proved. Two canonical representations for Laurent binomial difference ideals are given using the reduced Gröbner basis of $\mathbb{Z}[x]$-lattices and regular and coherent difference ascending chains, respectively. Criteria for a Laurent binomial difference ideal to be reflexive, prime, well-mixed, perfect, and toric are given in terms of their support lattices which are $\mathbb{Z}[x]$-lattices. The reflexive, well-mixed, and perfect closures of a Laurent binomial difference ideal are shown to be binomial. Four equivalent definitions for toric difference varieties are presented. Finally, algorithms are given to check whether a given Laurent binomial difference ideal $\mathcal{I}$ is reflexive, prime, well-mixed, perfect, or toric, and in the negative case, to compute the reflexive, wellmixed, and perfect closures of $\mathcal{I}$. An algorithm is given to decompose a finitely generated perfect binomial difference ideal as the intersection of reflexive prime binomial difference ideals.
\end{abstract}

Keywords. Binomial difference ideal, toric difference variety, difference torus, $\mathbb{Z}[x]$ lattice, difference characteristic set, decomposition of perfect binomial difference ideal.

Mathematics Subject Classification [2000]. Primary 12H10, 14M25; Secondary 14Q99, 68W30.

\section{Contents}

1 Introduction 2

2 Preliminaries about difference algebra

2.1 Difference polynomial and Laurent difference polynomial . . . . . . . . . . . 6

2.2 Characteristic set for a difference polynomial system . . . . . . . . . . . G

\footnotetext{
*Partially supported by a National Key Basic Research Project of China (2011CB302400) and by grants from NSFC (60821002,11101411).
} 
$3 \mathbb{Z}[x]$-lattice 9

3.1 Gröbner basis and generalized Hermite normal form . . . . . . . . . . 9 9

3.2 Kernel of a matrix in $\mathbb{Z}[x]^{n \times s} \ldots \ldots \ldots \ldots \ldots \ldots$

4 Laurent binomial $\sigma$-ideal 14

4.1 Laurent binomial $\sigma$-ideal . . . . . . . . . . . . . . . . . . . . . . 14

4.2 Characteristic set of Laurent binomial $\sigma$-ideal . . . . . . . . . . . . . . 16

4.3 Partial character and Laurent binomial $\sigma$-ideal . . . . . . . . . . . . . . 20

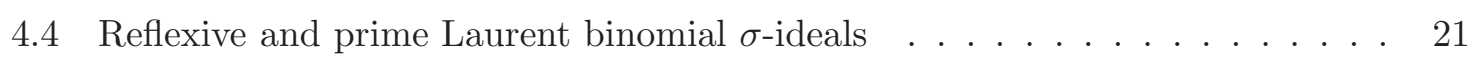

4.5 Well-mixed and perfect Laurent binomial $\sigma$-ideals . . . . . . . . . . . . . 26

5 Binomial $\sigma$-ideal 32

5.1 Basic properties of binomial $\sigma$-ideal . . . . . . . . . . . . . . 32

5.2 Normal binomial $\sigma$-ideal . . . . . . . . . . . . . . . . . . . 35

5.3 Characteristic set for normal binomial $\sigma$-ideal . . . . . . . . . . . . . . 39

5.4 Perfect closure of binomial $\sigma$-ideal and binomial $\sigma$-variety . . . . . . . . . . 42

6 Toric $\sigma$-ideal and toric $\sigma$-variety 45

6.1 Toric $\sigma$-variety . . . . . . . . . . . . . . . . . . . 46

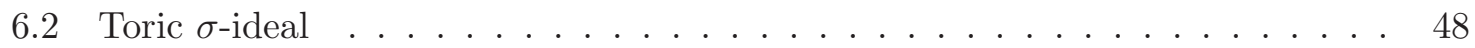

$6.3 \sigma$-torus and toric $\sigma$-variety in terms of group action . . . . . . . . . . 51

$6.4 \sigma$-Chow form and order of toric $\sigma$-variety . . . . . . . . . . . . 54

7 Algorithms for $\mathbb{Z}[x]$-lattice and binomial $\sigma$-ideal 5

$7.1 \quad x$-saturation of $\mathbb{Z}[x]$-lattice . . . . . . . . . . . . . . 56

$7.2 \mathbb{Z}$-saturation of $\mathbb{Z}[x]$-lattice . . . . . . . . . . . . . . 58

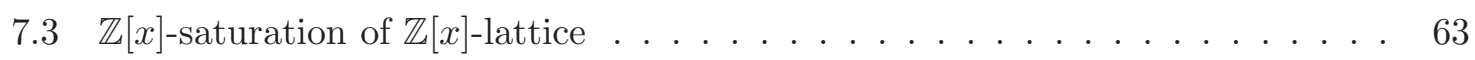

7.4 Algorithms for binomial $\sigma$-ideals $\ldots \ldots \ldots \ldots \ldots$

8 Conclusion $\quad 70$

\section{Introduction}

The theory of toric varieties has been extensively studied since its foundation in the early 1970s by Demazure [6], Miyake-Oda [27], Mumford et al. [18, and Satake [31], due to its deep connections with polytopes, combinatorics, symplectic geometry, topology, and its applications in physics, coding theory, algebraic statistics, and hypergeometric functions [5, 8, 13, 28. Toric varieties are often used as an effective testing ground for general theories of algebraic geometry. In [7, Eisendbud and Sturmfels initiated the study of binomial ideals 
which enriched the algebraic aspects of toric varieties and leaded to more applications [26, 29,

In this paper, we initiate the study of binomial difference ideals and toric difference varieties and hope that they will play similar roles in difference algebraic geometry to their algebraic counterparts in algebraic geometry. Difference algebra and difference algebraic geometry were founded by Ritt [30] and Cohn [3], who aimed to study algebraic difference equations as algebraic geometry to polynomial equations. Besides the early achievements, some of the major recent advances in this field include the difference Galois theory and its applications 32, the establishment of the theory of difference scheme and the proof of the Jacobi bound for difference equations [15], and the explicit description of invariant varieties under coordinatewise difference operators defined with univariate polynomials [25].

We now describe the main results of this paper. In Section $[3$, we prove basic properties of $\mathbb{Z}[x]$-lattices. By a $\mathbb{Z}[x]$-lattice, we mean a $\mathbb{Z}[x]$-module in $\mathbb{Z}[x]^{n}$. $\mathbb{Z}[x]$-lattices play the same role as $\mathbb{Z}$-lattices in the study of binomial ideals and toric varieties. Here, $x$ is used to denote the difference operator $\sigma$. For instance, $a^{3} \sigma(a)^{2}$ is denoted as $a^{2 x+3}$. Many properties of binomial difference ideals can be described or proved with the help of $\mathbb{Z}[x]$-lattices. Since $\mathbb{Z}[x]$ is not a PID, the Hermite normal form for a matrix with entries in $\mathbb{Z}[x]$ does not exist. In this section, we introduce the concept of generalized Hermite normal form and show that a matrix is a generalized Hermite normal form if and only if its columns form a reduced Gröbner basis for a $\mathbb{Z}[x]$-lattice. In general, a $\mathbb{Z}[x]$-lattice is not a free $\mathbb{Z}[x]$-module. We prove that the kernel of a matrix with entries in $\mathbb{Z}[x]$ is a free $\mathbb{Z}[x]$ module, which plays a key role in the study of toric difference varieties.

In Section 4, we prove basic properties of Laurent binomial difference ideals. Gröbner bases play an important role in the study of binomial ideals [7]. In general, a binomial difference ideal is not finitely generated and does not have a finite Gröbner basis. Instead, the theory of characteristic set for difference polynomial systems [10] is used for similar purposes. In Sections 4.2 and 4.3, we give canonical forms for Laurent binomial difference ideals in terms of $\mathbb{Z}[x]$-lattices and characteristic sets. Let $\mathcal{F}$ be a difference field with a difference operator $\sigma, \mathcal{F}^{*}=\mathcal{F} \backslash\{0\}, \mathbb{Y}=\left\{y_{1}, \ldots, y_{n}\right\}$ a set of difference indeterminates, and $\mathcal{F}\left\{\mathbb{Y}^{ \pm}\right\}$the ring of Laurent difference polynomials in $\mathbb{Y}$. Then

Theorem 1.1 $\mathcal{I} \subset \mathcal{F}\left\{\mathbb{Y}^{ \pm}\right\}$is a proper Laurent binomial difference ideal if and only if

(1) $\mathcal{I}=[\mathcal{A}]$, where $\mathcal{A}=\mathbb{Y}^{\mathbf{f}_{1}}-c_{1}, \ldots, \mathbb{Y}^{\mathbf{f}_{s}}-c_{s}, \mathbf{f}_{i} \in \mathbb{Z}[x]^{n}, c_{i} \in \mathcal{F}^{*}, \mathbb{f}=\left\{\mathbf{f}_{1}, \ldots, \mathbf{f}_{s}\right\}$ is a reduced Gröbner basis of a $\mathbb{Z}[x]$-lattice, and $[\mathcal{A}] \neq[1]$.

(2) $\mathcal{I}=[\mathcal{A}]$, where $\mathcal{A}=\mathbb{Y}^{\mathbf{f}_{1}}-c_{1}, \ldots, \mathbb{Y}^{\mathbf{f}_{s}}-c_{s}, \mathbf{f}_{i} \in \mathbb{Z}[x]^{n}, c_{i} \in \mathcal{F}^{*}$, and $\mathcal{A}$ is a regular and coherent difference ascending chain.

(3) $\mathcal{I}=\mathcal{I}(\rho)=\left[\mathbb{Y}^{\mathbf{f}}-\rho(\mathbf{f}) \mid \mathbf{f} \in L_{\rho}\right]$, where $\rho$ is a homomorphism from a $\mathbb{Z}[x]$-lattice $L_{\rho}$ generated by $\mathbb{f}$ to the multiplicative group $\mathcal{F}^{*}$ satisfying $\rho\left(\mathbf{f}_{i}\right)=c_{i}$ and $\rho(\sigma(\mathbf{f}))=\sigma(\rho(\mathbf{f}))$ for $\mathbf{f} \in L_{\rho}$.

In (1) and (2), $\mathcal{A}$ is a characteristic set of the difference ideal $\mathcal{I}$.

In Sections 4.4 and 4.5, we give criteria for a Laurent binomial difference ideal to be prime, reflexive, well-mixed, and perfect in terms of its lattice support. 
Theorem 1.2 Let $\mathcal{I}$ be a proper Laurent binomial difference ideal and $L=\left\{\mathbf{f} \mid \mathbb{Y}^{\mathbf{f}}-c_{\mathbf{f}} \in \mathcal{I}\right\}$ the support lattice of $\mathcal{I}$. If $\mathcal{F}$ is algebraically closed and inversive, then

(1) $\mathcal{I}$ is prime if and only if $L$ is $\mathbb{Z}$-saturated, that is, $k \mathbf{f} \in L$ implies $\mathbf{f} \in L$ for $k \in \mathbb{N}$ and $\mathbf{f} \in \mathbb{Z}[x]^{n}$.

(2) $\mathcal{I}$ is reflexive if and only if $L$ is $x$-saturated, that is, $\mathbf{f}^{x} \in L$ implies $\mathbf{f} \in L$ for $\mathbf{f} \in \mathbb{Z}[x]^{n}$.

(3) If the well-mixed closure of $\mathcal{I}$ is not [1], then $\mathcal{I}$ is well-mixed if and only if $L$ is $\mathrm{M}$ saturated, that is, $k \mathbf{f} \in L$ implies $\left(x-o_{k}\right) \mathbf{f} \in L$ for $k \in \mathbb{N}$ and $\mathbf{f} \in \mathbb{Z}[x]^{n}$, where $o_{k} \in \mathbb{N}$ is a number determined by the difference field $\mathcal{F}$.

(4) If the perfect closure $\{\mathcal{I}\}$ of $\mathcal{I}$ is not [1], then $\mathcal{I}$ is perfect if and only if $L$ is M-saturated and $x$-saturated.

The criterion for prime ideals is similar to the algebraic case, but the criteria for reflexive, well-mixed, and perfect difference ideals are unique to difference algebra and are first proposed in this paper. In particular, the criterion for well-mixed difference ideals is quite intriguing.

Based on the above theorem, it is shown that the reflexive, well-mixed, and perfect closures of a Laurent binomial difference ideal $I$ with support lattice $L$ are still binomial, whose support lattices are the $x_{-}, M$-, and the $x$ - $M$-saturation lattice of $L$, respectively. It is further shown that any perfect Laurent binomial difference ideal $\mathcal{I}$ can be written as the intersection of Laurent reflexive prime binomial difference ideals whose support lattices are the $x$-Z्Z- saturation of the support lattice of $\mathcal{I}$. Since difference polynomial rings are not Notherian, binomial difference ideals do not have an analog of the primary decomposition theorem in the algebraic case.

In Section 5, binomial difference ideals are studied. It is shown that a large portion of the properties for binomial ideals proved in [7] can be easily extended to the difference case. We also identify a class of normal binomial difference ideals which are in a one to one correspondence with Laurent difference binomial ideals. With the help of this correspondence, properties proved for Laurent binomial difference ideals can be extended to the non-Laurent case.

In Section 6, four equivalent definitions for difference toric varieties are given. A difference variety is called toric if it is the Cohn closure of the values of a set of Laurent difference monomials. It is proved that

Theorem 1.3 A difference variety $X$ is toric if and only if one of the following properties is valid.

(1) $X \cong \operatorname{Spec}^{\sigma}(\mathcal{F}\{M\})$, where $M$ is a finitely generated affine $\mathbb{N}[x]$-module in $\mathbb{Z}[x]^{m}$ and $\mathcal{F}\{M\}=\left\{\sum_{\boldsymbol{\alpha} \in M} a_{\boldsymbol{\alpha}} \mathbb{T}^{\boldsymbol{\alpha}} \mid a_{\boldsymbol{\alpha}} \in \mathcal{F}, a_{\boldsymbol{\alpha}} \neq 0\right.$ for finitely many $\left.\boldsymbol{\alpha}\right\}$ for a set of difference indeterminates $\mathbb{T}=\left\{t_{1}, \ldots, t_{m}\right\}$.

(2) The defining ideal of $X$ is a toric difference ideal, that is, $\mathbb{I}(X)=\left[\mathbb{Y}^{\mathbf{f}^{+}}-\mathbb{Y}^{\mathbf{f}^{-}} \mid \mathbf{f} \in L\right]$, where $L \subset \mathbb{Z}[x]^{n}$ is a $\mathbb{Z}[x]$-saturated $\mathbb{Z}[x]$-lattice and $\mathbf{f}^{+}, \mathbf{f}^{-}$are the positive and negative parts of $\mathbf{f}$, respectively. 
(3) $X$ contains a difference torus $T^{*}$ as a Cohn open subset and with a group action of $T^{*}$ on $X$ extending the natural group action of $T^{*}$ on itself.

In the algebraic case, any prime binomial ideal of the form $\left(\mathbb{Y}^{\mathbf{f}^{+}}-\mathbb{Y}^{\mathbf{f}^{-}} \mid \mathbf{f} \in L\right)$ has a monomial parametrization and hence is toric [7]. In the difference case, this is not valid (see Example 6.18). Also different from the algebraic case, the difference torus is not necessarily isomorphic to $\left(\mathbb{A}^{*}\right)^{n}$ (see Example 6.29), and this makes the definition of difference torus more complicated.

It is shown that the difference sparse resultant can be defined as the difference Chow form of a difference toric variety, and a Jacobi style order bound for a difference toric variety is derived from this connection.

In Section[7, algorithms are given to check whether a $\mathbb{Z}[x]$-lattice is $\mathbb{Z}-, x_{-}, \mathrm{M}-, \mathrm{P}-$, or $\mathbb{Z}[x]$ saturated, or equivalently, whether a Laurent binomial difference ideal is prime, reflexive, well-mixed, perfect, or toric. If the answer is negative, we can also compute the $\mathbb{Z}_{-}^{-}, x_{-}^{-}, \mathrm{M}_{-}^{-}$, $\mathrm{P}$-, or $\mathbb{Z}[x]$-saturation of $L$. Based on the above algorithms, we give algorithms to compute the reflexive, well-mixed, and perfect closures of a Laurent binomial difference ideal and an algorithm to decompose a perfect binomial difference ideal as the intersection of reflexive prime difference ideals. More precisely, we have

Theorem 1.4 Let $\mathcal{F}$ be an algebraically closed and inversive difference field and $F=$ $\left\{f_{1}, \ldots, f_{s}\right\} \subset \mathcal{F}\{\mathbb{Y}\}$ a set of difference binomials. Then we can compute difference regular and coherent ascending chains $\mathcal{A}_{1}, \ldots, \mathcal{A}_{t}$ such that

$$
\{F\}=\cap_{i=1}^{t} \operatorname{sat}\left(\mathcal{A}_{i}\right)
$$

where for each $i, \mathcal{A}_{i}$ consists of either $y_{c} \in \mathbb{Y}$ or difference binomials, and $\operatorname{sat}\left(\mathcal{A}_{i}\right)$ is a reflexive prime difference ideal. If $t=0$, we mean $\{F\}=[1]$.

The above result is stronger than the general decomposition algorithm given in [10] in that a finitely generated perfect difference ideal is decomposed as the intersection of reflexive prime difference ideals. For general difference polynomials, this is still an open problem, because we do not know how to check whether $\operatorname{sat}(\mathcal{A})$ is a reflexive prime difference ideal for a difference ascending chain $\mathcal{A}$.

Finally, we make a comparison with differential algebra. The study of binomial differential ideals is more difficult, because the differentiation of a binomial is generally not a binomial anymore. Differential varieties were defined in [23] and were used to connect the differential Chow form [9] and differential sparse resultant. But, contrary to the difference case, the defining ideal for a differential toric variety is generally not binomial and further study of differential toric varieties is not carried out.

\section{Preliminaries about difference algebra}

In this section, some basic notations and preliminary results about difference algebra and characteristic set for difference polynomial systems will be given. For more details about 
difference algebra, please refer to [3, 15, 20, 33]. For more details about characteristic set for difference polynomial systems, please refer to [10].

\subsection{Difference polynomial and Laurent difference polynomial}

An ordinary difference field, or simply a $\sigma$-field, is a field $\mathcal{F}$ with a third unitary operation $\sigma$ satisfying that for any $a, b \in \mathcal{F}, \sigma(a+b)=\sigma(a)+\sigma(b), \sigma(a b)=\sigma(a) \sigma(b)$, and $\sigma(a)=0$ if and only if $a=0$. We call $\sigma$ the transforming operator of $\mathcal{F}$. If $a \in \mathcal{F}, \sigma(a)$ is called the transform of $a$ and is denoted by $a^{(1)}$. And for $n \in \mathbb{Z}_{>0}, \sigma^{n}(a)=\sigma^{n-1}(\sigma(a))$ is called the $n$-th transform of $a$ and denoted by $a^{(n)}$, with the usual assumption $a^{(0)}=a$. If $\sigma^{-1}(a)$ is defined for each $a \in \mathcal{F}, \mathcal{F}$ is called inversive. Every difference field has an inversive closure 3. A typical example of inversive difference field is $\mathbb{Q}(\lambda)$ with $\sigma(f(\lambda))=f(\lambda+1)$.

In this paper, $\mathcal{F}$ is assumed to be inversive and of characteristic zero. Furthermore, we use $\sigma$ - as the abbreviation for difference or transformally.

We introduce the following useful notation. Let $x$ be an algebraic indeterminate and $p=\sum_{i=0}^{s} c_{i} x^{i} \in \mathbb{Z}[x]$. For $a$ in any $\sigma$-over field of $\mathcal{F}$, denote

$$
a^{p}=\prod_{i=0}^{s}\left(\sigma^{i} a\right)^{c_{i}} .
$$

For instance, $a^{x^{2}-1}=a^{(2)} / a$. It is easy to check that for $p, q \in \mathbb{Z}[x]$, we have

$$
a^{p+q}=a^{p} a^{q}, \quad a^{p q}=\left(a^{p}\right)^{q} .
$$

By $a^{[n]}$ we mean the set $\left\{a, a^{(1)}, \ldots, a^{(n)}\right\}$. If $S$ is a set of elements, we denote $S^{[n]}=\cup_{a \in S} a^{[n]}$.

Let $S$ be a subset of a $\sigma$-field $\mathcal{G}$ which contains $\mathcal{F}$. We will denote respectively by $\mathcal{F}[S]$, $\mathcal{F}(S), \mathcal{F}\{S\}$, and $\mathcal{F}\langle S\rangle$ the smallest subring, the smallest subfield, the smallest $\sigma$-subring, and the smallest $\sigma$-subfield of $\mathcal{G}$ containing $\mathcal{F}$ and $S$. If we denote $\Theta(S)=\left\{\sigma^{k} a \mid k \geq 0, a \in S\right\}$, then we have $\mathcal{F}\{S\}=\mathcal{F}[\Theta(S)]$ and $\mathcal{F}\langle S\rangle=\mathcal{F}(\Theta(S))$.

A subset $\mathcal{S}$ of a $\sigma$-extension field $\mathcal{G}$ of $\mathcal{F}$ is said to be $\sigma$-dependent over $\mathcal{F}$ if the set $\left\{\sigma^{k} a \mid a \in \mathcal{S}, k \geq 0\right\}$ is algebraically dependent over $\mathcal{F}$, and is said to be $\sigma$-independent over $\mathcal{F}$, or to be a family of $\sigma$-indeterminates over $\mathcal{F}$ in the contrary case. In the case $\mathcal{S}$ consists of one element $\alpha$, we say that $\alpha$ is $\sigma$-algebraic or $\sigma$-transcendental over $\mathcal{F}$, respectively. The maximal subset $\Omega$ of $\mathcal{G}$ which are $\sigma$-independent over $\mathcal{F}$ is said to be a $\sigma$-transcendence basis of $\mathcal{G}$ over $\mathcal{F}$. We use $\triangle \operatorname{tr}$.deg $\mathcal{G} / \mathcal{F}$ to denote the $\sigma$-transcendence degree of $\mathcal{G}$ over $\mathcal{F}$, which is the cardinal number of $\Omega$.

Now suppose $\mathbb{Y}=\left\{y_{1}, \ldots, y_{n}\right\}$ is a set of $\sigma$-indeterminates over $\mathcal{F}$. The elements of $\mathcal{F}\{\mathbb{Y}\}=\mathcal{F}\left[y_{j}^{(k)}: j=1, \ldots, n ; k \in \mathbb{N}\right]$ are called $\sigma$-polynomials over $\mathcal{F}$ in $\mathbb{Y}$, and $\mathcal{F}\{\mathbb{Y}\}$ itself is called the $\sigma$-polynomial ring over $\mathcal{F}$ in $\mathbb{Y}$. A $\sigma$-polynomial ideal, or simply a $\sigma$-ideal, $\mathcal{I}$ in $\mathcal{F}\{\mathbb{Y}\}$ is an ordinary algebraic ideal which is closed under transforming, i.e. $\sigma(\mathcal{I}) \subset \mathcal{I}$. If $\mathcal{I}$ also has the property that $a^{(1)} \in \mathcal{I}$ implies that $a \in \mathcal{I}$, it is called a reflexive $\sigma$-ideal. And a prime $\sigma$-ideal is a $\sigma$-ideal which is prime as an ordinary algebraic polynomial ideal. For convenience, a prime $\sigma$-ideal is assumed not to be the unit ideal in this paper. A $\sigma$-ideal $\mathcal{I}$ is called well-mixed if $f g \in \mathcal{I}$ implies $f g^{x} \in \mathcal{I}$ for $f, g \in \mathcal{F}\{\mathbb{Y}\}$. A $\sigma$-ideal $\mathcal{I}$ is called perfect 
if for any $a \in \mathbb{N}[x] \backslash\{0\}$ and $p \in \mathcal{F}\{\mathbb{Y}\}, p^{a} \in \mathcal{I}$ implies $p \in \mathcal{I}$. If $S$ is a subset of $\mathcal{F}\{\mathbb{Y}\}$, we use $(S),[S],\langle S\rangle$, and $\{S\}$ to denote the algebraic ideal, the $\sigma$-ideal, the well-mixed $\sigma$-ideal, and the perfect $\sigma$-ideal generated by $S$.

An $n$-tuple over $\mathcal{F}$ is an $n$-tuple of the form $\eta=\left(\eta_{1}, \ldots, \eta_{n}\right)$ where the $\eta_{i}$ are selected from a $\sigma$-overfield of $\mathcal{F}$. For a $\sigma$-polynomial $f \in \mathcal{F}\{\mathbb{Y}\}, \eta$ is called a $\sigma$-zero of $f$ if when substituting $y_{i}^{(j)}$ by $\eta_{i}^{(j)}$ in $f$, the result is 0 .

An $n$-tuple $\eta$ is called a generic zero of a $\sigma$-ideal $\mathcal{I} \subset \mathcal{F}\{\mathbb{Y}\}$ if for any $P \in \mathcal{F}\{\mathbb{Y}\}$ we have $P(\eta)=0 \Leftrightarrow P \in \mathcal{I}$. It is well known that a $\sigma$-ideal possesses a generic zero if and only if it is a reflexive prime $\sigma$-ideal other than the unit ideal [3, p.77]. Let $\mathcal{I}$ be a reflexive prime $\sigma$-ideal and $\eta$ a generic zero of $\mathcal{I}$. The dimension of $\mathcal{I}$ is defined to be $\triangle \operatorname{tr}$.deg $\mathcal{F}\langle\eta\rangle / \mathcal{F}$.

We now define the concept of $\sigma$-variety. Let $\mathcal{F}$ be an inversive $\sigma$-field, following [33, we denote the category of $\sigma$-field extensions of $\mathcal{F}$ by $\mathscr{E}_{\mathcal{F}}$, the category of $\mathcal{E}^{n}$ by $\mathscr{E}_{\mathcal{F}}$ where $\mathcal{E} \in \mathscr{E}_{\mathcal{F}}$. Let $(\mathbb{A})^{n}$ be the functor from $\mathscr{E}_{\mathcal{F}}$ to $\mathscr{E}_{\mathcal{F}}^{n}$ satisfying $(\mathbb{A})^{n}(\mathcal{E})=(\mathcal{E})^{n}$ where $\mathcal{E} \in \mathscr{E}_{\mathcal{F}}$. A $\sigma$-variety over $\mathcal{F}$ is a functor $\mathbb{V}$ from $\mathscr{E}_{\mathcal{F}}$ to the category of sets with the form $\mathbb{V}(P)$ for $P \subset \mathcal{F}\{\mathbb{Y}\}$ satisfying $\mathbb{V}_{\mathcal{E}}(P)=\left\{\eta \in \mathcal{E}^{n} \mid \forall p \in P, p(\eta)=0\right\}$. It is well known that $\sigma$-varieties are in a one to one correspondence with perfect $\sigma$-ideals.

For $\mathbf{f}=\left(f_{1}, \ldots, f_{n}\right)^{\tau} \in \mathbb{Z}[x]^{n}$, we define $\mathbb{Y}^{\mathbf{f}}=\prod_{i=1}^{n} y_{i}^{f_{i}} . \mathbb{Y}^{\mathbf{f}}$ is called a Laurent $\sigma$-monomial in $\mathbb{Y}$ and $\mathbf{f}$ is called its support. A vector $\mathbf{f}=\left(f_{1}, \ldots, f_{n}\right)^{\tau} \in \mathbb{Z}[x]^{n}$ is said to be normal if the leading coefficient of $f_{s}$ is positive, where $s$ is the largest subscript such that $f_{s} \neq 0$.

A Laurent $\sigma$-polynomial over $\mathcal{F}$ in $\mathbb{Y}$ is an $\mathcal{F}$-linear combinations of Laurent $\sigma$-monomials in $\mathbb{Y}$. Clearly, the set of all Laurent $\sigma$-polynomials form a commutative $\sigma$-ring under the obvious sum, product, and the usual transforming operator $\sigma$, where all Laurent $\sigma$-monomials are invertible. We denote the $\sigma$-ring of Laurent $\sigma$-polynomials with coefficients in $\mathcal{F}$ by $\mathcal{F}\left\{\mathbb{Y}^{ \pm}\right\}$.

Let $p$ be a Laurent $\sigma$-polynomial in $\mathcal{F}\left\{\mathbb{Y}^{ \pm}\right\}$. An $n$-tuple $\left(a_{1}, \ldots, a_{n}\right)$ over $\mathcal{F}$ with each $a_{i} \neq 0$ is called a nonzero $\sigma$-solution of $p$ if $p\left(a_{1}, \ldots, a_{n}\right)=0$. The concept of generic point for a Laurent $\sigma$-ideal can be defined similarly and it can be proved that a proper Laurent $\sigma$-ideal is reflexive and prime if and only if it has a generic point.

\subsection{Characteristic set for a difference polynomial system}

Let $f$ be a $\sigma$-polynomial in $\mathcal{F}\{\mathbb{Y}\}$. The order of $f$ w.r.t. $y_{i}$ is defined to be the greatest number $k$ such that $y_{i}^{(k)}$ appears effectively in $f$, denoted by ord $\left(f, y_{i}\right)$. If $y_{i}$ does not appear in $f$, then we set $\operatorname{ord}\left(f, y_{i}\right)=-\infty$. The order of $f$ is defined to be $\max _{i} \operatorname{ord}\left(f, y_{i}\right)$, that is, $\operatorname{ord}(f)=\max _{i} \operatorname{ord}\left(f, y_{i}\right)$.

The elimination ranking $\mathscr{R}$ on $\Theta(\mathbb{Y})=\left\{\sigma^{k} y_{i} \mid 1 \leq i \leq n, k \geq 0\right\}$ is used in this paper: $\sigma^{k} y_{i}>\sigma^{l} y_{j}$ if and only if $i>j$ or $i=j$ and $k>l$, which is a total order over $\Theta(\mathbb{Y})$. By convention, $1<\theta y_{j}$ for all $\theta y_{j} \in \Theta(\mathbb{Y})$.

Let $f$ be a $\sigma$-polynomial in $\mathcal{F}\{\mathbb{Y}\}$. The greatest $y_{j}^{(k)}$ w.r.t. $\mathscr{R}$ which appears effectively in $f$ is called the leader of $f$, denoted by $\operatorname{ld}(f)$ and correspondingly $y_{j}$ is called the leading variable of $f$, denoted by $\operatorname{lvar}(f)=y_{j}$. The leading coefficient of $f$ as a univariate polynomial 
in $\operatorname{ld}(f)$ is called the initial of $f$ and is denoted by $\mathrm{I}_{f}$.

Let $p$ and $q$ be two $\sigma$-polynomials in $\mathcal{F}\{\mathbb{Y}\}$. $q$ is said to be of higher rank than $p$ if

1) $\operatorname{ld}(q)>\operatorname{ld}(p)$, or

2) $\operatorname{ld}(q)=\operatorname{ld}(p)=y_{j}^{(k)}$ and $\operatorname{deg}\left(q, y_{j}^{(k)}\right)>\operatorname{deg}\left(p, y_{j}^{(k)}\right)$.

Suppose $\operatorname{ld}(p)=y_{j}^{(k)}$. $q$ is said to be reduced w.r.t. $p$ if $\operatorname{deg}\left(q, y_{j}^{(k+l)}\right)<\operatorname{deg}\left(p, y_{j}^{(k)}\right)$ for all $l \in \mathbb{N}$.

A finite sequence of nonzero $\sigma$-polynomials $\mathcal{A}=A_{1}, \ldots, A_{m}$ is said to be a difference ascending chain, or simply a $\sigma$-chain, if

1) $m=1$ and $A_{1} \neq 0$ or

2) $m>1, A_{j}>A_{i}$ and $A_{j}$ is reduced w.r.t. $A_{i}$ for $1 \leq i<j \leq m$.

A $\sigma$-chain $\mathcal{A}$ can be written as the following form

$$
\mathcal{A}=\left\{\begin{array}{l}
A_{11}, \ldots, A_{1 k_{1}} \\
\ldots \\
A_{p 1}, \ldots, A_{p k_{p}}
\end{array}\right.
$$

where $\operatorname{lvar}\left(A_{i j}\right)=y_{c_{i}}$ for $j=1, \ldots, k_{i}$ and $\operatorname{ord}\left(A_{i j}, y_{c_{i}}\right)<\operatorname{ord}\left(A_{i l}, y_{c_{i}}\right)$ for $j<l$.

Example 2.1 The following are three $\sigma$-chains

$$
\begin{array}{llll}
\mathcal{A}_{1}=y_{1}^{x}-1, & y_{1}^{2} y_{2}^{2}-1, & y_{2}^{x}-1 \\
\mathcal{A}_{2}=y_{1}^{2}-1, & y_{1}^{x}-y_{1}, & y_{2}^{2}-1, & y_{2}^{x}-y_{2} \\
\mathcal{A}_{3}=y_{2}^{2}-y_{1}^{x}, & y_{3}^{2}-y_{1}, & y_{3}^{x}-y_{2}
\end{array}
$$

Let $\mathcal{A}=A_{1}, A_{2}, \ldots, A_{t}$ be a $\sigma$-chain with $\mathrm{I}_{i}$ as the initial of $A_{i}$, and $f$ any $\sigma$-polynomial. Then there exists an algorithm, which reduces $f$ w.r.t. $\mathcal{A}$ to a polynomial $r$ that is reduced w.r.t. $\mathcal{A}$ and satisfies the relation

$$
\prod_{i=1}^{t} \mathrm{I}_{i}^{e_{i}} \cdot f \equiv r, \bmod [\mathcal{A}],
$$

where the $e_{i} \in \mathbb{N}[x]$. The $\sigma$-polynomial $r=\operatorname{prem}(f, \mathcal{A})$ is called the $\sigma$-remainder of $f$ w.r.t. $\mathcal{A}[10$.

A $\sigma$-chain $\mathcal{C}$ contained in a $\sigma$-polynomial set $\mathcal{S}$ is said to be a characteristic set of $\mathcal{S}$, if $\mathcal{S}$ does not contain any nonzero element reduced w.r.t. $\mathcal{C}$. A characteristic set $\mathcal{C}$ of a $\sigma$-ideal $\mathcal{J}$ reduces to zero all elements of $\mathcal{J}$.

Let $\mathcal{A}: A_{1}, \ldots, A_{t}$ be a $\sigma$-chain, $I_{i}=\mathrm{I}\left(A_{i}\right), y_{l_{i}}^{\left(o_{i}\right)}=\operatorname{ld}\left(A_{i}\right) . \mathcal{A}$ is called regular if for any $j \in \mathbb{N}, I_{i}^{x^{j}}$ is invertible w.r.t $\mathcal{A}[10]$ in the sense that $\left[A_{1}, \ldots, A_{i-1}, I_{i}^{x^{j}}\right]$ contains a nonzero $\sigma$-polynomial involving no $y_{l_{i}}^{\left(o_{i}+k\right)}, k=0,1, \ldots$ To introduce the concept of coherent $\sigma$ chain, we need to define the $\Delta$-polynomial first. If $A_{i}$ and $A_{j}$ have distinct leading variables, we define $\Delta\left(A_{i}, A_{j}\right)=0$. If $A_{i}$ and $A_{j}(i<j)$ have the same leading variable $y_{l}$, then $o_{i}=\operatorname{ord}\left(A_{i}, y_{l}\right)<o_{j}=\operatorname{ord}\left(A_{j}, y_{l}\right)$. Define

$$
\Delta\left(A_{i}, A_{j}\right)=\operatorname{prem}\left(\left(A_{i}\right)^{x^{o_{j}-o_{i}}}, A_{j}\right) .
$$


Then $\mathcal{A}$ is called coherent if $\operatorname{prem}\left(\Delta\left(A_{i}, A_{j}\right), \mathcal{A}\right)=0$ for all $i<j[10$.

Let $\mathcal{A}$ be a $\sigma$-chain. Denote $\mathbb{I}_{\mathcal{A}}$ to be the minimal multiplicative set containing the initials of elements of $\mathcal{A}$ and their transforms. The saturation ideal of $\mathcal{A}$ is defined to be

$$
\operatorname{sat}(\mathcal{A})=[\mathcal{A}]: \mathbb{I}_{\mathcal{A}}=\left\{p \in \mathcal{F}\{\mathbb{Y}\}: \exists h \in \mathbb{I}_{\mathcal{A}} \text {, s.t. } h p \in[A]\right\} .
$$

The following result is needed in this paper.

Theorem 2.2 [10, Theorem 3.3] $A \sigma$-chain $\mathcal{A}$ is a characteristic set of $\operatorname{sat}(A)$ if and only if $\mathcal{A}$ is regular and coherent.

\section{$3 \mathbb{Z}[x]$-lattice}

In this section, we prove basic properties of $\mathbb{Z}[x]$-lattices, which will play the role of lattices in the study of binomial ideals and toric varieties.

\subsection{Gröbner basis and generalized Hermite normal form}

For brevity, a $\mathbb{Z}[x]$-module in $\mathbb{Z}[x]^{n}$ is called a $\mathbb{Z}[x]$-lattice. Since $\mathbb{Z}[x]$ is a Noetherian ring, we have

Lemma 3.1 Any $\mathbb{Z}[x]$-lattice is finitely generated.

As a consequence, any $\mathbb{Z}[x]$-lattice $L$ has a finite set of generators $\mathbb{f}=\left\{\mathbf{f}_{1}, \ldots, \mathbf{f}_{s}\right\} \subset \mathbb{Z}[x]^{n}$ :

$$
L=\operatorname{Span}_{\mathbb{Z}[x]}\left\{\mathbf{f}_{1}, \ldots, \mathbf{f}_{s}\right\}=\left(\mathbf{f}_{1}, \ldots, \mathbf{f}_{s}\right) .
$$

A matrix representation of $\mathbf{f}$ or $L$ is

$$
M=\left[\mathbf{f}_{1}, \ldots, \mathbf{f}_{s}\right]_{n \times s},
$$

with $\mathbf{f}_{i}$ to be the $i$-th column of $M$. We also denote $L=(M)$.

Definition 3.2 The rank of a $\mathbb{Z}[x]$-lattice $L$ is defined to be the rank of any matrix representation of $L$.

The concept of rank is clearly well defined.

A standard form to represent the submodules in $\mathbb{Z}[x]^{n}$ is the Gröbner basis. We list some basic concepts and properties of Gröbner basis of modules. For details, please refer to [4].

Denote $\boldsymbol{\epsilon}_{i}$ to be the $i$-th standard basis vector $(0, \ldots, 0,1,0, \ldots, 0)^{\tau} \in \mathbb{Z}[x]^{n}$, where 1 lies in the $i$-th row of $\boldsymbol{\epsilon}_{i}$. A monomial $\mathbf{m}$ in $\mathbb{Z}[x]^{n}$ is an element of the form $a x^{k} \boldsymbol{\epsilon}_{i} \in \mathbb{Z}[x]^{n}$, where $a \in \mathbb{Z}$ and $k \in \mathbb{N}$. The following monomial order $>$ of $\mathbb{Z}[x]^{n}$ will be used in this paper: $a x^{\alpha} \boldsymbol{\epsilon}_{i}>b x^{\beta} \boldsymbol{\epsilon}_{j}$ if $i>j$, or $i=j$ and $\alpha>\beta$, or $i=j, \alpha=\beta$, and $|a|>|b|$.

With the above order, any $\mathbf{f} \in \mathbb{Z}[x]^{n}$ can be written in a unique way as a linear combination of monomials,

$$
\mathbf{f}=\sum_{i=1}^{s} \mathbf{f}_{i},
$$


where $\mathbf{f}_{i} \neq 0$ and $\mathbf{f}_{1}>\mathbf{f}_{2}>\cdots>\mathbf{f}_{s}$. The leading term of $\mathbf{f}$ is defined to be $\mathbf{L T}(\mathbf{f})=\mathbf{f}_{1}$. For any $\mathbb{G} \subset \mathbb{Z}[x]^{n}$, we denote by $\mathbf{L T}(\mathbb{G})$ the set of leading terms of $\mathbb{G}$.

The order $>$ can be extended to elements of $\mathbb{Z}[x]^{n}$ as follows: for $\mathbf{f}, \mathbf{g} \in \mathbb{Z}[x]^{n}, \mathbf{f}<\mathbf{g}$ if and only if $\mathbf{L T}(\mathbf{f})<\mathbf{L T}(\mathbf{g})$.

Let $\mathbb{G} \subset \mathbb{Z}[x]^{n}$ and $\mathbf{f} \in \mathbb{Z}[x]^{n}$. We say that $\mathbf{f}$ is $G$-reduced with respect to $\mathbb{G}$ if any monomial of $\mathbf{f}$ is not a multiple of $\mathbf{L T}(\mathbf{g})$ by an element in $\mathbb{Z}[x]$ for any $\mathbf{g} \in \mathbb{G}$.

Definition 3.3 A finite set $\mathbb{E}=\left\{\mathbf{f}_{1}, \ldots, \mathbf{f}_{s}\right\} \subset \mathbb{Z}[x]^{n}$ is called a Gröbner basis for the $\mathbb{Z}[x]$ lattice $L$ generated by $\mathbb{f}$ if $(\mathbf{L T}(L))=(\mathbf{L T}(\mathbb{H}))$. A Gröbner basis $\mathbb{f}$ is called reduced if for any $\mathbf{f} \in \mathbb{f}, \mathbf{f}$ is $G$-reduced with respect to $\mathbb{f} \backslash\{\mathbf{f}\}$.

Let $\mathbb{f}$ be a Gröbner basis. Then any $\mathbf{f} \in \mathbb{Z}[x]^{n}$ can be reduced to a unique normal form by $\mathbb{f}$, denoted by $\operatorname{grem}(\mathbf{f}, \mathbb{f})$, which is G-reduced with respect to $\mathbb{f}$.

Definition 3.4 Let $\mathbf{f}, \mathbf{g} \in \mathbb{Z}[x]^{n}, \mathbf{L T}(\mathbf{f})=a x^{k} \boldsymbol{\epsilon}_{i}, \mathbf{L T}(\mathbf{g})=b x^{s} \boldsymbol{\epsilon}_{j}$, and $t=\max (k, s)$. Then the S-vector of $\mathbf{f}$ and $\mathbf{g}$ is defined as follows: if $i \neq j$ then $S(\mathbf{f}, \mathbf{g})=0$; otherwise assume $|a| \geq|b|$ and let

$$
S(\mathbf{f}, \mathbf{g})=x^{t-k} \mathbf{f}-c x^{t-s} \mathbf{g}
$$

where $c=s\left\lfloor\frac{|a|}{|b|}\right\rfloor$ and $s$ is the sign of $a b$.

In the later case, let $a=b c+r$. Then $0 \leq|r|<|b|$ and $\mathbf{L T}(S(\mathbf{f}, \mathbf{g}))=r x^{t} \boldsymbol{\epsilon}_{i}$. Using the above notations, we have the following basic property for Gröbner basis [17.

Theorem 3.5 (Buchberger's Criterion) For a set $\mathbb{f}=\left\{\mathbf{f}_{1}, \ldots, \mathbf{f}_{s}\right\} \subset \mathbb{Z}[x]^{n}$, the following statements are equivalent.

1) $\mathbb{f}$ is a Gröbner basis.

2) $\operatorname{grem}\left(S\left(\mathbf{f}_{i}, \mathbf{f}_{j}\right), G\right)=0$ for all $i, j$.

3) $\mathbf{f} \in(\mathbb{f})$ if and only if $\operatorname{grem}(\mathbf{f}, \mathbb{f})=0$.

We will study the structure of a Gröbner basis for a $\mathbb{Z}[x]$-lattice by introducing the concept of generalized Hermite normal form. First, we consider the case of $n=1$.

Lemma 3.6 Let $B=\left\{b_{1}, \ldots, b_{k}\right\}$ be a reduced Gröbner basis of a $\mathbb{Z}[x]$-module in $\mathbb{Z}[x]$, $b_{1}<\cdots<b_{k}$, and $\mathbf{L T}\left(b_{i}\right)=c_{i} x^{d_{i}} \in \mathbb{N}[x]$. Then

1) $0 \leq d_{1}<d_{2}<\cdots<d_{k}$.

2) $c_{k}|\cdots| c_{2} \mid c_{1}$ and $c_{i} \neq c_{i+1}$ for $1 \leq i \leq k-1$.

3) $\frac{c_{i}}{c_{k}} \mid b_{i}$ for $1 \leq i<k$. Moreover if $\widetilde{b}_{1}$ is the primitive part of $b_{1}$, then $\widetilde{b}_{1} \mid b_{i}$ for $1<i \leq k$.

4) The S-polynomial $S\left(b_{i}, b_{j}\right)$ can be reduced to zero by $B$ for any $i, j$.

Proof: 1) and 4) are consequences of Theorem 3.5. To prove 2), assume that there exists an $l$ such that $c_{l-1}|\cdots| c_{2} \mid c_{1}$ but $c_{l} \backslash c_{l-1}$. Let $r=\operatorname{gcd}\left(c_{l}, c_{l-1}\right)=p_{1} c_{l}+p_{2} c_{l-1}$, where $p_{1}, p_{2} \in \mathbb{Z}$. Then $|r|<\left|c_{l-1}\right|$ and $|r|<\left|c_{l}\right|$. Since $c_{l-1}|\cdots| c_{2} \mid c_{1}$, we have $|r|<\left|c_{i}\right|, i=1, \ldots, l$. Let 
$g=p_{1} b_{l}+p_{2} x^{d_{l}-d_{l-1}} b_{l-1}$. Then $\mathbf{L T}(g)=r x^{d_{l}}$ which is reduced w.r.t. $B$ and $g \in(B)$, contradicting to the definition of Gröbner bases.

We prove 3) by induction on $k$. When $k=2$, let $b_{1}=c_{1} x^{d_{1}}+s_{11} x^{d_{1}-1}+\cdots+s_{1 d_{1}}$ and $b_{2}=c_{2} x^{d_{2}}+s_{21} x^{d_{2}-1}+\cdots+s_{2 d_{2}}$. Then, $c_{2} \mid c_{1}$ and $d_{1}<d_{2}$. Let $c_{1}=c_{2} t$, we need to show $t \mid b_{1}$. Since the S-polynomial $S\left(b_{1}, b_{2}\right)=t b_{2}-x^{d_{2}-d_{1}} b_{1}$ can be reduced to zero by $b_{1}$, we have $t b_{2}-x^{d_{2}-d_{1}} b_{1}=u(x) b_{1}$, where $u(x) \in \mathbb{Z}[x]$ and $\operatorname{deg}(u(x))<d_{2}-d_{1}$. Then, $t b_{2}=\left(x^{d_{2}-d_{1}}+u(x)\right) b_{1}$, and $t \mid b_{1}$ follows since $x^{d_{2}-d_{1}}+u(x)$ is a primitive polynomial in $\mathbb{Z}[x]$. The claim is true. Assume that for $k=l-1$, the claim is true, then $\widetilde{b}_{1} \mid b_{i}$ for $1 \leq i \leq l-1$. We will prove the claim for $k=l$. Since $S\left(b_{1}, b_{l}\right)=\frac{c_{1}}{c_{l}} b_{l}-x^{d_{l}-d_{1}} b_{1}$ can be reduced to zero by $B$. We have $\frac{c_{1}}{c_{l}} b_{l}-x^{d_{l}-d_{1}} b_{1}=\sum_{i=1}^{l-1} f_{i} b_{i}$ with $f_{i} \in \mathbb{Z}[x]$ and $\operatorname{deg}\left(f_{i} b_{i}\right) \leq d_{l}-1$. Then, $\frac{c_{1}}{c_{l}} b_{l}=x^{d_{l}-d_{1}} b_{1}+\sum_{i=1}^{l-1} f_{i} b_{i}$. By induction, $\widetilde{b}_{1}$ is a factor of the right hand side of the above equation. Thus $\widetilde{b}_{1} \mid b_{l}$. Let $b_{i}=s_{i} b_{1}^{\prime}$ for $1 \leq i \leq l$, we have $\frac{c_{1}}{c_{l}} s_{l}=x^{d_{l}-d_{1}} s_{1}+\sum_{i=1}^{l-1} f_{i} s_{i}$ where $\operatorname{deg}\left(s_{i}\right)=d_{i}-d_{1}$ and $s_{1} \in \mathbb{Z}$. Since $\operatorname{deg}\left(f_{i} s_{i}\right) \leq d_{l}-d_{1}-1$, we have $\frac{c_{1}}{c_{l}} \mid s_{1}$ and $\frac{c_{1}}{c_{l}} \mid b_{1}$. For any $1 \leq i<j<l$, assume $\frac{c_{i}}{c_{l}} \mid b_{i}$. We will show that $\frac{c_{j}}{c_{l}} \mid b_{j}$. Since $S\left(b_{j-1}, b_{j}\right)=\frac{c_{j-1}}{c_{j}} b_{j}-x^{d_{j}-d_{j-1}} b_{j-1}=\sum_{i=1}^{j-1} f_{i} b_{i}$, we have $\frac{c_{j-1}}{c_{l}}$ is a factor of the right hand side of the above equation, for $c_{j-1}\left|c_{j-2}\right| \cdots \mid c_{1}$. Then, $\frac{c_{j-1}}{c_{l}} \mid \frac{c_{j-1}}{c_{j}} b_{j}$ and $\frac{c_{j}}{c_{l}} \mid b_{j}$. The claim is proved.

Example 3.7 Here are three Gröbner bases in $\mathbb{Z}[x]:\{2, x\},\left\{12,6 x+6,3 x^{2}+3 x, x^{3}+x^{2}\right\}$, $\left\{9 x+3,3 x^{2}+4 x+1\right\}$.

To give the structure of a reduced Gröbner basis similar to that in Example 3.7 we introduce the concept of generalized Hermite normal form. Let

$$
\mathcal{C}=\left[\begin{array}{lllllllllll}
c_{1,1} & \ldots & c_{1, l_{1}} & c_{1, l_{1}+1} & \ldots & \ldots & \ldots & \ldots & \ldots & \ldots \\
\ldots & \ldots & \ldots & \ldots & \ldots & \ldots & \ldots & \ldots & \ldots & \ldots \\
c_{r_{1}, 1} & \ldots & c_{r_{1}, l_{1}} & c_{r_{1}, l_{1}+1} & \ldots & \ldots & \ldots & \ldots & \ldots & \ldots \\
0 & \ldots & 0 & c_{r_{1}+1,1} & \ldots & c_{r_{1}+1, l_{2}} & \ldots & \ldots & \ldots & \ldots & \ldots \\
\ldots & \ldots & \ldots & \ldots & \ldots & \ldots & \ldots & \ldots & \ldots & \ldots & \ldots \\
0 & \ldots & 0 & c_{r_{2}, 1} & \ldots & c_{r_{2}, l_{2}} & \ldots & \ldots & \ldots & \ldots & \ldots \\
\ldots & \ldots & \ldots & \ldots & \ldots & \ldots & \ldots & \ldots & \ldots & \ldots & \ldots \\
0 & \ldots & 0 & 0 & \ldots & 0 & \ldots & 0 & c_{r_{t-1}+1,1} & \ldots & c_{r_{t-1}+1, l_{t}} \\
\ldots & \ldots & \ldots & \ldots & \ldots & \ldots & \ldots & \ldots & \ldots & \ldots & \ldots \\
0 & \ldots & 0 & 0 & \ldots & 0 & \ldots & 0 & c_{r_{t}, 1} & \ldots & c_{r_{t}, l_{t}}
\end{array}\right]_{m \times s}
$$

whose elements are in $\mathbb{Z}[x]$. It is clear that $m=r_{t}$ and $s=\sum_{i=1}^{t} l_{i}$. We denote by $\mathbf{c}_{k}=\mathbf{c}_{r_{i}, j}$ to be the $k$-th column of the matrix $\mathcal{C}$, where $k=l_{1}+l_{2}+\ldots+l_{i-1}+j, 1 \leq j \leq l_{i}$. Assume

$$
c_{i, j}=c_{i, j, 0} x^{d_{i j}}+\cdots+c_{i, j, d_{i j}} .
$$

Then the leading monomial of $\mathbf{c}_{r_{i}, j}$ is $c_{r_{i}, j, 0} x^{d_{r_{i}, j}} \boldsymbol{\epsilon}_{r_{i}}$.

Definition 3.8 The matrix $\mathcal{C}$ in (4) is called a generalized Hermite normal form if it satisfies the following conditions:

1) $0 \leq d_{r_{i}, 1}<d_{r_{i}, 2}<\cdots<d_{r_{i}, l_{i}}$ for any $i$. 
2) $c_{r_{i}, l_{i}, 0}|\cdots| c_{r_{i}, 2,0} \mid c_{r_{i}, 1,0}$.

3) $S\left(\mathbf{c}_{r_{i}, j_{1}}, \mathbf{c}_{r_{i}, j_{2}}\right)=x^{d_{r_{i}, j_{2}}-d_{r_{i}, j_{1}}} \mathbf{c}_{r_{i}, j_{1}}-\frac{c_{r_{i}, j_{1}, 0}}{c_{r_{i}, j_{2}, 0}} \mathbf{c}_{r_{i}, j_{2}}$ can be reduced to zero by the column vectors of the matrix for any $1 \leq i \leq t, 1 \leq j_{1}<j_{2} \leq l_{i}$.

4) $\mathbf{c}_{r_{i}, j}$ is G-reduced w.r.t. the column vectors of the matrix other than $\mathbf{c}_{r_{i}, j}$, for any $1 \leq$ $i \leq t, 1 \leq j \leq l_{i}$.

It is clear that $\left\{c_{r_{i}, 1}, \ldots, c_{r_{i}, l_{i}}\right\}$ is a reduced Gröbner basis in $\mathbb{Z}[x]$. Then, as a consequence of Theorem 3.5 and Lemma 3.6, we have

Theorem $3.9 \mathbb{f}=\left\{\mathbf{f}_{1}, \ldots, \mathbf{f}_{s}\right\} \subset \mathbb{Z}[x]^{n}$ is a reduced Gröbner basis such that $\mathbf{f}_{1}<\mathbf{f}_{2}<\cdots<$ $\mathbf{f}_{s}$ if and only if the matrix representation for $\mathbb{f}$ is a generalized Hermite normal form.

The following property of $\mathcal{C}$ will be used later.

Lemma 3.10 Let $\mathcal{C}$ be given in (4) be a generalized Hermite normal form and $L=(\mathcal{C})$. Then $\operatorname{rk}(L)=t$ and $L$ is a free $\mathbb{Z}[x]$-module if $l_{i}=1$ for $i=1, \ldots, t$.

Example 3.11 The following matrices are generalized Hermite normal forms

$$
M_{1}=\left[\begin{array}{lll}
x & 2 & 0 \\
0 & 2 & x
\end{array}\right], M_{2}=\left[\begin{array}{llll}
2 & x-1 & 0 & 0 \\
0 & 0 & 2 & x-1
\end{array}\right], M_{3}=\left[\begin{array}{lll}
-x & -1 & 0 \\
2 & 0 & -1 \\
0 & 2 & x
\end{array}\right]
$$

whose columns constitute the reduced Gröbner bases of the $\mathbb{Z}[x]$-lattices. For instance, $\mathbb{f}_{1}=$ $\left\{[x, 0]^{\tau},[2,2]^{\tau},[0, x]^{\tau}\right\}$ is the reduced Gröbner basis of $\left(\mathbb{F}_{1}\right)$.

Example 3.12 The number of generators for a $\mathbb{Z}[x]$-lattice depends on how to arrange the row elements. For instance, if we move the third row of $M_{3}$ in Example 3.11 to be the first row, then we have $\widetilde{M}_{3}=\left[\begin{array}{lll}0 & 2 & x \\ -x & -1 & 0 \\ 2 & 0 & -1\end{array}\right]$. Then $\left(M_{3}\right)$ has another set of generators $(2,-1,0)^{T}$ and $(x, 0,-1)^{T}$. In other words, if $N_{3}=\left[\begin{array}{ll}2 & x \\ -1 & 0 \\ 0 & -1\end{array}\right]$, then $\left(\widetilde{M}_{3}\right)=\left(N_{3}\right)$ which is a free $\mathbb{Z}[x]$-lattice.

Let $\mathcal{C}$ be defined in (44) and $k \in \mathbb{N}$. Introduce the following notations:

$$
\begin{aligned}
\mathcal{C}_{-} & =\cup_{i=1}^{t} \cup_{k=1}^{l_{i}-1}\left\{\mathbf{c}_{r_{i}, k}, x \mathbf{c}_{r_{i}, k}, \ldots, x^{\operatorname{deg}\left(c_{r_{i}, k+1}\right)-\operatorname{deg}\left(c_{r_{t}, k}\right)-1} \mathbf{c}_{r_{i}, k}\right\} \\
\mathcal{C}^{+} & =\cup_{i=1}^{t} \cup_{k=0}^{\infty}\left\{x^{k} \mathbf{c}_{r_{i}, l_{i}}\right\} . \\
\mathcal{C}_{\infty} & =\mathcal{C}_{-} \cup \mathcal{C}^{+}
\end{aligned}
$$

$\mathcal{C}_{\infty}$ is called the extension of $\mathcal{C}$. 
Example 3.13 Let $\mathcal{C}=\left[\begin{array}{lllll}6 & 3 x & 0 & 3 & 2 x \\ 0 & 0 & 6 & 3 x & x^{3}+x\end{array}\right]$. Then $\mathcal{C}_{-}=\left[\begin{array}{llll}6 & 0 & 3 & 3 x \\ 0 & 6 & 3 x & 3 x^{2}\end{array}\right]$ and

$$
\mathcal{C}_{\infty}=\left[\begin{array}{lllllllllll}
6 & 3 x & 3 x^{2} & 3 x^{3} & \cdots & 0 & 3 & 3 x & 2 x & 2 x^{2} & \cdots \\
0 & 0 & 0 & 0 & \cdots & 6 & 3 x & 3 x^{2} & x^{3}+x & x^{4}+x^{2} & \cdots
\end{array}\right]
$$

We need the following properties about the extension of $\mathcal{C}$. By saying the infinite set $\mathcal{C}_{\infty}$ is linear dependent over $\mathbb{Z}$, we mean any finite subset of $\mathcal{C}_{\infty}$ is linear dependent over $\mathbb{Z}$. Otherwise, $\mathcal{C}_{\infty}$ is said to be linear independent.

Lemma 3.14 Let $\mathcal{C}$ be a generalized Hermite normal form. The columns of $\mathcal{C}_{\infty}$ are linear independent over $\mathbb{Z}$.

Proof: Suppose $\mathcal{C}$ is given in (44). The leading term of $\mathbf{c} \in \mathcal{C}_{\infty}$ is $\mathbf{L T}(\mathbf{c})=a x^{l} \boldsymbol{\epsilon}_{r_{i}}$ for $i=1, \ldots, t$ and $l \in \mathbb{N}$. Furthermore, for two different $\mathbf{c}_{1}$ and $\mathbf{c}_{2}$ in $\mathcal{C}_{S}$ such that $\mathbf{L T}\left(\mathbf{c}_{1}\right)=a x^{l_{1}} \boldsymbol{\epsilon}_{r_{i}}$ and $\mathbf{L T}\left(\mathbf{c}_{2}\right)=b x^{l_{2}} \boldsymbol{\epsilon}_{r_{i}}$, we have $l_{1} \neq l_{2}$. Then $\mathbf{L T}\left(\mathcal{C}_{\infty}\right)=\left\{a_{i l_{i}} x^{l_{i}} \boldsymbol{\epsilon}_{r_{i}} \mid i=1, \ldots, t ; l_{i}=\right.$ $\left.d_{i 1}, d_{i 1}+1, \ldots ; a_{i l_{i}} \in \mathbb{Z}\right\}$ are linear independent over $\mathbb{Z}$, where $d_{i 1}$ is from (5). Then $\mathcal{C}_{\infty}$ are also linear independent over $\mathbb{Z}$.

Lemma 3.15 Let $\mathcal{C}$ be a generalized Hermite normal form. Then any $\mathbf{g} \in(\mathcal{C})$ can be written uniquely as a linear combination of finitely many elements of $\mathcal{C}_{\infty}$ over $\mathbb{Z}$.

Proof: $\mathrm{g} \in(\mathcal{C})$ can be written as a linear combination of elements of $\mathcal{C}_{\infty}$ over $\mathbb{Z}$ by the procedure to compute grem $(\mathrm{g}, \mathcal{C})=0$ [4]. The uniqueness is a consequence of Lemma 3.14, $\square$

Note that syzygies among elements of $\mathcal{C}$ are not linear combinations over $\mathbb{Z}$. For instance, let $\mathbf{f}_{1}=[2,0]^{\tau}$ and $\mathbf{f}_{2}=[x-1,0]^{\tau}$. Then we have $(x-1) \mathbf{f}_{1}-2 \mathbf{f}_{2}=\mathbf{0}$ which is not a linear representation of $\mathbf{0}$ over $\mathbb{Z}$.

\subsection{Kernel of a matrix in $\mathbb{Z}[x]^{n \times s}$}

Let $F=\left[\mathbf{f}_{1}, \ldots, \mathbf{f}_{s}\right]_{n \times s} \in \mathbb{Z}[x]^{n \times s}$, where $\mathbf{f}_{i} \in \mathbb{Z}[x]^{n}$. The kernel of $F$ is

$$
\operatorname{ker}(F)=\left\{X \in \mathbb{Z}[x]^{s} \mid F X=\mathbf{0}\right\} .
$$

It is clear that $\operatorname{ker}(F)$ is a $\mathbb{Z}[x]$-lattice in $\mathbb{Z}[x]^{s}$, which has the following important property.

Lemma 3.16 Let $F=\left[\mathbf{f}_{1}, \ldots, \mathbf{f}_{s}\right]_{n \times s} \in \mathbb{Z}[x]^{n \times s}$. Then $\operatorname{ker}(F)$ is a free $\mathbb{Z}[x]$-module.

Proof: Without loss of generality, we assume $\mathbb{f}=\left\{\mathbf{f}_{1}, \ldots, \mathbf{f}_{s}\right\}$ is a the generalized Hermite normal form which means $\mathbb{f}$ is a Gröbner basis. Let $S\left(\mathbf{f}_{i}, \mathbf{f}_{j}\right)=m_{i j} \mathbf{f}_{i}-m_{j i} \mathbf{f}_{j}$ and $\operatorname{grem}\left(S\left(\mathbf{f}_{i}, \mathbf{f}_{j}\right), \mathbb{f}\right)=\sum_{k} c_{k} \mathbf{f}_{k}$ be the normal representation of in terms of the Gröbner basis $\mathbb{f}$. Then the syzygy polynomial $\widetilde{S}\left(\mathbf{f}_{i}, \mathbf{f}_{j}\right)$

$$
\widetilde{S}\left(\mathbf{f}_{i}, \mathbf{f}_{j}\right)=m_{i j} \boldsymbol{\epsilon}_{i}-m_{j i} \boldsymbol{\epsilon}_{j}-\sum_{k} c_{k} \boldsymbol{\epsilon}_{k}
$$


is an element in $\mathbb{Z}[x]^{s}$, where $\boldsymbol{\epsilon}_{k}$ is the $k$-th standard basis vector of $\mathbb{Z}[x]^{s}$. Define an order in $\mathbb{Z}[x]^{s}$ as follows: $a x^{\alpha} \boldsymbol{\epsilon}_{i} \prec b x^{\beta} \boldsymbol{\epsilon}_{j}$ if $\mathbf{L T}\left(a x^{\alpha} \mathbf{f}_{i}\right)>\mathbf{L T}\left(b x^{\beta} \mathbf{f}_{j}\right)$ in $\mathbb{Z}[x]^{n}$. We will use Schreyer's Theorem on page 224 of [4], which says that if $\left\{\mathbf{f}_{1}, \ldots, \mathbf{f}_{s}\right\}$ is a reduce Gröbner basis, then the syzygy polynomials $\widetilde{S}=\left\{\widetilde{S}\left(\mathbf{f}_{i}, \mathbf{f}_{j}\right)\right\}$ constitute a Gröbner basis of $\operatorname{ker}(M) \subset \mathbb{Z}[x]^{s}$ under the newly defined order $\prec$. By the proof of Schreyer's Theorem, the order $\prec$ is a monomial order. Rewrite $\mathbf{f}_{i}$ as the form (4), that is, $\left\{\mathbf{f}_{1}, \ldots, \mathbf{f}_{s}\right\}=\left\{\mathbf{c}_{r_{i}, j} \mid 1 \leq i \leq t, 1 \leq j \leq l_{i}\right\}$. Let $\mathbf{s}_{i j k}=\widetilde{S}\left(\mathbf{c}_{r_{i}, j}, \mathbf{c}_{r_{i}, k}\right)$ with $j<k$. By (2) of Definition 3.8, $\mathbf{L} \mathbf{T}_{\succ}\left(s_{i j k}\right)=\frac{c_{r_{i}, j, 0}}{c_{r_{i}, k, 0}} \boldsymbol{\epsilon}_{\omega_{i k}}$ where $\omega_{i k}=l_{1}+l_{2}+\cdots+l_{i-1}+k$ and $\frac{c_{r_{i}, j, 0}}{c_{r_{i}, k, 0}} \in \mathbb{Z}$.

We claim that

$$
\mathrm{H}=\left\{\mathbf{s}_{i, k-1, k}, 1 \leq i \leq t, 2 \leq k \leq l_{i}\right\}
$$

is a reduced Gröbner basis for $\operatorname{ker}(M)$. By Schreyer's Theorem, the set of $\mathbf{s}_{i j k}$ forms a Gröbner basis for $\operatorname{ker}(M)$. The claim will be proved if we can show that for $j=1, \ldots, k-2$, $\mathbf{L} \mathbf{T}_{\succ}\left(\mathbf{s}_{i j k}\right)=h_{i j k} \mathbf{L} \mathbf{T}_{\succ}\left(\mathbf{s}_{i k-1 k}\right)$, where $h_{i j k} \in \mathbb{Z}$. We have $\mathbf{L} \mathbf{T}_{\succ}\left(s_{i j k}\right)=\frac{c_{r_{i}, j, 0}}{c_{r_{i}, k, 0}} \boldsymbol{\epsilon}_{\omega_{i k}}, \mathbf{L} \mathbf{T}_{\succ}\left(s_{i k-1 k}\right)$ $=\frac{c_{r_{i}, k-1,0}}{c_{r_{i}, k, 0}} \boldsymbol{\epsilon}_{\omega_{i k}}$. Since $\frac{c_{r_{i}, j, 0}}{c_{r_{i}, k, 0}}=\frac{c_{r_{i}, j, 0}}{c_{r_{i}, k-1,0}} \frac{c_{r_{i}, k-1,0}}{c_{r_{i}, k, 0}}$, by (2) of Definition 3.8, $\frac{c_{r_{i}, j, 0}}{c_{r_{i}, k-1,0}}$ and $\frac{c_{r_{i}, k-1,0}}{c_{r_{i}, k, 0}}$ are in $\mathbb{Z}$. The claim is proved.

By Lemma 3.10 and the claim, the $\mathbb{Z}[x]$-lattice $(\mathrm{H})$ is free. By Proposition 3.10 of $[4$ (page $229)$, since $(\mathrm{H})$ is free and $\mathrm{H}$ is set of generators of $\operatorname{ker}(M), \operatorname{ker}(M)$ is also free.

Corollary 3.17 If $F=\left[\mathbf{f}_{1}, \ldots, \mathbf{f}_{s}\right]_{n \times s} \in \mathbb{Z}[x]^{n \times s}$ is a generalized Hermite normal form, then (17) is a reduced Gröbner basis of $\operatorname{ker}(F)$ under the order $\prec$ and $\operatorname{rk}(\operatorname{ker}(F))=s-\operatorname{rk}(F)$.

Proof: It suffices to show $\operatorname{rk}(\operatorname{ker}(F))=s-\operatorname{rk}(F)$. Suppose $F$ has the form (44). Then $\operatorname{rk}(F)=t . \quad \mathrm{H}$ in (7) is the reduced Gröber basis for $\operatorname{ker}(F)$. Note that the number of elements in $\mathrm{H}$ is $s-t$ and these elements are linearly independent since each of them has the different standard basis vector. Then $\operatorname{rk}(\operatorname{ker}(F))=s-t=s-\operatorname{rk}(F)$.

Example 3.18 In Example [3.11, $\mathbf{f}_{1}=[x, 0]^{\tau}, \mathbf{f}_{2}=[2,2]^{\tau}, \mathbf{f}_{3}=[0, x]^{\tau}$. Then $S\left(f_{2}, f_{3}\right)=$ $x \mathbf{f}_{2}-2 \mathbf{f}_{3}=[2 x, 0]^{\tau}=2 \mathbf{f}_{1}$ and the syzygy polynomial is $\mathbf{s}_{212}=x \boldsymbol{\epsilon}_{2}-2 \boldsymbol{\epsilon}_{3}-2 \boldsymbol{\epsilon}_{1}$. That is, $\operatorname{ker}(M)$ is generated by $[-2, x,-2]^{\tau}$.

Remark 3.19 $\operatorname{ker}(F)$ is the syzygy module of $M$, which can be computed by the Gröbner basis method in Chapter 6 of [4] or Proposition 3.8 in [4, p. 227].

\section{Laurent binomial $\sigma$-ideal}

In this section, we will prove some basic facts about Laurent binomial $\sigma$-ideals. As a tool, the characteristic set method for Laurent binomial $\sigma$-ideals will be presented.

\subsection{Laurent binomial $\sigma$-ideal}

In this section, several basic properties of binomial $\sigma$-ideals will be proved. 
By a Laurent $\sigma$-binomial in $\mathbb{Y}$, we mean a $\sigma$-polynomial with at most two terms, that is, $a \mathbb{Y}^{\mathbf{g}}+b \mathbb{Y}^{\mathbf{h}}$ where $a, b \in \mathcal{F}$ and $\mathbf{g}, \mathbf{h} \in \mathbb{Z}[x]^{n}$. A Laurent $\sigma$-binomial of the following form is said to be in normal form

$$
p=\mathbb{Y}^{\mathbf{f}}-c_{\mathbf{f}}
$$

where $c_{\mathbf{f}} \in \mathcal{F}^{*}=\mathcal{F} \backslash\{0\}$ and $\mathbf{f} \in \mathbb{Z}[x]^{n}$ is normal. The vector $\mathbf{f}$ is called the support of $p$. For $p=\mathbb{Y}^{\mathbf{f}}-c_{\mathbf{f}}$, we denote $\widehat{p}=-c_{\mathbf{f}}^{-1} \mathbb{Y}^{-\mathbf{f}} p=\mathbb{Y}^{-\mathbf{f}}-c_{\mathbf{f}}^{-1}$ which is called the inverse of $p$. Note that if $p$ is in a Laurent binomial $\sigma$-ideal $\mathcal{I}$, then $\widehat{p}$ is also in $\mathcal{I}$. It is clear that any Laurent $\sigma$-binomial $f$ which is not a unit can be written uniquely as

$$
f=a M\left(\mathbb{Y}^{\mathbf{f}}-c_{\mathbf{f}}\right)
$$

where $a \in \mathcal{F}^{*}, M$ is a Laurent $\sigma$-monomial, and $\mathbb{Y}^{\mathbf{f}}-c_{\mathbf{f}}$ is in normal form. Since $a M$ is a unit in $\mathcal{F}\left\{\mathbb{Y}^{ \pm}\right\}$, we can use the normal $\sigma$-binomial $\mathbb{Y}^{\mathbf{f}}-c_{\mathbf{f}}$ to represent $f$, and when we say a Laurent $\sigma$-binomial we always use its normal representation.

A Laurent $\sigma$-ideal is called binomial if it is generated by Laurent $\sigma$-binomials.

Lemma 4.1 Let $\mathbb{Y}^{\mathbf{f}_{i}}-c_{i}, i=1, \ldots, s$ be contained in a Laurent binomial $\sigma$-ideal $\mathcal{I}$ and $\mathbf{f}=a_{1} \mathbf{f}_{1}+\cdots+a_{s} \mathbf{f}_{s}$, where $a_{i} \in \mathbb{Z}[x]$. Then $\mathbb{Y}^{\mathbf{f}}-\prod_{i=1}^{s} c_{i}^{a_{i}}$ is in $\mathcal{I}$.

Proof: It is suffices to show that if $p_{1}=\mathbb{Y}^{\mathbf{f}_{1}}-c_{1} \in \mathcal{I}$ and $p_{2}=\mathbb{Y}^{\mathbf{f}_{2}}-c_{2} \in \mathcal{I}$, then $\mathbb{Y}^{n \mathbf{f}_{1}}-c_{1}^{n} \in \mathcal{I}$ for $n \in \mathbb{N}, \mathbb{Y}^{-\mathbf{f}_{1}}-c_{1}^{-1} \in \mathcal{I}, \mathbb{Y}^{x \mathbf{f}_{1}}-\sigma\left(c_{1}\right) \in \mathcal{I}$, and $\mathbb{Y}^{\mathbf{f}_{1}+\mathbf{f}_{2}}-c_{1} c_{2} \in \mathcal{I}$, which are indeed true since $\mathbb{Y}^{n \mathbf{f}_{1}}-c_{1}^{n}=\left(\mathbb{Y}^{\mathbf{f}_{1}}\right)^{n}-c_{1}^{n}$ contains $p_{1}$ as a factor, $\mathbb{Y}^{-\mathbf{f}_{1}}-c_{1}^{-1}=-c_{1}^{-1} \mathbb{Y}^{-\mathbf{f}_{1}}\left(\mathbb{Y}^{\mathbf{f}_{1}}-c_{1}\right) \in \mathcal{I}$, $\mathbb{Y}^{x \mathbf{f}_{1}}-\sigma\left(c_{1}\right)=\sigma\left(\mathbb{Y}^{\mathbf{f}_{1}}-c_{1}\right) \in \mathcal{I}$, and $\mathbb{Y}^{\mathbf{f}_{1}+\mathbf{f}_{2}}-c_{1} c_{2}=\mathbb{Y}^{\mathbf{f}_{1}}\left(\mathbb{Y}^{\mathbf{f}_{2}}-c_{2}\right)+c_{2}\left(\mathbb{Y}^{\mathbf{f}_{1}}-c_{1}\right) \in \mathcal{I}$.

As a direct consequence, we have

Corollary 4.2 Let $\mathcal{I}$ be a Laurent binomial $\sigma$-ideal and

$$
\mathbb{L}(\mathcal{I}):=\left\{\mathbf{f} \in \mathbb{Z}[x]^{n} \mid \exists c_{\mathbf{f}} \in \mathcal{F}^{*} \text { s.t. } \mathbb{Y}^{\mathbf{f}}-c_{\mathbf{f}} \in \mathcal{I}\right\} .
$$

Then $\mathbb{L}(\mathcal{I})$ is a $\mathbb{Z}[x]$-lattice, which is called the support lattice of $\mathcal{I}$, and a matrix representation for $\mathbb{L}(\mathcal{I})$ is called a matrix representation for $\mathcal{I}$.

The following lemma shows that a new set of generators of the support lattice of a Laurent binomial $\sigma$-ideal leads to a new set of generators for the $\sigma$-ideal.

Lemma 4.3 Let $\mathcal{I}=\left[\mathbb{Y}^{\mathbf{f}_{1}}-c_{1}, \ldots, \mathbb{Y}^{\mathbf{f}_{s}}-c_{s}\right]$ be a proper Laurent binomial $\sigma$-ideal and let $\mathbf{h}_{1}, \ldots, \mathbf{h}_{r}$ be another set of generators for the $\mathbb{Z}[x]$-lattice $\left(\mathbf{f}_{1}, \ldots, \mathbf{f}_{s}\right)$, and there exist $a_{i, k} \in$ $\mathbb{Z}[x]$ such that

$$
\mathbf{h}_{i}=\sum_{k=1}^{s} a_{i, k} \mathbf{f}_{k}, i=1, \ldots, r .
$$

Then $\mathcal{I}=\left[\mathbb{Y}^{\mathbf{h}_{1}}-\prod_{i=1}^{s} c_{i}^{a_{1, i}}, \ldots, \mathbb{Y}^{\mathbf{h}_{r}}-\prod_{i=1}^{s} c_{i}^{a_{r, i}}\right]$.

Proof: Let $f_{t}=\mathbb{Y}^{\mathbf{f}_{t}}-c_{t}, t=1, \ldots, s, g_{l}=\mathbb{Y}^{\mathbf{h}_{l}}-\prod_{i=1}^{s} c_{i}^{a_{l, i}}, l=1, \ldots, r$, and $\mathcal{I}_{1}=\left[g_{1}, \ldots, g_{r}\right]$. From Lemma 4.1, $\mathcal{I}_{1} \subset \mathcal{I}$. We now prove $\mathcal{I} \subset \mathcal{I}_{1}$. Since $\left(\mathbf{h}_{1}, \ldots, \mathbf{h}_{r}\right)=\left(\mathbf{f}_{1}, \ldots, \mathbf{f}_{s}\right)$, there 
exist $b_{i, k} \in \mathbb{Z}[x]$ such that $\mathbf{f}_{i}=\sum_{k=1}^{r} b_{i, k} \mathbf{h}_{k}$. Then, $\mathbb{Y}^{\mathbf{f}_{i}}=\mathbb{Y}^{\sum_{k=1}^{r} b_{i, k} \mathbf{h}_{k}}=\prod_{k=1}^{r}\left(\mathbb{Y}^{\mathbf{h}_{k}}\right)^{b_{i, k}}$. Replacing $\mathbb{Y}^{\mathbf{h}_{k}}$ by $g_{k}+\prod_{i=1}^{s} c_{i}^{a_{l, i}}$, we have $\mathbb{Y}^{\mathbf{f}_{i}}=\prod_{k=1}^{s}\left(g_{k}+\prod_{j=1}^{s} c_{j}^{a_{k, j}}\right)^{b_{i, k}}=\widetilde{g}+\widetilde{c}$, where $\widetilde{c}=\prod_{j=1}^{s} c_{j}^{\sum_{k=1}^{s} b_{i, k} a_{k, j}} \in \mathcal{F}$ and $\widetilde{g} \in \mathcal{I}_{1} \subset \mathcal{I}$. As a consequence, $\mathbb{Y}^{\mathbf{f}_{i}}-\widetilde{c}=\widetilde{g} \in \mathcal{I}$. Then $f_{i}-\left(\mathbb{Y}^{\mathbf{f}_{i}}-\widetilde{c}\right)=\widetilde{c}-c_{i} \in \mathcal{I}$. Since $\mathcal{I}$ is proper, we have $\widetilde{c}=c_{i}$ and hence $f_{i}=\mathbb{Y}^{\mathbf{f}_{i}}-\widetilde{\mathcal{c}} \in \mathcal{I}_{1}$. The lemma is proved.

The following lemma can be used to check whether a Laurent binomial $\sigma$-ideal is proper.

Lemma 4.4 Let $\mathcal{I}=\left[\mathbb{Y}^{\mathbf{f}_{1}}-c_{1}, \ldots, \mathbb{Y}^{\mathbf{f}_{s}}-c_{s}\right]$ be a Laurent binomial $\sigma$-ideal and let $M$ be the $n \times s$ matrix with columns $\mathbf{f}_{1}, \ldots, \mathbf{f}_{s}$. Furthermore, let $\operatorname{ker}(M)$ be generated by $\mathbf{u}_{1}, \ldots, \mathbf{u}_{t}$, where $\mathbf{u}_{i}=\left(u_{i, 1}, \ldots, u_{i s}\right)^{\tau} \in \mathbb{Z}[x]^{s}$. Then $\mathcal{I} \neq[1]$ if and only if $\prod_{i=1}^{s} c_{i}^{u_{l, i}}=1$ for $l=1, \ldots, t$.

Proof: " $\Rightarrow$ " Let $f_{i}=\mathbb{Y}^{\mathbf{f}_{i}}-c_{i}$. Suppose $c=\prod_{i=1}^{s} c_{i}^{u_{l, i}} \neq 1$ for some $l$. Replacing $c_{i}$ by $\mathbb{Y}^{\mathbf{f}_{i}}-f_{i}$ in the above equation and noting that $\mathbf{u}_{l} \in \operatorname{ker}(M)$, we have $c=\prod_{i=1}^{s} c_{i}^{u_{l, i}}=$ $\prod_{i=1}^{s}\left(y^{\mathbf{f}_{i}}-f_{i}\right)^{u_{l, i}}=\prod_{i=1}^{s} \mathbb{Y}^{M \cdot \mathbf{u}_{l}}+g=1+g$ where $g \in \mathcal{I}$. Then $0 \neq c-1 \in \mathcal{I}$ and $\mathcal{I}=[1]$, a contradiction.

"Æ" Suppose the contrary. Then there exist $g_{i} \in \mathcal{F}\left\{\mathbb{Y}^{ \pm}\right\}$such that

$$
g_{1} f_{1}+\cdots+g_{s} f_{s}=1 \text {. }
$$

Let $l$ be the maximal $c$ such that $y_{c}^{(k)}$ occurs in some $f_{i}$, o the largest $j$ such that $y_{l}^{(j)}$ occurs in some $f_{k}$, and $d=\max _{k=1}^{s} \operatorname{deg}\left(f_{k}, y_{l}^{(o)}\right)$. Let $f_{k}=\mathbb{Y}^{\mathbf{f}_{k}}-c_{k}=I_{k} y_{l}^{d x^{o}}-c_{k}$. Since (9) is an identity about the algebraic variables $y_{i}^{x^{j}}$, we can set $y_{l}^{d x^{o}}=c_{k} / I_{k}$ in (9) to obtain a new identity. In the new identity, $f_{k}$ becomes zero and the left hand side of (9) has at most $s-1$ summands. We will show that this procedure can be continued for the new identity. Then the left hand side of (9) will eventually becomes zero, and a contradiction is obtained and the lemma is proved.

If $\operatorname{ord}\left(f_{i}, y_{l}\right)<o$ or $\operatorname{ord}\left(f_{i}, y_{l}\right)=o$ and $\operatorname{deg}\left(f_{i}, y_{l}^{x^{o}}\right)<d$ for some $i$, then $f_{i}$ is not changed in the above procedure. Let us assume that for some $v, \operatorname{deg}\left(f_{v}, y_{l}^{x^{o}}\right)=d$ and $f_{v}=\mathbb{Y}^{\mathbf{f}_{v}}-c_{v}=I_{v} y_{l}^{d x^{o}}-c_{v}$. Then after the substitution, $f_{v}=c_{k} I_{v} / I_{k}-c_{v}=c_{k} \widetilde{f}_{v}$ where $\widetilde{f}_{v}=I_{v} / I_{k}-c_{v} / c_{k}$. We claim that either $\widetilde{f}_{v}=0$ or $I_{v} / I_{k}$ is a proper monomial, and as a consequence, the above substitution can continue. To prove the claim, it suffices to show that if $I_{v}=I_{k}$ then $c_{v}=c_{k}$. If $I_{v}=I_{k}$, then $\mathbf{f}_{v}=\mathbf{f}_{k}$, that is $\mathbf{f}_{v}-\mathbf{f}_{k}=0$ is a syzygy among $\mathbf{f}_{i}$ and let $\boldsymbol{\epsilon}_{v k}$ be the corresponding syzygy vector. Then $\boldsymbol{\epsilon}_{v k} \in \operatorname{ker}(M)$ can be written as a linear combination of $\mathbf{u}_{1}, \ldots, \mathbf{u}_{s}$. Let $\mathbf{c}=\left(c_{1}, \ldots, c_{s}\right)^{\tau}$. Then $c_{v} c_{k}^{-1}=\mathbf{c}^{\boldsymbol{\epsilon}_{v k}}$ can be written as a product of $\mathbf{c}^{\mathbf{u}_{l}}=\prod_{i=1}^{s} c_{i}^{u_{l, i}}=1$, and thus $c_{v} c_{k}^{-1}=1$.

\subsection{Characteristic set of Laurent binomial $\sigma$-ideal}

We show that the characteristic set method presented in section 2.2 can be modified to the case of Laurent binomial $\sigma$-ideals. First, assume that all Laurent $\sigma$-binomials are in normal form, which makes the concepts of order, leading variables, etc. unique. 
Second, when defining the concepts of rank and $q$ to be reduced w.r.t. $p$, we need to replace $\operatorname{deg}\left(p, y_{j}^{(o)}\right)$ by $\left|\operatorname{deg}\left(p, y_{j}^{(o)}\right)\right|$. Precisely, $q$ is said to be reduced w.r.t. $p$ if $\left|\operatorname{deg}\left(q, y_{j}^{(k+l)}\right)\right|<$ $\left|\operatorname{deg}\left(p, y_{j}^{(k)}\right)\right|$ for all $l \in \mathbb{N}$, where $\operatorname{ld}(p)=y_{j}^{(k)}$. For instance, $y_{1}^{-2 x} y_{2}-1$ is not reduced w.r.t. $y_{1}^{2}-1$. With these changes, the concepts of $\sigma$-chain and characteristic set can be defined in the Laurent $\sigma$-binomial case. For instance, the $\sigma$-chains in Example 2.1 become the following Laurent normal form:

$$
\begin{aligned}
& \mathcal{A}_{1}=y_{1}^{x}-1, \quad y_{1}^{2} y_{2}^{2}-1, \quad y_{2}^{x}-1 \\
& \mathcal{A}_{2}=y_{1}^{2}-1, \quad y_{1}^{-1} y_{1}^{x}-1, \quad y_{2}^{2}-1, \quad y_{2}^{-1} y_{2}^{x}-1 \\
& \mathcal{A}_{3}=y_{1}^{-x} y_{2}^{2}-1, \quad y_{1}^{-1} y_{3}^{2}-1, \quad y_{2}^{-1} y_{3}^{x}-1
\end{aligned}
$$

Third, the $\sigma$-remainder for two Laurent $\sigma$-binomials need to be modified as follows. We first consider how to compute $\operatorname{prem}(f, g)$ in the simple case: $o=\operatorname{ord}\left(f, y_{l}\right)=\operatorname{ord}\left(g, y_{l}\right)$, where $y_{l}=\operatorname{lvar}(g)$. Let $g=I_{g}\left(y_{l}^{(o)}\right)^{d}-c_{g}$, where $d=\operatorname{deg}\left(g, y_{l}^{(o)}\right)$ and $I_{g}$ is the initial of $g$. As mentioned above, $g$ is in normal form, that is $d>0$. Let $d_{f}=\operatorname{deg}\left(f, y_{l}^{(o)}\right)$ and $f=I_{f}\left(y_{l}^{(o)}\right)^{d_{f}}-c_{f}$. We consider two cases.

In the first case, let us assume $d_{f} \geq 0$. If $d_{f}<d_{g}$, then set $r=\operatorname{prem}_{1}(f, g)$ to be $f$. Otherwise, perform the following basic step

$$
r:=\operatorname{prem}_{1}(f, g)=\left(f-g \frac{I_{f}}{I_{g}}\left(y_{l}^{(o)}\right)^{d_{f}-d_{g}}\right) / c_{g}=\frac{I_{f}}{I_{g}}\left(y_{l}^{(o)}\right)^{d_{f}-d_{g}}-\frac{c_{f}}{c_{g}} .
$$

Let $\mathbf{h}_{r}, \mathbf{h}_{f}, \mathbf{f}_{g}$ be the supports of $r, f, g$, respectively. Then

$$
\mathbf{h}_{r}=\mathbf{h}_{f}-\mathbf{h}_{g} .
$$

Set $f=r$ and repeat the procedure $\operatorname{prem}_{1}$ for $f$ and $g$. Since $d_{f}$ decreases strictly after each iteration, the procedure will end and return $r$ which satisfies

$$
\begin{aligned}
r & =\frac{f}{c_{g}^{k}}-h g=\frac{I_{f}}{I_{g}^{k}}\left(y_{l}^{(o)}\right)^{d_{f}-k d_{g}}-\frac{c_{f}}{c_{g}^{k}} \\
\mathbf{h}_{r} & =\mathbf{h}_{f}-k \mathbf{h}_{g}
\end{aligned}
$$

where $k=\left\lfloor\frac{d_{f}}{d_{g}}\right\rfloor$ and $h \in \mathcal{F}\left\{\mathbb{Y}^{ \pm}\right\}$. Let prem $(f, g)=r$ or the inverse of $r$ in the case that $r$ is not in normal form.

In the second case, we assume $d_{f}<0$. The $\sigma$-remainder can be computed similar to the first case. Instead of $g$, we consider $\widehat{g}=\left(I_{g}\right)^{-1}\left(y_{l}^{(o)}\right)^{-d_{g}}-c_{g}^{-1}$. If $\left|d_{f}\right|<d_{g}$, then set $r=\operatorname{prem}_{1}(f, g)$ to be $f$. Otherwise, perform the following basic step

$$
r:=\operatorname{prem}_{1}(f, g)=c_{g}\left(f-\widehat{g} I_{g} I_{f}\left(y_{l}^{(o)}\right)^{d_{f}+d_{g}}\right)=I_{f} I_{g}\left(y_{l_{g}}^{(o)}\right)^{d_{f}+d_{g}}-c_{f} c_{g} .
$$

In this case, equation (11) becomes $\mathbf{h}_{r}=\mathbf{h}_{f}+\mathbf{h}_{g}$. To compute prem $(f, g)$, repeat the above basic step for $f=r$ until $\left|d_{f}\right|<d_{g}$.

For two general $\sigma$-binomials $f$ and $g, \operatorname{prem}(f, g)$ is defined as follows: if $f$ is reduced w.r.t $g$, set $\operatorname{prem}(f, g)=f$. Otherwise, let $y_{l}=\operatorname{lvar}(g), o_{f}=\operatorname{ord}\left(f, y_{l}\right)$, and $o_{g}=\operatorname{ord}\left(g, y_{l}\right)$. Define

$$
\operatorname{prem}(f, g)=\operatorname{prem}\left(\ldots, \operatorname{prem}\left(\operatorname{prem}\left(f, g^{\left(o_{f}-o_{g}\right)}\right), g^{\left(o_{f}-o_{g}-1\right)}\right), \ldots, g\right) .
$$


Let $\mathcal{A}: A_{1}, \ldots, A_{s}$ be a Laurent binomial $\sigma$-chain and $f$ a $\sigma$-binomial. Then define

$$
\operatorname{prem}(f, \mathcal{A})=\operatorname{prem}\left(\ldots, \operatorname{prem}\left(\operatorname{prem}\left(f, A_{s}\right), A_{s-1}\right), \ldots, A_{1}\right) .
$$

In summary, we have

Lemma 4.5 Let $\mathcal{A}=A_{1}, \ldots, A_{s}$ be a Laurent binomial $\sigma$-chain, $f$ a $\sigma$-binomial, and $r=$ $\operatorname{prem}(f, \mathcal{A})$. Then $r$ is reduced w.r.t. $\mathcal{A}$ and satisfies

$$
c f \equiv r, \bmod [\mathcal{A}] \text {, }
$$

where $c \in \mathcal{F}^{*}$. Furthermore, let the supports of $r$ and $f$ be $\mathbf{h}_{r}$ and $\mathbf{h}_{f}$, respectively. Then $\mathbf{h}_{f}-\mathbf{h}_{r}$ is in the $\mathbb{Z}[x]$-lattice generated by the supports of $A_{i}$.

Now, the concepts of Laurent binomial regular and coherent $\sigma$-chains and the characteristic set for Laurent binomial $\sigma$-ideals can be defined and Theorem 2.2 can be extended to the Laurent binomial case.

Theorem 4.6 A Laurent binomial $\sigma$-chain $\mathcal{A}$ is a characteristic set of $\operatorname{sat}(A)$ if and only if $\mathcal{A}$ is regular and coherent.

In the rest of this section, we will establish a relation between Gröbner bases of $\mathbb{Z}[x]$ lattices and characteristic sets of Laurent binomial $\sigma$-ideals. Let $\mathbb{f}=\left\{\mathbf{f}_{1}, \ldots, \mathbf{f}_{s}\right\}$ be a reduced Gröbner basis of a $\mathbb{Z}[x]$-lattice such that $\mathbf{f}_{1}<\mathbf{f}_{2}<\cdots<\mathbf{f}_{s}$.

Lemma 4.7 If $\mathbb{f}=\left\{\mathbf{f}_{1}, \ldots, \mathbf{f}_{s}\right\}$ is a reduced Gröbner basis of a $\mathbb{Z}[x]$-lattice, then $\mathbb{Y}^{\mathbf{f}_{1}}$ $c_{1}, \ldots, \mathbb{Y}^{\mathbf{f}_{s}}-c_{s}$ is a $\sigma$-chain for any $c_{i} \in \mathcal{F}^{*}$.

Proof: Let $A_{i}=\mathbb{Y}^{\mathbf{f}_{i}}-c_{i}$. Then for $i<j, A_{j}$ is of higher rank than $A_{i}$. $A_{j}$ is reduced w.r.t. $A_{i}$ if and only if $\mathbf{f}_{j}$ is G-reduced w.r.t. $\mathbf{f}_{i}$, which is valid due to Definition 3.8 .

Note that for a Laurent binomial $\sigma$-chain $\mathcal{A}$, we have $\operatorname{sat}(\mathcal{A})=[\mathcal{A}]$.

Lemma 4.8 Let $\mathbb{f}=\left\{\mathbf{f}_{1}, \ldots, \mathbf{f}_{s}\right\}$ be a reduced Gröbner basis of a $\mathbb{Z}[x]$-lattice and $\mathcal{A}$ the $\sigma$-chain $\mathbb{Y}^{\mathbf{f}_{1}}-c_{1}, \ldots, \mathbb{Y}^{\mathbf{f}_{s}}-c_{s}$. If $\mathcal{I}=[\mathcal{A}]$ is a proper Laurent binomial $\sigma$-ideal, then for $f=\mathbb{Y}^{\mathbf{f}}-c_{\mathbf{f}} \in \mathcal{I}, \operatorname{grem}(\mathbf{f}, \mathbb{f})=0$ if and only if $\operatorname{prem}(f, \mathcal{A})=0$.

Proof: Let us first consider prem ${ }_{1}$ in (10) for $f$ and $A_{i}=\mathbb{Y}^{\mathbf{f}_{i}}-c_{i}=I_{i}\left(y_{l_{i}}^{\left(o_{i}\right)}\right)^{d_{i}}-c_{i}$, where $\operatorname{ld}\left(A_{i}\right)=y_{l_{i}}$ and $I_{i}$ is the initial of $A_{i}$. From (11), the support of $r=\operatorname{prem}_{1}\left(f, A_{i}\right)$ is $\mathbf{f}-\mathbf{f}_{i}$.

It is clear that $\mathbf{L T}\left(\mathbf{f}_{i}\right)=d_{i} x^{o_{i}} \boldsymbol{\epsilon}_{l_{i}}$. Let $\mathbf{f}_{i}=d_{i} x^{O_{i}} \boldsymbol{\epsilon}_{l_{i}}+\overline{\mathbf{f}}_{i}$. Similarly, write $\mathbf{f}=d_{f} x^{o_{i}} \boldsymbol{\epsilon}_{l_{i}}+\bar{m}$ where $d_{f} x^{o_{i}} \boldsymbol{\epsilon}_{l_{i}}$ is the leading term of $\mathbf{f}$ w.r.t. $\boldsymbol{\epsilon}_{l_{i}}$ and $d_{f} \geq d_{i} \geq 0$. Then a basic step to compute $\operatorname{grem}\left(\mathbf{f}, \mathbf{f}_{i}\right)$ is to compute $\operatorname{grem}_{1}\left(\mathbf{f}, \mathbf{f}_{i}\right)=\mathbf{f}-\mathbf{f}_{i}=\left(d_{f}-d_{i}\right) x^{o_{i}} \boldsymbol{\epsilon}_{l_{i}}+\overline{\mathbf{f}}-\overline{\mathbf{f}}_{i}$, which is the support of $\operatorname{prem}_{1}\left(f, A_{i}\right)$.

As a consequence, using the basic step $\operatorname{grem}_{1}$ to compute grem $(\mathbf{f}, \mathbb{f})$, we have a sequence of elements in $\mathbb{Z}[x]^{n}: \mathbf{g}_{0}=\mathbf{f}, \mathbf{g}_{1}, \ldots, \mathbf{g}_{t}=\operatorname{grem}(\mathbf{f}, \mathbb{f})$. Correspondingly, using the basic step $\operatorname{prem}_{1}$ to compute $\operatorname{prem}(\mathbf{f}, \mathcal{A})$, we have a sequence of $\sigma$-binomials $f_{0}=f, f_{1}, \ldots, f_{t}=$ $\operatorname{prem}(f, \mathcal{A})$ such that the support of $f_{i}$ is $\mathbf{g}_{i}$. Let $f_{t}=\mathbb{Y}^{\mathbf{g}_{t}}-\widetilde{c}_{t}$. Since $\mathcal{I}$ is proper, $f_{t}=$ $\operatorname{prem}(f, \mathcal{A})=\mathbb{Y}^{\mathbf{g}_{t}}-\widetilde{c}_{t}=0$ if and only if $\mathbf{g}_{t}=\operatorname{grem}(\mathbf{f}, \mathbb{f})=0$. 
Lemma 4.9 Let $\mathbb{f}=\left\{\mathbf{f}_{1}, \ldots, \mathbf{f}_{s}\right\}$ be a reduced Gröbner basis of a $\mathbb{Z}[x]$-lattice and $\mathcal{A}$ the $\sigma$-chain $\mathbb{Y}^{\mathbf{f}_{1}}-c_{1}, \ldots, \mathbb{Y}^{\mathbf{f}_{s}}-c_{s}$. If $\mathcal{I}=[\mathcal{A}]$ is a proper Laurent binomial $\sigma$-ideal, then $\mathcal{A}$ is a regular and coherent $\sigma$-chain.

Proof: By Lemma 4.7, $\mathcal{A}$ is a $\sigma$-chain. Since the initials of $\mathcal{A}$ are $\sigma$-monomials which are units in $\mathcal{F}\left\{\mathbb{Y}^{ \pm}\right\}, \mathcal{A}$ is regular. We still need to prove that $\mathcal{A}$ is coherent.

Let $A_{i}=\mathbb{Y}^{\mathbf{f}_{i}}-c_{i}$ and $A_{j}=\mathbb{Y}^{\mathbf{f}_{j}}-c_{j}(i<j)$ have the same leading variable $y_{l}$, and $A_{i}=I_{i} y_{l}^{d_{i} x^{o_{i}}}-c_{i}, A_{j}=I_{j} y_{l}^{d_{j} x^{o_{j}}}-c_{j}$. From Definition 3.8, we have $o_{i}<o_{j}$ and $d_{j} \mid d_{i}$. Let $d_{i}=t d_{j}$ where $t \in \mathbb{N}$. According to (12), we have

$$
\Delta\left(A_{i}, A_{j}\right)=\operatorname{prem}\left(\left(A_{i}\right)^{x^{o_{j}-o_{i}}}, A_{j}\right)=\frac{\left(I_{i}\right)^{x^{o_{j}-o_{i}}}}{I_{j}^{t}}-\frac{\left(c_{i}\right)^{x^{o_{j}-o_{i}}}}{c_{j}^{t}} .
$$

Then the support of $\Delta\left(A_{i}, A_{j}\right)$ is $x^{o_{j}-o_{i}} \mathbf{f}_{i}-\frac{d_{i}}{d_{j}} \mathbf{f}_{j}$.

Since $\mathbf{L T}\left(A_{i}\right)=d_{i} x^{o_{i}} \boldsymbol{\epsilon}_{l}$ and $\mathbf{L T}\left(A_{j}\right)=d_{j} x^{o_{j}} \boldsymbol{\epsilon}_{l}$, we have $N=\operatorname{lcm}\left(d_{i} x^{o_{i}}, d_{j} x^{o_{j}}\right)=d_{i} x^{o_{j}}$. According to Definition 3.4 the S-vector of $\mathbf{f}_{i}$ and $\mathbf{f}_{j}$ is

$$
S\left(\mathbf{f}_{i}, \mathbf{f}_{j}\right)=x^{o_{j}-o_{i}} \mathbf{f}_{i}-\frac{d_{i}}{d_{j}} \mathbf{f}_{j} .
$$

Since $\mathbb{f}$ is a Gröbner basis, we have $\operatorname{grem}\left(S\left(\mathbf{f}_{i}, \mathbf{f}_{j}\right), \mathbb{f}\right)=0$. Also note that the support of $\Delta\left(A_{i}, A_{j}\right)$ is $S\left(\mathbf{f}_{i}, \mathbf{f}_{j}\right)$. Then by Lemma 4.8, $\operatorname{prem}\left(\Delta\left(A_{i}, A_{j}\right), \mathcal{A}\right)=0$, that is, $\mathcal{A}$ is coherent.

We summarize the results in this section as the following theorems.

Theorem 4.10 Let $\mathbb{f}=\left\{\mathbf{f}_{1}, \ldots, \mathbf{f}_{s}\right\}$ be a reduced Gröbner basis of a $\mathbb{Z}[x]$-lattice and $\mathcal{A}$ the $\sigma$-chain $\mathbb{Y}^{\mathbf{f}_{1}}-c_{1}, \ldots, \mathbb{Y}^{\mathbf{f}_{s}}-c_{s}$. If $\mathcal{I}=[\mathcal{A}]$ is a proper Laurent binomial $\sigma$-ideal, then $\mathcal{A}$ is a characteristic set of $\mathcal{I}$.

Proof: This is a direct consequence of Lemma 4.9 and Theorem 4.6 .

In the following theorem, we show how to compute the characteristic set for a Laurent binomial $\sigma$-ideal using Gröbner bases of $\mathbb{Z}[x]$-lattices.

Theorem 4.11 Let $\mathcal{I}=\left[\mathbb{Y}^{\mathbf{f}_{1}}-c_{1}, \ldots, \mathbb{Y}^{\mathbf{f}_{s}}-c_{s}\right]$ be a proper Laurent binomial $\sigma$-ideal, $\mathbb{h}=$ $\left[\mathbf{h}_{1}, \ldots, \mathbf{h}_{r}\right]$ the reduced Gröbner basis of the $\mathbb{Z}[x]$-lattice $\left(\mathbf{f}_{1}, \ldots, \mathbf{f}_{s}\right)$, and $\mathbf{h}_{i}=\sum_{k=1}^{s} a_{i, k} \mathbf{f}_{k}$ for $a_{i, k} \in \mathbb{Z}[x]$. Let $\mathcal{A}$ be $\mathbb{Y}^{\mathbf{h}_{1}}-\prod_{i=1}^{s} c_{i}^{a_{1, i}}, \ldots, \mathbb{Y}^{\mathbf{h}_{r}}-\prod_{i=1}^{s} c_{i}^{a_{r, i}}$. Then $\mathcal{I}=[\mathcal{A}]$ and $\mathcal{A}$ is a characteristic set of $\mathcal{I}$.

Proof: By Lemma 4.3, $\mathcal{I}=[\mathcal{A}]$. By Theorem 4.10, $\mathcal{A}$ is a characteristic set of $\mathcal{I}$.

Corollary 4.12 Let $\mathcal{I}$ be a Laurent reflexive prime binomial $\sigma$-ideal in $\mathcal{F}\left\{\mathbb{Y}^{ \pm}\right\}$. Then $\operatorname{dim}(\mathcal{I})=n-\operatorname{rk}(\mathbb{L}(\mathcal{I}))$.

Proof: Suppose $\mathcal{C}=\left[\mathbf{c}_{1}, \ldots, \mathbf{c}_{s}\right]$ in (44) is the matrix representation for $\mathcal{I}$. Since $\mathcal{I}$ is reflexive and prime, $\mathcal{I}$ has a characteristic set of form $\mathcal{A}: \mathbb{Y}^{\mathbf{c}_{1}}-c_{1}, \ldots \mathbb{Y}^{\mathbf{c}_{s}}-c_{s}$. By Theorem 4.3 of [10], $\operatorname{dim}(\mathcal{I})=n-t=n-\operatorname{rk}(\mathbb{L}(\mathcal{I}))$. 
Corollary 4.13 A Laurent binomial $\sigma$-ideal is radical.

Proof: By Theorem 4.11, $\mathcal{I}=\left[\mathbb{Y}^{\mathbf{h}_{1}}-c_{1}, \ldots, \mathbb{Y} \mathbf{h}-c_{r}\right]$, where $\mathcal{A}: \mathbb{Y}^{\mathbf{h}_{1}}-c_{1}, \ldots, \mathbb{Y}^{\mathbf{h}_{r}}-c_{r}$ is the characteristic set of $\mathcal{I}$. Let $A_{i}=\mathbb{Y}^{\mathbf{h}_{i}}-c_{i}$ and $y_{l_{i}}^{\left(o_{i}\right)}=\operatorname{ld}\left(A_{i}\right) . \mathcal{A}$ is also saturated in the sense that its separant $\frac{\partial A_{i}}{\partial y_{l_{i}}^{\left(o_{i}\right)}}$ are $\sigma$-monomials and hence units in $\mathcal{F}\left\{\mathbb{Y}^{ \pm}\right\}$. Then similar to the differential case [1], it can be shown that $\operatorname{sat}(\mathcal{A})=[\mathcal{A}]$ is a radical $\sigma$-ideal.|

Finally, we show that the converse of Lemma 4.9 is also true.

Theorem 4.14 Let $\mathbf{f}_{i} \in \mathbb{Z}[x]^{n}$ and $c_{i} \in \mathcal{F}^{*}$ for $i=1, \ldots, s$. $\mathcal{A}: \mathbb{Y}^{\mathbf{f}_{1}}-c_{1}, \ldots, \mathbb{Y}^{\mathbf{f}_{s}}-c_{s}$ is a regular and coherent $\sigma$-chain if and only if $\mathbb{f}=\left\{\mathbf{f}_{1}, \ldots, \mathbf{f}_{s}\right\}$ is a reduced Gröbner basis and $[\mathcal{A}]$ is a proper Laurent binomial $\sigma$-ideal. In this case, the support lattice of $[\mathcal{A}]$ is $(\mathbb{\mathbb { H }})$.

Proof: Lemma 4.9 proves one side of the theorem. For the other direction, let $\mathcal{A}$ be a regular and coherent $\sigma$-chain. From the proof of Lemma 4.7, $\mathbf{f}_{i}$ is G-reduced to $\mathbf{f}_{i}$ for $i \neq j$. By Theorem 4.6, $\mathcal{A}$ is a characteristic set of $\operatorname{sat}(\mathcal{A})=[\mathcal{A}]$, which means $[\mathcal{A}]$ is proper. Use the notations introduced in the proof of Lemma 4.9. Since $S\left(\mathbf{f}_{i}, \mathbf{f}_{j}\right)$ is the support of $\Delta\left(A_{i}, A_{j}\right)$, by the proof of Lemma 4.8, $\mathbf{f}_{i j}=\operatorname{grem}\left(S\left(\mathbf{f}_{i}, \mathbf{f}_{j}\right), \mathbb{f}\right)$ is the support of $\operatorname{prem}\left(\Delta\left(A_{i}, A_{j}\right), \mathcal{A}\right)$. Since $\mathcal{A}$ is coherent, $\operatorname{prem}\left(\Delta\left(A_{i}, A_{j}\right), \mathcal{A}\right)=\mathbb{Y}^{\mathbf{f}_{i j}}-c=0$ for any $i$ and $j$, which implies $\mathbf{f}_{i j}=\operatorname{grem}\left(S\left(\mathbf{f}_{i}, \mathbf{f}_{j}\right), \mathbb{f}\right)=0$ and hence $\mathbb{f}$ is a reduced Gröbner basis.

It remains to show that the support lattice of $[\mathcal{A}]$ is $(\mathbb{E})$. By Theorem 4.10 and Lemma 4.8. $f=\mathbb{Y}^{\mathrm{g}}-c \in[\mathcal{A}]$ if and only if $\operatorname{prem}(f, \mathcal{A})=0$, which is equivalent to $\operatorname{grem}(\mathrm{g}, \mathbb{f})=0$, that is $\mathbb{L}([\mathcal{A}])=(\mathbb{H})$.

\subsection{Partial character and Laurent binomial $\sigma$-ideal}

In this section, we will show that proper Laurent binomial $\sigma$-ideals can be described uniquely with their partial characters.

Definition 4.15 A partial character $\rho$ on $\mathbb{Z}[x]^{n}$ is a homomorphism from a $\mathbb{Z}[x]$-lattice $L_{\rho}$ to the multiplicative group $\mathcal{F}^{*}$ satisfying $\rho(x \mathbf{f})=\sigma(\rho(\mathbf{f}))$ for $\mathbf{f} \in L_{\rho}$.

Partial characters can be defined on any $\mathbb{Z}[x]$-lattice $L$. A trivial partial character on $L$ is defined by setting $\rho(\mathbf{f})=1$ for any $\mathbf{f} \in L$.

Let $\rho$ be a partial character over $\mathbb{Z}[x]^{n}$ and $L_{\rho}=\left(\mathbf{f}_{1}, \ldots, \mathbf{f}_{s}\right)$, where $\mathbb{f}=\left\{\mathbf{f}_{1}, \ldots, \mathbf{f}_{s}\right\}$ is a reduced Gröbner basis. Define

$$
\begin{aligned}
\mathcal{I}(\rho) & :=\left[\mathbb{Y}^{\mathbf{f}}-\rho(\mathbf{f}) \mid \mathbf{f} \in L_{\rho}\right] . \\
\mathcal{A}(\rho) & :=\mathbb{Y}^{\mathbf{f}_{1}}-\rho\left(\mathbf{f}_{1}\right), \ldots, y^{\mathbf{f}_{s}}-\rho\left(\mathbf{f}_{s}\right) .
\end{aligned}
$$

The Laurent binomial $\sigma$-ideal $\mathcal{I}(\rho)$ has the following properties.

Lemma 4.16 For $\rho$ and $\mathcal{A}$ defined above, $\mathcal{I}(\rho)=[\mathcal{A}(\rho)] \neq[1]$ and $\mathcal{A}(\rho)$ is a characteristic set of $\mathcal{I}(\rho)$. 
Proof: By Lemma 4.1, $\mathcal{I}(\rho)=[\mathcal{A}(\rho)]$. By Lemma 4.4, in order to prove $\mathcal{I}(\rho) \neq[1]$, it suffices to show that for any syzygy $\sum_{i} a_{i} \mathbf{f}_{i}=0$ among $\mathbf{f}_{i}$, we have $\prod_{i} \rho\left(\mathbf{f}_{i}\right)^{a_{i}}=1$. Indeed, $\rho\left(\sum_{i} a_{i} \mathbf{f}_{i}\right)=\prod_{i} \rho\left(\mathbf{f}_{i}\right)^{a_{i}}=1$, since $\rho$ is a homomorphism from the $\mathbb{Z}[x]$-module $L_{\rho}$ to $\mathcal{F}^{*}$. Since $\mathbb{f}$ is a reduced Gröber basis, by Theorem 4.10, $\mathcal{A}$ is a characteristic set of $\mathcal{I}(\rho)$.

Lemma 4.17 A Laurent $\sigma$-binomial $\mathbb{Y}^{\mathbf{f}}-c_{\mathbf{f}}$ is in $\mathcal{I}(\rho)$ if and only if $\mathbf{f} \in L_{\rho}$ and $c_{\mathbf{f}}=\rho(\mathbf{f})$.

Proof: By Lemma 4.16, $\mathcal{A}(\rho)$ is a characteristic set of $\mathcal{I}(\rho)$. Since $f=y^{\mathrm{f}}-c_{\mathrm{f}}$ is a $\sigma$-binomial in $\mathcal{I}(\rho)$, we have $r=\operatorname{prem}(f, \mathcal{A})=0$. By Lemma 4.5, $\mathbf{f}$ is in the $\mathbb{Z}[x]$-module $L_{\rho}$. The other side is obviously true and the lemma is proved.

We now show that all Laurent binomial $\sigma$-ideals are defined by partial characters.

Theorem 4.18 The map $\rho \Rightarrow \mathcal{I}(\rho)$ gives a one to one correspondence between the set of proper Laurent binomial $\sigma$-ideals and partial characters on $\mathbb{Z}[X]^{n}$.

Proof: By Lemma 4.16, a partial character defined a proper Laurent binomial $\sigma$-ideal. For the other side, let $\mathcal{I} \subseteq \mathcal{F}\left\{\mathbb{Y}^{ \pm}\right\}$be a proper Laurent binomial $\sigma$-ideal. $\mathcal{I}$ is generated by its members of the form $y^{\mathbf{f}}-c_{\mathbf{f}}$ for $\mathbf{f} \in \mathbb{Z}[x]^{n}$ and $c_{\mathbf{f}} \in \mathcal{F}^{*}$. Let $L_{\rho}=\mathbb{L}(\mathcal{I})$ which is defined in (8) and $\rho(\mathbf{f})=c_{\mathbf{f}}$. Since $\mathcal{I}$ is proper, $c_{\mathbf{f}}$ is uniquely determined by $\mathbf{f}$. By Lemma 4.1 and Corollary 4.2, $\rho$ is a partial character which is uniquely determined by $\mathcal{I}$. It is clear $\mathcal{I}(\rho)=\mathcal{I}$. To show the correspondence is one to one, it suffices to show $\rho(\mathcal{I}(\rho))=\rho$ which is a consequence of Lemma 4.17. The theorem is proved.

From Lemma 4.16] and Theorem 4.18, we have

Corollary 4.19 Any Laurent binomial $\sigma$-ideal is finitely generated.

Corollary 4.20 Let $\mathbf{f}_{i} \in \mathbb{Z}[x]^{n}$ and $c_{i} \in \mathcal{F}^{*}$ for $i=1, \ldots$, s. If $\mathcal{A}: \mathbb{Y}^{\mathbf{f}_{1}}-c_{1}, \ldots, \mathbb{Y}^{\mathbf{f}_{s}}-c_{s}$ is a regular and coherent $\sigma$-chain, then there exists a partial character $\rho$ over $\mathbb{Z}[x]^{n}$ such that $L_{\rho}=\left(\mathbf{f}_{1}, \ldots, \mathbf{f}_{s}\right), \rho\left(\mathbf{f}_{i}\right)=c_{i}$, and $\mathcal{I}(\rho)=[\mathcal{A}]$.

Proof: By Theorem 4.14, $[\mathcal{A}]$ is proper with support lattice $\left(\mathbf{f}_{1}, \ldots, \mathbf{f}_{s}\right)$. By Theorem 4.18, the corresponding partial character of $[\mathcal{A}]$ satisfies properties in the corollary.

Now, we can prove Theorem 1.1 easily.

Proof of Theorem 1.1. By Theorem 4.18, $\mathcal{I}$ is a proper Laurent binomial $\sigma$-ideal in $\mathcal{F}\left\{\mathbb{Y}^{ \pm}\right\}$if and only if $\mathcal{I}=\mathcal{I}(\rho)$ for a partial character $\rho$, hence (3). By Theorem 4.14, (1) and (2) of Theorem 1.1 are equivalent. From Lemma 4.16 and Corollary 4.9, (3) implies (2). From Corollary 4.20, (2) implies (3).

\subsection{Reflexive and prime Laurent binomial $\sigma$-ideals}

In this section, we first give criteria for reflexive and prime Laurent binomial $\sigma$-ideals and then give a decomposition theorem for perfect Laurent binomial $\sigma$-ideals. Let $a$ be an 
element in an over field of $\mathcal{F}, S$ a set of elements in an over field of $\mathcal{F}$, and $k \in \mathbb{N}$. Denote $a^{[k]}=\left\{a, a^{x}, \ldots, a^{x^{k}}\right\}$ and $S^{[k]}=\cup_{b \in S} b^{[k]}$.

For the $\sigma$-indeterminates $\mathbb{Y}=\left\{y_{1}, \ldots, y_{n}\right\}$ and $t \in \mathbb{N}$, we will treat the elements of $\mathbb{Y}^{[t]}$ as algebraic indeterminates, and $\mathcal{F}\left[\mathbb{Y}^{[t t]}\right]$ is the Laurent polynomial ring in $\mathbb{Y}^{[t]}$. Let $\mathcal{I}$ be a Laurent binomial $\sigma$-ideal in $\mathcal{F}\left\{\mathbb{Y}^{ \pm}\right\}$. Then it is easy to check that

$$
\mathcal{I}_{t}=\mathcal{I} \cap \mathcal{F}\left[\mathbb{Y}^{[ \pm t]}\right]
$$

is a Laurent binomial ideal in $\mathcal{F}\left[\mathbb{Y}^{[ \pm t]}\right]$.

Denote $\mathbb{Z}[x]_{t}$ to be the set of elements in $\mathbb{Z}[x]$ with degree $\leq t$. Then $\mathbb{Z}[x]_{t}^{n}$ is the $\mathbb{Z}$ module generated by $x^{i} \boldsymbol{\epsilon}_{l}$ for $i=0, \ldots, t, l=1, \ldots, n$. It is clear that $\mathbb{Z}[x]_{t}^{n}$ is isomorphic to $\mathbb{Z}^{n(t+1)}$ as $\mathbb{Z}$-modules by mapping $x^{i} \boldsymbol{\epsilon}_{l}$ to the $((l-1)(t+1)+i+1)$-th standard basis vector in $\mathbb{Z}^{n(t+1)}$. Hence, we treat them as the same in this section. Let $L$ be a $\mathbb{Z}[x]$-lattice and $t \in \mathbb{N}$. Then

$$
L_{t}=L \cap \mathbb{Z}[x]_{t}^{n}=L \cap \mathbb{Z}^{n(t+1)}
$$

is a $\mathbb{Z}$-module in $\mathbb{Z}^{n(t+1)}$. Similarly, it can be shown that when restricting to $\mathbb{Z}[x]_{t}^{n}$, a partial character $\rho$ on $\mathbb{Z}[x]^{n}$ becomes a partial character $\rho_{t}$ on $\mathbb{Z}^{n(t+1)}$.

Lemma 4.21 With the notations introduced above, we have $\mathcal{I}_{t}=\mathcal{I} \cap \mathcal{F}\left[\mathbb{Y}^{[ \pm t]}\right]=\mathcal{I}\left(\rho_{t}\right)$.

Proof: It suffices to show that the support lattice of $\mathcal{I}_{t}$ is $L_{\rho_{t}}=L_{t}$. By Lemma 4.17, $\mathbb{Y}^{\mathbf{f}}-c_{m} \in \mathcal{I}_{t}$ if and only if $\mathbf{f} \in L \cap \mathbb{Z}[x]_{t}^{n}$, or equivalently, $\max _{m \in \mathbf{f}} \operatorname{deg}(m, x) \leq t$, which is equivalent to $\mathbf{f} \in L_{t}$.

Definition 4.22 Let $L$ be a $\mathbb{Z}[x]$-module in $\mathbb{Z}[x]^{n}$.

- $L$ is called $\mathbb{Z}$-saturated if, for any $a \in \mathbb{Z}$ and $\mathbf{f} \in \mathbb{Z}[x]^{n}$, af $\in L$ implies $\mathbf{f} \in L$.

- $L$ is called $x$-saturated if, for any $\mathbf{f} \in \mathbb{Z}[x]^{n}, x \mathbf{f} \in L$ implies $\mathbf{f} \in L$.

- $L$ is called saturated if it is both $\mathbb{Z}$ - and $x$ - saturated.

We now prove (1)-(2) of Theorem 1.2, that is

Theorem 4.23 Let $\rho$ be a partial character over $\mathbb{Z}[x]^{n}$. If $\mathcal{F}$ is algebraically closed and inversive, then

(a) $L_{\rho}$ is $\mathbb{Z}$-saturated if and only if $\mathcal{I}(\rho)$ is prime;

(b) $L_{\rho}$ is $x$-saturated if and only if $\mathcal{I}(\rho)$ is reflexive;

(c) $L_{\rho}$ is saturated if and only if $\mathcal{I}(\rho)$ is reflexive prime.

Proof: It is clear that (c) comes from (a) and (b). Let $\mathcal{I}=\mathcal{I}(\rho)$ and $L=L_{\rho}$.

$(a): \mathcal{I}$ is a Laurent prime $\sigma$-ideal if and only if $\mathcal{I}_{t}$ is a Laurent prime ideal for all $t$. From Lemma 4.21, the support of $\mathcal{I}_{t}$ is $L_{t}$. Then by [7, Thm 2.1], $\mathcal{I}_{t}$ is a Laurent prime ideal if 
and only if $L_{t}$ is a $\mathbb{Z}$-saturated $\mathbb{Z}$-module. Furthermore, a $\mathbb{Z}[x]$-lattice $L$ is $\mathbb{Z}$-saturated if and only if $L_{t}$ is a $\mathbb{Z}$-saturated $\mathbb{Z}$-module for all $t$. Thus, (a) is valid.

(b): Suppose $\mathcal{I}$ is reflexive. For $x \mathbf{f} \in L$, by Lemma 4.17, there is a $\mathbb{Y}^{x \mathbf{f}}-c \in \mathcal{I}$. Since $\mathcal{F}$ is reflexive, $c=d^{x}$ for $d \in \mathcal{F}$. Then $\sigma\left(\mathbb{Y}^{\mathbf{f}}-d\right) \in \mathcal{I}$ and hence $\mathbb{Y}^{\mathbf{f}}-d \in \mathcal{I}$ since $\mathcal{I}$ is reflexive. By Lemma 4.17 again, $\mathbf{f} \in L$ and $L$ is $x$-saturated. To prove the other direction, assume $L$ is $x$-saturated. For $f^{x} \in \mathcal{I}$, we have an expression

$$
f^{x}=\sum_{i=1}^{s} f_{i}\left(\mathbb{Y}^{\mathbf{f}_{i}}-c_{i}\right)
$$

where $\mathbb{Y}^{\mathbf{f}_{i}}-c_{i} \in \mathcal{I}$ and $f_{i} \in \mathcal{F}\left\{\mathbb{Y}^{ \pm}\right\}$. Let $d=\max _{i=1}^{s} \operatorname{deg}\left(\mathbb{Y}^{\mathbf{f}_{i}}-c_{i}, y_{1}\right)$ and assume $\mathbb{Y}^{\mathbf{f}_{1}}=M_{1} y_{1}^{d}$. Replace $y_{1}^{d}$ by $c_{1} / M_{1}$ in (18). Since (18) is an identity for the variables $y_{i}^{(j)}$, this replacement is meaningful and we obtain a new identity. $\mathbb{Y}^{\mathbf{f}_{1}}-c_{1}$ becomes zero after the replacement. Due to the way to chose $d$, if another summand, say $\mathbb{Y}^{\mathbf{f}_{2}}-c_{2}$, is affected by the replacement, then $\mathbb{Y}^{\mathbf{f}_{2}}=M_{2} y_{1}^{d}$. After the replacement, $\mathbb{Y}^{\mathbf{f}_{2}}-c_{2}$ becomes $c_{1}\left(M_{2} / M_{1}-c_{2} / c_{1}\right)$ which is also in $\mathcal{I}$ by Lemma 4.17. In summary, after the replacement, the right hand side of (18) has less than $s$ summands and the left hand side of (18) does not changed. Repeat the above procedure, we will eventually obtain a new indenity

$$
f^{x}=\sum_{i=1}^{\bar{s}} \bar{f}_{i}\left(\mathbb{Y}^{x \mathbf{g}_{i}}-\bar{c}_{i}\right)
$$

where $\mathbb{Y}^{x \mathbf{g}_{i}}-\bar{c}_{i} \in \mathcal{I}$ and $\bar{f}_{i} \in \mathcal{F}\left\{\mathbb{Y}^{ \pm}\right\}$. We may assume that any $y_{i}$ does not appear in $\bar{f}_{i}$. Otherwise, by setting $y_{i}$ to be 1 , the left hand side of (19) is not changes and a new identity is obtained. Since $\mathcal{F}$ is inversive, $\bar{c}_{i}=e_{i}^{x}$ and $\bar{f}_{i}=g_{i}^{x}$ for $e_{i} \in \mathcal{F}$ and $g_{i} \in \mathcal{F}\left\{\mathbb{Y}^{ \pm}\right\}$. By Lemma 4.17, $\mathbb{Y}^{x \mathbf{g}_{i}}-e_{i}^{x} \in I$ implies $x \mathbf{g}_{i} \in L$. Since $L$ is $x$-saturated, $x \mathbf{g}_{i} \in L$ implies $\mathbf{g}_{i} \in L$ and hence $\mathbb{Y}^{\mathbf{g}_{i}}-e_{i} \in I$ by Lemma 4.17 again. From (19), $\sigma\left(f-\sum_{i=1}^{\bar{s}} g_{i}\left(\mathbb{Y}^{\mathbf{g}_{i}}-e_{i}\right)\right)=0$ and hence $f=\sum_{i=1}^{\bar{s}} g_{i}\left(\mathbb{Y}^{\mathbf{g}_{i}}-e_{i}\right) \in \mathcal{I}$. (b) is proved.

Decision procedures for whether a $\mathbb{Z}[x]$-lattice is $\mathbb{Z}$-saturated or $x$-saturated will be given in Section 7

From Corollary 4.13, every proper Laurent binomial $\sigma$-ideal is radical. The following example shows that a binomial $\sigma$-ideal is not necessarily perfect.

Example 4.24 Let $\mathcal{I}=[\mathcal{A}] \subset \mathbb{Q}\left\{y_{1}, y_{2}\right\}$, where $\mathcal{A}=\left\{y_{1}^{2}+1, y_{1}^{x}-y_{1}, y_{2}^{2}+1, y_{2}^{x}+y_{2}\right\}$ is a $\sigma$ chain. We have $y_{2}^{2}+1-\left(y_{1}^{2}+1\right)=\left(y_{2}-y_{1}\right)\left(y_{2}+y_{1}\right)$. Then $\{\mathcal{I}\}=\left\{\mathcal{I}, y_{2}-y_{1}\right\} \cap\left\{\mathcal{I}, y_{2}+y_{1}\right\}=[1]$ since $\left[\mathcal{I}, y_{2}-y_{1}\right]=\left[\mathcal{I}, y_{2}+y_{1}\right]=[1]$. On the other hand, $1 \notin \mathcal{I}$, since $\mathcal{A}$ is a characteristic set of $\mathcal{I}$ by Theorem 4.10 .

Definition 4.25 Let $L \subset \mathbb{Z}[x]^{n}$ be a $\mathbb{Z}[x]$-lattice. The $\mathbb{Z}$-saturation of $L$ is $\operatorname{sat}_{\mathbb{Z}}(L)=\{\mathbf{f} \in$ $\mathbb{Z}[x]^{n} \mid \exists a \in \mathbb{Z}$ s.t. af $\left.\in L\right\}$. The $x$-saturation of $L$ is $\operatorname{sat}_{x}(L)=\left\{\mathbf{f} \in \mathbb{Z}[x]^{n} \mid x \mathbf{f} \in L\right\}$. The saturation of $L$ is $\operatorname{sat}(L)=\left\{\mathbf{f} \in \mathbb{Z}[x]^{n} \mid \exists a \in \mathbb{Z}, \exists k \in \mathbb{N}\right.$ s.t. $\left.a x^{k} \mathbf{f} \in L\right\}$.

It is clear that the $\mathbb{Z}$-saturation ( $x$-saturation) of $L$ is $\mathbb{Z}$-saturated ( $x$-saturated) and

$$
\operatorname{sat}(L)=\operatorname{sat}_{\mathbb{Z}}\left(\operatorname{sat}_{x}(L)\right)=\operatorname{sat}_{x}\left(\operatorname{sat}_{\mathbb{Z}}(L)\right) .
$$


Algorithms to compute the $\mathbb{Z}$-saturation and $x$-saturation of a $\mathbb{Z}[x]$-lattice will be given in Section 7 .

Theorem 4.26 Let $\mathcal{I}$ be a Laurent binomial $\sigma$-ideal and $L$ the support lattice of $\mathcal{I}$. If $\mathcal{F}$ is inversive, then the reflexive closure of $\mathcal{I}$ is also a Laurent binomial $\sigma$-ideal whose support lattice is the $x$-saturation of $L$.

Proof: Let $\mathcal{I}_{x}$ be the reflexive closure of $\mathcal{I}$ and $L_{x}=\operatorname{sat}_{x}(L)$. Suppose $\mathcal{I}=\left[f_{1}, \ldots, f_{r}\right]$, where $f_{i}=\mathbb{Y}^{\mathbf{f}_{i}}-c_{i}$. Then $L=\left(\mathbf{f}_{1}, \ldots, \mathbf{f}_{r}\right)$. If $L$ is $x$-saturated, by Theorem 4.23, $\mathcal{I}$ is reflexive. Otherwise, there exist $k_{1} \in \mathbb{N}, b_{i} \in \mathbb{Z}[x]$, and $\mathbf{h}_{1} \in \mathbb{Z}[x]^{n}$ such that $\mathbf{h}_{1} \notin L$ and

$$
x^{k_{1}} \mathbf{h}_{1}=\sum_{i=1}^{r} b_{i} \mathbf{f}_{i} \in L .
$$

By Lemma 4.1, $\mathbb{Y}^{x^{k_{1}} \mathbf{h}_{1}}-\widetilde{a}$ is in $\mathcal{I}$, where $\widetilde{a}=\prod_{i=1}^{r} c_{i}^{b_{i}}$. Since $\mathcal{F}$ is inversive, $\bar{a}=\sigma^{-k_{1}}(\widetilde{a}) \in \mathcal{F}$. Then, $\sigma^{k_{1}}\left(\mathbb{Y}^{\mathbf{h}_{1}}-\bar{a}\right) \in \mathcal{I}$, and hence $\mathbb{Y}^{\mathbf{h}_{1}}-\bar{a} \in \mathcal{I}_{x}$. Let $\mathcal{I}_{1}=\left[f_{1}, \ldots, f_{r}, \mathbb{Y}^{\mathbf{h}_{1}}-\bar{a}\right]$. It is clear that $L_{1}=\left(\mathbf{f}_{1}, \ldots, \mathbf{f}_{r}, \mathbf{h}_{1}\right)$ is the support lattice of $\mathcal{I}_{1}$. Then $\mathcal{I} \varsubsetneqq \mathcal{I}_{1} \subset \mathcal{I}_{x}$ and $L \varsubsetneqq L_{1} \subset L_{x}$. Repeating the above procedure for $\mathcal{I}_{1}$ and $L_{1}$, we obtain $\mathcal{I}_{2}$ and $L_{2}=$ $\left(\mathbf{f}_{1}, \ldots, \mathbf{f}_{r}, \mathbf{h}_{1}, \mathbf{h}_{2}\right)$ such that $\mathbf{h}_{2} \notin L_{1}$ and $x^{k_{2}} \mathbf{h}_{2} \in L_{1}$. We claim that $L_{2} \subset L_{x}$. Indeed, let $x^{k_{2}} \mathbf{h}_{2}=\sum_{i=1}^{r} e_{i} \mathbf{f}_{i}+e_{0} \mathbf{h}_{1}$. Then by (20), $x^{k_{1}+k_{2}} \mathbf{h}_{2}=x^{k_{1}}\left(x^{k_{2}} \mathbf{h}_{2}\right)=x^{k_{1}} \sum_{i=1}^{r} e_{i} \mathbf{f}_{i}+e_{0}\left(x^{k_{1}} \mathbf{h}_{1}\right)=$ $x^{k_{1}} \sum_{i=1}^{r} e_{i} \mathbf{f}_{i}+e_{0} \sum_{i=1}^{r} b_{i} \mathbf{f}_{i} \in L$ and the claim is proved. As a consequence, $\mathcal{I}_{2} \subset \mathcal{I}_{x}$.

Continuing the process, we have $\mathcal{I} \varsubsetneqq \mathcal{I}_{1} \varsubsetneqq \cdots \varsubsetneqq \mathcal{I}_{t} \subset \mathcal{I}_{x}$ and $L \varsubsetneqq L_{1} \varsubsetneqq \cdots \varsubsetneqq L_{t} \subset L_{x}$ such that $L_{i}$ is the support lattice of $\mathcal{I}_{i}$. The process will terminate, since $\mathbb{Z}[x]^{n}$ is Northerian. The final $\mathbb{Z}[x]$-lattice $L_{t}$ is $x$-saturated and hence $\mathcal{I}_{t}$ is reflexive by Theorem 4.23, Since $L_{x}$ is the smallest $x$-saturated $\mathbb{Z}[x]$-lattice containing $L$ and $L \subset L_{t} \subset L_{x}$, we have $L_{t}=L_{x}$ and $\mathcal{I}_{t}=\mathcal{I}_{x}$.

Corollary 4.27 Let $L \subset \mathbb{Z}[x]^{n}$ be a $\mathbb{Z}[x]$-lattice. Then $L_{x}=\operatorname{sat}_{x}(L)$ is generated by $L$ and a finite number of elements which are linear combinations of elements of $L$ divided by certain $x^{d}$. Furthermore, $\operatorname{rk}(L)=\operatorname{rk}\left(L_{x}\right)$.

Proof: From the proof of Theorem 4.26, $\operatorname{sat}_{x}(L)=\left(L, \mathbf{h}_{1}, \ldots, \mathbf{h}_{t}\right)$ and for each $\mathbf{h}_{i}$, there is a positive integer $n_{i}$ such that $x^{n_{i}} \mathbf{h}_{i} \in L$. Let $A$ be a representation matrix of $L$. Then a representation matrix $B$ of $L_{x}$ can be obtained by adding to $A$ a finite number of new columns which are linear combinations of columns of $A$ divided by some $x^{d}$. Therefore, $\operatorname{rk}(A)=\operatorname{rk}(B)$.

Similarly, we can show

Lemma 4.28 Let $L \subset \mathbb{Z}[x]^{n}$ be a $\mathbb{Z}[x]$-lattice. Then $L_{\mathbb{Z}}=\operatorname{sat}_{\mathbb{Z}}(L)$ is generated by $L$ and a finite number of elements which are linear combinations of elements of $L$ divided by an integer. Furthermore, $\operatorname{rk}(L)=\operatorname{rk}\left(L_{\mathbb{Z}}\right)$.

By Corollary 4.13, a Laurent binomial $\sigma$-ideal $\mathcal{I}$ is radical. By Example 4.24, $\mathcal{I}$ is not necessarily perfect. We now give a decomposition theorem for perfect $\sigma$-ideals. 
Theorem 4.29 Let $\mathcal{I}$ be a Laurent binomial $\sigma$-ideal, $L$ the support lattice of $\mathcal{I}$, and $L_{S}$ the saturation of $L$. If $\mathcal{F}$ is algebraically closed and inversive, then $\{\mathcal{I}\}$ is either [1] or can be written as the intersection of Laurent reflexive prime binomial $\sigma$-ideals whose support lattice is $L_{S}$.

Proof: Let $\mathcal{I}_{x}$ be the reflexive closure of $\mathcal{I}$ and $L_{x}=\operatorname{sat}_{x}(L)$. By Theorem 4.26, $L_{x}$ is the support lattice of $\mathcal{I}_{x}$. Suppose $\mathcal{I}_{x}=\left[f_{1}, \ldots, f_{r}\right], f_{i}=\mathbb{Y}^{\mathbf{f}_{i}}-c_{i}, i=1, \ldots, r$, and $L_{x}=$ $\left(\mathbf{f}_{1}, \ldots, \mathbf{f}_{r}\right)$. If $L_{x}$ is $\mathbb{Z}$-saturated, then by Theorem 4.23, $\mathcal{I}_{x}$ is reflexive prime. Otherwise, there exist $k_{1} \in \mathbb{N}, a_{i} \in \mathbb{Z}[x]$, and $\mathbf{h}_{1} \in \mathbb{Z}[x]^{n}$ such that $\mathbf{h}_{1} \notin L_{x}$ and

$$
k_{1} \mathbf{h}_{1}=a_{1} \mathbf{f}_{1}+\cdots+a_{r} \mathbf{f}_{r} \in L_{x} .
$$

By Lemma 4.1, $\mathbb{Y}^{k_{1} \mathbf{h}_{1}}-\widetilde{a} \in \mathcal{I}$, where $\widetilde{a}=\prod_{i=1}^{r} c_{i}^{a_{i}}$. Since $\mathcal{F}$ is algebraically closed, we have

$$
\mathbb{Y}^{k_{1} \mathbf{h}_{1}}-\widetilde{a}=\prod_{l=1}^{k_{1}}\left(\mathbb{Y}^{\mathbf{h}_{1}}-\widetilde{a}_{l}\right) \in \mathcal{I}_{x}
$$

where $\widetilde{a}_{l}, l=1, \ldots, k_{1}$ are the $k_{1}$ roots of $\widetilde{a}$. By the difference Nullstellensatz [3, p.87], we have the following decomposition

$$
\{\mathcal{I}\}=\cap_{l_{1}=1}^{k_{1}}\left\{\mathcal{I}_{1 l_{1}}\right\}
$$

where $\mathcal{I}_{1 l}=\left[f_{1}, \ldots, f_{r}, \mathbb{Y}^{\mathbf{h}_{1}}-\widetilde{a}_{l}\right]$. Check whether $\mathcal{I}_{1 l_{1}}=[1]$ with Lemma 4.4 and discard those trivial ones. Then the support lattice for any of $\mathcal{I}_{1 l}$ is $L_{1}=\left(\mathbf{f}_{1}, \ldots, \mathbf{f}_{r}, \mathbf{h}_{1}\right)$. Similar to the proof of Theorem 4.26, we can show that $\mathcal{I}_{x} \varsubsetneqq \mathcal{I}_{1 l}$ and $L_{x} \varsubsetneqq L_{1} \subset L_{S}$.

Repeating the process, we have $\mathcal{I}_{x} \varsubsetneqq \mathcal{I}_{1 l_{1}} \varsubsetneqq \cdots \varsubsetneqq \mathcal{I}_{t l_{t}}$ for $l_{i}=1, \ldots, k_{i}$ and $L_{x} \varsubsetneqq L_{1} \varsubsetneqq$ $L_{2} \varsubsetneqq \cdots \varsubsetneqq L_{t} \subset L_{S}$ such that $L_{i}$ is the support lattice of $\mathcal{I}_{i l_{i}}$ for $l_{i}=1, \ldots, k_{i}$ and

$$
\{\mathcal{I}\}=\cap_{l_{i}=1}^{k_{i}}\left\{\mathcal{I}_{i l_{i}}\right\}, i=1, \ldots, t .
$$

The process will terminate, since $\mathbb{Z}[x]^{n}$ is Northerian. Since $L_{S}$ is the smallest $\mathbb{Z}$-saturated $\mathbb{Z}[x]$-lattice containing $L_{x}$ and $L_{x} \subset L_{t} \subset L_{S}$, we have $L_{t}=\operatorname{sat}_{\mathbb{Z}}\left(L_{x}\right)=\operatorname{sat}_{\mathbb{Z}}\left(\operatorname{sat}_{x}(L)\right)=L_{S}$. Then $\mathcal{I}_{t l_{t}}$ is reflexive prime and the theorem is proved.

Since the reflexive prime components of $\mathcal{I}$ have the same support lattice, they also have the same dimension.

Corollary 4.30 Any Laurent binomial $\sigma$-ideal $\mathcal{I}$ is dimensionally unmixed.

The condition for $\mathcal{F}$ to be algebraically closed and inversive is necessary for Theorem 4.29 to be valid. For instance, if $\mathcal{F}=\mathbb{Q}(\lambda)$ and $\sigma(f(\lambda))=f\left(\lambda^{2}\right)$, then $\left[y_{1}^{2} y_{2}^{2}-\lambda\right]$ is prime and its support lattice $\left([2,2]^{\tau}\right)$ is not $\mathbb{Z}$-saturated. Furthermore, $\left[y_{1}^{x} y_{2}^{x}-\lambda\right]$ is reflexive and its support lattice $\left([x, x]^{\tau}\right)$ is not $x$-saturated. 


\subsection{Well-mixed and perfect Laurent binomial $\sigma$-ideals}

In this section, we give a criterion for a Laurent binomial $\sigma$-ideal to be well-mixed and perfect in terms of its support lattice and show that the well-mixed and perfect closures of a Laurent binomial $\sigma$-ideal are still binomial.

Recall that a $\sigma$-ideal $\mathcal{I}$ is called well-mixed if $f g \in \mathcal{I}$ implies $f g^{x} \in \mathcal{I}$ for $f, g \in \mathcal{F}\left\{\mathbb{Y}^{ \pm}\right\}$. The smallest well-mixed $\sigma$-ideal containing $S \subset \mathcal{F}\left\{\mathbb{Y}^{ \pm}\right\}$is denoted by $\langle S\rangle$. Let $S^{\prime}=$ $\left\{f g^{x} \mid f g \in S\right\}$. We define inductively: $S_{0}=S, S_{n}=\left[S_{n-1}\right]^{\prime}, n=1,2, \ldots$ The union of the $S_{n}$ is clearly a well-mixed $\sigma$-ideal and is contained in every well-mixed $\sigma$-ideal containing $S$. Hence this union is $\langle S\rangle$. If $\mathcal{I} \subset \mathcal{F}\left\{\mathbb{Y}^{ \pm}\right\}$is a Laurent $\sigma$-ideal, then $\langle\mathcal{I}\rangle$ ia called the well-mixed closure of $\mathcal{I}$. We first prove some basic properties of well-mixed $\sigma$-ideals which will be used later. Note that these properties are also valid in $\mathcal{F}\{\mathbb{Y}\}$.

Lemma 4.31 Let $\mathcal{I}_{1}, \ldots, \mathcal{I}_{s}$ be prime $\sigma$-ideals. Then $\mathcal{I}=\cap_{i=1}^{s} \mathcal{I}_{i}$ is a well-mixed $\sigma$-ideal.

Proof: It is obvious.

Lemma 4.32 Let $S_{1}, S_{2}$ be two subsets of $\mathcal{F}\left\{\mathbb{Y}^{ \pm}\right\}$which satisfy a $\in S_{i}$ implies $\sigma(a) \in S_{i}, i=$ 1,2. Then $\left[S_{1}\right]_{n}\left[S_{2}\right]_{n} \subset\left[S_{1} S_{2}\right]_{n}$.

Proof: Let $s \in\left[S_{1}\right]_{1}$ and $t \in\left[S_{2}\right]_{1}$. Then $s=f_{1} g_{1}^{x}$ and $t=f_{2} g_{2}^{x}$ where $f_{1} g_{1}, f_{2} g_{2}$ are linear combinations of members of $S_{1}$ and $S_{2}$, respectively. Then, $f_{1} g_{1} f_{2} g_{2} \in\left[S_{1} S_{2}\right]$, and st $=f_{1} f_{2}\left(g_{1} g_{2}\right)^{x} \in\left[S_{1} S_{2}\right]_{1}$. Hence, $\left[S_{1}\right]_{1}\left[S_{2}\right]_{1} \subset\left[S_{1} S_{2}\right]_{1}$. By induction, $\left[S_{1}\right]_{n}\left[S_{2}\right]_{n} \subset\left[S_{1} S_{2}\right]_{n}$.

Lemma 4.33 Let $S_{1}, S_{2}$ be two subsets of $\mathcal{F}\left\{\mathbb{Y}^{ \pm}\right\}$which satisfy a $\in S_{i}$ implies $\sigma(a) \in S_{i}, i=$ 1,2. Then $\sqrt{\left[S_{1} S_{2}\right]_{n}}=\sqrt{\left[S_{1}\right]_{n} \cap\left[S_{2}\right]_{n}}$ for $n \geq 1$, and $\sqrt{\left\langle S_{1}\right\rangle} \cap \sqrt{\left\langle S_{2}\right\rangle}=\sqrt{\left\langle S_{1} S_{2}\right\rangle}$.

Proof: The last statement is an immediate consequence of the first one. Since $\left[S_{1} S_{2}\right] \subset\left[S_{i}\right]$, we have $\left[S_{1} S_{2}\right]_{n} \subset\left[S_{i}\right]_{n}$ for $i=1,2$, and $\left[S_{1} S_{2}\right]_{n} \subset\left[S_{1}\right]_{n} \cap\left[S_{2}\right]_{n}$ follows. Hence, $\sqrt{\left[S_{1} S_{2}\right]_{n}} \subset$ $\sqrt{\left[S_{1}\right]_{n} \cap\left[S_{2}\right]_{n}}$. Let $a \in\left[S_{1}\right]_{n} \cap\left[S_{2}\right]_{n}$ we have $a^{2} \in\left[S_{1}\right]_{n}\left[S_{2}\right]_{n}$. By Lemma44.32, $a^{2} \in\left[S_{1} S_{2}\right]_{n}$. Hence $a \in \sqrt{\left[S_{1} S_{2}\right]_{n}}$, and $\sqrt{\left[S_{1}\right]_{n} \cap\left[S_{2}\right]_{n}} \subset \sqrt{\left[S_{1} S_{2}\right]_{n}}$ follows.

Lemma 4.34 Let $\mathcal{I}_{1}, \ldots, \mathcal{I}_{m}$ be Laurent $\sigma$-ideals. Then $\sqrt{\left\langle\cap_{i=1}^{m} \mathcal{I}_{i}\right\rangle}=\cap_{i=1}^{m} \sqrt{\left\langle\mathcal{I}_{i}\right\rangle}$.

Proof: Let $\mathcal{I}=\cap_{i=1}^{m} \mathcal{I}_{i}$. Then $\sqrt{\mathcal{I}}=\sqrt{\left[\prod_{i=1}^{m} \mathcal{I}_{i}\right]}$. By Lemma 4.33, we have $\sqrt{\left\langle\prod_{i=1}^{m} \mathcal{I}_{i}\right\rangle}=$ $\sqrt{\prod_{i=1}^{n-1}\left\langle\mathcal{I}_{i}\right\rangle} \cap \sqrt{\left\langle\mathcal{I}_{n}\right\rangle}=\ldots=\cap_{i=1}^{m} \sqrt{\left\langle\mathcal{I}_{i}\right\rangle}$. Now we show that $\sqrt{\langle\mathcal{I}\rangle}=\sqrt{\left\langle\prod_{i=1}^{m} \mathcal{I}_{i}\right\rangle}$. Since $\prod_{i=1}^{m} \mathcal{I}_{i} \subset \mathcal{I}$, we have $\sqrt{\left\langle\prod_{i=1}^{m} \mathcal{I}_{i}\right\rangle} \subset \sqrt{\langle\mathcal{I}\rangle}$. By Lemma 4.33, $\sqrt{\langle I\rangle}=\sqrt{\langle I\rangle} \cap \cdots \cap \sqrt{\langle I\rangle}=$ $\sqrt{\left\langle I^{m}\right\rangle} \subset \sqrt{\left\langle\prod_{i=1}^{m} \mathcal{I}_{i}\right\rangle}$, and hence $\sqrt{\langle\mathcal{I}\rangle}=\sqrt{\left\langle\prod_{i=1}^{m} \mathcal{I}_{i}\right\rangle}$. Then, $\sqrt{\langle\mathcal{I}\rangle}=\cap_{i=1}^{m} \sqrt{\left\langle\mathcal{I}_{i}\right\rangle}$.

Now, we prove a basic property for a $\sigma$-field $\mathcal{F}$. 
Lemma 4.35 Let $\zeta_{m}=e^{\frac{2 \pi \mathrm{i}}{m}}$ be the primitive $m$-th root of unity, where $\mathrm{i}=\sqrt{-1}$ and $m \in$ $\mathbb{Z}_{\geq 2}$. If $\mathcal{F}$ is an algebraically closed $\sigma$-field, then there exists an $o_{m} \in[0, m-1]$ such that $\operatorname{gcd}\left(o_{m}, m\right)=1$ and $\sigma\left(\zeta_{m}\right)=\zeta_{m}^{o_{m}}$. Furthermore, the perfect $\sigma$-ideal $\left\{y^{m}-1\right\}$ in $\mathcal{F}\{y\}$ is

$$
\left\{y^{m}-1\right\}=\left[y^{m}-1, y^{x}-y^{o m}\right]
$$

where $y$ is a $\sigma$-indeterminate.

Proof: Since $\mathcal{F}$ is algebraically closed, $\zeta_{m}$ is in $\mathcal{F}$. From $y^{m}-1=\prod_{j=0}^{m-1}\left(y-\zeta_{m}^{j}\right)=0$, we have $\sigma(y)^{m}-1=\prod_{j=0}^{m-1}\left(\sigma(y)-\zeta_{m}^{j}\right)=0$. Then, there exists an $o_{m}$ such that $0 \leq o_{m} \leq m-1$ and $\sigma\left(\zeta_{m}\right)=\zeta_{m}^{o_{m}}$. Suppose $\operatorname{gcd}\left(o_{m}, m\right)=d>1$ and let $o_{m}=d k, m=d s$, where $s \in[1, m-1]$. Then $\sigma\left(\zeta_{m}^{s}\right)=\zeta_{m}^{o_{m} s}=\zeta_{m}^{d k s}=\zeta_{m}^{k m}=1$, which implies $\zeta_{m}^{s}=1$, a contradiction.

By the difference Nullstellensatz [3, p.87], we have $\left\{y^{m}-1\right\}=\cap_{j=0}^{m-1}\left[y-\zeta_{m}^{j}\right]$. In order to show (22), it suffices to show $\cap_{j=0}^{m-1}\left[y-\zeta_{m}^{j}\right]=\left[y^{m}-1, y^{x}-y^{o_{m}}\right]$. Since $y^{x}-y^{o_{m}}=$ $\left(y-\zeta_{m}^{j}\right)^{x}+\zeta_{m}^{x j}-y^{o_{m}}=\left(y-\zeta_{m}^{j}\right)^{x}+\zeta_{m}^{j o_{m}}-y^{o_{m}} \in\left[y-\zeta_{m}^{j}\right]$ for any $0 \leq j \leq m-1$, we have $y^{x}-y^{o_{m}} \in \cap_{j=0}^{m-1}\left[y-\zeta_{m}^{j}\right]$ and hence $\left[y^{m}-1, y^{x}-y^{o_{m}}\right] \subset \cap_{j=0}^{m-1}\left[y-\zeta_{m}^{j}\right]$. Let $f \in \cap_{j=0}^{m-1}\left[y-\zeta_{m}^{j}\right]$. Since $y^{x}-y^{o_{m}} \in\left[y-\zeta_{m}^{j}\right]$, for $j=0, \ldots, m-1$, from $f \in\left[y-\zeta_{m}^{j}\right]$, we have $f=g_{j}\left(y-\zeta_{m}^{j}\right)+\sum_{k} h_{j k}\left(y^{x}-y^{o_{m}}\right)^{x^{k}}$, where $g_{j}, h_{j k} \in \mathbb{Q}\{y\}$. Then $f^{m}=\prod_{j=0}^{m-1}\left(g_{j}\left(y-\zeta_{m}^{j}\right)+\right.$ $\left.\sum_{k} h_{j k}\left(y^{x}-y^{o_{m}}\right)^{k x}\right)=\prod_{j=0}^{m-1} g_{j}\left(y^{m}-1\right)+p$, where $p \in\left[y^{x}-y^{o_{m}}\right]$. Hence, $f \in\left[y^{m}-1, y^{x}-y^{o_{m}}\right]$ and $\cap_{j=0}^{m-1}\left[y-\zeta_{m}^{j}\right] \subset\left[y^{m}-1, y^{x}-y^{o_{m}}\right]$. The lemma is proved.

The number $o_{m}$ introduced in Lemma 4.35 depends on $\mathcal{F}$ only and is called the $n$-th transforming degree of unity. In the following corollaries, $\mathcal{F}$ is assumed to be algebraically closed and hence $o_{m}$ is fixed for any $m \in \mathbb{N}$. From the proof of Lemma 4.35, we have

Corollary 4.36 $y^{x}-y^{o_{m}} \in \cap_{j=0}^{m-1}\left[y-\zeta_{m}^{j}\right]$.

Corollary 4.37 For $n, m, k$ in $\mathbb{N}$, if $n=k m$ then $o_{n}=o_{m} \bmod m$.

Proof: By definition, $\zeta_{n}^{k}=\zeta_{m}$. Then, $\sigma\left(\zeta_{n}^{k}\right)=\zeta_{n}^{k o_{n}}=\zeta_{m}^{o_{n}}$. From, $\sigma\left(\zeta_{n}^{k}\right)=\sigma\left(\zeta_{m}\right)=\zeta_{m}^{o_{m}}$, we have $\zeta_{m}^{o_{n}}=\zeta_{m}^{o_{m}}$. Then $o_{n}=o_{m} \bmod m$.

Lemma 4.38 $\left\langle y^{m}-1\right\rangle=\left\{y^{m}-1\right\}=\left[y^{m}-1, y^{x}-y^{o_{m}}\right]$.

Proof: By Lemma 4.35, it suffices to show $y^{x}-y^{o m} \in\left\langle y^{m}-1\right\rangle$. Since $y^{m}-1=\prod_{j=0}^{m-1}\left(y-\zeta_{m}^{j}\right)$ and $\left(y-\zeta_{m}^{i}\right)^{x}=\left(y^{x}-\zeta_{m}^{o_{m} i}\right)$, we have $f_{i}=\left(y^{x}-\zeta_{m}^{o_{m} i}\right) \prod_{0 \leq j \leq m-1, j \neq i}\left(y-\zeta_{m}^{j}\right) \in\left\langle y^{m}-1\right\rangle$ for $i=0, \ldots, m-1$. We will show that $y^{x}-y^{o_{m}} \in\left(f_{0}, \ldots, f_{m-1}\right)$. To show this, we need the 
formula $\frac{1}{y^{m}-1}=\sum_{i=0}^{m-1} \frac{1}{m\left(\zeta_{m}^{i}\right)^{m-1}\left(y-\zeta_{m}^{i}\right)}=\frac{1}{m} \sum_{i=0}^{m-1} \frac{\zeta_{m}^{i}}{y-\zeta_{m}^{i}}$ from [14, p. 494]. We have

$$
\begin{aligned}
\frac{1}{m} \sum_{i=0}^{m-1} \zeta_{m}^{i} f_{i} & =\frac{1}{m} \sum_{i=0}^{m-1} \zeta_{m}^{i} \frac{y^{m}-1}{y-\zeta_{m}^{i}}\left(y^{x}-\zeta_{m}^{o_{m} i}\right) \\
& =\frac{1}{m} \sum_{i=0}^{m-1} \zeta_{m}^{i} \frac{y^{m}-1}{y-\zeta_{m}^{i}} y^{x}-\frac{1}{m} \sum_{i=0}^{m-1} \zeta_{m}^{i} \frac{y^{m}-1}{y-\zeta_{m}^{i}} \zeta_{m}^{o_{m} i} \\
& =y^{x}-\frac{1}{m} \sum_{i=0}^{m-1} \frac{y^{m}-1}{y-\zeta_{m}^{i}} \zeta_{m}^{\left(o_{m}+1\right) i}
\end{aligned}
$$

Let $g(y)=\frac{1}{m} \sum_{i=0}^{m-1} \frac{y^{m}-1}{y-\zeta_{m}^{i}} \zeta_{m}^{\left(o_{m}+1\right) i}$. Then, $g\left(\zeta_{m}^{j}\right)=\left.\frac{1}{m} \zeta_{m}^{\left(o_{m}+1\right) j} \frac{y^{m}-1}{y-\zeta_{m}^{j}}\right|_{y=\zeta_{m}^{j}}=\frac{1}{m} \zeta_{m}^{\left(o_{m}+1\right) j}$ $\prod_{0 \leq i \leq m-1, i \neq j}\left(\zeta_{m}^{i}-\zeta_{m}^{j}\right)=\frac{1}{m} \zeta_{m}^{\left(o_{m}+1\right) j} \zeta_{m}^{j(m-1)} \prod_{1 \leq i \leq m-1}\left(\zeta_{m}^{i}-1\right)=\frac{1}{m} \zeta_{m}^{o_{m} j} m=\left(\zeta_{m}^{j}\right)^{o_{m}}$. Since $\operatorname{deg}(g(y)) \leq m-1$ and $g\left(\zeta_{m}^{j}\right)=\left(\zeta_{m}^{j}\right)^{o_{m}}$ for $j=0, \ldots, m-1$, we have $g(y)=y^{o_{m}}$. Hence $y^{x}-y^{o_{m}} \in\left(f_{0}, \ldots, f_{m-1}\right) \subset\left\langle y^{m}-1\right\rangle$.

Corollary 4.39 For $m \in \mathbb{N}, a \in \mathcal{F}^{*}$, and $\mathbf{f} \in \mathbb{Z}[x]^{n}$, we have $\mathbb{Y}^{\left(x-o_{m}\right) \mathbf{f}}-a^{x-o_{m}} \in\left\langle\mathbb{Y}^{m \mathbf{f}}-a^{m}\right\rangle$.

Proof: Let $z=\frac{\mathbb{Y}^{\mathbf{f}}}{a}$ and $\mathcal{I}=\left[\mathbb{Y}^{m \mathbf{f}}-a^{m}\right]$. Then $z^{m}-1 \in \mathcal{I}$. By Lemma 4.38, $z^{x-o_{m}}-1 \in$ $\left\langle z^{m}-1\right\rangle \subset\langle\mathcal{I}\rangle$. Then $\left(\frac{\mathbb{P}^{\mathbf{f}}}{a}\right)^{x-o_{m}}-1 \in\langle\mathcal{I}\rangle$ or $\mathbb{Y}^{\left(x-o_{m}\right) \mathbf{f}}-a^{x-o_{m}} \in\langle\mathcal{I}\rangle$.

The following example shows that the generators of a well-mixed or perfect ideal depend on the difference field $\mathcal{F}$.

Example 4.40 Let $\mathcal{F}=\mathbb{Q}(\sqrt{-3})$ and $p=y_{1}^{3}-1$. Following Lemma 4.35, if $\sigma(\sqrt{-3})=$ $\sqrt{-3}$, we have $o_{3}=1$ and $\langle p\rangle=\{p\}=\left[p, y_{1}^{x}-y_{1}\right]$. If $\sigma(\sqrt{-3})=-\sqrt{-3}$, we have $o_{3}=2$ and $\langle p\rangle=\{p\}=\left[p, y_{1}^{x}-y_{1}^{2}\right]$. If $\mathcal{F}=\mathbb{Q}$ then $\{p\}=\left[y_{1}-1\right] \cap\left[y_{1}^{2}+y_{1}+1, y_{1}^{x}-y_{1}\right] \cap\left[y_{1}^{2}+y_{1}+1, y_{1}^{x}-y_{1}^{2}\right]$ does not has a set of simple generators.

Motivated by Corollary 4.39, we have the following definition.

Definition 4.41 If a $\mathbb{Z}[x]$-lattice L satisfies

$$
m \mathbf{f} \in L \Rightarrow\left(x-o_{m}\right) \mathbf{f} \in L
$$

where $m \in \mathbb{N}, \mathbf{f} \in \mathbb{Z}[x]^{n}$, and $o_{m}$ is defined in Lemma 4.35, then it is called M-saturated. For any $\mathbb{Z}[x]$-lattice $L$, the smallest $\mathrm{M}$-saturated $\mathbb{Z}[x]$-lattice containing $L$ is called the $\mathrm{M}$ saturation of $L$ and is denoted by $\operatorname{sat}_{M}(L)$.

The following result gives an effective version for condition (23).

Lemma 4.42 $A \mathbb{Z}[x]^{n}$-lattice $L$ is $M$-saturated if and only if the following condition is true: Let $L_{1}=\operatorname{sat}_{\mathbb{Z}}(L)=\left(\mathbf{g}_{1}, \ldots, \mathbf{g}_{s}\right)$ such that $m_{i} \mathbf{g}_{i} \in L$ for $m_{i} \in \mathbb{N}$. Then $\left(x-o_{m_{i}}\right) \mathbf{g}_{i} \in L$. 
Proof: We need only to show $\left(x-o_{m_{i}}\right) \mathbf{g}_{i} \in L$ implies (23). For any $m \mathbf{f} \in L$, we have $\mathbf{f} \in L_{1}$ and hence $\mathbf{f}=\sum_{i=1}^{r} q_{i} \mathbf{g}_{i}$, where $q_{i} \in \mathbb{Z}[x]$. Let $t=\operatorname{lcm}\left(m, m_{1}, \ldots, m_{s}\right)$. By Corollary 4.37. we have $o_{t}=o_{m_{i}}+c_{i} m_{i}$, where $c_{i} \in \mathbb{Z}$. Then $\left(x-o_{t}\right) \mathbf{f}=\sum_{i=1}^{r} q_{i}\left(x-o_{t}\right) \mathbf{g}_{i}=$ $\sum_{i=1}^{r} q_{i}\left(x-o_{m_{i}}\right) \mathbf{g}_{i}-\sum_{i=1}^{r} q_{i} c_{i} m_{i} \mathbf{g}_{i} \in L$. By Corollary 4.37, $o_{t}=o_{m}+c m$, where $c \in \mathbb{Z}$. Then $\left(x-o_{m}\right) \mathbf{f}=\left(x-o_{t}\right) \mathbf{f}+c m \mathbf{f} \in L$.

We now give a criterion for a Laurent binomial $\sigma$-ideal to be well-mixed in terms of its support lattice.

Theorem 4.43 Let $\rho$ be a partial character and $\mathcal{F}$ an algebraically closed and inversive $\sigma$ field. If $\mathcal{I}(\rho)$ is well-mixed, then $L_{\rho}$ is M-saturated. Conversely, if $L_{\rho}$ is M-saturated, then either $\langle\mathcal{I}(\rho)\rangle=[1]$ or $\mathcal{I}(\rho)$ is well-mixed.

Proof: Suppose that $\mathcal{I}(\rho)$ is well-mixed. If there exists an $m \in \mathbb{N}$ such that $m \mathbf{f} \in L_{\rho}$, then by Lemma 4.17, there exists a $c \in \mathcal{F}^{*}$ such that $\mathbb{Y}^{m \mathbf{f}}-c \in \mathcal{I}(\rho)$. Since $\mathcal{F}$ is algebraically closed, there exists an $a \in \mathcal{F}^{*}$ such that $c=a^{m}$. Then, $\mathbb{Y}^{m \mathbf{f}}-a^{m} \in \mathcal{I}(\rho)$. Since $\mathcal{I}(\rho)$ is well-mixed, by Corollary 4.39, $\mathbb{Y}^{\left(x-o_{m}\right) \mathbf{f}}-a^{x-o_{m}} \in \mathcal{I}(\rho)$, and by Lemma 4.17 again, $\left(x-o_{m}\right) \mathbf{f} \in L_{\rho}$ follows and $L_{\rho}$ is M-saturated.

Conversely, let $L_{\rho}$ be M-saturated. If $L_{\rho}$ is $\mathbb{Z}$-saturated, then by Theorem 4.23, $\mathcal{I}(\rho)$ is prime and hence well-mixed by Lemma 4.31. Otherwise, there exists an $m_{1} \in \mathbb{N}$, and $\mathbf{f} \in \mathbb{Z}[x]^{n}$ such that $\mathbf{f} \notin L_{\rho}$ and $m_{1} \mathbf{f} \in L_{\rho}$. By Lemma 4.17, there exists an $a \in \mathcal{F}^{*}$ such that $\mathbb{Y}^{m_{1} \mathbf{f}}-a^{m_{1}} \in \mathcal{I}(\rho)$. We claim that either $\langle\mathcal{I}(\rho)\rangle=[1]$ or

$$
\mathcal{I}(\rho)=\cap_{l_{1}=0}^{m_{1}-1} \mathcal{I}_{l_{1}}
$$

where $\mathcal{I}_{l_{1}}=\left[\mathcal{I}(\rho), \mathbb{Y}^{\mathbf{f}}-a \zeta_{m_{1}}^{l_{1}}\right]$ and $\zeta_{m_{1}}=e^{\frac{2 \pi \mathrm{i}}{m_{1}}}$. By (23), $\left(x-o_{m_{1}}\right) \mathbf{f} \in L_{\rho}$. By Lemma 4.17 there exists a $b \in \mathcal{F}^{*}$ such that $\mathbb{Y}^{\left(x-o_{m_{1}}\right) \mathbf{f}}-b \in \mathcal{I}(\rho)$. Since $\mathbb{Y}^{m_{1} \mathbf{f}}-a^{m_{1}} \in \mathcal{I}(\rho)$, by Corollary 4.36, we have $\mathbb{Y}^{\left(x-o_{m_{1}}\right) \mathbf{f}}-a^{x-o_{m_{1}}} \in\left[\mathbb{Y}^{\mathbf{f}}-a \zeta_{m_{1}}^{l_{1}}\right]$ for any $l_{1}$. Then $b-a^{x-o_{m_{1}}}=$ $\mathbb{Y}^{\left(x-o_{m_{1}}\right) \mathbf{f}}-a^{x-o_{m_{1}}}-\left(\mathbb{Y}^{\left(x-o_{m_{1}}\right) \mathbf{f}}-b\right) \in \mathcal{I}_{l_{1}}$ for any $l_{1}$. If $b \neq a^{x-o_{m_{1}}}, \mathcal{I}_{l_{1}}=[1]$ for all $l_{1}$, and hence $1 \in \cap_{l_{1}=0}^{m_{1}-1} \mathcal{I}_{l_{1}} \subset\langle\mathcal{I}(\rho)\rangle$ by Lemma 4.38 and $\langle\mathcal{I}(\rho)\rangle=[1]$ follows. Now suppose $b=a^{x-o_{m_{1}}}$ or $a^{x}=b a^{o_{m_{1}}}$. To prove (24), it suffices to show $\cap_{l_{1}=0}^{m_{1}-1} \mathcal{I}_{l_{1}} \subset \mathcal{I}(\rho)$. Let $f \in \cap_{l_{1}=0}^{m_{1}-1} \mathcal{I}_{l_{1}}$. From $f \in \mathcal{I}_{l_{1}}$, we have $f=f_{l_{1}}+\sum_{j=0}^{s} p_{j} \sigma^{j}\left(\mathbb{Y}^{\mathbf{f}}-a \zeta_{m_{1}}^{l_{1}}\right)$, where $f_{l_{1}} \in \mathcal{I}(\rho)$. By Lemma 4.35, $\sigma\left(\zeta_{m_{1}}\right)=\zeta_{m_{1}}^{o_{m_{1}}}$. We thus have

$$
\begin{aligned}
\sigma\left(\mathbb{Y}^{\mathbf{f}}-a \zeta_{m_{1}}^{l_{1}}\right) & =\mathbb{Y}^{x \mathbf{f}}-b \mathbb{Y}^{o_{m_{1}}} \mathbf{f}+b \mathbb{Y}^{o_{m_{1}} \mathbf{f}}-\sigma\left(a \zeta_{m_{1}}^{l_{1}}\right) \\
& =\mathbb{Y}^{o_{m_{1}}} \mathbf{f}\left(\mathbb{Y}^{\left(x-o_{m_{1}}\right) \mathbf{f}}-b\right)+b\left(\mathbb{Y}^{o_{m_{1}} \mathbf{f}}-a^{o_{m_{1}}} \zeta_{m_{1}}^{l_{1} o_{m_{1}}}\right)+\left(b a^{o_{m_{1}}}-\sigma(a)\right) \zeta_{m_{1}}^{l_{1} o_{m_{1}}}
\end{aligned}
$$

Since $\mathbb{Y}^{\left(x-o_{m_{1}}\right) \mathbf{f}}-b \in \mathcal{I}(\rho)$ and $b a^{o_{m_{1}}}-\sigma(a)=b a^{o_{m_{1}}}-a^{x}=0$, we have $\sigma\left(\mathbb{Y}^{\mathbf{f}}-a \zeta_{m_{1}}^{l_{1}}\right)=$ $g_{l_{1}}+q_{l_{1}}\left(\mathbb{Y}^{\mathbf{f}}-a \zeta_{m_{1}}^{l_{1}}\right)$, where $g_{l_{1}} \in \mathcal{I}(\rho)$. Using the above equation repeatedly, we have $f=h_{l_{1}}+p_{l_{1}}\left(\mathbb{Y}^{\mathbf{f}}-a \zeta_{m_{1}}^{l_{1}}\right)$, where $h_{l_{1}} \in \mathcal{I}(\rho)$. Then, $f^{m_{1}}=\prod_{l_{1}=0}^{m_{1}-1}\left(h_{l_{1}}+p_{l_{1}}\left(\mathbb{Y}^{\mathbf{f}}-a \zeta_{m_{1}}^{l_{1}}\right)\right)=$ $s+\prod_{l_{1}=0}^{m_{1}-1} p_{l_{1}}\left(\mathbb{Y}^{\mathbf{f}}-a \zeta_{m_{1}}^{l_{1}}\right)=s+\left(\mathbb{Y}^{m_{1} \mathbf{f}}-a^{m_{1}}\right) \prod_{l_{1}=0}^{m_{1}-1} p_{l_{1}} \in \mathcal{I}(\rho)$, where $s$ is in $\mathcal{I}(\rho)$. By Corollary 4.13, we have $f \in \mathcal{I}(\rho)$. The claim is proved.

The support lattice for any of $\left[\mathcal{I}_{l_{1}}\right]$ is $L_{1}=\left(L_{\rho}, \mathbf{f}\right)$. Similar to the proof of Theorem 4.26, we can show that $\mathcal{I}(\rho) \varsubsetneqq \mathcal{I}_{l_{1}}$ and $L_{\rho} \varsubsetneqq L_{1}$. If $L_{1}$ is not $\mathbb{Z}$-saturated, there exists a $k>1$ and 
$\mathbf{g} \in \mathbb{Z}[x]^{n}$ such that $\mathbf{g} \notin \mathbb{L}_{1}$ and $k \mathbf{g} \in L_{1}$. Let $m_{2}=k m_{1}$. We have $m_{2} \mathbf{g}=k m_{1} \mathbf{g} \in L_{\rho}$ and there exists a $c \in \mathcal{F}^{*}$ such that $\mathbb{Y}^{m_{2}} \mathbf{g}-c^{m_{2}} \in \mathcal{I}(\rho)$. Hence, $\left(x-o_{m_{2}}\right) \mathbf{g} \in L_{\rho} \subset L_{1}$ and there exists a $d \in \mathcal{F}^{*}$, such that $\mathbb{Y}^{\left(x-o_{m_{2}}\right)} \mathbf{g}-d \in I(\rho)$. Let $L_{2}=\left(L_{1}, \mathbf{g}\right)$ and $\mathcal{I}_{l_{1}, l_{2}}=\left[\mathcal{I}_{l_{1}}, \mathbb{Y}^{\mathbf{g}}-c \zeta_{m_{2}}^{l_{2}}\right]$, $l_{2}=0, \ldots, m_{2}-1$. Then $L_{1} \varsubsetneqq L_{2}$ and $L_{2}$ is the support lattice for all $\mathcal{I}_{l_{1}, l_{2}}$ provided $\mathcal{I}_{l_{1}, l_{2}} \neq[1]$. Similar to the above, it can be shown that $d-c^{x-o_{m_{2}}} \in \mathcal{I}_{l_{1}, l_{2}}$ for any $l_{1}, l_{2}$. If $d-c^{x-o_{m_{2}}} \neq 0$, then $\mathcal{I}_{l_{1}, l_{2}}=[1]$ for any $l_{1}, l_{2}$ and $\left\langle\mathcal{I}_{l_{1}}\right\rangle=[1]$ by Lemma 4.38. Since Laurent binomial $\sigma$-ideals are radical, $\langle\mathcal{I}(\rho)\rangle=\cap_{l_{1}=0}^{m_{1}-1}\left\langle\mathcal{I}_{l_{1}}\right\rangle=[1]$ by Lemma 4.34 and (24). If $d-c^{x-o_{m_{2}}}=0$, it can be similarly proved that $\mathcal{I}_{l_{1}}=\cap_{l_{2}=0}^{m_{2}-1} \mathcal{I}_{l_{1}, l_{2}}$ for any $l_{1}$. As a consequence, we have either $\langle I(\rho)\rangle=[1]$ or $\mathcal{I}(\rho)=\cap_{l_{1}=0}^{m_{1}-1} \mathcal{I}_{l_{1}}=\cap_{l_{1}=0}^{m_{1}-1} \cap_{l_{2}=0}^{m_{2}-1} \mathcal{I}_{l_{1}, l_{2}}$.

Repeating the process, we have either $\langle I(\rho)\rangle=[1]$ or

$$
\mathcal{I}(\rho)=\cap_{l_{1}=0}^{m_{1}-1} \mathcal{I}_{l_{1}}=\cdots=\cap_{l_{1}=0}^{m_{1}-1} \cdots \cap_{l_{t}=0}^{m_{t}-1} \mathcal{I}_{l_{1}, \ldots, l_{t}}
$$

where $L_{\rho} \varsubsetneqq L_{1} \varsubsetneqq \cdots \varsubsetneqq L_{t} \subset \operatorname{sat}_{\mathbb{Z}}\left(L_{\rho}\right)$. Since $\mathbb{Z}[x]^{n}$ is Notherian, the procedure will terminate and $L_{t}$ is $\mathbb{Z}$-saturated. Since each $\mathcal{I}_{l_{1}, \ldots, l_{t}}$ is either [1] or a prime $\sigma$-ideal, and hence either $\langle\mathcal{I}(\rho)\rangle=[1]$ or $\mathcal{I}(\rho)$ is well-mixed by Lemma 4.31 .

The following example shows that $\langle\mathcal{I}(\rho)\rangle=[1]$ can indeed happen in Theorem 4.43 .

Example 4.44 Let $\mathcal{I}=[\mathcal{A}]$, where $\mathcal{A}=\left\{y_{1}^{2}+1, y_{1}^{x}-y_{1}, y_{2}^{2}+1, y_{2}^{x}+y_{2}\right\}$ is a $\sigma$-chain. Notice that the support lattice of $\mathcal{I}$ is $M$-saturated. We have $y_{2}^{2}-y_{1}^{2}=y_{2}^{2}+1-\left(y_{1}^{2}+1\right) \in \mathcal{I}$. Then by Corollary 4.39, $y_{1} y_{2}^{x}-y_{1}^{x} y_{2} \in\langle\mathcal{I}\rangle$. From $y_{1}^{x}-y_{1}, y_{2}^{x}+y_{2} \in \mathcal{I}$, we have $y_{1} y_{2} \in\langle\mathcal{I}\rangle$ and hence $1 \in\langle\mathcal{I}\rangle$.

The following example shows that Theorem 4.51 is not valid if condition (23) is replaced by $m \mathbf{f} \in L_{\rho} \Rightarrow(x-k) \mathbf{f} \in L_{\rho}$ for some $k \in \mathbb{N}$.

Example 4.45 Let $\mathcal{F}=\mathbb{Q}(\sqrt{-3})$ and $\sigma(\sqrt{-3})=-\sqrt{-3}$. Then $o_{3}=2$. Let $p_{1}=y_{1}^{3}-1, p_{2}=$ $y_{1}^{x-1}-1$. Then $p_{1}, p_{2}$ consist of a Laurent regular and coherent $\sigma$-chain, and by Theorem 4.14. $\mathcal{I}=\operatorname{sat}\left(p_{1}, p_{2}\right)=\left[p_{1}, p_{2}\right]$ is proper and with support lattice $([3],[x-1])$. By Example 4.40, $y_{1}^{x-2}-1 \in\langle\mathcal{I}\rangle$ and then $y_{1}-1=-y_{1}^{2}\left(\left(y_{1}^{x-2}-1\right)-\left(y_{1}^{x-1}-1\right)\right) / y^{x} \in\langle\mathcal{I}\rangle$ and thus $\langle\mathcal{I}\rangle=\left[y_{1}-1\right]$.

We now prove the main result of this section.

Theorem 4.46 Let $\mathcal{F}$ be an algebraically closed and inversive $\sigma$-field and $\mathcal{I}=\mathcal{I}(\rho)$ a Laurent binomial $\sigma$-ideal. Then the well-mixed closure of $\mathcal{I}$ is either [1] or a Laurent binomial $\sigma$-ideal whose support lattice is $\operatorname{sat}_{M}\left(L_{\rho}\right)$.

Proof: Let us suppose that $\langle\mathcal{I}(\rho)\rangle \neq[1]$. If $L$ is not M-saturated, then there exists an $m \in \mathbb{N}$ and $\mathbf{f} \in \mathbb{Z}[x]^{n}$ such that $\mathbf{f} \notin L, m \mathbf{f} \in L$, and $\left(x-o_{m}\right) \mathbf{f} \notin L$. By Lemma 4.17, there exists a $c \in \mathcal{F}^{*}$ such that $\mathbb{Y}^{m \mathbf{f}}-c^{m} \in \mathcal{I}(\rho)$. Let $\mathcal{I}_{1}=\left[\mathcal{I}, \mathbb{Y}^{\left(x-o_{m}\right) \mathbf{f}}-c^{x-o_{m}}\right]$ and $L_{1}=\left(L,\left(x-o_{m}\right) \mathbf{f}\right)$. By Corollary 4.39, $\mathbb{Y}^{\left(x-o_{m}\right) \mathbf{f}}-c^{x-o_{m}} \in\langle\mathcal{I}(\rho)\rangle$. Let $L_{M}=\operatorname{sat}_{M}(L)$. Then $\mathcal{I} \varsubsetneqq \mathcal{I}_{1} \subset\langle\mathcal{I}\rangle$ and $L \varsubsetneqq L_{1} \subset L_{M}$. Repeat the procedure to construct $I_{i}$ and $L_{i}$ for $i=2, \ldots, t$ such that $\mathcal{I} \varsubsetneqq \mathcal{I}_{1} \varsubsetneqq \cdots \varsubsetneqq \mathcal{I}_{t} \subset\{\mathcal{I}\}$ and $L \varsubsetneqq L_{1} \varsubsetneqq \cdots \varsubsetneqq L_{t} \subset L_{M}$. Since $\mathbb{Z}[x]^{n}$ is Notherian, the procedure will terminate at, say $t$. Then $L_{t}=L_{M}$ is M-saturated. By Lemma 4.50, $L_{t}$ is also $x$-saturated. By Theorem 4.43, $\mathcal{I}_{t} \subset\langle\mathcal{I}\rangle$ is well-mixed and hence $\mathcal{I}_{t}=\langle\mathcal{I}\rangle$. 
By Corollary 4.27 and the proof of Theorem 4.46, we have

Corollary 4.47 A $\mathbb{Z}[x]$-lattice and its $M$-saturation have the same rank. Hence, for a Laurent binomial $\sigma$-ideal $\mathcal{I}$, either $\langle\mathcal{I}\rangle=[1]$ or $\operatorname{dim}(\langle\mathcal{I}\rangle)=\operatorname{dim}(\mathcal{I})$.

Example 4.48 Let $p=y_{2}^{2}-y_{1}^{2}$. Following the proof of Theorem 4.46, it can be shown that $\langle p\rangle=\{p\}=\left[y_{1}^{-2} y_{2}^{2}-1, y_{1}^{1-x} y_{2}^{x-1}-1\right]=\left[y_{2}^{2}-y_{1}^{2}, y_{1} y_{2}^{x}-y_{1}^{x} y_{2}\right]$.

In the rest of this section, we prove similar results for the perfect closure of Laurent binomial $\sigma$-ideals. We first give a definition.

Definition 4.49 If a $\mathbb{Z}[x]$-lattice is both $x$-saturated and $M$-saturated, then it is called $\mathrm{P}$ saturated. For any $\mathbb{Z}[x]$-lattice $L$, the smallest $\mathrm{P}$-saturated $\mathbb{Z}[x]$-lattice containing $L$ is called the $\mathrm{P}$-saturation of $L$ and is denoted by $\operatorname{sat}_{P}(L)$.

Lemma 4.50 For any $\mathbb{Z}[x]$-lattice $L$, $\operatorname{sat}_{P}(L)=\operatorname{sat}_{x}\left(\operatorname{sat}_{M}(L)\right)=\operatorname{sat}_{M}\left(\operatorname{sat}_{x}(L)\right)$.

Proof: Let $L_{1}=\operatorname{sat}_{x}\left(\operatorname{sat}_{M}(L)\right)$ and $L_{2}=\operatorname{sat}_{M}\left(\operatorname{sat}_{x}(L)\right)$. It suffices to show $L_{1}=L_{2}$. We claim that $L_{1}$ is P-saturated. Let $m \mathbf{f} \in L_{1}$ for $m \in \mathbb{N}$. Then $m x^{a} \mathbf{f} \in \operatorname{sat}_{M}(L)$ for some $a \in \mathbb{N}$, which implies $\left(x-o_{m}\right) x^{a} \mathbf{f} \in L \subset \operatorname{sat}_{x}\left(\operatorname{sat}_{M}(L)\right)=L_{1}$. Since $L_{1}$ is $x$-saturated, $\left(x-o_{m}\right) \mathbf{f} \in L_{1}$ and the claim is proved. Since $L \subset \operatorname{sat}_{M}(L), \operatorname{sat}_{x}(L) \subset \operatorname{sat}_{x}\left(\operatorname{sat}_{M}(L)\right)=L_{1}$. From the claim, $L_{1}$ is P-saturated and hence $L_{2} \subset \operatorname{sat}_{M}\left(L_{1}\right)=L_{1}$.

For the other direction, we claim that $L_{2}$ is $x$-saturated. Let $x \mathbf{f} \in \operatorname{sat}_{M}\left(\operatorname{sat}_{x}(L)\right) \subset$ $\operatorname{sat}_{\mathbb{Z}}\left(\operatorname{sat}_{x}(L)\right)$. Then there exists an $m \in \mathbb{N}$, such that $m \mathbf{f} \in \operatorname{sat}_{x}(L)$ which implies $(x-$ $\left.o_{m}\right) \mathbf{f} \in \operatorname{sat}_{M}\left(\operatorname{sat}_{x}(L)\right)$ and hence $o_{m} \mathbf{f}=x \mathbf{f}-\left(x-o_{m}\right) \mathbf{f} \in \operatorname{sat}_{M}\left(\operatorname{sat}_{x}(L)\right)$ follows. By Lemma 4.35. $\operatorname{gcd}\left(o_{m}, m\right)=1$. Then $\mathbf{f} \in \operatorname{sat}_{M}\left(\operatorname{sat}_{x}(L)\right)$, and the claim is true. Since $\operatorname{sat}_{M}(L) \subset$ $\operatorname{sat}_{M}\left(\operatorname{sat}_{x}(L)\right)=L_{2}=\operatorname{sat}_{x}\left(\operatorname{sat}_{M}\left(\operatorname{sat}_{x}(L)\right)\right)$, we have $L_{1} \subset L_{2}$.

A $\sigma$-ideal $\mathcal{I}$ is perfect if and only if $\mathcal{I}$ is reflexive, radical, and well-mixed. Since a Laurent binomial $\sigma$-ideal $\mathcal{I}$ is always radical, $\mathcal{I}$ is perfect if and only if $\mathcal{I}$ is reflexive and well-mixed. Following from this observation, we can deduce the following results about perfect Laurent binomial $\sigma$-ideal ideals.

Theorem 4.51 Let $\rho$ be a partial character and $\mathcal{F}$ an algebraically closed and inversive $\sigma$ field. If $\mathcal{I}(\rho)$ is perfect, then $L_{\rho}$ is $\mathrm{P}$-saturated. Conversely, if $L_{\rho}$ is $\mathrm{P}$-saturated, then either $\{\mathcal{I}(\rho)\}=[1]$ or $\mathcal{I}(\rho)$ is perfect.

Proof: If $\mathcal{I}(\rho)$ is perfect, then it is well-mixed and reflexive. By Theorems 4.43 and Theorem 4.23, $L_{\rho}$ is M-saturated and $x$-saturated, and hence P-saturated. Conversely, if $L_{\rho}$ is P-saturated, it is M-saturated and $x$-saturated. By Theorem 4.43, either $\langle\mathcal{I}(\rho)\rangle=[1]$ or $\mathcal{I}(\rho)$ is well-mixed. If $\langle\mathcal{I}(\rho)\rangle=[1],\{\mathcal{I}(\rho)\}=[1]$. Otherwise, by Theorem 4.23, $\mathcal{I}(\rho)$ is reflexive. By Corollary 4.13, $\mathcal{I}(\rho)$ is radical. Then $\mathcal{I}(\rho)$ is perfect.

Theorem 4.52 Let $\mathcal{F}$ be an algebraically closed and inversive $\sigma$-field and $\mathcal{I}=\mathcal{I}(\rho)$ a Laurent binomial $\sigma$-ideal. Then the perfect closure of $\mathcal{I}$ is either [1] or a Laurent binomial $\sigma$-ideal whose support lattice is $\operatorname{sat}_{P}\left(L_{\rho}\right)$. 
Proof: Let $\mathcal{I}_{x}$ be the reflexive closure of $\mathcal{I}$ and $L_{x}=\operatorname{sat}_{x}\left(L_{\rho}\right)$. By Theorem 4.26, $L_{x}$ is the support lattice of $\mathcal{I}_{x}$ and $\mathcal{I}_{x}$ is reflexive. Let $\mathcal{I}_{p}$ be the well-mixed closure of $\mathcal{I}_{x}$ and $L_{p}=\operatorname{sat}_{M}\left(\operatorname{sat}_{x}\left(L_{\rho}\right)\right)$. By Theorem 4.46, $\mathcal{I}_{p}$ is either [1] or well-mixed, and in the latter case $L_{p}$ is the support lattice of $\mathcal{I}_{p}$. By Lemma 4.50, $L_{p}=\operatorname{sat}_{P}\left(L_{\rho}\right)$. Then $\mathcal{I}_{p}$ is either [1] or perfect by Theorem 4.51 .

With Theorem 4.46 and Theorem 4.51, (3) and (4) of Theorem 1.2 are proved.

\section{Binomial $\sigma$-ideal}

In this section, properties of binomial $\sigma$-ideals will be proved. First, certain results from [7] will be extended to the difference case using the theory of infinite Gröbner basis [16. Then, properties proved in Section 4 will be extended to so-called normal binomial $\sigma$-ideals.

\subsection{Basic properties of binomial $\sigma$-ideal}

A $\sigma$-binomial in $\mathbb{Y}$ is a $\sigma$-polynomial with at most two terms, that is, $a \mathbb{Y}^{\mathbf{a}}+b \mathbb{Y}^{\mathbf{b}}$ where $a, b \in \mathcal{F}$ and $\mathbf{a}, \mathbf{b} \in \mathbb{N}[x]^{n}$. For $\mathbf{f} \in \mathbb{Z}[x]^{n}$, let $\mathbf{f}^{+}, \mathbf{f}^{-} \in \mathbb{N}^{n}[x]$ denote the positive part and the negative part of $\mathbf{f}$ such that $\mathbf{f}=\mathbf{f}^{+}-\mathbf{f}^{-}$. Consider a $\sigma$-binomial $f=a \mathbb{Y}^{\mathbf{a}}+b \mathbb{Y}^{\mathbf{b}}$, where $a, b \in \mathcal{F}^{*}$. Without loss of generality, assume $\mathbf{a}>\mathbf{b}$ according to the order defined in Section 3.1. Then $f$ has the following canonical representation

$$
f=a \mathbb{Y}^{\mathbf{a}}+b \mathbb{Y}^{\mathbf{b}}=a \mathbb{Y}^{\mathbf{g}}\left(\mathbb{Y}^{\mathbf{f}+}-c \mathbb{Y}^{\mathbf{f}^{-}}\right)
$$

where $c=\frac{-b}{a}, \mathbf{f}=\mathbf{a}-\mathbf{b} \in \mathbb{Z}[x]^{n}$ is a normal vector, and $\mathbf{g}=\mathbf{a}-\mathbf{f}^{+} \in \mathbb{N}[x]$. The normal vector $\mathbf{f}$ is called the support of $f$. Note that $\operatorname{gcd}\left(\mathbb{Y}^{\mathbf{f}+}, \mathbb{Y}^{\mathbf{f}^{-}}\right)=1$.

A $\sigma$-ideal in $\mathcal{F}\{\mathbb{Y}\}$ is called binomial if it is generated by, possibly infinitely many, $\sigma$ binomials.

In the following, $\mathcal{F}\{\mathbb{Y}\}$ is considered as a polynomial ring in infinitely many variables $\Theta(\mathbb{Y})=\left\{y_{i}^{x^{j}}, i=1, \ldots, n ; j \geq 0\right\}$ and denoted by $S=\mathcal{F}[\Theta(\mathbb{Y})]$. A theory of Gröbner basis in the case of infinitely many variables is developed in [16] and will be used in this section. For any $m \in \mathbb{N}$, denote $\Theta^{\langle m\rangle}(\mathbb{Y})=\left\{y_{i}^{x^{j}}, i=1, \ldots, n ; j=0,1, \ldots, m\right\}$ and $S^{\langle m\rangle}=\mathcal{F}\left[\Theta{ }^{\langle m\rangle}(\mathbb{Y})\right]$.

A monomial order in $S$ is called compatible with the difference structure, if $y_{i}^{x^{k_{1}}}<y_{i}^{x^{k_{2}}}$ for $k_{1}<k_{2}$. Only compatible monomial orders are considered in this section.

Let $\mathcal{I}$ be a $\sigma$-ideal in $\mathcal{F}\{\mathbb{Y}\}$. Then $\mathcal{I}$ is an algebraic ideal in $S$. By [16], we have

Lemma 5.1 Let $\mathcal{I}$ be a binomial $\sigma$-ideal in $\mathcal{F}\{\mathbb{Y}\}$. Then for a compatible monomial order, the reduced Gröbner basis $\mathbb{G}$ of $\mathcal{I}$ exists and satisfies

$$
\mathbb{G}=\cup_{m=0}^{\infty} \mathbb{G}^{\langle m\rangle}
$$

where $\mathbb{G}^{\langle m\rangle}=\mathbb{G} \cap S^{\langle m\rangle}$ is the reduced Gröbner basis of $\mathcal{I}^{\langle m\rangle}=\mathcal{I} \cap S^{\langle m\rangle}$ in the polynomial $\operatorname{ring} S^{\langle m\rangle}$. 
Contrary to the Laurent case, a binomial $\sigma$-ideal may be infinitely generated, as shown by the following example.

Example 5.2 Let $\mathcal{I}=\left[y_{1}^{x^{i}} y_{2}^{x^{j}}-y_{1}^{x^{j}} y_{2}^{x^{i}}: 0 \leq i<j \in \mathbb{N}\right]$. It is clear that $\mathcal{I}$ does not have a finite set of generators and hence a finite Gröbner basis. The Gröbner basis of

$$
\mathcal{I}^{\langle m\rangle}=\mathcal{I} \cap \mathbb{Q}\left[y_{1}, y_{2} ; y_{1}^{x}, y_{2}^{x} ; \ldots ; y_{1}^{x^{m}}, y_{2}^{x^{m}}\right]
$$

is $\left\{y_{1}^{x^{i}} y_{2}^{x^{j}}-y_{1}^{x^{i}} y_{2}^{x^{i}}: 0 \leq i<j \leq m\right\}$ with a monomial order satisfying $y_{1}<y_{2}<y_{1}^{x}<y_{2}^{x}<$ $\cdots<y_{1}^{x^{m}}<y_{2}^{x^{m}}$. Then $\left\{y_{1}^{x^{i}} y_{2}^{x^{j}}-y_{1}^{x^{j}} y_{2}^{x^{i}}: 0 \leq i<j \in \mathbb{N}\right\}$ is an infinite reduced Gröbner basis for $\mathcal{I}$ in the sense of [16] when $y_{1}^{x^{m}}$ and $y_{2}^{x^{m}}$ are treated as independent variables.

Remark 5.3 The above concept of Gröbner basis does not consider the difference structure. The concept may be refined by introducing the reduced $\sigma$-Gröbner basis [3]. A $\sigma$-monomial $M_{1}$ is called reduced w.r.t. another $\sigma$-monomial $M_{2}$ if there do not exist a $\sigma$-monomial $M_{0}$ and a $k \in \mathbb{N}$ such that $M_{1}=M_{0} M_{1}^{x^{k}}$. Then the reduced $\sigma$-Gröbner basis of $\mathcal{I}$ in Example 5.2 is $\left\{y_{1} y_{2}^{x^{i}}-y_{1}^{x^{i}} y_{2}: i \in \mathbb{Z}_{\geq 1}\right\}$ which is still infinite. Since the purpose of Gröbner basis in this paper is theoretic and not computational, we will use the version of infinite Gröbner basis in the sense of [16].

With Lemma 5.1, a large portion of the properties for algebraic binomial ideals proved by Eisenbud and Sturmfels in [7] can be extended to the difference case. The proofs follow the same pattern: to prove a property for $\mathcal{I}$, we first show that the property is valid for $\mathcal{I}$ if and only if it is valid for all $\mathcal{I}^{\langle m\rangle}$, and then the corresponding statement from [7] will be used to show that the property is indeed valid for $\mathcal{I}^{\langle m\rangle}$. We will illustrate the procedure in the following corollary. For other results, we omit the proofs.

Corollary 5.4 Let $\mathcal{I} \subset \mathcal{F}\{\mathbb{Y}\}$ be a binomial $\sigma$-ideal. Then the Gröbner basis $\mathbb{G}$ of $\mathcal{I}$ consists of $\sigma$-binomials and the normal form of any $\sigma$-term modulo $\mathbb{G}$ is again a $\sigma$-term.

Proof: By a $\sigma$-term, we mean the multiplication of an element from $\mathcal{F}^{*}$ and a $\sigma$-monomial. By (26), it suffices to show that corollary is valid for all $\mathbb{G}^{\langle m\rangle}$, that is, the Gröbner basis $\mathbb{G}^{\langle m\rangle}$ of $\mathcal{I}^{\langle m\rangle}$ consists of binomials and the normal form of any term modulo $\mathbb{G}^{\langle m\rangle}$ is again a term. Since $\mathbb{G}^{\langle m\rangle}$ is the Gröbner basis of $\mathcal{I}^{\langle m\rangle}=\mathcal{I} \cap S^{\langle m\rangle}$ and $\mathcal{I}^{\langle m\rangle}$ is a binomial ideal in a polynomial ring with finitely many variables, the corollary follows from Proposition 1.1 in 7].

Corollary 5.5 A $\sigma$-ideal $\mathcal{I}$ is binomial if and only if the reduced Gröbner basis for $\mathcal{I}$ consists of $\sigma$-binomials.

Corollary 5.6 If $\mathcal{I}$ is a binomial $\sigma$-ideal, then the elimination ideal $\mathcal{I} \cap \mathcal{F}\left\{y_{1}, y_{2}, \ldots, y_{r}\right\}$ is binomial for every $r \leq n$.

Now we consider the intersection of two ideals. The following lemma can be proved similar to its algebraic counterpart. 
Lemma 5.7 If $\mathcal{I}$ and $\mathcal{J}$ are binomial $\sigma$-ideals in $\mathcal{F}\{\mathbb{Y}\}$ then we have $\mathcal{I} \cap \mathcal{J}=[t \mathcal{I}+(1-$ $t) \mathcal{J}] \cap \mathcal{F}\{\mathbb{Y}\}$ where $t$ is a new $\sigma$-indeterminate.

The intersection of binomial $\sigma$-ideals is not binomial in general, but from Lemma 5.7 and [7] we have

Corollary 5.8 If $\mathcal{I}$ and $\mathcal{I}^{\prime}$ are binomial $\sigma$-ideals and $\mathcal{J}_{1}, \ldots, \mathcal{J}_{s}$ are $\sigma$-ideals generated by $\sigma$-monomials, then $\left[\mathcal{I}+\mathcal{I}^{\prime}\right] \cap\left[\mathcal{I}+\mathcal{J}_{1}\right] \cap \ldots \cap\left[\mathcal{I}+\mathcal{J}_{s}\right]$ is binomial.

Corollary 5.9 Let $\mathcal{I}$ be a binomial $\sigma$-ideal and let $\mathcal{J}_{1}, \ldots, \mathcal{J}_{s}$ be monomial $\sigma$-ideals.

(a) The intersection $\left[\mathcal{I}+\mathcal{J}_{1}\right] \cap \cdots \cap\left[\mathcal{I}+\mathcal{J}_{s}\right]$ is generated by $\sigma$-monomials modulo $\mathcal{I}$.

(b) Any $\sigma$-monomial in the sum $\mathcal{I}+\mathcal{J}_{1}+\cdots+\mathcal{J}_{\text {s }}$ lies in one of the $\sigma$-ideals $\mathcal{I}+\mathcal{J}_{i}$.

Corollary 5.10 If $\mathcal{I}$ is a binomial $\sigma$-ideal, then for any $\sigma$-monomial $M$, the $\sigma$-ideal quotients $[\mathcal{I}: M]$ and $\left[\mathcal{I}: M^{\infty}\right]$ are binomial.

Corollary 5.11 Let $\mathcal{I}$ be a binomial $\sigma$-ideal and $\mathcal{J}$ a monomial $\sigma$-ideal. If $f \in \mathcal{I}+\mathcal{J}$ and $g$ is the sum of those terms of $f$ that are not individually contained in $\mathcal{I}+\mathcal{J}$, then $g \in \mathcal{J}$.

Finally, from [7, Theorem 3.1], we have

Theorem 5.12 If $\mathcal{I}$ is a binomial $\sigma$-ideal, then the radical of $\mathcal{I}$ is binomial.

We now consider whether a $\sigma$-ideal is reflexive. We first give a criterion for reflexiveness.

Lemma 5.13 $A$-ideal $\mathcal{I}$ is reflexive if and only if $b^{x} \in \mathcal{I} \Rightarrow b \in \mathcal{I}$ for any $\sigma$-binomial $b \in \mathcal{F}\{\mathbb{Y}\}$.

Proof: We need only to prove one side of the statement, that is, if $b^{x} \in \mathcal{I} \Rightarrow b \in \mathcal{I}$ for any $\sigma$-binomial $b$ then $\mathcal{I}$ is reflexive. Let $p$ be a $\sigma$-polynomial such that $p^{x} \in \mathcal{I}$. Then, there exists an $m \in \mathbb{N}$ such that $p^{x} \in \mathcal{I}^{\langle m\rangle}=\mathcal{I} \cap S^{\langle m\rangle}$. Let $\mathbb{G}$ be the (finite) reduced Gröbner basis of $\mathcal{I}^{\langle m\rangle}$ in $S^{\langle m\rangle}$ under the variable order $y_{i}^{x^{j}}<y_{k}$ for any $i, j, k$. By Proposition 1.1 in [7, $\mathbb{G}$ consists of binomials. $p^{x}$ can be reduced to zero by $\mathbb{G}$. Due to the chosen variable order, we have $p^{x}=\sum_{i} e_{i}^{x} g_{i}^{x}$, where $e_{i}^{x} \in S^{\langle m\rangle}$ and $g_{i}^{x}$ is a $\sigma$-binomial in $S^{\langle m\rangle}$. Since $g_{i}^{x}$ are $\sigma$-binomials in $\mathcal{I}$, we have $g_{i} \in \mathcal{I}$. Then, $p=\sum_{i} e_{i} g_{i} \in \mathcal{I}$ and $\mathcal{I}$ is reflexive.

Theorem 5.14 If $\mathcal{I}$ is a binomial $\sigma$-ideal, then the reflexive closure of $\mathcal{I}$ is binomial.

Proof: Let $\mathcal{I}_{1}$ be the $\sigma$-ideal generated by the $\sigma$-binomials $p$ such that $p^{x^{k}} \in \mathcal{I}$ for some $k \in \mathbb{N}$. We claim that $\mathcal{I}_{1}$ is the reflexive closure of $\mathcal{I}$ and it suffices to show that $\mathcal{I}_{1}$ is reflexive. Let $p$ be a $\sigma$-binomial such that $p^{x} \in \mathcal{I}_{1}$. Then for some $k \in \mathbb{N},\left(p^{x}\right)^{x^{k}}=p^{x+1} \in \mathcal{I}$. Thus $p \in \mathcal{I}_{1}$ and $\mathcal{I}_{1}$ is reflexive by Lemma 5.13 . 


\subsection{Normal binomial $\sigma$-ideal}

In this section, most of the results about Laurent binomial $\sigma$-ideals proved in Section 4 will be extended to normal binomial $\sigma$-ideals.

Let $\mathrm{m}$ be the multiplicative set generated by $y_{i}^{x^{j}}$ for $i=1, \ldots, n, j \in \mathbb{N}$. A $\sigma$-ideal $\mathcal{I}$ is called normal if for any $M \in \mathrm{m}$ and $p \in \mathcal{F}\{\mathbb{Y}\}, M p \in \mathcal{I}$ implies $p \in \mathcal{I}$. For any $\sigma$-ideal $\mathcal{I}$,

$$
\mathcal{I}: \mathrm{m}=\{f \in \mathcal{F}\{\mathbb{Y}\} \mid \exists M \in \mathrm{m} \text { s.t. } M f \in \mathcal{I}\}
$$

is a normal $\sigma$-ideal. For any $\sigma$-ideal $\mathcal{I}$ in $\mathcal{F}\{\mathbb{Y}\}$, it is easy to check that

$$
\mathcal{F}\left\{\mathbb{Y}^{ \pm}\right\} \mathcal{I} \cap \mathcal{F}\{\mathbb{Y}\}=\mathcal{I}: \mathrm{m}
$$

We first prove a property for general normal $\sigma$-ideals.

Lemma 5.15 Let $\mathcal{I}$ be a normal $\sigma$-ideal in $\mathcal{F}\{\mathbb{Y}\}$. Then $\mathcal{I}$ is reflexive (radical, perfect, prime) if and only if $\mathcal{F}\left\{\mathbb{Y}^{ \pm}\right\} \mathcal{I}$ is reflexive (radical, perfect, prime) in $\mathcal{F}\left\{\mathbb{Y}^{ \pm}\right\}$.

Proof: Let $\overline{\mathcal{I}}=\mathcal{F}\left\{\mathbb{Y}^{ \pm}\right\} \mathcal{I}$ be a Laurent $\sigma$-ideal. Since $\mathcal{I}$ is normal, from (27) we have $\overline{\mathcal{I}} \cap \mathcal{F}\{\mathbb{Y}\}=\mathcal{I}$. If $\overline{\mathcal{I}}$ is reflexive, it is clear that $\mathcal{I}$ is reflexive. For the other direction, if $f^{x} \in \overline{\mathcal{I}}$, then by clearing denominators of $f^{x}$, there exists a $\sigma$-monomial $M^{x}$ in $\mathbb{Y}$ such that $M^{x} f^{x} \in \overline{\mathcal{I}} \cap \mathcal{F}\{\mathbb{Y}\}=\mathcal{I}$. Since $\mathcal{I}$ is reflexive, $M f \in \mathcal{I}$ and hence $f \in \overline{\mathcal{I}}$, that is, $\overline{\mathcal{I}}$ is reflexive. The results about radical and perfect $\sigma$-ideals can be proved similarly.

We now show that $\mathcal{I}$ is prime if and only if $\overline{\mathcal{I}}$ is prime. If $\overline{\mathcal{I}}$ is prime, it is clear that $\mathcal{I}$ is also prime. For the other side, let $f g \in \overline{\mathcal{I}}$. Then there exist $\sigma$-monomials $N_{1}, N_{2}$ such that $N_{1} f \in \mathcal{F}\{\mathbb{Y}\}, N_{2} g \in \mathcal{F}\{\mathbb{Y}\}$, and hence $N_{1} f N_{2} g \in \mathcal{I}$. Since $\mathcal{I}$ is prime, $N_{1} f$ or $N_{2} g$ is in $\mathcal{I}$ that is $f$ or $g$ is in $\overline{\mathcal{I}}$.

We now consider normal binomial $\sigma$-ideals. Given a partial character $\rho$ on $\mathbb{Z}[x]^{n}$, we define the following binomial $\sigma$-ideal in $\mathcal{F}\{\mathbb{Y}\}$

$$
\mathcal{I}^{+}(\rho)=\left[\mathbb{Y}^{\mathbf{f}^{+}}-\rho(\mathbf{f}) \mathbb{Y}^{\mathbf{f}^{-}}: \mathbf{f} \in L_{\rho}\right]
$$

We will show that any normal binomial $\sigma$-ideal can be written as the form (28).

Lemma 5.16 Let $\rho$ be a partial character on $\mathbb{Z}[x]^{n}$ and $\mathcal{I}(\rho)$ defined in (16). Then $\mathcal{I}^{+}(\rho)=$ $\mathcal{I}(\rho) \cap \mathcal{F}\{\mathbb{Y}\}$. As a consequence, $\mathcal{I}^{+}(\rho)$ is proper and normal.

Proof: It is clear that $\mathcal{I}^{+}(\rho) \subset \mathcal{I}(\rho) \cap \mathcal{F}\{\mathbb{Y}\}$. If $f \in \mathcal{I}(\rho) \cap \mathcal{F}\{\mathbb{Y}\}$, then $f=\sum_{i=1}^{s} f_{i} M_{i}\left(\mathbb{Y}^{\mathbf{f}_{i}}\right.$ $\left.\rho\left(\mathbf{f}_{i}\right)\right)$ where $f_{i} \in \mathcal{F}, \mathbf{f}_{i} \in L_{\rho}$, and $M_{i}$ are Laurent $\sigma$-monomials in $\mathbb{Y}$. There exists a $\sigma$ monomial $M$ in $\mathbb{Y}$ such that

$$
M f=\sum_{i=1}^{s} f_{i} N_{i}\left(\mathbb{Y}^{\mathbf{f}_{i}^{+}}-\rho\left(\mathbf{f}_{i}\right) \mathbb{Y}^{\mathbf{f}_{i}^{-}}\right) \in \mathcal{I}^{+}(\rho),
$$

where $N_{i}$ is a $\sigma$-monomial in $\mathbb{Y}$. We will prove $f \in \mathcal{I}^{+}(\rho)$ from the above equation. Without loss of generality, we may assume that $M=y_{c}^{x^{o}}$ for some $c$ and $o \in \mathbb{N}$. Note that (29) is 
an algebraic identity in $y_{i}^{x^{k}}, i=1, \ldots, n, k \in \mathbb{N}$. If $N_{i}$ contains $y_{c}^{x^{o}}$ as a factor, we move $F_{i}=f_{i} N_{i}\left(\mathbb{Y}^{\mathbf{f}_{i}^{+}}-\rho\left(\mathbf{f}_{i}\right) \mathbb{Y}^{\mathbf{f}_{i}^{-}}\right)$to the left hand side of (29) and let $f_{1}=f-F_{i} / y_{c}^{x^{o}}$. Then $f \in \mathcal{I}^{+}(\rho)$ if and only if $f_{1} \in \mathcal{I}^{+}(\rho)$. Repeat the above procedure until no $N_{i}$ contains $y_{c}^{x^{o}}$ as a factor.

If $s=0$ in (29), then $f=0$ and the lemma is proved. Since $\operatorname{gcd}\left(\mathbb{Y}^{\mathbf{f}_{i}^{+}}, \mathbb{Y}^{\mathbf{f}_{i}^{-}}\right)=1, y_{c}^{x^{o}}$ cannot be a factor of both $\mathbb{Y}^{\mathbf{f}_{i}^{+}}$and $\mathbb{Y}^{\mathbf{f}_{i}}$. Let $\mathbb{Y}^{\mathbf{f}_{i}^{+}}$be the largest $\sigma$-monomial in (29) not containing $y_{c}^{x^{o}}$ under a given $\sigma$-monomial total order. If $\mathbb{Y}^{\mathbf{f}_{i}^{-}}$is the largest $\sigma$-monomial in (29) not containing $y_{c}^{x^{\circ}}$, the proving process is similar. There must exists another $\sigma$ binomial $f_{j} N_{j}\left(\mathbb{Y}^{\mathbf{f}_{j}^{+}}-\rho\left(\mathbf{f}_{j}\right) \mathbb{Y}^{\mathbf{f}_{j}^{-}}\right)$such that $N_{i} \mathbb{Y}_{i}^{\mathbf{f}^{+}}=N_{j} \mathbb{Y}^{\mathbf{f}_{j}^{-}}$. Let $N_{i}=\mathbb{Y}^{\mathfrak{p}_{i}}, N_{j}=\mathbb{Y}^{\mathfrak{p}_{j}}$. Then $\mathbb{Y}_{i}^{\mathbf{f}_{i}^{+}+\mathfrak{p}_{i}}=\mathbb{Y}^{\mathbf{f}_{j}^{-}+\mathfrak{p}_{j}}$ and $\mathbf{f}_{i}^{+}+\mathfrak{p}_{i}=\mathbf{f}_{j}^{-}+\mathfrak{p}_{j}$. We have $p=f_{i} N_{i}\left(\mathbb{Y}^{\mathbf{f}_{i}^{+}}-\rho\left(\mathbf{f}_{i}\right) \mathbb{Y}^{\mathbf{f}_{i}^{-}}\right)+f_{j} N_{j}\left(\mathbb{Y}^{\mathbf{f}_{j}^{+}}\right.$ $\left.\rho\left(\mathbf{f}_{j}\right) \mathbb{Y}^{\mathbf{f}_{j}^{-}}\right)=\frac{f_{i}}{\rho\left(\mathbf{f}_{j}\right)}\left(\mathbb{Y}^{\mathbf{f}_{j}^{+}+\mathfrak{p}_{j}}-\rho\left(\mathbf{f}_{i}\right) \rho\left(\mathbf{f}_{j}\right) \mathbb{Y}_{i}^{\mathbf{f}_{i}^{-}+\mathfrak{p}_{i}}\right)+\left(f_{j}-\frac{f_{i}}{\rho\left(\mathbf{f}_{j}\right)}\right) N_{j}\left(\mathbb{Y}^{\mathbf{f}_{j}^{+}}-\rho\left(\mathbf{f}_{j}\right) \mathbb{Y}^{\mathbf{f}_{j}^{-}}\right)$. Since $\mathbf{f}=$ $\mathbf{f}_{j}^{+}+\mathfrak{p}_{j}-\left(\mathbf{f}_{i}^{-}+\mathfrak{p}_{i}\right)=\mathbf{f}_{i}^{+}-\mathbf{f}_{i}^{-}+\mathbf{f}_{j}^{+}-\mathbf{f}_{j}^{-}=\mathbf{f}_{i}+\mathbf{f}_{j} \in L_{\rho}$, we have $\mathbb{Y}_{j}^{\mathbf{f}_{j}^{+}+\mathfrak{p}_{j}}-\rho\left(\mathbf{f}_{i}\right) \rho\left(\mathbf{f}_{j}\right) \mathbb{Y}_{i}^{-}+\mathfrak{p}_{i}=$ $N\left(\mathbb{Y}^{\mathbf{f}^{+}}-\rho(\mathbf{f}) \mathbb{Y}^{\mathbf{f}^{-}}\right) \in \mathcal{I}^{+}(\rho)$, where $N$ is a $\sigma$-monomial. As a consequence, $p \in \mathcal{I}^{+}(\rho)$. If $N$ contains $y_{c}^{x^{o}}$, move the term $\frac{f_{i}}{\rho\left(\mathbf{f}_{j}\right)} N\left(\mathbb{Y}^{\mathbf{f}^{+}}-\rho(\mathbf{f}) \mathbb{Y}^{\mathbf{f}^{-}}\right)$to the left hand side of (29) as we did in the first phase of the proof. After the above procedure, equation (29) is still valid. Furthermore, the number of $\sigma$-binomials in (29) does not increase, no $N_{i}$ contains $y_{c}^{x^{\circ}}$, and the largest $\sigma$-monomial $\mathbb{Y}_{i}^{\mathbf{f}_{i}^{+}}$or $\mathbb{Y}^{\mathbf{f}_{i}^{-}}$not containing $y_{c}^{x^{o}}$ becomes smaller. The above procedure will stop after a finite number of steps, which means $s=0$ in (29) and hence $y_{c}^{x^{o}} f=0$ which means the original $f$ is in $\mathcal{I}^{+}(\rho)$. Then $\mathcal{I}^{+}(\rho)=\mathcal{I}(\rho) \cap \mathcal{F}\{\mathbb{Y}\}$.

$\mathcal{I}^{+}(\rho)=\mathcal{I}(\rho) \cap \mathcal{F}\{\mathbb{Y}\}$ is proper. For otherwise $\mathcal{I}(\rho)=[1]$, contradicting to Lemma 4.16, Note that $\mathcal{I}^{+}(\rho) \mathcal{F}\left\{\mathbb{Y}^{ \pm}\right\}=\mathcal{I}(\rho)$. Then $\mathcal{I}^{+}(\rho)=\mathcal{I}(\rho) \cap \mathcal{F}\{\mathbb{Y}\}=\mathcal{I}^{+}(\rho) \mathcal{F}\left\{\mathbb{Y}^{ \pm}\right\} \cap \mathcal{F}\{\mathbb{Y}\}=$ $\mathcal{I}^{+}(\rho): \mathbb{m}$, and $\mathcal{I}^{+}(\rho)$ is normal.

Lemma 5.17 Let $\rho$ be a partial character over $\mathbb{Z}[x]^{n}$. Then $\mathbb{Y}^{\mathbf{f}^{+}}-c \mathbb{Y}^{\mathbf{f}^{-}} \in \mathcal{I}^{+}(\rho)$ if and only if $\mathbf{f} \in L_{\rho}$ and $c=\rho(\mathbf{f})$.

Proof: By Lemma 5.16, $\mathbb{Y}^{\mathbf{f}^{+}}-c \mathbb{Y}^{\mathbf{f}^{-}} \in \mathcal{I}^{+}(\rho)$ if and only if $\mathbb{Y}^{\mathbf{f}}-c \in \mathcal{I}(\rho)$ which is equivalent to $\mathbf{f} \in L_{\rho}$ and $c=\rho(\mathbf{f})$ by Lemma 4.17 ,

Lemma 5.18 If $\mathcal{I}$ is a normal binomial $\sigma$-ideal, then there exists a unique partial character $\rho$ on $\mathbb{Z}[x]^{n}$ such that $\mathcal{I}=\mathcal{I}^{+}(\rho)$ and $L_{\rho}=\left\{\mathbf{f} \in \mathbb{Z}[x]^{n} \mid \mathbb{Y}^{\mathbf{f}^{+}}-\rho(\mathbf{f}) \mathbb{Y}^{\mathbf{f}^{-}} \in \mathcal{I}\right\}$ which is called the support lattice of $\mathcal{I}$.

Proof: We have $\mathcal{I} \cdot \mathcal{F}\left\{\mathbb{Y}^{ \pm}\right\} \cap \mathcal{F}\{\mathbb{Y}\}=\mathcal{I}: \mathrm{m}$. According to Theorem 4.18, there exists a partial character $\rho$ such that $\mathcal{I} \cdot \mathcal{F}\left\{\mathbb{Y}^{ \pm}\right\}=\mathcal{I}(\rho)$. Then by Lemma 5.16, $\mathcal{I}=(\mathcal{I}: \mathrm{m})=\mathcal{I} \cdot \mathcal{F}\left\{\mathbb{Y}^{ \pm}\right\} \cap$ $\mathcal{F}\{\mathbb{Y}\}=\mathcal{I}(\rho) \cap \mathcal{F}\{\mathbb{Y}\}=\mathcal{I}^{+}(\rho)$. By Lemma 5.17, we have $L_{\rho}=\left\{\mathbf{f} \in \mathbb{Z}[x]^{n} \mid \mathbb{Y}^{\mathbf{f}^{+}}-\rho(\mathbf{f}) \mathbb{Y}^{\mathbf{f}^{-}} \in\right.$ $\left.\mathcal{I}=\mathcal{I}^{+}(\rho)\right\}$. The uniqueness of $\rho$ comes from the fact that $L_{\rho}$ is uniquely determined by $\mathcal{I}$.

By Lemmas 5.16 and 5.18, we have

Theorem 5.19 The map $\mathcal{I}(\rho) \Rightarrow \mathcal{I}^{+}(\rho)$ gives a one to one correspondence between Laurent binomial $\sigma$-ideals and normal binomial $\sigma$-ideals. 
Due to Lemma 5.16 and Theorem 5.19, most properties of Laurent binomial $\sigma$-ideals can be extended to normal binomial $\sigma$-ideals. As a consequence of Corollary 4.13, Lemma 5.15, and Lemma 5.16, we have

Corollary 5.20 A normal binomial $\sigma$-ideal is radical.

As a consequence of Theorem 4.26, Lemma 5.15, and Theorem 5.19.

Corollary 5.21 Let $\mathcal{I}=\mathcal{I}^{+}(\rho)$ be a normal binomial $\sigma$-ideal. If $\mathcal{F}$ is inversive, then the reflexive closure of $\mathcal{I}$ is also a normal binomial $\sigma$-ideal whose support lattice is the $x$-saturation of $L_{\rho}$.

Corollary 5.22 Let $\mathcal{I}=\mathcal{I}+(\rho)$ be a normal binomial $\sigma$-ideal. If $\mathcal{F}$ is algebraically closed and inversive, then

(a) $L_{\rho}$ is $\mathbb{Z}$-saturated if and only if $\mathcal{I}$ is prime;

(b) $L_{\rho}$ is $x$-saturated if and only if $\mathcal{I}$ is reflexive;

(c) $L_{\rho}$ is saturated if and only if $\mathcal{I}$ is reflexive prime.

Proof: It is easy to show that $\mathcal{I}(\rho)=\mathcal{I}^{+}(\rho) \mathcal{F}\left\{\mathbb{Y}^{ \pm}\right\}$. Then the corollary is a consequence of Theorem 4.23, Lemma 5.15, and Lemma 5.16.

For properties related with perfect $\sigma$-ideals, it becomes more complicated. Direct extension of Theorems 4.29, 4.51, and 4.52 to the normal binomial case is not correct as shown by the following example.

Example 5.23 Let $\mathcal{I}=\left[y_{1}^{x}-y_{1}, y_{2}^{2}-y_{1}^{2}, y_{2}^{x}+y_{2}\right]$ which is a normal binomial $\sigma$-ideal whose representation matrix is $L=\left[\begin{array}{ccc}x-1 & -2 & 0 \\ 0 & 2 & x-1\end{array}\right]$. Since $o_{2}=1, L$ is $P$-saturated. Also, $L_{s}=\operatorname{sat}(L)=\left[\begin{array}{cc}x-1 & -1 \\ 0 & 1\end{array}\right]$. We have $\{\mathcal{I}\}=\left\{\mathcal{I}, y_{2}-y_{1}\right\} \cap\left\{\mathcal{I}, y_{2}+y_{1}\right\}=\left[y_{1}, y_{2}\right]$. Then $\{\mathcal{I}\} \neq[1]$ and $\mathcal{I}$ is not perfect and hence Theorems 4.51 and 4.52 are not correct. Theorem 4.29 is also not correct, since the supporting lattice of the prime component of $\mathcal{I}$ is not $L_{s}$.

It can be seen that the problem is due to the occurrence of $\sigma$-monomials. For any partial character $\rho$, it can be shown that

$$
\left\{\mathcal{I}^{+}(\rho)\right\}: \mathrm{m}=\{\mathcal{I}(\rho)\} \cap \mathcal{F}\{\mathbb{Y}\}
$$

We thus have the following modifications for Theorems 4.51, 4.52, and 4.29,

Corollary 5.24 Let $\mathcal{I}=\mathcal{I}^{+}(\rho)$ be a normal binomial $\sigma$-ideal and $\mathcal{F}$ an inversive and algebraically closed $\sigma$-field. If $\mathcal{I}$ is perfect, then $L_{\rho}$ is $P$-saturated. Conversely, if $L_{\rho}$ is $P$ saturated and $x$-saturated, then either $\{\mathcal{I}\}: \mathrm{m}=[1]$ or $\mathcal{I}$ is perfect. 
Proof: If $\mathcal{I}$ is perfect, by Lemma 5.15, $\mathcal{I}(\rho)=\mathcal{I} \mathcal{F}\left\{\mathbb{Y}^{ \pm}\right\}$is also perfect. By Theorem 4.51, $L_{\rho}$ is $P$-saturated. If $L_{\rho}$ is $P$-saturated and $x$-saturated, by Theorem 4.51, either $\mathcal{I}(\rho)=[1]$ or $\mathcal{I}(\rho)$ is perfect. If $\mathcal{I}(\rho)=[1]$, by (30), $\{\mathcal{I}\}: \mathbb{m}=[1]$. If $\mathcal{I}(\rho)$ is perfect, by Lemma [5.15, $\mathcal{I}=\mathcal{I}^{+}(\rho)$ is also perfect.

Similarly, as a consequence of Theorem 4.52, Lemma 5.15, and Theorem 5.19, we have

Corollary 5.25 Let $\mathcal{I}=\mathcal{I}^{+}(\rho)$ be a normal binomial $\sigma$-ideal and $\mathcal{F}$ an inversive and algebraically closed $\sigma$-field. Then either $\{\mathcal{I}\}: \mathrm{m}=[1]$ or $\{\mathcal{I}\}: \mathrm{m}$ is a binomial $\sigma$-ideal whose support lattice is the P-saturation of $L_{\rho}$.

In the rest of this section, we give decomposition theorems for perfect binomial $\sigma$-ideals. We first consider normal binomial $\sigma$-ideals. By Corollary 5.20 and Example 4.24, a normal binomial $\sigma$-ideal is radical but may not be perfect.

Theorem 5.26 Let $\mathcal{I}=\mathcal{I}^{+}(\rho)$ be a normal binomial $\sigma$-ideal and $\mathcal{F}$ an inversive and algebraically closed $\sigma$-field. Then $\{\mathcal{I}\}: \mathrm{m}$ is either [1] or can be written as the intersection of reflexive prime binomial $\sigma$-ideals whose support lattice is the saturation lattice of $L_{\rho}$.

Proof: By Theorem 4.29, either $\{\mathcal{I}(\rho)\}=[1]$ or $\{\mathcal{I}(\rho)\}=\bigcap_{i=1}^{s} \mathcal{I}\left(\rho_{i}\right)$, where $\mathcal{I}\left(\rho_{i}\right)$ are reflexive prime $\sigma$-ideals whose support lattices are $\operatorname{sat}\left(L_{\rho}\right)$. By (30) and Lemma 5.16, either $\left\{\mathcal{I}^{+}(\rho)\right\}: \mathrm{m}=[1]$ or $\left\{\mathcal{I}^{+}(\rho)\right\}: \mathrm{m}=\{\mathcal{I}(\rho)\} \cap \mathcal{F}\{\mathbb{Y}\}=\left(\bigcap_{i=1}^{s} \mathcal{I}\left(\rho_{i}\right)\right) \cap \mathcal{F}\{\mathbb{Y}\}=\bigcap_{i=1}^{s}\left(\mathcal{I}\left(\rho_{i}\right) \cap\right.$ $\mathcal{F}\{\mathbb{Y}\})=\bigcap_{i=1}^{s} \mathcal{I}^{+}\left(\rho_{i}\right)$. By Corollary [5.22, $\mathcal{I}^{+}\left(\rho_{i}\right)$ is reflexive and prime whose support lattices are the saturation of $L_{\rho}$.

Now, consider general binomial $\sigma$-ideals.

Lemma 5.27 $\mathcal{I} \subset \mathcal{F}\{\mathbb{Y}\}$ is a reflexive prime binomial $\sigma$-ideal if and only if $\mathcal{I}=\left[y_{i_{1}}, \ldots, y_{i_{s}}\right]$ $+\mathcal{I}_{1}$, where $\left\{y_{i_{1}}, \ldots, y_{i_{s}}\right\}=\mathbb{Y} \cap \mathcal{I},\left\{z_{1}, \ldots, z_{t}\right\}=\mathbb{Y} \backslash \mathcal{I}$, and $\mathcal{I}_{1}$ is a normal binomial reflexive prime $\sigma$-ideal in $\mathcal{F}\left\{z_{1}, \ldots, z_{t}\right\}$.

Proof: If $\mathcal{I}$ is reflexive and prime, then $\left(y_{i}^{x^{j}}\right)^{d} \in \mathcal{I}$ if and only if $y_{i} \in \mathcal{I}$. Let $\mathcal{I}_{1}=\mathcal{I} \cap$ $\mathcal{F}\left\{z_{1}, \ldots, z_{t}\right\}$. Then $\mathcal{I}=\left[y_{i_{1}}, \ldots, y_{i_{s}}\right]+\mathcal{I}_{1}$. $\mathcal{I}_{1}$ is clearly reflexive and prime. We still need to show that $\mathcal{I}_{1}$ is normal. Let $N f \in \mathcal{I}_{1}$ for a $\sigma$-monomial $N$ in $\left\{z_{1}, \ldots, z_{t}\right\}$ and $f \in \mathcal{F}\left\{z_{1}, \ldots, z_{t}\right\} . N$ cannot be in $\mathcal{I}_{1}$. Otherwise, some $z_{i}$ is in $\mathcal{I}_{1}$ since $\mathcal{I}_{1}$ is reflexive and prime, which contradicts to $\left\{z_{1}, \ldots, z_{t}\right\}=\mathbb{Y} \backslash \mathcal{I}$. Therefore, $f \in \mathcal{I}_{1}$ and $\mathcal{I}_{1}$ is normal. The other direction is trivial.

The $\sigma$-ideal $\mathcal{I}$ in Lemma 5.27 is said to be quasi-normal. The following result can be proved similarly to Theorem 4.29 .

Theorem 5.28 Let $\mathcal{I}$ be a binomial $\sigma$-ideal. If $\mathcal{F}$ is algebraically closed and inversive, then the perfect $\sigma$-ideal $\{\mathcal{I}\}$ is either [1] or the intersection of quasi-normal reflexive prime binomial $\sigma$-ideals.

Proof: We prove the theorem by induction on $n$. Let $\mathcal{I}_{1}=\{\mathcal{I}\}: \mathbb{m}$. Then $\{\mathcal{I}\}=\mathcal{I}_{1} \cap$ $\cap_{i=1}^{n}\left\{\mathcal{I}, y_{i}\right\}$. It is easy to check $\mathcal{I}_{1}=\{\mathcal{I}: \mathrm{m}\}: \mathrm{m}$. Since $\mathcal{I}: \mathrm{m}$ is normal, by Theorem 5.26 , 
$\mathcal{I}_{1}$ is either [1] or intersection of normal reflexive prime $\sigma$-ideals. If $n=1$, then $\left\{\mathcal{I}, y_{i}\right\}$ must be either $\left[y_{1}\right]$ or $[1]$. Then the theorem is proved for $n=1$. Suppose the theorem is valid for $n=1, \ldots, k-1$. Still use $\{\mathcal{I}\}=\mathcal{I}_{1} \cap \cap_{i=1}^{n}\left\{\mathcal{I}, y_{i}\right\}$. Let $\mathcal{I}_{i}$ be the $\sigma$-ideal obtained by setting $y_{i}$ to 0 in $\mathcal{I}$. By the induction hypothesis, $\mathcal{I}_{i}$ can be written as intersection of quasi-normal reflexive prime $\sigma$-ideals in $\mathcal{F}\left\{\mathbb{Y} \backslash\left\{y_{i}\right\}\right\}$. So the theorem is also valid for $\left\{\mathcal{I}, y_{i}\right\}=\left\{\mathcal{I}_{i}, y_{i}\right\}$. The theorem is proved.

\subsection{Characteristic set for normal binomial $\sigma$-ideal}

The theory of characteristic set given in Section 4.2 can be extended to the normal $\sigma$-binomial case.

Let $\rho$ be a partial character over $\mathbb{Z}[x]^{n}, L_{\rho}=\left(\mathbf{f}_{1}, \ldots, \mathbf{f}_{s}\right)$ where $\mathbb{f}=\left\{\mathbf{f}_{1}, \ldots, \mathbf{f}_{s}\right\}$ is a reduced Gröbner basis, and

$$
\mathcal{A}^{+}(\rho): \mathbb{Y}_{1}^{\mathbf{f}_{1}^{+}}-\rho\left(\mathbf{f}_{1}\right) \mathbb{Y}^{\mathbf{f}_{1}^{-}}, \ldots, \mathbb{Y}^{\mathbf{f}_{s}^{+}}-\rho\left(\mathbf{f}_{s}\right) \mathbb{Y}^{\mathbf{f}_{s}^{-}} .
$$

We have the following canonical representation for normal binomial $\sigma$-ideals.

Theorem 5.29 Use the notations in (31). Then $\mathcal{I}^{+}(\rho)=\operatorname{sat}\left(\mathcal{A}^{+}(\rho)\right)$. Furthermore, $\mathcal{A}^{+}(\rho)$ is a regular and coherent $\sigma$-chain and hence is a characteristic set of $\mathcal{I}^{+}(\rho)$.

Proof: Let $\mathcal{I}_{1}=\left[\mathcal{A}^{+}(\rho)\right]$ : m. We claim $\mathcal{I}_{1}=\operatorname{sat}\left(\mathcal{A}^{+}(\rho)\right)$. It is clear that $\operatorname{sat}\left(\mathcal{A}^{+}(\rho)\right) \subset$ $\left[\mathcal{A}^{+}(\rho)\right]: \mathrm{m}=\mathcal{I}_{1}$. For the other direction, let $p \in \mathcal{I}_{1}$ and $p_{1}=\operatorname{prem}\left(p, \mathcal{A}^{+}(\rho)\right)$ which is reduced w.r.t. $\mathcal{A}^{+}(\rho)$. By (2), $p_{1} \in \mathcal{I}_{1}$. As a consequence, $p_{1} \in[\mathcal{A}(\rho)]$ as Laurent $\sigma$ polynomials in $\mathcal{F}\left\{\mathbb{Y}^{ \pm}\right\}$. By Lemma 4.16, $\mathcal{A}(\rho)$ is a characteristic set of $[\mathcal{A}(\rho)]$. Since $p_{1}$ is reduced w.r.t. $\mathcal{A}^{+}(\rho)$, it is also reduced w.r.t. $\mathcal{A}(\rho)$. Then $p_{1}=0$ and hence the claim is proved.

We now prove $\mathcal{I}^{+}(\rho)=\operatorname{sat}\left(\mathcal{A}^{+}(\rho)\right)$. By the above claim, Lemma 4.16, and Lemma 5.16, $\operatorname{sat}\left(\mathcal{A}^{+}(\rho)\right)=\left[\mathcal{A}^{+}(\rho)\right]: \mathrm{m}=\left[\mathcal{A}^{+}(\rho)\right] \mathcal{F}\left\{\mathbb{Y}^{ \pm}\right\} \cap \mathcal{F}\{\mathbb{Y}\}=[\mathcal{A}(\rho)] \cap \mathcal{F}\{\mathbb{Y}\}=\mathcal{I}(\rho) \cap \mathcal{F}\{\mathbb{Y}\}=$ $\mathcal{I}^{+}(\rho)$.

It remains to prove that $\mathcal{A}^{+}(\rho)$ is a characteristic set of $\mathcal{I}_{1}=\left[\mathcal{A}^{+}(\rho)\right]: \mathrm{m}$. By definition, it suffices to show that if $p \in \mathcal{I}_{1}$ is reduced w.r.t. $\mathcal{A}^{+}(\rho)$ then $p=0$. Let $A_{i}=\mathbb{Y}^{\mathbf{f}_{i}}-\rho\left(\mathbf{f}_{i}\right)$ and $A_{i}^{+}=\mathbb{Y}_{i}^{\mathbf{f}_{i}^{+}}-\rho\left(\mathbf{f}_{i}\right) \mathbb{Y}_{i_{i}^{-}}$. Since $p \in \mathcal{I}_{1}$, there exist a $\sigma$-monomial $M$ and $f_{i, j} \in \mathcal{F}\{\mathbb{Y}\}$ such that $M p=\sum_{i, j} f_{i, j}\left(A_{i}^{+}\right)^{x^{j}}$. Then in $\mathcal{F}\left\{\mathbb{Y}^{ \pm}\right\}$, we have $p=\sum_{i, j} g_{i, j} A_{i}^{x^{j}} \in[\mathcal{A}(\rho)]$, where $g_{i, j} \in \mathcal{F}\left\{\mathbb{Y}^{ \pm}\right\}$. Since $p$ is reduced w.r.t. $\mathcal{A}^{+}(\rho)$, it is also reduced w.r.t. $\mathcal{A}(\rho)$. By Lemma 4.16. $\mathcal{A}(\rho)$ is a characteristic set of $[\mathcal{A}(\rho)]$ and hence $p=0$. The claim is proved.

Since $\mathcal{I}_{1}=\operatorname{sat}\left(\mathcal{A}^{+}(\rho)\right), \mathcal{A}^{+}(\rho)$ is also a characteristic set of $\operatorname{sat}\left(\mathcal{A}^{+}(\rho)\right)$. By Theorem 2.2 , $\mathcal{A}^{+}(\rho)$ is regular and coherent.

Example 5.30 Let $L=\left([1-x, x-1]^{\tau}\right)$ be a $\mathbb{Z}[x]$-module and $\rho$ the trivial partial character on $L$, that is, $\rho(\mathbf{f})=1$ for $\mathbf{f} \in L$. By Theorem 5.29, $\mathcal{I}^{+}(\rho)=\operatorname{sat}\left[y_{1} y_{2}^{x}-y_{1}^{x} y_{2}\right] \subseteq \mathbb{Q}\left\{y_{1}, y_{2}\right\}$. By Theorem 5.16, $\mathcal{I}^{+}(\rho)$ is a reflexive prime $\sigma$-ideal. We can show that $\mathcal{I}^{+}(\rho)=\left[y_{1}^{x^{i}} y_{2}^{x^{j}}-\right.$ $\left.y_{1}^{x^{j}} y_{2}^{x^{i}} \mid 0 \leq i<j \leq m\right]$, which is an infinitely generated $\sigma$-ideal. 
As a consequence of Theorem 5.19, Theorem 5.29, and Lemma 4.16, we have

Corollary 5.31 Let $\mathcal{A}(\rho)$ and $\mathcal{A}^{+}(\rho)$ be defined in (17) and (31), respectively. Then $\left([\mathcal{A}(\rho)] \mathcal{F}\left\{\mathbb{Y}^{ \pm}\right\}\right) \cap \mathcal{F}\{\mathbb{Y}\}=\operatorname{sat}\left(\mathcal{A}^{+}(\rho)\right)$.

In order to prove the converse of Theorem 5.29, we need the following lemmas. Let $\mathbf{f}_{i} \in \mathbb{Z}[x]^{n}$ and $c_{i} \in \mathcal{F}^{*}, i=1, \ldots, s$. Consider the following $\sigma$-chains

$$
\begin{aligned}
\mathcal{A} & : \quad \mathbb{Y}^{\mathbf{f}_{1}}-c_{1}, \ldots, \mathbb{Y}^{\mathbf{f}_{s}}-c_{s} \\
\mathcal{A}^{+} & : \quad \mathbb{Y}^{\mathbf{f}_{1}^{+}}-c_{1} \mathbb{Y}^{\mathbf{f}_{1}^{-}}, \ldots, \mathbb{Y}^{\mathbb{Y}_{s}^{+}}-c_{s} \mathbb{Y}^{\mathbf{f}_{s}^{-}}
\end{aligned}
$$

in $\mathcal{F}\left\{\mathbb{Y}^{ \pm}\right\}$and $\mathcal{F}\{\mathbb{Y}\}$, respectively. Since $\mathbf{f}_{i}$ are assumed to be normal, $\mathcal{A}^{+}$is a $\sigma$-chain if and only if $\mathcal{A}$ is a Laurent $\sigma$-chain.

Lemma 5.32 Use the notations in (32). Let $p=a \mathbb{Y}^{\mathbf{a}}+b \mathbb{Y}^{\mathbf{b}}=a N\left(\mathbb{Y}^{\mathbf{f}}-c\right) \in \mathcal{F}\{\mathbb{Y}\}$, where $\mathbf{a}, \mathbf{b} \in \mathbb{N}[x]^{n}, \mathbf{f} \in \mathbb{Z}[x]^{n}, N$ is a $\sigma$-monomial, $c \in \mathcal{F}^{*}$. If $\mathcal{A}^{+}$is coherent and regular, then $\operatorname{prem}\left(p, \mathcal{A}^{+}\right)=0$ implies $\operatorname{prem}\left(\mathbb{Y}^{\mathbf{f}}-c, \mathcal{A}\right)=0$.

Proof: Since $\operatorname{prem}\left(p, \mathcal{A}^{+}\right)=0$, there exists a $\sigma$-monomial $M_{1}$ such that $M_{1} p \in\left[\mathcal{A}^{+}\right]$. Let $p_{1}=\mathbb{Y}^{\mathbf{f}}-c$. Since $r_{1}=\operatorname{prem}\left(p_{1}, \mathcal{A}\right)=\mathbb{Y}^{\mathbf{g}}-c_{\mathbf{g}}$, by Lemma 4.5, there exists a $c_{1} \in \mathcal{F}^{*}$ such that $r_{1}-c_{1} p_{1} \in[\mathcal{A}]$. Then, there exists a $\sigma$-monomial $M_{2}$ such that $M_{2} N r_{1}, M_{2} N p_{1} \in \mathcal{F}\{\mathbb{Y}\}$ and $M_{2} N\left(r_{1}-c_{1} p_{1}\right) \in\left[\mathcal{A}^{+}\right]$and hence $M_{2} M_{1} N\left(r_{1}-c_{1} p_{1}\right)=M_{2} M_{1} N r_{1}-\frac{c_{1}}{a} M_{2} M_{1} p \in\left[\mathcal{A}^{+}\right]$. Let $M=M_{1} M_{2} N$. From $M_{1} p \in\left[\mathcal{A}^{+}\right]$, we have $M r_{1} \in\left[\mathcal{A}^{+}\right] \subset \operatorname{sat}\left(A^{+}\right)$.

Suppose $A_{i}=\mathbb{Y}^{\mathbf{f}_{i}^{+}}-c \mathbb{Y}^{\mathbf{f}_{i}^{-}}=I_{i}^{+} y_{c_{i}}^{d_{i} x_{i}^{o}}-c I_{i}^{-}$, where $y_{c_{i}}$ is the leading variable of $A_{i}$. A variable like $y_{c_{i}}^{x^{o_{i}+k}}$ for $k \in \mathbb{N}$ is called a main variable of $\mathcal{A}^{+}$. A variable $y_{i}^{x^{j}}$ is called a parameter of $\mathcal{A}^{+}$is it is not a main variable. If $M$ contains a main variable of $\mathcal{A}^{+}$as a factor. Then let $z=y_{c_{i}}^{x^{o_{i}+k}}$ be the largest one appearing in $M$ under the variable ordering induced by the lexicographical of the index $\left(c_{i}, o_{i}+k\right)$. Let $s=\operatorname{deg}(M, z)$ and $M_{1}=M /\left(z^{s}\right)$. We may assume that $d_{i}$ is a factor of $s$. Otherwise, let $s_{1}=\left\lfloor\frac{s}{d_{i}}\right\rfloor, s_{0}=s-s_{1} d_{i}$, and $M=M z^{d_{i}-s_{0}}=$ $M_{1} z^{d_{i}\left(s_{1}+1\right)}$. We still have $M r_{1} \in \operatorname{sat}\left(A^{+}\right)$. We may use $A_{i}=0$ to eliminate $z$ from $M$ as follows: $M_{1} z^{s-d_{i}}\left(c I_{i}^{-}\right)^{x^{k}} r_{1}=M_{1} z^{s-d_{i}}\left(I_{i}^{+} y_{c_{i}}^{d_{i} x_{i}^{o}}-A_{i}\right)^{x^{k}} r_{1}=M\left(I_{i}^{+}\right)^{x^{k}} r_{1}-M_{1} z^{s-d_{i}}\left(A_{i}\right)^{x^{k}} r_{1} \in$ $\operatorname{sat}\left(A^{+}\right)$. Note that $\operatorname{deg}\left(M_{1} z^{s-d_{i}}\left(c I_{i}^{-}\right)^{x^{k}}, z\right)=s-d_{i}$. Repeat the above procedure, we may find a $\sigma$-monomial $N$ such that $N r_{1} \in \operatorname{sat}\left(A^{+}\right), N$ does not contain $z$ as a factor, and any variable $y_{i}^{x^{j}}$ in $M$ is smaller than $z$ in the given variable ordering. Repeat the procedure, we may finally obtain a $\sigma$-monomial $L$ such that $L$ does not contain main variables of $\mathcal{A}^{+}$as factors and $L r_{1} \in \operatorname{sat}\left(A^{+}\right)$. Since $L$ contains only parameters of $\mathcal{A}^{+}$and $r_{1}$ is reduced w.r.t. $\mathcal{A}^{+}, L r_{1}$ is also reduced w.r.t. $\mathcal{A}^{+}$. Since $\mathcal{A}^{+}$is regular and coherent, by Lemma 3.6, it is the characteristic set of $\operatorname{sat}\left(A^{+}\right)$. Therefore, $L r_{1}=0$, and $r_{1}=0$.

The following example shows that if $\operatorname{prem}\left(p, \mathcal{A}^{+}\right) \neq 0$ then the relation between $\operatorname{prem}(p$, $\left.\mathcal{A}^{+}\right)$and $\operatorname{prem}\left(\mathbb{Y}^{\mathbf{f}}-c, \mathcal{A}\right)$ may be complicated, where $p=a \mathbb{Y}^{\mathbf{a}}+b \mathbb{Y}^{\mathbf{b}}=a N\left(\mathbb{Y}^{\mathbf{f}}-c\right)$.

Example 5.33 Let $p=y_{2}\left(y_{2}-1\right), A_{1}=y_{1}^{-1} y_{2}^{2}-1$, and $A_{1}^{+}=y_{2}^{2}-y_{1}$. Then $\operatorname{prem}\left(p, A_{1}^{+}\right)=$ $y_{1}-y_{2}$ in $\mathcal{F}\left\{y_{2}\right\}$. But in $\mathcal{F}\left\{y_{2}^{ \pm}\right\}, p$ is represented as $\widetilde{p}=y_{2}-1$ and $\operatorname{prem}\left(\widetilde{p}, A_{1}\right)=y_{2}-1$. 
Lemma 5.34 Use the notations in (32). $\mathcal{A}$ is a regular and coherent $\sigma$-chain in $\mathcal{F}\left\{\mathbb{Y}^{ \pm}\right\}$if and only if $\mathcal{A}^{+}$is a regular and coherent $\sigma$-chain in $\mathcal{F}\{\mathbb{Y}\}$.

Proof: If $\mathcal{A}$ is regular and coherent, by Corollary 4.20, there exists a partial character $\rho$ over $\mathbb{Z}[x]^{n}$ such that $L_{\rho}=\left(\mathbf{f}_{1}, \ldots, \mathbf{f}_{s}\right), \rho\left(\mathbf{f}_{i}\right)=c_{i}$, and $\mathcal{I}(\rho)=[\mathcal{A}]$. By Theorem 5.29, $\mathcal{A}^{+}=\mathcal{A}^{+}(\rho)$ is regular and coherent.

Assume that $\mathcal{A}^{+}$is regular and coherent. We first show that $[\mathcal{A}] \neq[1]$ in $\mathcal{F}\left\{\mathbb{Y}^{ \pm}\right\}$. It suffices to show that $\operatorname{sat}\left(\mathcal{A}^{+}\right)$does not contain a $\sigma$-monomial. Suppose the contrary, there is a $\sigma$-monomial $M \in \operatorname{sat}\left(\mathcal{A}^{+}\right)$. Since $\mathcal{A}^{+}$is a regular and coherent chain, we have $\operatorname{prem}\left(M, \mathcal{A}^{+}\right)=0$. Now consider the procedure of prem, it can be shown that the pseudoremainder of a nonzero $\sigma$-monomial w.r.t. a binomial $\sigma$-chain is still a nonzero $\sigma$-monomial, a contradiction.

Note that $\mathcal{A}$ is always regular since $\sigma$-monomials are invertible in $\mathcal{F}\left\{\mathbb{Y}^{ \pm}\right\}$. Then, it suffices to prove that $\mathcal{A}$ is coherent.

Let $A_{i}=\mathbb{Y}^{\mathbf{f}_{i}}-c_{i}$ and $A_{i}^{+}=\mathbb{Y}_{i}^{\mathbf{f}^{+}}-c_{i} \mathbb{Y}^{\mathbf{f}_{i}^{-}}$. Assume $A_{i}^{+}$and $A_{j}^{+}(i<j)$ have the same leading variable $y_{l}$, and $A_{i}^{+}=I_{i}^{+} y_{l}^{d_{i} x^{o_{i}}}-c_{i} I_{i}^{-}, A_{j}^{+}=I_{j}^{+} y_{l}^{d_{j} x^{o_{j}}}-c_{j} I_{j}^{-}$, where $I_{i}^{-}=\mathbb{Y}^{\mathbf{f}_{i}^{-}}$. From Definition [3.8, we have $o_{i}<o_{j}$ and $d_{i} \mid d_{j}$. Let $d_{i}=t d_{j}$ where $t \in \mathbb{N}$. From (33),

$$
\Delta\left(A_{i}^{+}, A_{j}^{+}\right)=\operatorname{prem}\left(\left(A_{i}^{+}\right)^{x^{o_{j}-o_{i}}}, A_{j}^{+}\right)=c_{j}^{t}\left(I_{j}^{-}\right)^{t}\left(I_{i}\right)^{x^{o_{j}-o_{i}}}-\left(I_{j}^{+}\right)^{t}\left(c_{i} I_{i}^{+}\right)^{x^{o_{j}-o_{i}}} .
$$

Comparing to (15), if $\Delta\left(A_{i}, A_{j}\right)=\mathbb{Y}^{\mathbf{h}}-c_{f}$, then $\Delta\left(A_{i}^{+}, A_{j}^{+}\right)=c_{j}^{t} M\left(\mathbb{Y}^{\mathbf{h}^{+}}-c_{f} \mathbb{Y}^{\mathbf{h}^{-}}\right)$, where $M$ is a $\sigma$-monomial. Since $\mathcal{A}^{+}$is coherent, $\operatorname{prem}\left(\Delta\left(A_{i}^{+}, A_{j}^{+}\right), \mathcal{A}^{+}\right)=0$. By Lemma 5.32 , $\operatorname{prem}\left(\Delta\left(A_{i}, A_{j}\right), \mathcal{A}\right)=0$ which implies that $\mathcal{A}$ is coherent.

We now prove the converse of Theorem 5.29.

Theorem 5.35 Use the notations in (32). If $\mathcal{A}^{+}$is a regular and coherent $\sigma$-chain, then there is a partial character $\rho$ over $\mathbb{Z}[x]^{n}$ such that $L_{\rho}=\left(\mathbf{f}_{1}, \ldots, \mathbf{f}_{s}\right), \rho\left(\mathbf{f}_{i}\right)=c_{i}, \mathcal{I}(\rho)=[\mathcal{A}]$, and $\mathcal{I}^{+}(\rho)=\operatorname{sat}\left(\mathcal{A}^{+}\right)$

Proof: By Lemma 5.34, $\mathcal{A}$ is regular and coherent. By Theorem 4.14, $\mathrm{f}$ is a reduced Gröbner basis for a $\mathbb{Z}[x]$-lattice and $[\mathcal{A}] \subset \mathcal{F}\left\{\mathbb{Y}^{ \pm}\right\}$is proper. By Corollary 4.20 , there exists a partial character $\rho$ such that $L_{\rho}=\left(\mathbf{f}_{1}, \ldots, \mathbf{f}_{s}\right), \rho\left(\mathbf{f}_{i}\right)=c_{i}$, and $\mathcal{I}(\rho)=[\mathcal{A}]$. By Theorem 5.29 , $\mathcal{I}^{+}(\rho)=\operatorname{sat}\left(\mathcal{A}^{+}(\rho)\right)=\operatorname{sat}\left(\mathcal{A}^{+}\right)$.

As a consequence of Theorem 5.35 and Lemma 5.16, we have

Corollary 5.36 Normal binomial $\sigma$-ideals are in a one to one correspondence with $\operatorname{sat}\left(\mathcal{A}^{+}\right)$, where $\mathcal{A}^{+}$is a regular and coherent chain given in (32).

As a consequence of Corollary 5.31 and Theorem 5.35, we have

Corollary 5.37 $\mathcal{F}\left\{\mathbb{Y}^{ \pm}\right\}[\mathcal{A}] \cap \mathcal{F}\{\mathbb{Y}\}=\operatorname{sat}\left(\mathcal{A}^{+}\right)$.

As a consequence of Theorem 4.23, Corollary 5.22, and Theorem 5.35, 
Corollary $5.38[\mathcal{A}]$ is a reflexive (prime) $\sigma$-ideal in $\mathcal{F}\left\{\mathbb{Y}^{ \pm}\right\}$if and only if $\operatorname{sat}\left(\mathcal{A}^{+}\right)$is a reflexive (prime) $\sigma$-ideal in $\mathcal{F}\{\mathbb{Y}\}$.

\subsection{Perfect closure of binomial $\sigma$-ideal and binomial $\sigma$-variety}

In this section, we will show that the perfect closure of a binomial $\sigma$-ideal is also binomial. We will also give a geometric description of the zero set of a binomial $\sigma$-ideal. For the perfect closure of a binomial $\sigma$-ideal, we have

Theorem 5.39 Let $\mathcal{F}$ be an algebraically closed and inversive $\sigma$-field. Then the perfect closure of a binomial $\sigma$-ideal $\mathcal{I}$ is binomial.

We first remark that it is not known wether the well-mixed closure of a binomial $\sigma$-ideal is still binomial. Before proving Theorem 5.39, we first prove several lemmas. In the rest of this section, we assume that $\mathcal{I} \subseteq S=\mathcal{F}\{\mathbb{Y}\}$ and $\mathfrak{m}$ the set of $\sigma$-monomials in $S$.

Lemma 5.40 If $\mathcal{I}$ is a binomial $\sigma$-ideal, then $\{\mathcal{I}\}: \mathfrak{m}$ is either $[1]$ or a binomial $\sigma$-ideal.

Proof: It is easy to check $\{\mathcal{I}\} \mathcal{F}\left\{\mathbb{Y}^{ \pm}\right\}=\left\{\mathcal{I} \mathcal{F}\left\{\mathbb{Y}^{ \pm}\right\}\right\}$. By (27), $\{\mathcal{I}\}: \mathfrak{m}=\{\mathcal{I}\} \mathcal{F}\left\{\mathbb{Y}^{ \pm}\right\} \cap \mathcal{F}\{\mathbb{Y}\}=$ $\left\{\mathcal{I} \mathcal{F}\left\{\mathbb{Y}^{ \pm}\right\}\right\} \cap \mathcal{F}\{\mathbb{Y}\}$. Now the lemma follows from Theorem 4.52,

Lemma 5.41 If $\mathcal{I}$ is a $\sigma$-ideal in $\mathcal{F}\{\mathbb{Y}\}$, then

$$
\{\mathcal{I}\}=\{\mathcal{I}\}: \operatorname{m} \cap\left\{\mathcal{I}+y_{1}\right\} \cap \cdots \cap\left\{\mathcal{I}+y_{n}\right\}
$$

Proof: The right hand side of (33) clearly contains $\{\mathcal{I}\}$. It suffices to show that every reflexive prime $P$ containing $\mathcal{I}$ contains one of the $\sigma$-ideals on the right-hand side of (33). If $\{\mathcal{I}\}: \mathrm{m} \subseteq P$, we are done. Otherwise, there exists an element $f \in(\{\mathcal{I}\}: \mathrm{m}) \backslash P$ which implies that there exists a $\sigma$-monomial $M$ such that $M f \in\{\mathcal{I}\} \subseteq P$. This implies $y_{i} \in P$ for some $i$. Thus, $P$ contains $\left\{\mathcal{I}+y_{i}\right\}$ as required.

Lemma 5.42 Let $\mathcal{I}$ be a binomial $\sigma$-ideal in $S=\mathcal{F}\{\mathbb{Y}\}$ and $S^{\prime}=\mathcal{F}\left\{y_{1}, \ldots, y_{n-1}\right\}$. If $\mathcal{I}^{\prime}=\mathcal{I} \cap S^{\prime}$, then $\left[\mathcal{I}+y_{n}\right]$ is the sum of $\left[\mathcal{I}^{\prime} S+y_{n}\right]$ and a monomial $\sigma$-ideal in $S^{\prime}$.

Proof: Every $\sigma$-binomial involving $y_{n}^{x^{k}}$ is either contained in $\left[y_{n}\right]$ or is congruent modulo $\left[y_{n}\right]$ to a $\sigma$-monomial in $S^{\prime}$. Thus, all generators of $\mathcal{I}$ which are not in $\mathcal{I}^{\prime}$ may be replaced by $\sigma$-monomials in $S^{\prime}$ when forming a generating set for $\left[\mathcal{I}+y_{n}\right]$.

Lemma 5.43 Let $\mathcal{I}$ be a perfect binomial $\sigma$-ideal in $S=\mathcal{F}\{\mathbb{Y}\}$. If $\mathcal{M}$ is a $\sigma$-monomial $\sigma$-ideal, then $\{\mathcal{I}+\mathcal{M}\}=\left[\mathcal{I}+\mathcal{M}_{1}\right]$ for some monomial $\sigma$-ideal $\mathcal{M}_{1}$.

Proof: If $1 \in \mathcal{M}$, then the lemma is obviously valid. Otherwise, $[\mathcal{I}+\mathcal{M}]: \mathbb{m}=[1]$. Lemma 5.41 yields $\{\mathcal{I}+\mathcal{M}\}=\bigcap_{i=1}^{i=n}\left\{\mathcal{I}+\mathcal{M}+y_{i}\right\}$. By Corollary [5.9, we need only to show that $\left\{\mathcal{I}+\mathcal{M}+y_{i}\right\}$ is the sum of $\mathcal{I}$ and a monomial $\sigma$-ideal. For simplicity, let $i=n$ and write $S^{\prime}=\mathcal{F}\left\{y_{1}, y_{2}, \ldots, y_{n-1}\right\}$. Since $\mathcal{I}$ is perfect, the $\sigma$-ideal $\mathcal{I}^{\prime}=\mathcal{I} \cap S^{\prime}$ is perfect as well. By 
Lemma 5.42, $\left[\mathcal{I}+\mathcal{M}+y_{n}\right]=\left[\mathcal{I}^{\prime} S+\mathcal{M}^{\prime} S+y_{n}\right]$ where $\mathcal{M}^{\prime}$ is a monomial $\sigma$-ideal in $S^{\prime}$. By induction on $n$, the perfect closure of $\mathcal{I}^{\prime}+\mathcal{M}^{\prime}$ in $S^{\prime}$ has the form $\mathcal{I}^{\prime}+\mathcal{M}_{1}^{\prime}$, where $\mathcal{M}_{1}^{\prime}$ is a monomial $\sigma$-ideal of $S^{\prime}$. Putting this together, we have

$$
\begin{aligned}
\left\{\mathcal{I}+\mathcal{M}+y_{n}\right\} & =\left\{\mathcal{I}^{\prime} S+\mathcal{M}^{\prime} S+y_{n}\right\} \\
& =\left[\mathcal{I}^{\prime} S+\mathcal{M}_{1}^{\prime} S+y_{n}\right] \\
& \subseteq\left[\mathcal{I}+\mathcal{M}_{1}^{\prime} S+y_{n}\right] \\
& \subseteq\left\{\mathcal{I}+\mathcal{M}+y_{n}\right\}
\end{aligned}
$$

So $\left\{\mathcal{I}+\mathcal{M}+y_{n}\right\}=\left[\mathcal{I}+\mathcal{M}_{1}^{\prime} S+y_{n}\right]$ is $\mathcal{I}$ plus a monomial $\sigma$-ideal, as required.

Proof of Theorem 5.39: We will prove the theorem by induction on $n$. By Lemma 5.40, $\mathcal{I}_{1}=\{\mathcal{I}\}: \mathrm{m}$ is binomial. For $n=1$, by Lemma 5.41. $\{\mathcal{I}\}=\mathcal{I}_{1} \cap\left\{\mathcal{I}+y_{1}\right\}$. If $\left\{\mathcal{I}+y_{1}\right\}=1$ then $\{\mathcal{I}\}=\mathcal{I}_{1}$ is binomial. Otherwise $\{\mathcal{I}+y\}=[y]$ and hence $\mathcal{I} \subset[y]$. Since $\mathcal{I} \subset \mathcal{I}_{1}$, $\{\mathcal{I}\}=\mathcal{I}_{1} \cap[y]=\left[\mathcal{I}+\mathcal{I}_{1}\right] \cap[\mathcal{I}+y]$ is binomial by Lemma 5.8. Suppose the lemma is valid for $n-1$ variables and let $\mathcal{I}$ be a binomial $\sigma$-ideal in $S=\mathcal{F}\{\mathbb{Y}\}$. Let $\mathcal{I}_{j}:=\mathcal{I} \cap S_{j}$, where $S_{j}=\mathcal{F}\left\{y_{1}, \ldots, y_{j-1}, y_{j+1}, \ldots, y_{n}\right\}$. By the induction hypothesis, we may assume that the perfect closure of each $\mathcal{I}_{j}$ is binomial. Adding these binomial $\sigma$-ideals to $\mathcal{I}$, we may assume that each $\mathcal{I}_{j}$ is perfect begin with. By Lemma 5.40, $\mathcal{I}_{1}=\{\mathcal{I}\}: \mathrm{m}$ is binomial. Then there exists a binomial $\sigma$-ideal $\mathcal{I}^{\prime}$, say $\mathcal{I}^{\prime}=\mathcal{I}_{1}$, such that $\mathcal{I}_{1}=\left[\mathcal{I}+\mathcal{I}^{\prime}\right]$. By Lemma 5.42 , $\left[\mathcal{I}+y_{j}\right]=\left[\mathcal{I}_{j} S+\mathcal{J}_{j} S+y_{j}\right]$, where $\mathcal{J}_{j}$ is a monomial $\sigma$-ideal in $S_{j}$. Since $\mathcal{I}_{j}$ is perfect, the $\sigma$ ideal $\mathcal{I}_{j} S$ is perfect, so we can apply Lemma 5.43 with $\mathcal{M}=\left[\mathcal{J}_{j} S+y_{i}\right]$ to see that there exists a monomial $\sigma$-ideal $\mathcal{M}_{j}$ in $S$ such that $\left\{\mathcal{I}+y_{j}\right\}=\left\{\mathcal{I}_{j} S+\mathcal{J}_{j} S+y_{j}\right\}=\left[\mathcal{I}_{j} S+\mathcal{M}_{j}\right]=\left[\mathcal{I}+\mathcal{M}_{j}\right]$. By Lemma 5.41 and Corollary 5.8, $\{\mathcal{I}\}=\left[\mathcal{I}+\mathcal{I}^{\prime}\right] \cap \cap_{j=1}^{n}\left[\mathcal{I}+\mathcal{M}_{j}\right]$ is binomial.

Example 5.44 Let $p=y_{2}^{2}-y_{1}^{2}$. Following the proof of Theorem [5.39, $\{p\}=(\{p\}: \mathfrak{m}) \cap$ $\left[y_{1}, y_{2}\right]$. By Example 4.48 and Corollary 5.37, $\mathcal{I}_{1}=\{p\}: \mathfrak{m}=\operatorname{sat}\left[y_{2}^{2}-y_{1}^{2}, y_{1} y_{2}^{x}-y_{1}^{x} y_{2}\right]=$ $\left[y_{1} y_{2}^{x^{i}}-y_{1}^{x^{i}} y_{2}, y_{2}^{1+x^{j}}-y_{1}^{1+x^{j}} \mid i, j \in \mathbb{N}\right]$. Thus, $\{p\}=\mathcal{I}_{1} \cap\left[y_{1}, y_{2}\right]=\mathcal{I}_{1}$.

A $\sigma$-ideal $\mathcal{I}$ is called normal if for any $M \in \mathrm{m}$ and $p \in \mathcal{F}\{\mathbb{Y}\}, M p \in \mathcal{I}$ implies $p \in \mathcal{I}$. The following example shows that the perfect closure of a normal $\sigma$-ideal could be not normal.

Example 5.45 Let $\mathcal{I}=\left[y_{2}^{2} y_{4}^{2}-y_{1}^{2} y_{3}^{2}, y_{1}^{x}-y_{1}, y_{2}^{x}-y_{2}\right]$. By Example 5.44, we have $\{\mathcal{I}\}=$ $\left[p_{i},\left(y_{2} y_{4}\right)^{1+x^{j}}-\left(y_{1} y_{3}\right)^{1+x^{j}}, y_{1}^{x}-y_{1}, y_{2}^{x}-y_{2} \mid i, j \in \mathbb{N}\right]$, where $p_{i}=y_{1} y_{3}\left(y_{2} y_{4}\right)^{x^{i}}-y_{2} y_{4}\left(y_{1} y_{3}\right)^{x^{i}}$. Note that $p_{i}=y_{1} y_{2}\left(y_{3} y_{4}^{x^{i}}-y_{4} y_{3}^{x^{i}}\right)$ modulo $\left[y_{1}^{x}-y_{1}, y_{2}^{x}-y_{2}\right]$ and $y_{3} y_{4}^{x^{i}}-y_{4} y_{3}^{x^{i}}$ is not in $\{\mathcal{I}\}$.

In the rest of this section, we give a geometric description of the zero set of a binomial $\sigma$-ideal, which is a generalization of Theorem 4.1 in [7] to the difference case. The basic idea of the proof also follows [7], except we need to consider the distinction between the perfect $\sigma$-ideals and radical ideals.

We decompose the affine $n$-space $(\mathbb{A})^{n}$ into the union of $2^{n} \sigma$-coordinate flats:

$$
\left(\mathbb{A}^{*}\right)^{\Omega}:=\left\{\left(a_{1}, a_{2}, \ldots, a_{n}\right) \mid a_{i} \neq 0, i \in \Omega ; a_{i}=0, i \notin \Omega\right\}
$$

where $\Omega$ runs over all subsets of $\{1,2, \ldots, n\}$. The Cohn closure of $\left(\mathbb{A}^{*}\right)^{\Omega}$ in $(\mathbb{A})^{n}$ is defined by the $\sigma$-ideal

$$
M(\Omega):=\left[y_{i} \mid i \notin \Omega\right] \subset \mathcal{F}\{\mathbb{Y}\} .
$$


The $\sigma$-coordinate ring of $\left(\mathbb{A}^{*}\right)^{\Omega}$ is the Laurent polynomial $\sigma$-ring $\mathcal{F}\left\{\Omega^{ \pm}\right\}:=\mathcal{F}\left\{y_{i}, y_{i}^{-1}, i \in \Omega\right\}$. We can define a coordinate projection $\left(\mathbb{A}^{*}\right)^{\Omega^{\prime}} \longrightarrow\left(\mathbb{A}^{*}\right)^{\Omega}$ whenever $\Omega \subseteq \Omega^{\prime} \subseteq\{1,2, \ldots, n\}$ by setting all those coordinates not in $\Omega$ to zero.

If $X$ is any $\sigma$-variety of $(\mathbb{A})^{n}$ and $\mathcal{I}=\mathbb{I}(X) \subseteq \mathcal{F}\{\mathbb{Y}\}$, then the Cohn closure of the intersection of $X$ with $\left(\mathbb{A}^{*}\right)^{\Omega}$ corresponds to the $\sigma$-ideal

$$
\mathcal{I}_{\Omega}:=[\mathcal{I}+M(\Omega)]: \mathfrak{m}_{\Omega} \subset \mathcal{F}\{\mathbb{Y}\}
$$

where $\mathfrak{m}_{\Omega}=\left\{\prod_{i \in \Omega} y_{i}^{m_{i}(x)} \mid m_{i}(x) \in \mathbb{N}[x]\right\}$. Since $\mathcal{I}$ is perfect, by the difference Nullstellsatz [3, p.87]

$$
\mathcal{I}=\bigcap_{\Omega}\left\{\mathcal{I}_{\Omega}\right\}
$$

If $\mathcal{I}$ is binomial, then by Corollary 5.10 the $\sigma$-ideal $\mathcal{I}_{\Omega}$ is also binomial.

Lemma 5.46 Let $R:=\mathcal{F}\left\{z_{1}, z_{1}^{-1}, \ldots, z_{t}, z_{t}^{-1}\right\} \subset R^{\prime}:=\mathcal{F}\left\{z_{1}, z_{1}^{-1}, \ldots, z_{t}, z_{t}^{-1}, y_{1}, \ldots, y_{s}\right\}$ be a Laurent polynomial $\sigma$-ring and a polynomial $\sigma$-ring over it. If $B \subset R^{\prime}$ is a binomial $\sigma$-ideal and $M \subset R^{\prime}$ is a monomial $\sigma$-ideal such that $[B+M]$ is a proper $\sigma$-ideal in $R^{\prime}$, then

$$
[B+M] \cap R=B \cap R .
$$

Proof: This is a $\sigma$-version of [7, Lemma 4.2], which can be proved similarly.

We can make a classification of all binomial $\sigma$-varieties $X$ by intersecting $X$ with $\left(\mathbb{A}^{*}\right)^{\Omega}$, since by Theorem 5.39, the perfect closure of a binomial $\sigma$-ideal is still binomial.

Theorem 5.47 Let $\mathcal{F}$ be any algebraically closed and inversive $\sigma$-field. A $\sigma$-variety $X \subset \mathbb{A}^{n}$ is generated by $\sigma$-binomials if and only if the following three conditions hold.

(1) For each $\left(\mathbb{A}^{*}\right)^{\Omega}$, the $\sigma$-variety $X \cap\left(\mathbb{A}^{*}\right)^{\Omega}$ is generated by $\sigma$-binomials.

(2) The family of sets $U=\left\{\Omega \subseteq\{1,2, \ldots, n\} \mid X \cap\left(\mathbb{A}^{*}\right)^{\Omega} \neq \emptyset\right\}$ is closed under taking intersections.

(3) If $\Omega_{1}, \Omega_{2} \in U$ and $\Omega_{1} \subset \Omega_{2}$, then the coordinate projection $\left(\mathbb{A}^{*}\right)^{\Omega_{2}} \longrightarrow\left(\mathbb{A}^{*}\right)^{\Omega_{1}}$ maps $X \cap\left(\mathbb{A}^{*}\right)^{\Omega_{2}}$ onto a subset of $X \cap\left(\mathbb{A}^{*}\right)^{\Omega_{1}}$.

The above theorem can be reduced to the following algebraic version.

Theorem 5.48 Let $\mathcal{F}$ be any algebraically closed and inversive $\sigma$-field. A perfect $\sigma$-ideal $\mathcal{I} \subset \mathcal{F}\{\mathbb{Y}\}$ is binomial if and only if the following three conditions hold.

(1) For each $\Omega \subseteq\{1, \ldots, n\}, \mathcal{I}_{\Omega}$ is binomial.

(2) $U=\left\{\Omega \subseteq\{1,2, \ldots, n\} \mid\left\{\mathcal{I}_{\Omega}\right\} \neq[1]\right\}$ is closed under taking intersections.

(3) If $\Omega_{1}, \Omega_{2} \in U$ and $\Omega_{1} \subset \Omega_{2}$, then $\mathcal{I}_{\Omega_{1}} \cap \mathcal{F}\left\{\Omega_{1}\right\} \subset \mathcal{I}_{\Omega_{2}}$, where $\mathcal{F}\left\{\Omega_{1}\right\}=\mathcal{F}\left\{y_{i} \mid y_{i} \in \Omega_{1}\right\}$. 
Proof: Suppose $\mathcal{I}$ is a perfect $\sigma$-ideal in $\mathcal{F}\{\mathbb{Y}\}$. Since $\mathcal{I}$ is binomial, by Lemma $5.10 \mathcal{I}_{\Omega}$ is also binomial and (1) is proved. To prove (2) by contradiction, assume that for $\Omega_{1}, \Omega_{2} \in U$, $\left\{\mathcal{I}_{\Omega_{1}}\right\} \neq[1],\left\{\mathcal{I}_{\Omega_{2}}\right\} \neq[1],\left\{\mathcal{I}_{\Omega_{1} \cap \Omega_{2}}\right\}=[1]$. We consider two cases. If $\mathcal{I}_{\Omega_{1} \cap \Omega_{2}}=[1]$, then for some $m(x) \in \mathbb{N}[x]$ we have $\left(\prod_{i \in \Omega_{1} \cap \Omega_{2}} y_{i}\right)^{m(x)} \in\left[\mathcal{I}+M\left(\Omega_{1}\right)+M\left(\Omega_{2}\right)\right]$. By Corollary [5.9, $\left(\prod_{i \in \Omega_{1} \cap \Omega_{2}} y_{i}\right)^{m(x)}$ is either in $\left[\mathcal{I}+M\left(\Omega_{1}\right)\right]$ or $\left[\mathcal{I}+M\left(\Omega_{2}\right)\right]$, so $\mathcal{I}_{\Omega_{1}}$ or $\mathcal{I}_{\Omega_{2}}$ is [1]. For the second case, we have $\mathcal{I}_{\Omega_{1} \cap \Omega_{2}} \neq[1]$ and $\left\{\mathcal{I}_{\Omega_{1} \cap \Omega_{2}}\right\}=[1]$. Then there exist a finite number of proper $\sigma$-binomials $B_{1}, \ldots, B_{s}$ and $\sigma$-monomials $m_{1}, \ldots, m_{s}$ in $\mathcal{F}\left\{\Omega_{1} \cap \Omega_{2}\right\}$ such that $m_{i} B_{i} \in \mathcal{I}$ and $\left\{B_{1}, \ldots, B_{s}, y_{i}, i \notin \Omega_{1} \cap \Omega_{2}\right\}=[1]$. We thus have $\left\{B_{1}, \ldots, B_{s}\right\}=[1]$. Since $m_{i} B_{i} \in$ $\mathcal{I} \cap \mathcal{F}\left\{\Omega_{1} \cap \Omega_{2}\right\}$, we have $B_{i} \in \mathcal{I}_{\Omega_{1}}$ and $B_{i} \in \mathcal{I}_{\Omega_{2}}$ and thus $\left\{\mathcal{I}_{\Omega_{1}}\right\}=\left\{\mathcal{I}_{\Omega_{2}}\right\}=$ [1]. To prove (3), given $\Omega_{1}, \Omega_{2} \in U$ and $\Omega_{1} \subset \Omega_{2}$, we have $\mathcal{I}_{\Omega_{2}}=\left[\mathcal{I}_{\Omega_{2}}: \mathfrak{m}_{\Omega_{1}}\right]$. Set $R^{\prime}=\mathcal{F}\left\{\Omega_{1}^{ \pm}\right\}\left\{\left\{y_{i}\right\}_{i \notin \Omega_{1}}\right\}$, then

$$
\left[\mathcal{I}+M\left(\Omega_{1}\right)\right] R^{\prime} \cap \mathcal{F}\left\{\Omega_{1}^{ \pm}\right\} \subseteq \mathcal{I}_{\Omega_{2}} R^{\prime} .
$$

Since $\Omega_{1} \in U$, the $\sigma$-ideal $\left[\mathcal{I}+M\left(\Omega_{1}\right)\right] R^{\prime}$ is proper. By Lemma 5.46, we have $\left[\mathcal{I}+M\left(\Omega_{1}\right)\right] R^{\prime} \cap$ $\mathcal{F}\left\{\Omega_{1}^{ \pm}\right\}=\mathcal{I} R^{\prime} \cap \mathcal{F}\left\{\Omega_{1}^{ \pm}\right\} \subset \mathcal{I}_{\Omega_{2}} R^{\prime} \cap \mathcal{F}\left\{\Omega_{1}^{ \pm}\right\}$. So $\mathcal{I}_{\Omega_{1}} \cap \mathcal{F}\left\{\Omega_{1}\right\} \subset \mathcal{I}_{\Omega_{2}}$.

To prove the other driection, let $\mathcal{I}$ be a perfect $\sigma$-ideal satisfying the three conditions. By the difference Nullstellensatz, $\mathcal{I}=\cap_{\Omega \in U}\left\{\mathcal{I}_{\Omega}\right\}$. By condition (2), $U$ is a partially ordered set under the inclusion for subsets of $\{1, \ldots, n\}$. For each $\Omega \in U$, we set $\mathcal{J}(\Omega)=\left[\mathcal{I}_{\Omega} \cap\right.$ $\mathcal{F}\{\Omega\}] \mathcal{F}\{\mathbb{Y}\}$ with the properties that if $\Omega_{1} \subset \Omega_{2},\left\{\mathcal{J}\left(\Omega_{1}\right)\right\} \subset\left\{\mathcal{J}\left(\Omega_{2}\right)\right\}$. Note that $\left[M_{\Omega_{1} \cap \Omega_{2}}\right] \subset$ $\left[M_{\Omega_{1}}+M_{\Omega_{2}}\right]$. Then we have

$$
\mathcal{I}=\cap_{\Omega \in U}\left\{\mathcal{I}_{\Omega}\right\}=\cap_{\Omega \in U}\{\mathcal{J}(\Omega)+M(\Omega)\} .
$$

Now we will prove that

$$
\cap_{\Omega \in U}\{\mathcal{J}(\Omega)+M(\Omega)\}=\left\{\cap_{\Omega \in U} M(\Omega)+\sum_{\Omega \in U}\left\{\mathcal{J}(\Omega) \cap\left(\cap_{\Omega_{\eta} \nsupseteq \Omega} M\left(\Omega_{\eta}\right)\right)\right\}\right\} .
$$

If $\Omega_{2} \supseteq \Omega_{1}$, we have $\left\{\mathcal{J}\left(\Omega_{2}\right)+M\left(\Omega_{2}\right)\right\} \supseteq\left\{\mathcal{J}\left(\Omega_{2}\right)\right\} \supseteq\left\{\mathcal{J}\left(\Omega_{1}\right)\right\} \supseteq\left\{\mathcal{J}\left(\Omega_{1}\right) \cap \cap_{\Omega_{\eta} \nsupseteq \Omega_{1}} M\left(\Omega_{\eta}\right)\right\}$. If $\Omega_{2} \nsupseteq \Omega_{1}$, we have $\left\{\mathcal{J}\left(\Omega_{2}\right)+M\left(\Omega_{2}\right)\right\} \supseteq M\left\{\Omega_{2}\right\} \supseteq\left\{\mathcal{J}\left(\Omega_{1}\right) \cap \cap_{\Omega_{\eta} \nsupseteq \Omega_{1}} M\left(\Omega_{\eta}\right)\right\}$. So the left hand side contains the right hand side of (34). For the other direction, consider a reflexive prime $\sigma$-ideal $P \supseteq\left[\cap_{\Omega \in U} M(\Omega)+\sum_{\Omega \in U}\left\{\mathcal{J}(\Omega) \cap \cap_{\Omega_{\eta} \not \Omega} M\left(\Omega_{\eta}\right)\right\}\right]$ and set $V=$ $\{\Omega \in U \mid M(\Omega) \subset P\}$. Then $V$ is a finite partially order set and nonempty since $P \supseteq$ $\cap_{\Omega \in U} M(\Omega)$ and $\left\{M_{\Omega_{1} \cap \Omega_{2}}\right\} \subset\left\{M_{\Omega_{1}}+M_{\Omega_{2}}\right\}$. Let $\Omega_{0}$ be the smallest element of $V$ such that $P \supseteq M_{\Omega_{0}}$. At the same time, $P \supset \mathcal{J}\left(\Omega_{0}\right) \cap \cap_{\Omega_{\eta} \nsupseteq \Omega_{0}} M\left(\Omega_{\eta}\right)$, then $P \supseteq \mathcal{J}\left(\Omega_{0}\right)$. Therefore, $P \supseteq \mathcal{J}\left(\Omega_{0}\right)+M\left(\Omega_{0}\right)$ and $P$ contains the left hand side (34) and (34) is proved. Since $\cap_{\Omega \in U} M(\Omega)+\sum_{\Omega \in U}\left\{\mathcal{J}(\Omega) \cap \cap_{\Omega_{\eta} \nsupseteq \Omega} M\left(\Omega_{\eta}\right)\right\}$ is binomial, the theorem follows from (34).

\section{Toric $\sigma$-ideal and toric $\sigma$-variety}

In this section, we will introduce the concept of toric $\sigma$-variety and prove some of its basic properties. 


\subsection{Toric $\sigma$-variety}

Let $\mathcal{F}$ be an inversive $\sigma$-field. Following [33, we denote the category of $\sigma$-field extensions of $\mathcal{F}$ by $\mathscr{E}_{\mathcal{F}}$, the category of $\mathcal{E}^{n}$ by $\mathscr{E}_{\mathcal{F}}^{n}$ where $\mathcal{E} \in \mathscr{E}_{\mathcal{F}}$. Let $\left(\mathbb{A}^{*}\right)^{n}$ be the functor from $\mathscr{E}_{\mathcal{F}}$ to $\mathscr{E}_{\mathcal{F}}$ satisfying $\left(\mathbb{A}^{*}\right)^{n}(\mathcal{E})=\left(\mathcal{E}^{*}\right)^{n}$ where $\mathcal{E} \in \mathscr{E}_{\mathcal{F}}$ and $\mathcal{E}^{*}=\mathcal{E} \backslash\{0\}$. Let $\mathbb{A}^{n}$ be the functor from $\mathscr{E}_{\mathcal{F}}$ to $\mathscr{E}_{\mathcal{F}}^{n}$ satisfying $(\mathbb{A})^{n}(\mathcal{E})=(\mathcal{E})^{n}$ where $\mathcal{E} \in \mathscr{E}_{\mathcal{F}}$. A $\sigma$-variety over $\mathcal{F}$ is a functor $\mathbb{V}$ from $\mathscr{E}_{\mathcal{F}}$ to the category of sets with the form $\mathbb{V}(P)$ for $P \subset \mathcal{F}\{\mathbb{Y}\}$ satisfying $\mathbb{V}_{\mathcal{E}}(P)=\left\{\eta \in \mathcal{E}^{n} \mid \forall p \in P, p(\eta)=0\right\}$.

In the rest of this section, let

$$
\mathbb{Q}=\left\{\boldsymbol{\alpha}_{1}, \ldots, \boldsymbol{\alpha}_{n}\right\}, \text { where } \boldsymbol{\alpha}_{i} \in \mathbb{Z}[x]^{m}, i=1, \ldots, n,
$$

and $\mathbb{T}=\left(t_{1}, \ldots, t_{m}\right)$ a set of $\sigma$-indeterminates. We define the following rational $\sigma$-morphism

$$
\phi:\left(\mathbb{A}^{*}\right)^{m} \longrightarrow\left(\mathbb{A}^{*}\right)^{n}, \mathbb{T} \mapsto \mathbb{T}^{\mathbb{Q}}=\left(\mathbb{T}^{\boldsymbol{\alpha}_{1}}, \ldots, \mathbb{T}^{\boldsymbol{\alpha}_{n}}\right) .
$$

Define the functor $T_{\mathbb{Q}}$ from $\mathscr{E}_{\mathcal{F}}$ to $\mathscr{E}_{\mathcal{F}}$ with $T_{\mathbb{Q}}(\mathcal{E})=\operatorname{Im}\left(\phi_{\mathcal{E}}\right)$ which is called a quasi $\sigma$-torus with defining vector $\mathbb{\alpha}$. For each $\mathcal{E} \in \mathscr{E}_{\mathcal{F}}, T_{\mathbb{Q}}(\mathcal{E})$ has a group structure with component wise multiplication: $\mathbb{T}_{1}^{\mathbb{Q}} \cdot \mathbb{T}_{2}^{\mathbb{Q}}=\left(\mathbb{T}_{1} \cdot \mathbb{T}_{2}\right)^{\mathbb{Q}}$ where $\mathbb{T}_{1}, \mathbb{T}_{2} \in\left(\mathcal{E}^{*}\right)^{m}$ and $\boldsymbol{\alpha} \in \mathbb{Z}[x]^{m}$. If $\mathcal{E}_{1}, \mathcal{E}_{2} \in \mathscr{E}_{\mathcal{F}}$, $\mathcal{E}_{1} \subseteq \mathcal{E}_{2}$, then $T_{\mathbb{Q}}\left(\mathcal{E}_{1}\right)$ is a subgroup of $T_{\mathbb{Q}}\left(\mathcal{E}_{2}\right)$.

We now define the toric $\sigma$-variety.

Definition 6.1 A $\sigma$-variety over the $\sigma$-field $\mathbb{Q}$ is called toric if it is the Cohn closure of a quasi $\sigma$-torus $T_{\mathbb{Q}}$ in $\mathbb{A}^{n}$, where $\mathbb{\alpha}$ is given in (35). More precisely, let

$$
\mathcal{I}_{\mathbb{Q}}=\left\{f \in \mathbb{Q}\{\mathbb{Y}\} \mid f\left(\mathbb{T}^{\boldsymbol{\alpha}_{1}}, \ldots, \mathbb{T}^{\boldsymbol{\alpha}_{n}}\right)=0\right\} .
$$

Then the toric $\sigma$-variety defined by $\mathbb{Q}=\left\{\boldsymbol{\alpha}_{1}, \ldots, \boldsymbol{\alpha}_{n}\right\}$ is $\mathbf{X}_{\mathbb{Q}}=\mathbb{V}\left(\mathcal{I}_{\mathbb{Q}}\right) . A=\left[\boldsymbol{\alpha}_{1}, \ldots, \boldsymbol{\alpha}_{n}\right]_{m \times n}$ is called the defining matrix of $\mathbf{X}_{\mathbb{Q}}$.

We make the following reasonable assumption: $A$ does not contain a zero row, or equivalently, every $t_{i}$ appears effectively in some $\mathbb{T}^{\boldsymbol{\alpha}_{k}}$. Also, no $\boldsymbol{\alpha}_{i}$ is the zero vector.

Lemma 6.2 Let $L_{\mathbb{Q}}$ be the $\mathbb{Z}[x]$-lattice generated by $\propto$ given in (35). Then $\mathbf{X}_{\mathbb{Q}}$ is an irreducible $\sigma$-variety of dimension $\operatorname{rk}\left(L_{\mathbb{Q}}\right)$.

Proof: It is clear that $\mathbb{T}^{\mathbb{Q}}$ in (36) is a generic point of $\mathcal{I}_{\mathbb{Q}}$ in (37). Then $\mathcal{I}_{\mathbb{Q}}$ is a reflexive prime $\sigma$-ideal. By Theorem 3.20 of $[21,22], \mathcal{I}_{\mathbb{Q}}$ is of dimension $\triangle \operatorname{tr} \cdot \operatorname{deg} \mathbb{Q}\left\langle\mathbb{T}^{\mathbb{Q}}\right\rangle / \mathbb{Q}=\operatorname{rk}(A)=\operatorname{rk}\left(L_{\mathbb{Q}}\right)$, where $A=\left[\boldsymbol{\alpha}_{1}, \ldots, \boldsymbol{\alpha}_{n}\right]_{m \times n}$ is the defining matrix of $\mathbf{X}_{\mathbb{Q}}$.

Let $\left[y_{1}-\mathbb{T}^{\boldsymbol{\alpha}_{1}}, \ldots, y_{n}-\mathbb{T}^{\boldsymbol{\alpha}_{n}}\right]$ be the $\sigma$-ideal generated by $y_{i}-M_{i}, i=1, \ldots, n$ in $\mathbb{Q}\left\{\mathbb{Y}, \mathbb{T}^{ \pm}\right\}$. Then it is easy to check

$$
\mathcal{I}_{\mathbb{Q}}=\left[y_{1}-\mathbb{T}^{\boldsymbol{\alpha}_{1}}, \ldots, y_{n}-\mathbb{T}^{\boldsymbol{\alpha}_{n}}\right] \cap \mathbb{Q}\{\mathbb{Y}\} .
$$

Alternatively, let $\left[\mathbb{T}^{\boldsymbol{\alpha}_{1}^{+}} y_{1}-\mathbb{T}^{\boldsymbol{\alpha}_{1}^{-}}, \ldots, \mathbb{T}^{\boldsymbol{\alpha}_{n}^{+}} y_{n}-\mathbb{T}^{\boldsymbol{\alpha}_{n}^{-}}\right]$be a $\sigma$-ideal in $\mathbb{Q}\{\mathbb{Y}, \mathbb{T}\}$. Then

$$
\mathcal{I}_{\mathbb{Q}}=\left[\mathbb{T}^{\boldsymbol{\alpha}_{1}^{+}} y_{1}-\mathbb{T}^{\boldsymbol{\alpha}_{1}^{-}}, \ldots, \mathbb{T}^{\boldsymbol{\alpha}_{n}^{+}} y_{n}-\mathbb{T}^{\boldsymbol{\alpha}_{n}^{-}}\right]: \mathrm{m}_{\mathbb{T}} \cap \mathbb{Q}\{\mathbb{Y}\}
$$

where $\mathrm{m}_{\mathbb{T}}$ is the multiplicative set generated by $t_{i}^{x^{j}}$ for $i=1, \ldots, m, j \in \mathbb{N}$. 
Remark 6.3 With formulas (38) and (39), a characteristic set for $\mathcal{I}_{\mathbb{Q}}$ can be computed with the characteristic set method in [10]. More efficient methods to compute a characteristic set for $\mathcal{I}_{\mathbb{Q}}$ will be given in Section 6.2.

Example 6.4 Let $M_{2}=\left[\begin{array}{llll}2 & x-1 & 0 & 0 \\ 0 & 0 & 2 & x-1\end{array}\right]$ be the matrix from Example 3.11 and $\mathbb{\alpha}$ the set of columns of $M_{2}$. Let $\mathcal{I}_{1}=\left[y_{1}-t_{1}^{2}, y_{2}-t_{1}^{x-1}, y_{3}-t_{2}^{2}, y_{4}-t_{2}^{x-1}\right]$. By (38), $\mathcal{I}_{\mathbb{Q}}=$ $\mathcal{I}_{1} \cap \mathbb{Q}\left\{y_{1}, y_{2}, y_{3}, y_{4}\right\}$. With the characteristic set method [10], under the variable order $y_{2}<$ $y_{4}<y_{1}<y_{3}<t_{1}<t_{2}$, a characteristic set of $\mathcal{I}_{1}$ is $y_{1} y_{2}^{2}-y_{1}^{x}, y_{3} y_{4}^{2}-y_{3}^{x}, y_{1}-t_{1}^{2}, t_{1} y_{2}-t_{1}^{x}, y_{3}-$ $t_{2}^{2}, t_{2} y_{4}-t_{2}^{x}$. Then

$$
\mathcal{I}_{\mathbb{Q}}=\operatorname{sat}\left(y_{1} y_{2}^{2}-y_{1}^{x}, y_{3} y_{4}^{2}-y_{3}^{x}\right)=\left[y_{1} y_{2}^{2}-y_{1}^{x}, y_{3} y_{4}^{2}-y_{3}^{x}\right] .
$$

The following example shows that some $y_{i}$ might not appear effectively in $\mathcal{I}_{\mathbb{Q}}$.

Example 6.5 Let $\mathbb{Q}=\left\{[1,1]^{\tau},[x, x]^{\tau},[0,1]^{\tau}\right\}$. By (38), $\mathcal{I}_{\mathbb{Q}}=\left[y_{1}-t_{1} t_{2}, y_{2}-t_{1}^{x} t_{2}^{x}, y_{3}-t_{2}\right]$ $\cap \mathbb{Q}\left\{y_{1}, y_{2}, y_{3}\right\}=\left[y_{1}^{x}-y_{2}\right]$ and $y_{3}$ does not appear in $\mathcal{I}_{\mathbb{Q}}$.

The following lemma shows that quasi $\sigma$-tori of two sets of generators of $L_{\mathbb{Q}}$ are isomorphic.

Lemma 6.6 Let $\alpha=\left\{\boldsymbol{\alpha}_{1}, \ldots, \boldsymbol{\alpha}_{n}\right\}$ and $\boldsymbol{\beta}=\left\{\boldsymbol{\beta}_{1}, \ldots, \boldsymbol{\beta}_{s}\right\}$ be two sets of generators for $L$. Then $T_{\mathbb{Q}}$ and $T_{\mathbb{\beta}}$ are isomorphic as groups.

Proof: Let $A_{m \times n}$ and $B_{m \times s}$ be the matrix representations for the two sets of generators for $L$. Then there exist matrices $M=\left(m_{i j}\right) \in \mathbb{Z}[x]^{s \times n}$ and $N=\left(n_{i j}\right) \in \mathbb{Z}[x]^{n \times s}$ such that $A=B M, B=A N$. Hence $A=A N M, B=B M N$. Define two maps $\theta_{1}: \mathbb{A}^{n} \Rightarrow$ $\mathbb{A}^{s}$ and $\theta_{2}: \mathbb{A}^{s} \Rightarrow \mathbb{A}^{n}$ by $\theta_{1}\left(y_{1}, \ldots, y_{n}\right)=\left(\prod_{i=1}^{n} y_{i}^{n_{i 1}}, \ldots, \prod_{i=1}^{n} y_{i}^{n_{i s}}\right)$ and $\theta_{2}\left(x_{1}, \ldots, x_{s}\right)=$ $\left(\prod_{i=1}^{s} x_{i}^{m_{i 1}}, \ldots, \prod_{i=1}^{s} x_{i}^{m_{i n}}\right)$. Then it is clear that $\theta_{1}\left(T_{\mathbb{Q}}\right) \subset T_{\beta}$ and $\theta_{2}\left(T_{\beta}\right) \subset T_{\mathbb{Q}}$. From $A=A N M$ and $B=B M N$, it can be checked that $\theta_{1} \circ \theta_{2}=i d$ on quasi $\sigma$-torus $T_{\beta}$ and $\theta_{2} \circ \theta_{1}=i d$ on quasi $\sigma$-torus $T_{\mathbb{Q}}$. It is easy to check $\theta_{1}$ and $\theta_{2}$ are group homomorphisms. Therefore, the quasi tori $T_{\mathbb{Q}}$ and $T_{\mathbb{\beta}}$ are isomorphic as groups.

In the rest of this section, we will give a description for the coordinate ring of a toric $\sigma$-variety.

Definition 6.7 $M \subseteq \mathbb{Z}[x]^{m}$ is called an affine $\mathbb{N}[x]$-module if there exists $a \boldsymbol{\beta}=\left\{\boldsymbol{\beta}_{1}, \ldots, \boldsymbol{\beta}_{s}\right\}$ $\subset \mathbb{Z}[x]^{m}$ such that $M=\mathbb{N}[x](\mathbb{\beta})=\left\{\sum_{i=1}^{s} a_{i} \boldsymbol{\beta}_{i} \mid a_{i} \in \mathbb{N}[x]\right\}$.

Given an affine $\mathbb{N}[x]$-module $M \subseteq \mathbb{Z}[x]^{m}$ and a set of $\sigma$-indeterminates $\mathbb{T}=\left\{t_{1}, \ldots, t_{m}\right\}$, define the corresponding affine $\sigma$-algebra

$$
\mathcal{F}\{M\}=\left\{\sum_{\boldsymbol{\beta} \in M} a_{\boldsymbol{\beta}^{\mathbb{T}^{\boldsymbol{\beta}}}} a_{\boldsymbol{\beta}} \in \mathcal{F}, a_{\boldsymbol{\beta}} \neq 0 \text { for finitely many } \boldsymbol{\beta}\right\} .
$$

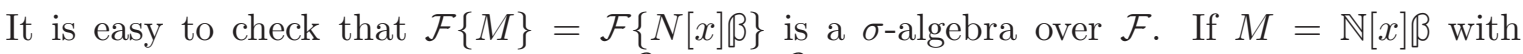
$\boldsymbol{\beta}=\left\{\boldsymbol{\beta}_{1}, \ldots, \boldsymbol{\beta}_{s}\right\}$, then $\mathcal{F}\{M\}=\mathcal{F}\left\{\mathbb{T} \boldsymbol{\beta}_{1}, \ldots, \mathbb{T} \boldsymbol{\beta}_{s}\right\}$. 
Example 6.8 As an affine $\mathbb{N}[x]$-module, $\mathbb{N}[x]^{m}$ gives the $\sigma$-polynomial ring in $\mathbb{T}: \mathcal{F}\left\{\mathbb{N}[x]^{m}\right\}$ $=\mathcal{F}\{\mathbb{T}\}$. Let $\boldsymbol{\epsilon}_{1}, \ldots, \boldsymbol{\epsilon}_{m}$ be the standard basis of dimension $m$. Then $\mathbb{Z}[x]^{m}$ is generated by $A=\left\{ \pm \boldsymbol{\epsilon}_{1}, \ldots, \pm \boldsymbol{\epsilon}_{m}\right\}$ as an affine $\mathbb{N}[x]$-module, and the corresponding affine $\sigma$-algebra is the Laurent $\sigma$-polynomial ring $\mathcal{F}\left\{\mathbb{Z}[x]^{m}\right\}=\mathcal{F}\left\{\mathbb{T}^{ \pm}\right\}$.

Given an affine $\mathbb{N}[x]$-module $M \subseteq \mathbb{Z}[x]^{n}$, we define $\operatorname{Spec}^{\sigma}(\mathcal{F}\{M\})$ to be the set of reflexive prime $\sigma$-ideals of $\mathcal{F}\{M\}$ [33].

Theorem 6.9 $X$ is a toric $\sigma$-variety if and only if there exists an affine $\mathbb{N}[x]$-module $M$ such that $X \cong \operatorname{Spec}^{\sigma}(\mathbb{Q}\{M\})$. Equivalently, the coordinate ring of $X$ is $\mathbb{Q}\{M\}$.

Proof: Let $X=\mathbf{X}_{\mathbb{Q}}$ and $\mathbb{I}(X)=\mathcal{I}_{\mathbb{Q}}$, where $\mathbb{\alpha}$ is defined in (35) and $\mathcal{I}_{\mathbb{Q}}$ is defined in (37). Let $M=\mathbb{N}[X] \propto$ be the affine $\mathbb{N}[x]$-module generated by $\mathbb{\alpha}$. Define the following morphism of $\sigma$-rings

$$
\theta: \mathbb{Q}\{\mathbb{Y}\} \longrightarrow \mathbb{Q}\{M\}, \text { where } \theta\left(y_{i}\right)=\mathbb{T}^{\boldsymbol{\alpha}_{i}}, i=1, \ldots, n .
$$

The map $\theta$ is surjective by the definition of $\mathbb{Q}\{M\}$. If $f \in \operatorname{ker}(\theta)$, then $f\left(\mathbb{T}^{\boldsymbol{\alpha}_{1}}, \ldots, \mathbb{T}^{\boldsymbol{\alpha}_{i}}\right)=0$, which is equivalent to $f \in \mathcal{I}_{\mathbb{Q}}$. Then, $\operatorname{ker}(\theta)=\mathcal{I}_{\mathbb{Q}}$ and $\mathbb{Q}\{\mathbb{Y}\} / \mathcal{I}_{\mathbb{Q}} \cong \mathbb{Q}\{M\}$. Therefore $X \cong \operatorname{Spec}^{\sigma}\left(\mathbb{Q}\{\mathbb{Y}\} / \mathcal{I}_{\mathbb{Q}}\right)=\operatorname{Spec}^{\sigma}(\mathbb{Q}\{M\})$.

If $X \cong \operatorname{Spec}^{\sigma}(\mathbb{Q}\{M\})$, where $M \subseteq \mathbb{Z}^{m}[x]$ is an affine $\mathbb{N}[x]$-module, and $M=\mathbb{N}[x]\left(\boldsymbol{\alpha}_{1}, \ldots\right.$, $\left.\boldsymbol{\alpha}_{n}\right)$ for $\boldsymbol{\alpha}_{i} \in M$. Let $\mathbf{X}_{\mathbb{Q}}$ be the toric $\sigma$-variety defined by $\mathbb{Q}=\left\{\boldsymbol{\alpha}_{1}, \ldots, \boldsymbol{\alpha}_{n}\right\}$. Then as we just proved, the coordinate ring of $X$ is isomorphic to $\mathbb{Q}\{M\}$. Then $X \cong \mathbf{X}_{\mathbb{Q}}$.

\subsection{Toric $\sigma$-ideal}

In this section, we will show that $\sigma$-toric varieties are defined exactly by toric $\sigma$-ideals which are in a one to one correspondence with toric $\mathbb{Z}[x]$-lattices.

Definition 6.10 A $\mathbb{Z}[x]$-lattice $L \subseteq \mathbb{Z}[x]^{n}$ is called toric or $\mathbb{Z}[x]$-saturated if for any $p \in \mathbb{Z}[x]$ and $\mathbf{f} \in \mathbb{Z}[x]^{n}$, pf $\in L$ implies $\mathbf{f} \in L$. Let $L$ be a toric $\mathbb{Z}[x]$-lattice in $\mathbb{Z}[x]^{n}$ and $\rho_{L}$ the trivial partial character defined on $L$. Then the $\sigma$-ideal

$$
I^{+}\left(\rho_{L}\right)=\left[\mathbb{Y}^{\mathbf{f}^{+}}-\mathbb{Y}^{\mathbf{f}^{-}} \mid \mathbf{f} \in L\right]=\left[\mathbb{Y}^{\mathbf{a}}-\mathbb{Y}^{\mathbf{b}} \mid \mathbf{a}-\mathbf{b} \in L, \mathbf{a}, \mathbf{b} \in \mathbb{N}[x]^{n}\right]
$$

is called a toric $\sigma$-ideal.

We list several properties for toric $\sigma$-ideals.

- By Theorem 4.23, a toric $\sigma$-ideal is a reflexive prime $\sigma$-ideal of dimension $n-\operatorname{rk}(L)$.

- By Corollary 5.17, toric $\sigma$-ideals in $\mathcal{F}\{\mathbb{Y}\}$ are in a one to one correspondence with toric lattices in $\mathbb{Z}[x]^{n}$.

In Section [7.3, an algorithm will be given to check whether a $\mathbb{Z}[x]$-lattice is toric. In this section, we will prove the following result which can be deduced from Lemmas 6.12 and 6.16. 
Theorem 6.11 A $\sigma$-variety $V$ over $\mathbb{Q}$ is toric if and only if $\mathbb{I}(V)$ is a toric $\sigma$-ideal.

The following lemma shows that the defining ideal of a toric $\sigma$-variety is a toric $\sigma$-ideal.

Lemma 6.12 Let $\mathbf{X}_{\mathbb{Q}}$ be a toric $\sigma$-variety and $A$ the defining matrix of $\mathbf{X}_{\mathbb{Q}}$. Then $\mathbb{I}\left(\mathbf{X}_{\mathbb{Q}}\right)$ is the toric $\sigma$-ideal whose support lattice is $\operatorname{ker}(A)$.

Proof: Let $A=\left[\boldsymbol{\alpha}_{1}, \ldots, \boldsymbol{\alpha}_{n}\right]_{m \times n}$ be the defining matrix of the toric $\sigma$-variety and

$$
K_{A}=\operatorname{ker}(A)=\left\{\mathbf{f} \in \mathbb{Z}[x]^{n} \mid A \mathbf{f}=0\right\} .
$$

As a kernel, $K_{A}$ is clearly a toric $\mathbb{Z}[x]$-lattice. Then it suffices to show that $\mathbb{I}\left(\mathbf{X}_{\alpha}\right)=\mathcal{I}_{\mathbb{Q}}=$ $\mathcal{I}^{+}\left(\rho_{K_{A}}\right)$, where $\rho_{K_{A}}$ is the trivial partial character defined over $K_{A}$ and $\mathcal{I}_{\mathbb{Q}}$ is defined in (37).

For $\mathbf{f} \in K_{A}$, we have $\left(\mathbb{Y}^{\mathbf{f}}-1\right)\left(\mathbb{T}^{\mathbb{Q}}\right)=\left(\mathbb{T}^{\mathbb{Q}}\right)^{\mathbf{f}}-1=\mathbb{T}^{A \mathbf{f}}-1=0$. As a consequence, $\left(\mathbb{Y}^{\mathbf{f}^{+}}-\mathbb{Y}^{\mathbf{f}^{-}}\right)\left(\mathbb{T}^{\mathbb{Q}}\right)=0$ and $\mathbb{Y}^{\mathbf{f}^{+}}-\mathbb{Y}^{\mathbf{f}^{-}} \in \mathcal{I}_{\mathbb{Q}}$. Since $\mathcal{I}^{+}\left(\rho_{K_{A}}\right)$ is generated by $\mathbb{Y}^{\mathbf{f}^{+}}-\mathbb{Y}^{\mathbf{f}^{-}}$for $\mathbf{f} \in K_{A}$, we have $\mathcal{I}^{+}\left(\rho_{K_{A}}\right) \subset \mathcal{I}_{\mathbb{Q} \text {. }}$.

Consider a well order for elements in $\mathbb{N}^{n}[x]$, which leads to a well order for $\left\{\mathbb{Y}^{\mathbf{f}}, \mathbf{f} \in \mathbb{N}[x]^{n}\right\}$ as well as an order for $\mathcal{F}\{\mathbb{Y}\}$ by comparing the largest $\sigma$-monomial in a $\sigma$-polynomial. We will prove $\mathcal{I}_{\mathbb{Q}} \subset \mathcal{I}^{+}\left(\rho_{K_{A}}\right)$. Assume the contrary, and let $f=\Sigma_{i} a_{i} \mathbb{Y}^{\mathbf{f}_{i}} \in \mathcal{I}_{\mathbb{Q}}$ be a minimal element in $\mathcal{I}_{\mathbb{Q}} \backslash \mathcal{I}^{+}\left(\rho_{K_{A}}\right)$ under the above order. Let $a_{0} \mathbb{Y}^{\mathbf{g}}$ be the biggest $\sigma$-monomial in $f$. From $f \in \mathcal{I}_{\mathbb{Q}}$, we have $f\left(\mathbb{T}^{\mathbb{Q}}\right)=0$. Since $\mathbb{Y}^{\mathbf{g}}\left(\mathbb{T}^{\mathbb{Q}}\right)=\mathbb{T}^{A \mathrm{~g}}$ is a $\sigma$-monomial about $\mathbb{T}$, there exists another $\sigma$-monomial $b_{0} \mathbb{Y}^{\mathbf{h}}$ in $f$ such that $\mathbb{Y}^{\mathbf{h}}\left(\mathbb{T}^{\mathbb{Q}}\right)=\mathbb{Y}^{\mathbf{g}}\left(\mathbb{T}^{\mathbb{Q}}\right)$. As a consequence, $\left(\mathbb{Y}^{\mathbf{g}}-\mathbb{Y}^{\mathbf{h}}\right)\left(\mathbb{T}^{\mathbb{Q}}\right)=\mathbb{T}^{A \mathbf{h}}\left(\mathbb{T}^{A(\mathbf{g}-\mathbf{h})}-1\right)=0$, from which we deduce $\mathbf{g}-\mathbf{h} \in K_{A}$ and hence $\mathbb{Y}^{\mathbf{g}}-\mathbb{Y}^{\mathbf{h}} \in \mathcal{I}_{\mathbb{Q}} \cap \mathcal{I}^{+}\left(\rho_{K_{A}}\right)$. Then $f-a_{0}\left(\mathbb{Y}^{\mathbf{g}}-\mathbb{Y}^{\mathbf{h}}\right) \in \mathcal{I}_{\mathbb{Q}} \backslash \mathcal{I}^{+}\left(\rho_{K_{A}}\right)$, which contradicts to the minimal property of $f$, since $f-a_{0}\left(\mathbb{Y}^{\mathbf{g}}-\mathbb{Y}^{\mathbf{h}}\right)<f$.

To prove the converse of Lemma 6.12, we first introduce a new concept for $\mathbb{Z}[x]$-lattices. Let $L \subset \mathbb{Z}[x]^{n}$ be a $\mathbb{Z}[x]$-lattice. Define the orthogonal complement of $L$ to be

$$
L^{C}=\left\{\mathbf{f} \in \mathbb{Z}[x]^{n} \mid \forall \mathbf{g} \in L,\langle\mathbf{f}, \mathbf{g}\rangle=0\right\}
$$

where $\langle\mathbf{f}, \mathbf{g}\rangle=\mathbf{f}^{\tau} \cdot \mathbf{g}$ is the dot product of $\mathbf{f}$ and $\mathbf{g}$. It is easy to show that

Lemma 6.13 Let $A_{n \times r}$ be a matrix representation for $L$. Then $L^{C}=\operatorname{ker}\left(A^{\tau}\right)=\{\mathbf{f} \in$ $\left.\mathbb{Z}[x]^{n} \mid A^{\tau} \mathbf{f}=0\right\}$ and hence $\operatorname{rk}\left(L^{C}\right)=n-\operatorname{rk}(L)$.

Furthermore, we have

Lemma 6.14 If $L$ is a toric $\mathbb{Z}[x]$-lattice, then $L=\left(L^{C}\right)^{C}$.

Proof: It is easy to see $L \subset\left(L^{C}\right)^{C}$. Let $r=\operatorname{rk}(L)$. By Lemma6.13, $\operatorname{rk}\left(\left(L^{C}\right)^{C}\right)=n-(n-r)=$ $r=\operatorname{rk}(L)$. Let $\mathbb{K}=\mathbb{Q}(x)$. In $\mathbb{K}^{n}$, the $\mathbb{Z}[x]$-lattices $L$ and $\left(L^{C}\right)^{C}$ become vector spaces $\widetilde{L}$ and $\widetilde{\left(L^{C}\right)^{C}}$ with $\operatorname{dim}(\widetilde{L})=\operatorname{rk}(L)=\operatorname{dim}\left(\widetilde{\left(L^{C}\right)^{C}}\right)$. Since $\widetilde{L} \subset \widetilde{\left(L^{C}\right)^{C}}$ and $\operatorname{dim}(\widetilde{L})=\operatorname{dim}\left(\widetilde{\left.\left(L^{C}\right)^{C}\right)}\right.$, we have $\widetilde{L}=\widetilde{\left(L^{C}\right)^{C}}$. Let $\mathbf{f} \in\left(L^{C}\right)^{C}$. Then $\mathbf{f} \in \widetilde{\left(L^{C}\right)^{C}}=\widetilde{L}$, which means that there exists a $p \in \mathbb{Z}[x]$ such that $p \mathbf{f} \in L$. Since $L$ is toric, we have $\mathbf{f} \in L$ and the lemma is proved.

From Lemmas 3.16, 6.13, and 6.14, we have 
Corollary 6.15 A toric $\mathbb{Z}[x]$-lattice is a free $\mathbb{Z}[x]$-module.

The following lemma shows that the inverse of Lemma 6.12 is also valid.

Lemma 6.16 If $\mathcal{I}$ is a toric $\sigma$-ideal in $\mathbb{Q}\{\mathbb{Y}\}$, then $\mathbb{V}(\mathcal{I})$ is a toric $\sigma$-variety.

Proof: Since $\mathcal{I}$ is a toric $\sigma$-ideal, $\mathcal{I}$ is reflexive prime and $\mathbb{L}(\mathcal{I})$ is a toric lattice. Let $\left\{\mathbf{f}_{1}, \ldots, \mathbf{f}_{s}\right\}$ be a set of generators of $\mathbb{L}(\mathcal{I}), F=\left[\mathbf{f}_{1}, \ldots, \mathbf{f}_{s}\right]_{n \times s} \in \mathbb{Z}[x]^{n \times s}$ a matric representation for $\mathbb{L}(\mathcal{I})$, and $K_{F}=\left\{X \in \mathbb{Z}[x]^{n} \mid F^{\tau} X=0\right\}=\mathbb{L}(\mathcal{I})^{C}$. Then by Corollary 6.15 and Lemma 6.13, $K_{F}$ is a free $\mathbb{Z}[x]$-module and hence has a basis $\left\{\mathbf{h}_{1}, \ldots, \mathbf{h}_{n-r}\right\}$, where $r=\operatorname{rk}(F)=\operatorname{rk}(\mathbb{L}(\mathcal{I}))$. Let $H=\left[\mathbf{h}_{1}, \ldots, \mathbf{h}_{n-r}\right]_{n \times(n-r)}$ be the matrix with $\mathbf{h}_{i}$ as the $i$-th column and $\mathbb{\alpha}=\left\{\boldsymbol{\alpha}_{1}, \ldots, \boldsymbol{\alpha}_{n}\right\}$ the rows of $H$. Consider the toric $\sigma$-variety $\mathbf{X}_{\mathbb{Q}}$ defined by the following quasi $\sigma$-torus

$$
\phi:\left(\mathbb{A}^{*}\right)^{n-r} \longrightarrow \mathbb{A}^{n}
$$

where $\phi(\mathbb{T})=\left(\mathbb{T}^{\boldsymbol{\alpha}_{1}}, \ldots, \mathbb{T}^{\boldsymbol{\alpha}_{n}}\right)$ for $\mathbb{T}=\left(t_{1}, \ldots, t_{n-r}\right)$. To prove the lemma, it is suffices to show $\mathbf{X}_{\mathbb{Q}}=\mathbb{V}(\mathcal{I})$ or $\mathcal{I}_{\mathbb{Q}}=\mathcal{I}$

By Lemma 6.12, $\mathcal{I}_{\mathbb{Q}}$ is toric. Since both $\mathcal{I}_{\mathbb{Q}}$ and $\mathcal{I}$ are toric, to prove $\mathcal{I}_{\mathbb{Q}}=\mathcal{I}$, we need only to show $\mathbb{L}\left(\mathcal{I}_{\mathbb{Q}}\right)=\mathbb{L}(\mathcal{I})$. By Lemma 6.13, $K_{F}^{C}=\operatorname{ker}\left(H^{\tau}\right)$. Then, by Lemma 6.12, $\mathbb{L}\left(\mathcal{I}_{\mathbb{Q}}\right)=\operatorname{ker}\left(H^{\tau}\right)=K_{F}^{C}=\left(\mathbb{L}(\mathcal{I})^{C}\right)^{C}$. By Lemma 6.14, $\mathbb{L}\left(\mathcal{I}_{\mathbb{Q}}\right)=\mathbb{L}(\mathcal{I})$ and the lemma is proved.

It should be noted that the proofs of Lemma 6.12 and Lemma 6.13 also give algorithms to compute the defining ideal for a toric $\sigma$-variety and the defining matrix for the toric variety defined by a toric $\sigma$-ideal. In other words, a toric $\sigma$-variety is a $\sigma$-variety which has a Laurent $\sigma$-monomial parametrization, and the proofs of Lemma 6.12 and Lemma 6.13 give implicitization and parametrization algorithms for these kind of unirational $\sigma$-varieties [12].

Example 6.17 Continue from Example 6.4 Let $\mathbf{f}_{1}=(1-x, 2,0,0)^{\tau}, \mathbf{f}_{2}=(0,0,1-x, 2)^{\tau}$. Then $K_{M_{2}}=\operatorname{ker}\left(M_{2}\right)=\left(\mathbf{f}_{1}, \mathbf{f}_{2}\right) \subseteq \mathbb{Z}^{4}[x]$. By Lemma 6.12 and Theorem [5.29, with the variable order $y_{2}<y_{4}<y_{1}<y_{3}$, we have $\mathcal{I}_{\mathbb{Q}}=\operatorname{sat}\left(y_{1} y_{2}^{2}-y_{1}^{x}, y_{3} y_{4}^{2}-y_{3}^{x}\right)=\left[y_{1} y_{2}^{2}-y_{1}^{x}, y_{3} y_{4}^{2}-\right.$ $\left.y_{3}^{x}\right]$.

Conversely, let $B=\left[\mathbf{f}_{1}, \mathbf{f}_{2}\right]_{4 \times 2}$ be the support lattice of $\mathbb{I}\left(\mathbf{X}_{\mathbb{Q}}\right)$. Then $M_{2}^{\tau}$ is the defining matrix for $\operatorname{ker}\left(B^{\tau}\right)$. By Lemma 6.16, $M_{2}$ is the defining matrix for the toric $\sigma$-variety $\mathbf{X}_{\mathbb{Q}}$.

For any $\mathbb{Z}[x]$-lattice $L \subseteq \mathbb{Z}[x]^{n}$, the $\mathbb{Z}[x]$-saturation of $L$ is defined as

$$
\operatorname{sat}_{\mathbb{Z}[x]}(L)=\left\{\mathbf{f} \in \mathbb{Z}[x]^{n} \mid \exists g \in \mathbb{Z}[x] \text {, s.t. } g \mathbf{f} \in L\right\} .
$$

Similar to Corollary 4.27, it can be shown that $\operatorname{rk}\left(\operatorname{sat}_{\mathbb{Z}[x]}(L)\right)=\operatorname{rk}(L)$. An algorithm to compute the $\mathbb{Z}[x]$ saturation is given in Section 7.3 ,

Example 6.18 Continue from Example 5.30, Let $L=\left([1-x, x-1]^{\tau}\right)$. Then $\operatorname{sat}_{\mathbb{Z}[x]}(L)=$ $\left([1,-1]^{\tau}\right)$. The $\sigma$-ideal $\mathcal{I}=\mathcal{I}^{+}\left(\rho_{L}\right)=\operatorname{sat}\left(y_{1} y_{2}^{x}-y_{1}^{x} y_{2}\right)=\left[y_{1}^{x^{i}} y_{2}^{x^{j}}-y_{1}^{x^{j}} y_{2}^{x^{i}}\right]$ is reflexive prime but not toric. The minimal toric $\sigma$-ideal containing $\mathcal{I}$ is $\left[y_{2}-y_{1}\right]$ with lattice support $\left([1,-1]^{\tau}\right)$. This is quite different from the algebraic case [7], where all prime binomial ideals generated by binomial of the form $\left(\mathbb{Y}^{\mathbf{f}}-\mathbb{Y}^{\mathbf{g}}\right)$ are toric. 


\section{3 $\sigma$-torus and toric $\sigma$-variety in terms of group action}

In this section, we first define the $\sigma$-torus and prove its properties and then give a abstract description for $\sigma$-toric varieties in terms of group actions.

Let $T_{\mathbb{Q}}$ be a quasi $\sigma$-torus and $\mathbf{X}_{\mathbb{Q}}$ the toric $\sigma$-variety defined by $\propto \subset \mathbb{Z}[x]^{m}$. In the algebraic case, $T_{\mathbb{Q}}$ is a variety, that is, $T_{\mathbb{Q}}=\mathbf{X}_{\mathbb{Q}} \cap\left(\mathbb{C}^{*}\right)^{m}$, where $\mathbb{C}$ is the field of complex numbers and $\mathbb{C}^{*}=\mathbb{C} \backslash\{0\}$. The following example shows that this is not valid in the difference case.

Example 6.19 In Example 6.4, $\mathbf{X}_{\mathbb{Q}}=\mathbb{V}\left(\left\{y_{1} y_{2}^{2}-y_{1}^{x}, y_{3} y_{4}^{2}-y_{3}^{x}\right\}\right)$. Let $P=(-1,1,-1,-1)$. Then $P \in \mathbf{X}_{\mathbb{Q}}$. On the other hand, assume $P \in T_{\mathbb{Q}}$ which means $\left(\left(t_{1}\right)^{2},\left(t_{1}\right)^{x-1},\left(t_{2}\right)^{2},\left(t_{2}\right)^{x-1}\right)$ $=(-1,1,-1,-1)$ or the $\sigma$-equations $t_{1}^{2}+1=0, t_{1}^{x}-t_{1}=0, t_{2}^{2}+1=0, t_{2}^{x}+t_{2}=0$ have a solution. From Example 4.24, this is impossible. That is, $T_{\mathbb{Q}} \varsubsetneqq \mathbf{X}_{\mathbb{Q}} \cap\left(\mathbb{C}^{*}\right)^{m}$.

In order to define the $\sigma$-torus, we need to introduce the concept of Cohn $*$-closure. $\left(\mathbb{A}^{*}\right)^{n}$ is isomorphic to the $\sigma$-variety defined by $\mathcal{I}_{0}=\left[y_{1} z_{1}-1, \ldots, y_{n} z_{n}-1\right] \subset \mathcal{F}\{\mathbb{Y}, \mathbb{Z}\}$ in $(\mathbb{A})^{2 n}$. Furthermore, $\sigma$-varieties in $\left(\mathbb{A}^{*}\right)^{n}$ are in a one to one correspondence with affine $\sigma$-varieties contained in $\mathbb{V}\left(\mathcal{I}_{0}\right)$ via the map $\theta:\left(\mathbb{A}^{*}\right)^{n} \Rightarrow(\mathbb{A})^{2 n}$ defined by $\theta\left(a_{1}, \ldots, a_{n}\right)=$ $\left(a_{1}, \ldots, a_{n}, a_{1}^{-1}, \ldots, a_{n}^{-1}\right)$. Let $V \subset\left(\mathbb{A}^{*}\right)^{n}$ and $V_{1}$ the Cohn closure of $\theta(V)$ in $(\mathbb{A})^{2 n}$. Then the $\theta^{-1}\left(V_{1}\right)$ is called the Cohn $*$-closure of $V$.

Example 6.19 gives the motivation for the following definition.

Definition 6.20 A $\sigma$-torus is an $\sigma$-variety which is isomorphic to the Cohn $*$-closure of a quasi $\sigma$-torus in $\left(\mathbb{A}^{*}\right)^{n}$.

Theorem 6.21 Let $T_{\mathbb{Q}}$ be the quasi $\sigma$-torus defined in (36) by $\mathbb{\alpha}, T_{\mathbb{Q}}^{*}$ the Cohn $*$-closure of $T_{\mathbb{Q}}$ in $\left(\mathbb{A}^{*}\right)^{n}$, and $\mathcal{I}_{\mathbb{Q}}$ defined in (37). Then $T_{\mathbb{Q}}^{*}$ is isomorphic to $\operatorname{Spec}^{\sigma}\left(\mathcal{F}\{\mathbb{Y}, \mathbb{Z}\} / \widetilde{\mathcal{I}}_{\mathbb{Q}}\right)$ where $\mathbb{Z}=\left\{z_{1}, \ldots, z_{n}\right\}$ is a set of $\sigma$-indeterminates and $\widetilde{\mathcal{I}}_{\mathbb{Q}}=\left[\mathcal{I}_{\mathbb{Q}}, y_{1} z_{1}-1, \ldots, y_{n} z_{n}-1\right]$ in $\mathcal{F}\{\mathbb{Y}, \mathbb{Z}\}$.

Proof: Use the notations just introduced. Let $\widetilde{T}_{\mathbb{Q}}=\theta\left(T_{\mathbb{Q}}\right) \subset \mathbb{A}^{2 n}$ and $\widetilde{T}_{\mathbb{Q}}^{*}$ the Cohn closure of $\widetilde{T}_{\mathbb{Q}}$ in $\mathbb{A}^{2 n}$. Then $T_{\mathbb{Q}}^{*}=\theta^{-1}\left(\widetilde{T}_{\mathbb{Q}}^{*}\right)$ is the Cohn $*$-closure of $T_{\mathbb{Q}}$ in $\left(\mathbb{A}^{*}\right)^{n}$. Since $\theta$ is clearly an isomorphism between $\widetilde{T}_{\mathbb{Q}}^{*}$ and $T_{\mathbb{Q}}^{*}$, it suffices to show that $\mathbb{I}\left(\widetilde{T}_{\mathbb{Q}}^{*}\right)=\widetilde{\mathcal{I}}_{\mathbb{Q}}$.

We have $\mathbb{I}\left(\widetilde{T}_{\mathbb{Q}}^{*}\right)=\left\{p \in \mathcal{F}\{\mathbb{Y}, \mathbb{Z}\} \mid p\left(\mathbb{T}^{\boldsymbol{\alpha}_{1}}, \ldots, \mathbb{T}^{\boldsymbol{\alpha}_{n}}, \mathbb{T}^{-\boldsymbol{\alpha}_{1}}, \ldots, \mathbb{T}^{-\boldsymbol{\alpha}_{n}}\right)=0\right\}$. It is clear that $\widetilde{\mathcal{I}}_{\mathbb{Q}} \subset \mathbb{I}\left(\widetilde{T}_{\mathbb{Q}}^{*}\right)$. If $f \in \mathbb{I}\left(T_{\mathbb{Q}}^{*}\right)$, eliminate $z_{1}, \ldots, z_{n}$ from $f$ by taking the $\sigma$-remainder $f_{1}=$ $\operatorname{prem}\left(f,\left\{y_{1} z_{1}-1, \ldots, y_{n} z_{n}-1\right\}\right)=\prod_{i=1}^{n} y_{i}^{t_{i}} f-\sum_{i=1}^{n} \sum_{j=0}^{l_{i}} g_{i j}\left(y_{i} z_{i}-1\right)^{x^{s_{i j}}} \in \mathcal{F}\{\mathbb{Y}\} \cap \mathbb{I}\left(T_{\mathbb{Q}}^{*}\right)$, where $t_{i} \in \mathbb{N}[x], s_{i j} \in \mathbb{N}, g_{i j} \in \mathcal{F}\{\mathbb{Y}, \mathbb{Z}\}$. Substituting $y_{i}$ by $\mathbb{T}^{\boldsymbol{\alpha}_{i}}$ and $z_{i}$ by $\mathbb{T}^{-\boldsymbol{\alpha}_{i}}$, we have $f_{1}\left(\mathbb{T}^{\mathbb{Q}_{1}}, \ldots, \mathbb{T}^{\boldsymbol{\alpha}}{ }_{n}\right)=0$, which is equivalent to $f_{1} \in \mathcal{I}_{\mathbb{Q} \text {. }}$. Then $\prod_{i=1}^{n} y_{i}^{t_{i}} f \in \widetilde{\mathcal{I}}_{\mathbb{Q}}$ and hence $\prod_{i=1}^{n} z_{i}^{t_{i}} y_{i}^{t_{i}} f=\left(z_{i} y_{i}\right)^{t_{i}} f=f+f_{0} \in \widetilde{\mathcal{I}}_{\mathbb{Q}}$, where $f_{0} \in \mathcal{I}_{0}$. Thus $f \in \widetilde{\mathcal{I}}_{\mathbb{Q} \text {. }}$.

Corollary 6.22 Use the notations in Lemma 6.21. $T_{\mathbb{Q}}^{*}$ is isomorphic to $\operatorname{Spec}^{\sigma}\left(\mathbb{Q}\left\{\mathbb{Y}^{ \pm}\right\} / \mathcal{I}_{\mathbb{Q}}\right)$.

Proof: The isomorphism is given by $y_{i} \Rightarrow y_{i}$ and $z_{i} \Rightarrow y_{i}^{-1}$ for $i=1, \ldots, n$.

Corollary 6.23 Let $T_{\mathbb{Q}}^{*}$ and $\mathbf{X}_{\mathbb{Q}}$ be the $\sigma$-torus and the toric $\sigma$-variety defined by $\mathbb{\alpha}$, respectively. Then $T_{\mathbb{Q}}^{*}=\mathbf{X}_{\mathbb{Q}} \cap\left(\mathbb{A}^{*}\right)^{n}$. Equivalently, $T_{\mathbb{Q}}^{*}$ is a Cohn open set in $\mathbf{X}_{\mathbb{Q}}$. 
Proof: From Lemma 6.21 and the fact $\mathcal{I}_{\mathbb{Q}}=\mathbb{I}\left(\mathbf{X}_{\mathbb{Q}}\right)$, we have $T_{\mathbb{Q}}^{*}=\mathbf{X}_{\mathbb{Q}} \cap\left(\mathbb{A}^{*}\right)^{n}$. Then $\mathbf{X}_{\mathbb{Q}} \backslash T_{\mathbb{Q}}^{*}=\cup_{i=1}^{n} \mathbb{V}\left(\left\{\mathcal{I}_{\mathbb{Q}}, y_{i}\right\}\right)$. Since $\mathcal{I}_{\mathbb{Q}}$ is a reflexive and prime $\sigma$-ideal not containing any $y_{i}$, $\mathbb{V}\left(\left\{\mathcal{I}_{\mathbb{Q}}, y_{i}\right\}\right)$ is a proper sub- $\sigma$-variety of $\mathbf{X}_{\mathbb{Q}}$, or equivalently, $T_{\mathbb{Q}}^{*}$ is an open set of $\mathbf{X}_{\mathbb{Q}}$.

Corollary 6.24 A $\sigma$-torus $T^{*}$ is a toric $\sigma$-variety and has a group structure. As a consequence, $T^{*}=t \cdot T^{*}$ for any $t \in \mathbb{T}^{*}$.

Proof: From Lemma 6.21, it suffices to prove the corollary for $\widetilde{T}_{\mathbb{Q}}^{*}=\mathbb{V}\left(\left\{\widetilde{\mathcal{I}}_{\mathbb{Q}}\right\}\right)$. By Theorem 6.11, $\mathcal{I}_{\mathbb{Q}} \subset \mathbb{Q}\{\mathbb{Y}\}$ is toric. Then, it is apparent that $\widetilde{\mathcal{I}}_{\mathbb{Q}}=\left[\mathcal{I}_{\mathbb{Q}}, y_{1} z_{1}-1, \ldots, y_{n} z_{n}-1\right]$ is also toric. To show that $\widetilde{T}_{\mathbb{Q}}^{*}$ has a group structure, let $a, b \in \widetilde{T}_{\mathbb{Q}}^{*}$. Since $y_{i} z_{i}-1 \in \mathbb{I}\left(\widetilde{T}_{\mathbb{Q}}^{*}\right), i=1, \ldots, n$, $a^{-1}$ is well defined. Then for each $\sigma$-binomial $\mathbb{Y}^{\mathbf{f}}-\mathbb{Y}^{\mathbf{g}} \in \mathbb{I}\left(\widetilde{T}_{\mathbb{Q}}^{*}\right)$, we have $\left(\mathbb{Y}^{\mathbf{f}}-\mathbb{Y}^{\mathbf{g}}\right)(a)=$ $0,\left(\mathbb{Y}^{\mathbf{f}}-\mathbb{Y}^{\mathbf{g}}\right)(b)=0$. Then $\left(\mathbb{Y}^{\mathbf{f}}-\mathbb{Y}^{\mathbf{g}}\right)(a b)=0$ and $\left(\mathbb{Y}^{\mathbf{f}}-\mathbb{Y}^{\mathbf{g}}\right)\left(a^{-1}\right)=0$. It implies that the $\sigma$-torus is a group. Since $\widetilde{T}_{\mathbb{Q}}^{*}$ is a group, for $t \in \widetilde{T}_{\mathbb{Q}}^{*}$, we have $t \cdot \widetilde{T}_{\mathbb{Q}}^{*} \subset \widetilde{T}_{\mathbb{Q}}^{*}$ and $t^{-1} \cdot \widetilde{T}_{\mathbb{Q}}^{*} \subset \widetilde{T}_{\mathbb{Q}}^{*}$ which implies $t \cdot \widetilde{T}_{\mathbb{\alpha}}^{*}=\widetilde{T}_{\mathbb{Q}}^{*}$.

Similar to Theorem 6.9, we have the following result.

Theorem 6.25 $T^{*}$ is a $\sigma$-torus if and only if there exists a $\mathbb{Z}[x]$-lattice $L$ in $\mathbb{Z}[x]^{m}$ such that $T^{*} \cong \operatorname{Spec}^{\sigma}(\mathbb{Q}\{L\})$, where

$$
\mathbb{Q}\{L\}=\left\{\sum_{\boldsymbol{\beta} \in L} a_{\boldsymbol{\beta}} \mathbb{T}^{\boldsymbol{\beta}} \mid a_{\boldsymbol{\beta}} \in \mathbb{Q}, a_{\boldsymbol{\beta}} \neq 0 \text { for finitely many } \boldsymbol{\beta}\right\} .
$$

Proof: Let $T_{\mathbb{Q}}$ be the quasi $\sigma$-torus defined by $\mathbb{\alpha}, T_{\mathbb{Q}}^{*}$ the Cohn $*$-closure of $T_{\mathbb{Q}}$ in $\left(\mathbb{A}^{*}\right)^{n}$, $L$ the $\mathbb{Z}[x]$-lattice generated by $\mathbb{\alpha}$, and $\mathcal{I}_{\mathbb{Q}}$ defined in (37). For a set of $\sigma$-indeterminates $\mathbb{Z}=\left\{z_{1}, \ldots, z_{n}\right\}$, define the following morphism of $\sigma$-rings

$$
\theta: \mathbb{Q}\{\mathbb{Y}, \mathbb{Z}\} \longrightarrow \mathbb{Q}\{L\}, \text { where } \theta\left(y_{i}\right)=\mathbb{T}^{\boldsymbol{\alpha}_{i}}, \theta\left(z_{i}\right)=\mathbb{T}^{-\boldsymbol{\alpha}_{i}}, i=1, \ldots, n
$$

It is easy to check that the map $\theta$ is surjective. By Theorem 6.21, $\widetilde{\mathcal{I}}_{\mathbb{Q}}=\operatorname{ker}(\theta)=\left[\mathcal{I}_{\alpha}, y_{1} z_{1}-\right.$ $\left.1, \ldots, y_{n} z_{n}-1\right]$ which is the defining ideal for $\widetilde{T}_{\mathbb{Q}}^{*}$. Since $\widetilde{T}_{\mathbb{Q}}^{*}$ is isomorphic to $T_{\mathbb{Q}}^{*}$, we have $\mathbb{Q}\{\mathbb{Y}, \mathbb{Z}\} / \widetilde{\mathcal{I}}_{\mathbb{Q}} \simeq \mathbb{Q}\{L\}$ and $T_{\mathbb{Q}}^{*} \cong \operatorname{Spec}^{\sigma}(\mathbb{Q}\{L\})$. We thus proved that a $\sigma$-torus is isomorphic to $\operatorname{Spec}^{\sigma}(\mathbb{Q}\{L\})$.

If $T^{*}=\operatorname{Spec}^{\sigma}(\mathbb{Q}\{L\})$, where $L=\mathbb{Z}[x]\left(\boldsymbol{\alpha}_{1}, \ldots, \boldsymbol{\alpha}_{n}\right)$ is a $\mathbb{Z}[x]$-lattice for $\boldsymbol{\alpha}_{i} \in \mathbb{Z}[x]^{m}$. Let $T_{\mathbb{Q}}^{*}$ be the $\sigma$-torus defined by $\mathbb{\alpha}=\left\{\boldsymbol{\alpha}_{1}, \ldots, \boldsymbol{\alpha}_{n}\right\}$. Then as we just proved, the coordinate ring of $T_{\mathbb{Q}}^{*}$ is isomorphic to $\mathbb{Q}\{L\}$. Then $T^{*}$ is isomorphic to $T_{\mathbb{Q}}^{*}$.

As a consequence, we can prove that the tori defined by two sets of generators of the same $\mathbb{Z}[x]$-lattice are isomorphic.

Corollary 6.26 Let $\mathbb{Q}=\left\{\boldsymbol{\alpha}_{1}, \ldots, \boldsymbol{\alpha}_{n}\right\}$ and $\boldsymbol{\beta}=\left\{\boldsymbol{\beta}_{1}, \ldots, \boldsymbol{\beta}_{s}\right\}$ be two sets of generators for the same $\mathbb{Z}[x]$-lattice. Then $T_{\mathbb{Q}}^{*}$ and $T_{\mathbb{\beta}}^{*}$ are isomorphic as $\sigma$-varieties and groups.

Proof: By Theorem 6.25 , it suffices to show that $\mathbb{Q}\{\mathbb{Z}[x] \propto\}$ is isomorphic to $\mathbb{Q}\{\mathbb{Z}[x] \mathbb{B}\}$ as $\sigma$-rings. Use notations introduced in the proof of Lemma 6.6. Define

$$
\phi: \mathbb{Q}\{\mathbb{Z}[x] \alpha\} \Rightarrow \mathbb{Q}\{\mathbb{Z}[x] \mathbb{B}\} \text { and } \theta: \mathbb{Q}\{\mathbb{Z}[x] \mathbb{B}\} \Rightarrow \mathbb{Q}\{\mathbb{Z}[x] \propto\}
$$


by setting $\phi\left(\mathbb{T}_{i}^{\mathbb{Q}}\right)=\prod_{k=1}^{s} \mathbb{T}^{m_{k i} \beta_{k}}$ and $\theta\left(\mathbb{T}_{j}^{\boldsymbol{\beta}}\right)=\prod_{k=1}^{n} \mathbb{T}^{n_{k i} \mathbb{Q}_{k}}$. It is clear that $\phi$ and $\theta$ are $\sigma$-morphisms and group homomorphisms. Since $A=A N M$ and $B=B M N$, it can be checked that $\phi \circ \theta=i d$ and $\theta \circ \phi=i d$. Then, $\mathbb{Q}\{\mathbb{Z}[x] \mathbb{Q}\}$ is isomorphic to $\mathbb{Q}\{\mathbb{Z}[x] \mathbb{B}\}$ and hence $T_{\mathbb{Q}}^{*}$ and $T_{\mathbb{\beta}}^{*}$ are isomorphic as $\sigma$-varieties.

Since $T_{\mathbb{Q}}^{*}$ is Cohn open in $\mathbf{X}_{\mathbb{Q}}$, as a consequence of Corollary 6.26, we have

Corollary 6.27 Let $\mathbb{\alpha}=\left\{\boldsymbol{\alpha}_{1}, \ldots, \boldsymbol{\alpha}_{n}\right\}$ and $\boldsymbol{\beta}=\left\{\boldsymbol{\beta}_{1}, \ldots, \boldsymbol{\beta}_{s}\right\}$ be two sets of generators for the same $\mathbb{Z}[x]$-lattice. Then $\mathbf{X}_{\mathbb{Q}}$ and $\mathbf{X}_{\beta}$ are birationally equivalent $\sigma$-varieties.

Example 6.28 Let $N_{1}=\left[\begin{array}{lll}x & 1 & x \\ x-1 & 1 & x+1\end{array}\right], N_{2}=\left[\begin{array}{lll}0 & 1 & 0 \\ -1 & 1 & 1\end{array}\right]$, and $N_{3}=\left[\begin{array}{lll}0 & 1 & 0 \\ 0 & 0 & 1\end{array}\right]$. Then $\left(N_{1}\right)=\left(N_{2}\right)=\left(N_{3}\right)$ and the corresponding toric $\sigma$-ideals are $\mathcal{I}_{1}=\left[y_{1} y_{3}-y_{2}^{2 x}\right]$, $\mathcal{I}_{2}=\left[y_{1} y_{3}-1\right]$, and $\mathcal{I}_{3}=\left[y_{1}-1\right]$, respectively. Then, $\mathbb{V}\left(\mathcal{I}_{i}\right), i=1,2,3$ are isomorphic as tori in $\left(\mathbb{A}^{*}\right)^{3}$ and birationally equivalent as $\sigma$-varieties in $\mathbb{A}^{3}$.

An algebraic torus is isomorphic to $\left(\mathbb{C}^{*}\right)^{m}$ for some $m \in \mathbb{N}[5]$. The following example shows that this is not valid in the difference case.

Example 6.29 Let $\boldsymbol{\alpha}_{1}=(2), \boldsymbol{\alpha}_{2}=(x)$, and $\boldsymbol{\alpha}=\left\{\boldsymbol{\alpha}_{1}, \boldsymbol{\alpha}_{2}\right\}$. Then $T_{\mathbb{Q}}^{*}=\mathbb{V}\left(\left\{y_{1}^{x}-y_{2}^{2}\right\}\right)$. We claim that $T_{\mathbb{Q}}^{*}$ is not isomorphic $\mathbb{A}^{*}$. By Theorem [6.25, we need to show $\mathcal{E}_{1}=\mathbb{Q}\left\{t, t^{-1}\right\}$ is not isomorphic to $\mathcal{E}_{2}=\mathbb{Q}\left\{s^{2}, s^{-2}, s^{x}, s^{-x}\right\}$, where $t$ and $s$ are $\sigma$-indeterminates. Suppose the contrary, there is an isomorphism $\theta: \mathcal{E}_{1} \Rightarrow \mathcal{E}_{2}$ and $\theta(t)=p(s) \in \mathcal{E}_{2}$. Then there exists a $q(z) \in \mathbb{Q}\{z\}$ such that $s^{2}=q(p(s))$ which is possible only if $q=z, p=s^{2}$. Since $s^{x} \in \mathcal{E}_{2}$, there exists an $r(z) \in \mathbb{Q}\{z\}$ such that $s^{x}=r\left(s^{2}\right)$ which is impossible.

Let $T^{*} \subseteq\left(\mathbb{A}^{*}\right)^{n}$ be a $\sigma$-torus and $X \subseteq \mathbb{A}^{n}$ a $\sigma$-variety. $T^{*}$ is said to act on $X$ if there exists a $\varphi \in \mathbb{Q}\left\{y_{1}, \ldots, y_{n}, z_{1}, \ldots, z_{n}\right\}$ such that for all $\mathcal{E} \in \mathscr{E}_{\mathcal{F}}$,

$$
\varphi: T^{*}(\mathcal{E}) \times X(\mathcal{E}) \longrightarrow X(\mathcal{E})
$$

is a $\sigma$-morphism as well as a group action of $T^{*}(\mathcal{E})$ on $X(\mathcal{E})$, that is, $\varphi(\mathbf{1}, \mathbf{h})=\mathbf{h}$ and $\varphi\left(\boldsymbol{\epsilon}_{1} \cdot \boldsymbol{\epsilon}_{2}, \mathbf{h}\right)=\varphi\left(\boldsymbol{\epsilon}_{1}, \varphi\left(\boldsymbol{\epsilon}_{2}, \mathbf{h}\right)\right)$.

The following result gives a new characterization of toric $\sigma$-varieties.

Theorem 6.30 A $\sigma$-variety $X$ is toric if and only if $X$ contains a $\sigma$-torus $T^{*}$ as an open subset and with a group action of $T^{*}$ on $X$ extending the natural group action of $T^{*}$ on itself.

Proof: " $\Rightarrow$ " For $\propto$ given in (35), let $X=\mathbf{X}_{\mathbb{Q}}$ be the toric $\sigma$-variety defined by $\propto$ and $T_{\mathbb{Q}}^{*}$ the $\sigma$-torus defined by $\mathbb{\alpha}$. By Corollary 6.23, $T_{\mathbb{Q}}^{*}$ is open in $X$. The group action of $T_{\mathbb{Q}}^{*}$ on itself can be extended to $\mathbb{A}^{n}: T_{\mathbb{Q}}^{*} \times \mathbb{A}^{n} \longrightarrow \mathbb{A}^{n}$ by $\left(a_{1}, \ldots, a_{n}\right) \cdot\left(t_{1}, \ldots, t_{n}\right)=\left(a_{1} t_{1}, \ldots, a_{n} t_{n}\right)$. By Corollary 6.24, for $t \in T_{\mathbb{Q}}^{*}, T_{\mathbb{Q}}^{*}=t \cdot T_{\mathbb{Q}}^{*} \subseteq t \cdot X$ and $t \cdot X$ is an irreducible $\sigma$-variety whose defining $\sigma$-ideal is $\mathcal{I}_{t}=\{f \in \mathbb{Q}\{\mathbb{Y}\} \mid f(t \mathbb{Y}) \in \mathbb{I}(X)\}$. Since $X$ is the Cohn closure of $T_{\mathbb{Q}} \subset T_{\mathbb{Q}}^{*}$ in $\mathbb{A}^{n}$, we have $X \subseteq t \cdot X$. Set $t:=t^{-1}$, we have $X=t \cdot X$. So $T_{\mathbb{Q}}^{*} \times T_{\mathbb{Q}}^{*} \longrightarrow T_{\mathbb{Q}}^{*}$ can be extended to $T_{\mathbb{Q}}^{*} \times X \longrightarrow X$. 
" $\Leftarrow$ " Let $X$ be a $\sigma$-variety containing a $\sigma$-torus $T^{*}$ which is isomorphic to $T_{\boldsymbol{\alpha}}^{*}$ for $\mathbb{\alpha}$ defined in (35). Then, we have the following commutative diagram:

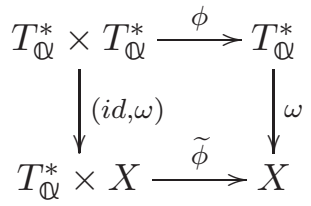

where $\phi$ is the group operation of $T_{\mathbb{Q}}^{*}, \widetilde{\phi}$ is the extension of $\phi$ to $T^{*} \times X$, and $\omega$ is the inclusion map of $T_{\mathbb{Q}}^{*}$ into $\mathbb{X}$.

Since $T_{\mathbb{Q}}^{*}$ is a $\sigma$-torus, by Theorem 6.25, there exists a $\mathbb{Z}[x]$-lattice $L=(\boldsymbol{\alpha})$ in $\mathbb{Z}[x]^{m}$ such that $T_{\mathbb{Q}}^{*} \cong \operatorname{Spec}^{\sigma}(\mathbb{Q}\{L\})$. Let $\Theta(X)$ be the set of all the $\sigma$-polynomial functions on $X$ and $\Theta(X) \cong \mathbb{Q}\{X\}$. From (40), we obtain the following commutative diagram

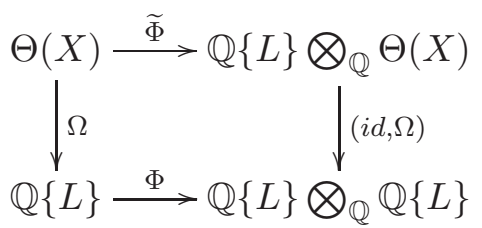

Since $T_{\mathbb{Q}}^{*}$ is an open set in $X, \Omega: \Theta(X) \Rightarrow \mathbb{Q}\{L\}$ is the inclusion map of $\Theta(X)$ into $\mathbb{Q}\{L\}$. Therefore, each element $p \in \Theta(X)$ can be written as $p=\sum_{\boldsymbol{\beta} \in L} a_{\boldsymbol{\beta}} \mathbb{T}^{\boldsymbol{\beta}}$, where $a_{\boldsymbol{\beta}}$ is zero except a finite number of $\boldsymbol{\beta}$. From the morphism $\Phi$ in the diagram, we have $\Phi(p)=\sum_{\boldsymbol{\beta} \in L} a_{\boldsymbol{\beta}} \mathbb{T}^{\boldsymbol{\beta}} \otimes \mathbb{T}^{\boldsymbol{\beta}} \in \mathbb{Q}\{L\} \bigotimes_{\mathbb{Q}} \mathbb{Q}\{L\}$. From the injective morphism $(i d, \Omega)$ in the diagram, we have $\bar{p}=(i d, \Omega)(\widetilde{\Phi}(p))=\Phi(p)=\sum_{\boldsymbol{\beta} \in L} a_{\boldsymbol{\beta}} \mathbb{T}^{\boldsymbol{\beta}} \otimes \mathbb{T}^{\boldsymbol{\beta}} \in \mathbb{Q}\{L\} \bigotimes_{\mathbb{Q}} \Theta(X)$. This is possible only if $\mathbb{T}^{\boldsymbol{\beta}} \in \Theta(X)$ for each $a_{\boldsymbol{\beta}} \mathbb{T}^{\boldsymbol{\beta}} \in L$. For otherwise, $\bar{p}$ should contain a term of the

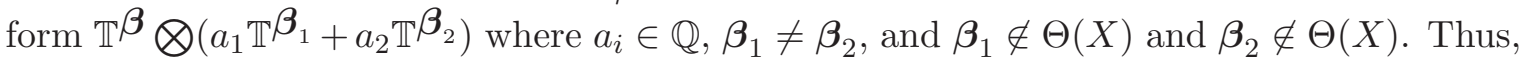
$\bar{p}$ contains the terms $a_{1} \mathbb{T}^{\boldsymbol{\beta}} \otimes \mathbb{T}^{\boldsymbol{\beta}_{1}}+a_{2} \mathbb{T}^{\boldsymbol{\beta}} \otimes \mathbb{T}^{\boldsymbol{\beta}_{2}}$, which implies $\boldsymbol{\beta}=\boldsymbol{\beta}_{1}=\boldsymbol{\beta}_{2}$, a contradiction. Therefore, there exists an affine $\mathbb{N}[x]$-module $S \subseteq L$ such that $\Theta(X)=\bigoplus_{\boldsymbol{\beta} \in S} b_{\boldsymbol{\beta}} \mathbb{T}^{\boldsymbol{\beta}}$. Since $\mathbb{Z}[x]^{n}$ is Notherian, there exists a finite set $\gamma \subseteq L$ such that $S=\mathbb{N}[x] \gamma$ is an affine $\mathbb{N}[x]$-module and $\Theta(X) \cong \mathbb{Q}\{S\}$. Thus $X=\operatorname{Spec}^{\sigma}(\mathbb{Q}\{S\})$.

Following Theorems 6.9, 6.11, and 6.30, we have Theorem 1.3 ,

\section{4 $\sigma$-Chow form and order of toric $\sigma$-variety}

In this section, we show that the $\sigma$-Chow form [24, 9] of a toric $\sigma$-variety $\mathbf{X}_{\mathbb{Q}}$ is the sparse $\sigma$-resultant [21] with support $\mathbb{Q}$. As a consequence, we can give a bound for the order of $\mathbf{X}_{\mathbb{Q}}$.

Let $\mathbb{Q}=\left\{\boldsymbol{\alpha}_{1}, \ldots, \boldsymbol{\alpha}_{n}\right\}$ be a subset of $\mathbb{Z}[x]^{m}$ and $\mathbf{X}_{\mathbb{Q}}$ the toric $\sigma$-variety defined by $\mathbb{\alpha}$. In order to establish a connection between the $\sigma$-Chow form of $\mathbf{X}_{\mathbb{Q}}$ and the $\sigma$-sparse resultant with support $\mathbb{\alpha}$, we assume that $\mathbb{\alpha}$ is Laurent transformally essential [21], that is

$$
\operatorname{rk}\left(M_{L}\right)=m
$$


where $M_{L}$ is the matrix with $\boldsymbol{\alpha}_{i}$ as the $i$-th column. Let $\mathbb{T}=\left(t_{1}, \ldots, t_{m}\right)$ be a set of $\sigma$ indeterminates. Here, $\mathbb{\alpha}$ is Laurent transformally essential means that there exist indices

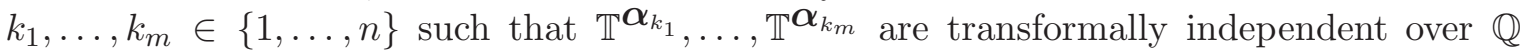
[21].

Let $\mathcal{A}=\left\{M_{1}=\mathbb{T}^{\boldsymbol{\alpha}_{1}}, \ldots, M_{n}=\mathbb{Y}^{\boldsymbol{\alpha}_{n}}\right\}$ and

$$
\mathbb{P}_{i}=u_{i 0}+u_{i 1} M_{1}+\cdots+u_{i n} M_{n}(i=0, \ldots, m)
$$

$m+1$ generic Laurent $\sigma$-polynomials with the same support $\mathbb{\alpha}$. Denote $\mathbf{u}_{i}=\left(u_{i 0}, \ldots, u_{i n}\right)$, $i=0, \ldots, m$. Since $\mathcal{A}$ is Laurent transformally essential, the $\sigma$-sparse resultant of $\mathbb{P}_{0}, \mathbb{P}_{1}, \ldots$, $\mathbb{P}_{m}$ exists [21], which is denoted by $R_{\mathbb{Q}} \in \mathbb{Q}\left\{\mathbf{u}_{0}, \ldots, \mathbf{u}_{m}\right\}$.

By Lemma 6.2. $\mathbf{X}_{\mathbb{Q}} \subset \mathbb{A}^{n}$ is an irreducible $\sigma$-variety of dimension $\operatorname{rk}\left(M_{L}\right)=m$. Then, the $\sigma$-Chow form of $\mathbf{X}_{\mathbb{Q}}$, denoted by $C_{\mathbb{Q}} \in \mathbb{Q}\left\{\mathbf{u}_{0}, \ldots, \mathbf{u}_{m}\right\}$, can be obtained by intersecting $\mathbf{X}_{\mathbb{Q}}$ with the following generic $\sigma$-hyperplanes [24]

$$
\mathbb{L}_{i}=u_{i 0}+u_{i 1} y_{1}+\cdots+u_{i n} y_{n}(i=0, \ldots, m) .
$$

We have

Theorem 6.31 Up to a sign, the sparse $\sigma$-resultant $R_{\mathbb{Q}}$ of $\mathbb{P}_{i}(i=0, \ldots, m)$ is the same as the $\sigma$-Chow form $C_{\mathbb{Q}}$ of $\mathbf{X}_{\mathbb{Q}}$ w.r.t. the generic hyperplanes $\mathbb{L}_{i}(i=0, \ldots, m)$.

Proof: Let $\left[\mathbb{P}_{0}, \ldots, \mathbb{P}_{m}\right]$ be the $\sigma$-ideal in $\mathbb{Q}\left\{\mathbf{u}_{0}, \ldots, \mathbf{u}_{m}, \mathbb{T}^{ \pm}\right\}$. From [21],

$$
\left[\mathbb{P}_{0}, \mathbb{P}_{1}, \ldots, \mathbb{P}_{m}\right] \cap \mathbb{Q}\left\{\mathbf{u}_{0}, \ldots, \mathbf{u}_{m}\right\}=\operatorname{sat}\left(R_{\mathbb{Q}}, R_{1}, \ldots, R_{l}\right)
$$

is a reflexive prime $\sigma$-ideal of codimension one in $\mathbb{Q}\left\{\mathbf{u}_{0}, \ldots, \mathbf{u}_{m}\right\}$. Let $\mathcal{I}_{\mathbb{Q}}=\mathbb{I}\left(\mathbf{X}_{\boldsymbol{\alpha}}\right)$ and $\left[\mathcal{I}_{\mathbb{Q}}, \mathbb{L}_{0}, \mathbb{L}_{1}, \ldots, \mathbb{L}_{m}\right]$ be generated in $\mathbb{Q}\left\{\mathbb{Y}, \mathbf{u}_{0}, \ldots, \mathbf{u}_{m}\right\}$. From $[24$,

$$
\left[\mathcal{I}_{\mathbb{Q}}, \mathbb{L}_{0}, \mathbb{L}_{1}, \ldots, \mathbb{L}_{m}\right] \cap \mathbb{Q}\left\{\mathbf{u}_{0}, \ldots, \mathbf{u}_{m}\right\}=\operatorname{sat}\left(C_{\mathbb{Q}}, C_{1}, \ldots, C_{t}\right)
$$

is a reflexive prime $\sigma$-ideal of codimension one in $\mathbb{Q}\left\{\mathbf{u}_{0}, \ldots, \mathbf{u}_{m}\right\}$. Then, it suffices to show

$$
\left[\mathbb{P}_{0}, \mathbb{P}_{1}, \ldots, \mathbb{P}_{m}\right] \cap \mathbb{Q}\left\{\mathbf{u}_{0}, \ldots, \mathbf{u}_{m}\right\}=\left[\mathcal{I}_{\mathbb{Q}}, \mathbb{L}_{0}, \mathbb{L}_{1}, \ldots, \mathbb{L}_{m}\right] \cap \mathbb{Q}\left\{\mathbf{u}_{0}, \ldots, \mathbf{u}_{m}\right\} .
$$

Let $\mathcal{I}_{\mathbb{T}}=\left[y_{1}-M_{1}, \ldots, y_{n}-M_{n}\right]$ be the $\sigma$-ideal generated by $y_{i}-M_{i}, i=1, \ldots, n$ in $\mathbb{Q}\left\{\mathbb{Y}, \mathbb{T}^{ \pm}\right\}$. By (38), $\mathcal{I}_{\mathbb{Q}}=\mathcal{I}_{\mathbb{T}} \cap \mathbb{Q}\{\mathbb{Y}\}$. Then, $\left[\mathcal{I}_{\mathbb{Q}}, \mathbb{L}_{0}, \ldots, \mathbb{L}_{m}\right] \cap \mathbb{Q}\left\{\mathbf{u}_{0}, \ldots, \mathbf{u}_{m}\right\}=\left[y_{1}-M_{1}, \ldots, y_{n}-\right.$ $\left.M_{n}, \mathbb{L}_{0}, \ldots, \mathbb{L}_{m}\right] \cap \mathbb{Q}\left\{\mathbf{u}_{0}, \ldots, \mathbf{u}_{m}\right\}=\left[y_{1}-M_{1}, \ldots, y_{n}-M_{n}, \mathbb{P}_{0}, \ldots, \mathbb{P}_{m}\right] \cap \mathbb{Q}\left\{\mathbf{u}_{0}, \ldots, \mathbf{u}_{m}\right\}$. Since $\mathbb{P}_{i} \in \mathbb{Q}\left\{\mathbf{u}_{0}, \ldots, \mathbf{u}_{n}, \mathbb{T}^{ \pm}\right\}$does not contain any $y_{i}^{x^{j}}$ in $\Theta(\mathbb{Y})$, we have $\left[y_{1}-M_{1}, \ldots, y_{n}-\right.$ $\left.M_{n}, \mathbb{P}_{0}, \ldots, \mathbb{P}_{m}\right] \cap \mathbb{Q}\left\{\mathbf{u}_{0}, \ldots, \mathbf{u}_{m}\right\}=\left[\mathbb{P}_{0}, \ldots, \mathbb{P}_{m}\right] \cap \mathbb{Q}\left\{\mathbf{u}_{0}, \ldots, \mathbf{u}_{m}\right\}$, and the theorem is proved.

To give a bound for the order of $\mathbf{X}_{\mathbb{Q}}$, we need to introduce the concept of Jacobi number. Let $M=\left(m_{i j}\right)$ be an $m \times m$ matrix with elements either in $\mathbb{N}$ or $-\infty$. A diagonal sum of $M$ is any sum $m_{1 \sigma(1)}+m_{2 \sigma(2)}+\cdots+m_{m \sigma(m)}$ with $\sigma$ a permutation of $1, \ldots, m$. The Jacobi number of $M$ is the maximal diagonal sum of $M$, denoted by $\operatorname{Jac}(M)$ [21].

Let $\mathbb{Q}=\left\{\mathbb{\alpha}_{1}, \ldots, \mathbb{Q}_{n}\right\} \subset \mathbb{Z}[x]^{m}$ and $A=\left(a_{i j}\right)_{m \times n}$ the matrix with $\boldsymbol{\alpha}_{i}$ as the $i$-th column. For each $i \in\{1, \ldots, m\}$, let $o_{i}=\max _{k=1}^{n} \operatorname{deg}\left(a_{i k}, x\right)$ and assume that $\operatorname{deg}(0, x)=-\infty$. Since $A$ does not contain zero rows, no $a_{i j}$ is $-\infty$. For a $p(x) \in \mathbb{Z}[x]$, let $\operatorname{deg}(p, x)=$ $\min \left\{k \in \mathbb{N} \mid\right.$ s.t. $\left.\operatorname{coeff}\left(p, x^{k}\right) \neq 0\right\}$ and $\operatorname{deg}(0, x)=0$. For each $i \in\{1, \ldots, m\}$, let $\underline{o}_{i}=$ $\min _{k=1}^{n} \underline{\operatorname{deg}}\left(a_{i k}, x\right)$ and $\underline{o}=\sum_{i=1}^{m} \underline{o}_{i}$. Then we have 
Theorem 6.32 Use the notations just introduced. Let $\mathbf{X}_{\mathbb{Q}}$ be the toric variety defined by $\mathbb{\alpha}$. Then $\operatorname{ord}\left(\mathbf{X}_{\mathbb{Q}}\right) \leq \sum_{i=1}^{m}\left(o_{i}-\underline{o}_{i}\right)$.

Proof: Use the notations in Theorem 6.31. Since $\mathbb{P}_{i}$ in (41) have the same support for all $i, \operatorname{ord}\left(R_{\mathbb{Q}}, \mathbf{u}_{i}\right)$ are the same for all $i$. The order matrix for $\mathbb{P}_{i}$ given in (41) is $O=$ $\left(\operatorname{ord}\left(\mathbb{P}_{i}, t_{j}\right)\right)_{(m+1) \times m}=\left(o_{i j}\right)_{(m+1) \times m}$, where $o_{i j}=o_{j}$. That is, all rows of $O$ are the same. Let $\bar{O}$ be obtained from $O$ by deleting the any row of $O$. Then $J=\operatorname{Jac}(\bar{O})=\sum_{i=1}^{m} o_{i}$. By Theorem 4.17 of [21], $\operatorname{ord}\left(R_{\mathbb{Q}}, \mathbf{u}_{i}\right) \leq J-\underline{o}=\sum_{i=1}^{m}\left(o_{i}-\underline{o}_{i}\right)$. By Theorem 6.12 of [24], $\operatorname{ord}\left(\mathbf{X}_{\mathbb{Q}}\right)=\operatorname{ord}\left(C_{\mathbb{Q}}, \mathbf{u}_{i}\right)$ for each $i=0, \ldots, m$. By Theorem 6.31, $C_{\mathbb{Q}}=R_{\mathbb{Q}}$. Then the theorem is proved.

\section{$7 \quad$ Algorithms for $\mathbb{Z}[x]$-lattice and binomial $\sigma$-ideal}

In this section, we give algorithms to decide whether a given $\mathbb{Z}[x]$-lattice $L$ is $x$-, $\mathbb{Z}-, M-$, or $\mathbb{Z}[x]$-saturated, and in the negative case to compute the $x_{-}^{-}, \mathbb{Z}-, M_{-}^{-}$, or $\mathbb{Z}[x]$-saturation of $L$. Based on these algorithms, we give algorithms to compute the reflexive closure, the well-mixed closure, the perfect closure of a finitely generated Laurent binomial $\sigma$-ideal and an algorithm to decompose a finitely generated perfect $\sigma$-ideal as the intersection of reflexive prime $\sigma$-ideals.

In this section, the base files $\mathcal{F}$ is assumed to be inversive and algebraically closed, since such conditions are required in Theorems 4.23, 4.29, 4.43, 5.28,

The following well-known algorithms will be used.

- Let $\mathbb{f}$ be a finite set of elements in $\mathbb{Z}[x]^{n}$. We need to compute the reduced Gröbner basis of the $\mathbb{Z}[x]$-module ( $\mathbb{E}$ ) [4, p. 197].

- Let $\mathbb{D}$ be $\mathbb{Z}, \mathbb{Q}[x], \mathbb{Z}_{p}[x]$, or $\mathbb{Q}[x] /(q(x))$, where $q(x)$ is an irreducible polynomial in $\mathbb{Q}[x]$. Then $\mathbb{D}$ is either a PID or a field. For a finite set $S \subset \mathbb{D}^{n}$ and a matrix $M \in \mathbb{D}^{n \times s}$, we need to compute the Hermite normal form of the $\mathbb{D}$-module generated by $S$ and a basis for the $\mathbb{D}$-module: $\operatorname{ker}(M)=\left\{X \in \mathbb{D}^{s} \mid M X=0\right\}$ [2, p.68, p.74].

\section{1 $x$-saturation of $\mathbb{Z}[x]$-lattice}

In this section, we give algorithms to check whether a $\mathbb{Z}[x]$-lattice $L$ is $x$-saturated and in the negative case to compute the $x$-saturation of $L$.

Let $\mathbf{f}_{1}, \ldots, \mathbf{f}_{s} \in \mathbb{Z}[x]^{n}$ and $L=\left(\mathbf{f}_{1}, \ldots, \mathbf{f}_{s}\right)$. If $L$ is not $x$-saturated, then there exist $g_{i} \in \mathbb{Z}[x]$ such that $\sum_{i=1}^{s} g_{i} \mathbf{f}_{i}=x \mathbf{h}$ and $\mathbf{h} \notin L$. Setting $g_{i}(x)=g_{i}(0)+x \widetilde{g}_{i}(x)$ and $\widetilde{\mathbf{h}}=$ $\mathbf{h}-\sum_{i=1}^{s} \widetilde{g}_{i}(x) \mathbf{f}_{i}$, we have

$$
\sum_{i=1}^{s} g_{i}(0) \mathbf{f}_{i}=x \widetilde{\mathbf{h}}
$$

where $\widetilde{\mathbf{h}} \notin L$. Set $x=0$ in the above equation, we have

$$
\sum_{i=1}^{s} g_{i}(0) \mathbf{f}_{i}(0)=0
$$


that is, $G=\left(g_{1}(0), \ldots, g_{s}(0)\right)^{\tau}$ is in the kernel of the matrix $F=\left[\mathbf{f}_{1}(0), \ldots, \mathbf{f}_{s}(0)\right] \in \mathbb{Z}^{n \times s}$, which can be obtained by existing algorithms [2, page 74]. From $G$ and (42), we may compute $\widetilde{\mathbf{h}}$. This observation leads to the following algorithm.

\section{$\operatorname{Algorithm~} 1-\operatorname{XFACTOR}\left(\mathbf{f}_{1}, \ldots, f_{s}\right)$}

Input: A generalized Hermite normal form $\left\{\mathbf{f}_{1}, \ldots, \mathbf{f}_{s}\right\} \subset \mathbb{Z}[x]^{n}$.

Output: $\emptyset$, if $L=\left(\mathbf{f}_{1}, \ldots, \mathbf{f}_{s}\right)$ is $x$-saturated; otherwise, a finite set $\left\{\left(\mathbf{h}_{i}, \mathbf{e}_{i}\right) \mid i=1, \ldots, r\right\}$ such that $\mathbf{e}_{i}=\left(e_{i 1}, \ldots, e_{i s}\right)^{\tau} \in \mathbb{Z}^{s}, \mathbf{h}_{i} \notin L$, and $x \mathbf{h}_{i}=\sum_{l=1}^{s} e_{i l} \mathbf{f}_{l} \in L, i=$ $1, \ldots, r$.

1. Set $F=\left[\mathbf{f}_{1}(0), \ldots, \mathbf{f}_{s}(0)\right] \in \mathbb{Z}^{n \times s}$.

2. Compute a basis $E \subset \mathbb{Z}^{s}$ of the $\mathbb{Z}$-module $\operatorname{ker}(F)$ with the algorithm in [2, page 74].

3. Set $H=\emptyset$.

4. While $E \neq \emptyset$

4.1. Let $\mathbf{e}=\left(e_{1}, \ldots, e_{s}\right)^{\tau} \in E$ and $E=E \backslash\{\mathbf{g}\}$.

4.2. Let $\mathbf{h}=\left(e_{1} \mathbf{f}_{1}+\cdots+e_{s} \mathbf{f}_{s}\right) / x$.

4.3. If $\operatorname{grem}\left(\mathbf{h},\left\{\mathbf{f}_{1}, \ldots, \mathbf{f}_{s}\right\}\right) \neq 0$, then add $(\mathbf{h}, \mathbf{e})$ to $H$.

5. Return $H$.

We now give the algorithm to compute the $x$-saturation of a $\mathbb{Z}[x]$-lattice.

Algorithm $2-\operatorname{SATX}\left(\mathbf{f}_{1}, \ldots, \mathbf{f}_{s}\right)$

Input: $\quad$ A finite set $\mathbb{f}=\left\{\mathbf{f}_{1}, \ldots, \mathbf{f}_{s}\right\} \subset \mathbb{Z}[x]^{n}$.

Output: A set of generators of $\operatorname{sat}_{x}\left(\mathbf{f}_{1}, \ldots, \mathbf{f}_{s}\right)$.

1. Compute the generalized Hermite normal form $\mathfrak{g}$ of $\mathbb{f}$.

2. Set $H=\operatorname{XFACTOR}(\mathrm{g})$.

3. If $H=\emptyset$, then output $\mathrm{g}$; otherwise set $\mathbb{f}=\mathrm{g} \cup\{\mathbf{h} \mid(\mathbf{h}, \mathbf{f}) \in H\}$ and goto step 1 .

Example 7.1 Let $\mathcal{C}$ be the following generalized Hermite normal form.

$$
\mathcal{C}=\left[\mathbf{f}_{1}, \mathbf{f}_{2}, \mathbf{f}_{3}\right]=\left[\begin{array}{lll}
-x+2 & 1 & 1 \\
3 x+2 & 1 & 2 x+1 \\
0 & 2 x & x^{2}
\end{array}\right]
$$

In $\operatorname{XFACTOR}(\mathcal{C})$, the kernel of the following matrix

$$
\left[\mathbf{f}_{1}(0), \mathbf{f}_{2}(0), \mathbf{f}_{3}(0)\right]=\left[\begin{array}{lll}
2 & 1 & 1 \\
2 & 1 & 1 \\
0 & 0 & 0
\end{array}\right]
$$

is generated by $\mathbf{e}_{1}=(0,-1,1)^{\tau}$ and $\mathbf{e}_{2}=(1,-2,0)^{\tau}$. In step 4.2 of XFACTOR, we have $\mathcal{C} \mathbf{e}_{1}=-\mathbf{f}_{2}+\mathbf{f}_{3}=\left(0,2 x, x^{2}-2 x\right)^{\tau}=x(0,2, x-2)^{\tau}$. One can check that $(0,2, x-2)^{\tau} \notin(\mathcal{C})$. 
In SATX, computing the generalized Hermite normal form of $\mathcal{C} \cup\left\{(0,2, x-2)^{\tau}\right\}$, we have

$$
\mathcal{C}_{1}=\left[\begin{array}{lll}
-x+2 & 1 & 0 \\
3 x+2 & -3 & 2 \\
0 & 4 & x-2
\end{array}\right]
$$

$\operatorname{XFACTOR}\left(\mathcal{C}_{1}\right)$ returns $\emptyset$. So, $\left(\mathcal{C}_{1}\right)$ is the $x$-saturation of $(C)$.

Theorem 7.2 Algorithms SATX and XFACTOR are correct.

Proof: From the output of Algorithm XFACTOR, in step 3 of SATX, we have $(\mathrm{g}) \subsetneq$ $(\mathrm{g} \cup\{\mathbf{h} \mid(\mathbf{h}, \mathbf{f}) \in H\}) \subseteq \operatorname{sat}_{x}(\mathbb{f})$. Since $\mathbb{Z}[x]^{n}$ is a Noetherian $\mathbb{Z}[x]$-module, SATX will terminate and return the $x$-closure of $(\mathbb{f})$. So, it suffices to show the correctness of Algorithm XFACTOR.

We first explain step 4.2 of Algorithm XFACTOR. Since e $\in \operatorname{ker}(F), \mathbf{h}(0)=\left[\mathbf{f}_{1}(0), \ldots\right.$, $\left.\mathbf{f}_{s}(0)\right] \mathbf{e}=[0, \ldots, 0]^{\tau}$. Therefore, $x$ is a factor of $e_{1} \mathbf{f}_{1}+\cdots+e_{s} \mathbf{f}_{s}$ and thus $\mathbf{h}=\left(e_{1} \mathbf{f}_{1}+\cdots+\right.$ $\left.e_{s} \mathbf{f}_{s}\right) / x \in \mathbb{Z}[x]^{n}$.

To prove the correctness of Algorithm XFACTOR, it suffices to show that $L=\operatorname{sat}_{x}(L)$ if and only if for each $\mathbf{e} \in E, e_{1} \mathbf{f}_{1}+\cdots+e_{s} \mathbf{f}_{s}=x \mathbf{h}$ implies $\mathbf{h} \in L$.

Let $E=\left\{\mathbf{e}_{1}, \ldots, \mathbf{e}_{k}\right\}$ where $\mathbf{e}_{i} \in \mathbb{Z}^{s}$. If $L=\operatorname{sat}_{x}(L)$, then it is clear that $\left(\mathbf{f}_{1}, \ldots, \mathbf{f}_{s}\right) \mathbf{e}_{i}=$ $x \mathbf{h}_{i}$ implies $\mathbf{h}_{i} \in L$. To prove the other direction, let $\left[\mathbf{f}_{1}, \ldots, \mathbf{f}_{s}\right] \mathbf{e}_{i}=x \mathbf{h}_{i}$ for $1 \leq i \leq k$, where $\mathbf{h}_{i} \in L$. Let $x \mathbf{f} \in L$. Then $x \mathbf{f}=\sum_{i=1}^{s} c_{i}(x) \mathbf{f}_{i}$, where $c_{i}(x) \in \mathbb{Z}[x]$. If for each $i, x \mid c_{i}(x)$, then we have $\mathbf{f}=\sum_{i=1}^{s}\left(c_{i}(x) / x\right) \mathbf{f}_{i} \in L$, and the lemma is proved. Otherwise, set $x=0$ in $x \mathbf{f}=\sum_{i=1}^{s} c_{i}(x) \mathbf{f}_{i}$, we obtain $\sum_{i=1}^{s} c_{i}(0) \mathbf{f}_{i}(0)=0$. Hence $Q=\left[c_{1}(0), \ldots, c_{s}(0)\right]^{\tau} \in \operatorname{ker}(F)$ and hence there exist $a_{i} \in \mathbb{Z}, i=1, \ldots, k$ such that $Q=\sum_{i=1}^{k} a_{i} \mathbf{e}_{i}$. Then,

$$
\left[\mathbf{f}_{1}, \ldots, \mathbf{f}_{s}\right] Q=\sum_{i=1}^{k} a_{i}\left[\mathbf{f}_{1}, \ldots, \mathbf{f}_{s}\right] \mathbf{e}_{i}=\sum_{i=1}^{k} a_{i} x \mathbf{h}_{i}=x \widetilde{\mathbf{h}},
$$

where $\widetilde{\mathbf{h}}=\sum_{i=1}^{k} a_{i} \mathbf{h}_{i} \in L$. Then,

$$
\begin{aligned}
x \mathbf{f} & =\sum_{i=1}^{s} c_{i}(x) \mathbf{f}_{i} \\
& =\sum_{i=1}^{s} c_{i}(0) \mathbf{f}_{i}+\sum_{i=1}^{s} x \bar{c}_{i}(x) \mathbf{f}_{i} \\
& =\left[\mathbf{f}_{1}, \ldots, \mathbf{f}_{s}\right] Q+x \sum_{i=1}^{s} \bar{c}_{i}(x) \mathbf{f}_{i} \\
& =x \widetilde{\mathbf{h}}+x \sum_{i=1}^{s} \bar{c}_{i}(x) \mathbf{f}_{i},
\end{aligned}
$$

where $\bar{c}_{i}(x)=\left(c_{i}(x)-c_{i}(0)\right) / x \in \mathbb{Z}[x]$. Hence, $\mathbf{f}=\widetilde{\mathbf{h}}+\sum_{i=1}^{s} \bar{c}_{i}(x) \mathbf{f}_{i} \in L$ and the lemma is proved.

\section{$7.2 \mathbb{Z}$-saturation of $\mathbb{Z}[x]$-lattice}

The key idea to compute $\operatorname{sat}_{\mathbb{Z}}(L)$ for a $\mathbb{Z}[x]$-lattice $L \in \mathbb{Z}[x]^{n}$ is as follows. Let $\mathbb{f}=$ $\left\{\mathbf{f}_{1}, \ldots, \mathbf{f}_{s}\right\}$. Then $(\mathbb{f})$ is not $\mathbb{Z}$-saturated if and only if a linear combination of $\mathbf{f}_{i}$ contains a nontrivial prime factor in $\mathbb{Z}$, that is, $\sum_{i} g_{i} \mathbf{f}_{i}=p \mathbf{f}$, where $p$ is a prime number and $\mathbf{f} \notin(\mathbb{f})$. Furthermore, $\sum_{i} g_{i} \mathbf{f}_{i}=p \mathbf{f}$ with $g_{i} \neq 0 \bmod p$ is valid if and only if $\mathbf{f}_{1}, \ldots, \mathbf{f}_{s}$ are linear dependent over $\mathbb{Z}_{p}[x]$. The fact that $\mathbb{Z}_{p}[x]$ is a PID allows us to compute such linear relations using methods of Hermite normal forms [2]. The following algorithm is based on this observation. 


\section{Algorithm 3-ZFACTOR}

Input: A generalized Hermite normal form $\mathcal{C}=\left\{\mathbf{c}_{1}, \ldots, \mathbf{c}_{s}\right\} \subset \mathbb{Z}[x]^{n}$ given in (4).

Output: $\emptyset$, if $L=(\mathcal{C})$ is $\mathbb{Z}$-saturated; otherwise, a finite set $\left\{\left(\mathbf{h}_{i}, k_{i}, \mathbf{e}_{i}\right) \mid i=1, \ldots, r\right\}$, such that $\mathbf{h}_{i} \in \mathbb{Z}[x]^{n}, k_{i} \in \mathbb{N}, \mathbf{e}_{i}=\left(e_{i 1}, \ldots, e_{i, s}\right)^{\tau} \in \mathbb{Z}[x]^{s}, \mathbf{h}_{i} \notin L$ and $k_{i} \mathbf{h}_{i}=$ $\sum_{l=1}^{s} e_{i l} \mathbf{c}_{l} \in L$ for $i=1, \ldots, r$.

1. Read the numbers $t, r_{i}, l_{i}, c_{r_{i}, 1,0}, i=1, \ldots, t$ from (4).

2. Set $q=\prod_{i=1}^{t} c_{r_{i}, 1,0} \in \mathbb{N}$.

3. For any prime factor $p$ of $q$ do

3.1. Set $F=\left[\mathbf{c}_{r_{1}, l_{1}}, \mathbf{c}_{r_{2}, l_{2}}, \ldots, \mathbf{c}_{r_{t}, l_{t}}\right] \in \mathbb{Z}_{p}[x]^{n \times t}$.

3.2. Compute a basis $G \subset \mathbb{Z}_{p}[x]^{s}$ of the $\mathbb{Z}_{p}[x]$-module $\operatorname{ker}(F)$ with method in [2, page 74].

3.3. If $G \neq \emptyset$, for each $\mathbf{g}=\left[g_{1}, \ldots, g_{t}\right]^{\tau} \in G, \sum_{i=1}^{t} g_{i} \mathbf{c}_{r_{i}, l_{i}}=p \mathbf{h}$ in $\mathbb{Z}[x]^{n}$.

Return the set of $(\mathbf{h}, p, \mathbf{e})$ where $\mathbf{e} \in \mathbb{Z}[x]^{s}$ and the place of $\mathbf{e}$ corresponding to $\mathbf{c}_{r_{i}, l_{i}}$ is $g_{i}$ and other places are zero.

3.4. Compute the Hermite normal form $\mathcal{B}=\left\{\mathbf{b}_{1}, \ldots, \mathbf{b}_{t}\right\}$ of $\left\{\mathbf{c}_{r_{1}, l_{1}}, \ldots, \mathbf{c}_{r_{t}, l_{t}}\right\}$ in $\mathbb{Z}_{p}[x]^{n}$.

3.5. Let $\mathcal{C}_{-}=\left\{\mathbf{f}_{1}, \ldots, \mathbf{f}_{l}\right\}$ be given in (6) and $\widetilde{\mathbf{f}}_{i}=\operatorname{grem}\left(\mathbf{f}_{i}, \mathcal{B}\right)=\mathbf{f}_{i}+\sum_{k=1}^{t} a_{i, k} \mathbf{b}_{k}$,

in $\mathbb{Z}_{p}[x]^{n}$, where $a_{i, k} \in \mathbb{Z}_{p}[x]$.

3.6. If $\widetilde{\mathbf{f}}_{i}=0$ for some $i$, then $\mathbf{f}_{i}+\sum_{k=1}^{t} a_{i, k} \mathbf{b}_{k}=p_{i} \mathbf{h}_{i}$ in $\mathbb{Z}[x]^{n}$.

Return the set of $\left(\mathbf{h}_{i}, p_{i}, \mathbf{e}_{i}\right)$ where $\mathbf{e}_{i}$ is a vector in $\mathbb{Z}[x]^{s}$ such that

$\left(\mathbf{c}_{1}, \ldots, \mathbf{c}_{s}\right) \mathbf{e}_{i}=\mathbf{f}_{i}+\sum_{k=1}^{t} a_{i, k} \mathbf{b}_{k}=p_{i} \mathbf{h}_{i}$.

3.7. Set $E=\left[\widetilde{\mathbf{f}}_{1}, \ldots, \widetilde{\mathbf{f}}_{l}\right] \in \mathbb{Z}_{p}[x]^{n \times l}$.

3.8. Compute a basis $D$ of $\left\{X \in \mathbb{Z}_{p}^{l} \mid E X=0\right\}$ as a vector space over $\mathbb{Z}_{p}$.

3.9. If $D \neq \emptyset$, for each $\mathbf{b}=\left[b_{1}, \ldots, b_{l}\right]^{\tau} \in D, \sum_{i=1}^{l} b_{i} \widetilde{\mathbf{f}}_{i}=p \mathbf{h}$ in $\mathbb{Z}[x]^{n}$.

Return the set of $(\mathbf{h}, p, \mathbf{e})$ where $\mathbf{e}_{i}$ is a vector in $\mathbb{Z}[x]^{s}$ such that

$\left(\mathbf{c}_{1}, \ldots, \mathbf{c}_{s}\right) \mathbf{e}=\sum_{i=1}^{l} b_{i} \widetilde{\mathbf{f}}_{i}=p \mathbf{h}$.

4. Return $\emptyset$.

Remark 7.3 In steps 3.6 and 3.9, we need to compute $\mathbf{e}_{i}$. Since $\mathcal{B}=\left\{\mathbf{b}_{1}, \ldots, \mathbf{b}_{t}\right\}$ is the Hermite normal form of $\mathbf{c}=\left\{\mathbf{c}_{r_{1}, l_{1}}, \ldots, \mathbf{c}_{r_{t}, l_{t}}\right\}$ in $\mathbb{Z}_{p}[x]^{n}$, there exists an invertible matrix $M_{t \times t}$ such that $\left[\mathbf{b}_{1}, \ldots, \mathbf{b}_{t}\right]=\left[\mathbf{c}_{r_{1}, l_{1}}, \ldots, \mathbf{c}_{r_{t}, l_{t}}\right] M$. In Step 3.6, $\mathbf{e}_{i}$ can be obtained from the relation $\mathbf{f}_{i}+\sum_{k=1}^{t} a_{i, k} \mathbf{b}_{k}=p_{i} \mathbf{h}_{i}$ and the relation $\left[\mathbf{b}_{1}, \ldots, \mathbf{b}_{t}\right]=\left[\mathbf{c}_{r_{1}, l_{1}}, \ldots, \mathbf{c}_{r_{t}, l_{t}}\right] M$. Step 3.9 can be treated similarly.

Remark 7.4 In step 3.8, we need to compute a basis for the vector space $\left\{X \in \mathbb{Z}_{p}^{l} \mid E X=0\right\}$ over $\mathbb{Z}_{p}$. We will show how to do this. A matrix $F \in \mathbb{Z}_{p}[x]^{m \times s}$ is said to be in standard form if $F$ has the structure in (4) and $\operatorname{deg}\left(c_{r_{i}, k_{1}}, x\right)<\operatorname{deg}\left(c_{r_{i}, k_{2}}, x\right)$ for $i=1, \ldots, t$ and $k_{1}<k_{2}$.

The matrix $E \in \mathbb{Z}_{p}[x]^{n \times l}$ can be transformed into standard form using the following operations: (1) exchange two columns and (2) add the multiplication of a column by an element from $\mathbb{Z}_{p}$ to another column. Equivalently, there exists an inversive matrix $U \in \mathbb{Z}_{p}^{l \times l}$ such that $E \cdot U=S$ is in standard form. Suppose that the first $k$ columns of $S$ are zero vectors. Then the first $k$ columns of $U$ constitute a basis for $\operatorname{ker}(E)$. This can be proved similarly to that of the algorithm to compute a basis for the kernel of a matrix over a PID [2, page 74]. 
We now give the algorithm to compute the $\mathbb{Z}$-saturation.

Algorithm $4-\operatorname{SATZ}\left(\mathrm{f}_{0}, \ldots, \mathrm{f}_{s}\right)$

Input: $\quad$ A set of vectors $\mathbb{f}=\left\{\mathbf{f}_{0}, \ldots, \mathbf{f}_{s}\right\} \subset \mathbb{Z}[x]^{n}$.

Output: A generalized Hermite normal form $\mathrm{g}$ such that $(\mathrm{g})=\operatorname{sat}_{\mathbb{Z}}(\mathbb{f})$.

1. Compute a generalized Hermite normal form $\mathfrak{g}$ of $\mathbb{f}$.

2. Set $S=\mathbf{Z F A C T O R}(\mathrm{g})$.

3. If $S=\emptyset$, return $\mathrm{g}$; otherwise set $\mathbb{f}=\mathrm{g} \cup\{\mathbf{h} \mid(\mathbf{h}, k, \mathbf{f}) \in H\}$ and goto step 1 .

Example 7.5 Let $\mathcal{C}$ be the following generalized Hermite normal form:

$$
\mathcal{C}=\left[\begin{array}{lll}
x^{2}+2 x-2 & x+2 & 1 \\
0 & 4 & 2 x
\end{array}\right]
$$

Then, $t=2, r_{1}=1, l_{1}=1, r_{2}=2, l_{2}=2, q=4, \mathbf{c}_{1,1}=\left[x^{2}+2 x-2,0\right]^{\tau}, \mathbf{c}_{2,1}=[x+2,4]^{\tau}$, $\mathbf{c}_{2,2}=[1,2 x]^{\tau}$.

Apply algorithm ZFACTOR to $\mathcal{C}$. We have $p=2$. In steps 3.1 and 3.2, $F=\left[\begin{array}{cc}x^{2} & 1 \\ 0 & 0\end{array}\right]$ and $\operatorname{ker}(F)$ is generated by $G=\left\{\left[-1, x^{2}\right]^{\tau}\right\}$. In step 3.3, $x^{2} \mathbf{c}_{22}-\mathbf{c}_{11}=2\left(1-x, x^{3}\right)^{\tau}$ and return $\left(1-x, x^{3}\right)^{\tau}$.

In Algorithm SATZ, $\left(1-x, x^{3}\right)^{\tau}$ is added into $\mathcal{C}$ and

$$
\mathcal{C}_{1}=\left[\begin{array}{llll}
x^{2}+2 x-2 & x+2 & 1 & 1-x \\
0 & 4 & 2 x & x^{3}
\end{array}\right],
$$

which is also a generalized Hermite normal form.

Applying Algorithm ZFACTOR to $\mathcal{C}_{1}$. We have $p=2$ and $t=2$. In steps 3.1-3.3, $G=\emptyset$. In step $3.4, \mathcal{B}=\left[\begin{array}{ll}x^{2} & 1-x \\ 0 & x^{3}\end{array}\right]$. In step 3.5, $\mathcal{C}_{-}=\left[\begin{array}{lll}x+2 & 1 & x \\ 4 & 2 x & 2 x^{2}\end{array}\right]$ and $\widetilde{\mathbf{f}}_{i} \neq 0$ for all $i$. In step 3.7, $E=\left[\begin{array}{lll}x & 1 & x \\ 0 & 0 & 0\end{array}\right]$. In Step 3.8, $D=\{\mathbf{b}\}$, where $\mathbf{b}=[1,0,-1]^{\tau}$. In Step 3.9, $(x+2,4)^{\tau}-\left(x, 2 x^{2}\right)^{\tau}=2\left(x+1, x^{2}+2\right)^{\tau}$. Add $\left(x+1, x^{2}+2\right)^{\tau}$ is added into $\mathcal{C}_{1}$ and compute the generalized Hermite normal form, we have

$$
\mathcal{C}_{2}=\left[\begin{array}{llll}
x^{2}+2 x-2 & x+2 & 1 & x+1 \\
0 & 4 & 2 x & x^{2}+2
\end{array}\right] .
$$

Apply Algorithm ZFACTOR again, it is shown that $\mathcal{C}_{2}$ is $\mathbb{Z}$-saturated.

We will prove the correctness of the algorithm. We denote by $\operatorname{sat}_{p}(L)$ the set $\{\mathbf{f} \in$ $\left.\mathbb{Z}[x]^{n} \mid p \mathbf{f} \in L\right\}$ where $p \in \mathbb{Z}$ a prime number. An infinite set $S$ is said to be linear independent over a ring $R$ if any finite set of $S$ is linear independent over $R$, that is $\sum_{i=1}^{k} a_{i} \mathbf{g}_{i}=0$ for $a_{i} \in R$ and $\mathbf{g}_{i} \in S$ implies $a_{i}=0, i=1, \ldots, k$.

Lemma 7.6 Let $\mathcal{C}=\left\{\mathbf{c}_{1}, \ldots, \mathbf{c}_{s}\right\}$ be a generalized Hermite normal form and $L=(\mathcal{C})$. Then $\operatorname{sat}_{p}(L)=L$ if and only if $\mathcal{C}_{\infty}$ is linear independent over $\mathbb{Z}_{p}$, where $\mathcal{C}_{\infty}$ is defined in (6). 
Proof: " $\Rightarrow$ " Assume the contrary, that is, $\mathcal{C}_{\infty}=\left\{\mathbf{h}_{1}, \mathbf{h}_{2}, \ldots\right\}$ defined in (6) is linear dependent over $\mathbb{Z}_{p}$. Then there exist $a_{i} \in \mathbb{Z}_{p}$ not all zero, such that $\sum_{i=1}^{r} a_{i} \mathbf{h}_{i}=0$ in $\mathbb{Z}_{p}[x]^{n}$ and hence $\sum_{i=1}^{r} a_{i} \mathbf{h}_{i}=p \mathbf{g}$ in $\mathbb{Z}[x]^{n}$. By Lemma 3.14, $\mathbf{h}_{i}$ are linear independent over $\mathbb{Z}_{p}$ and hence $\mathbf{g} \neq \mathbf{0}$. Since $\operatorname{sat}_{p}(L)=L$, we have $\mathbf{g} \in L$. By Lemma 3.15, there exist $b_{i} \in \mathbb{Z}$ such that $\mathbf{g}=\sum_{i=1}^{r} b_{i} \mathbf{h}_{i}$. Hence $\sum_{i=1}^{r}\left(a_{i}-p b_{i}\right) \mathbf{h}_{i}=0$ in $\mathbb{Z}[x]^{n}$. By Lemma 3.14, $a_{i}=p b_{i}$ and hence $a_{i}=0$ in $\mathbb{Z}_{p}[x]$, a contradiction.

" $\Leftarrow "$ Assume the contrary, that is, there exists a $\mathbf{g} \in \mathbb{Z}[x]^{n}$, such that $\mathbf{g} \notin L$ and $p \mathbf{g} \in L$. By Lemma 3.15, $p \mathbf{g}=\sum_{i=1}^{r} a_{i} \mathbf{h}_{i}$, where $a_{i} \in \mathbb{Z} . p$ cannot be a factor of all $a_{i}$. Otherwise, $\mathbf{g}=\sum_{i=1}^{r} \frac{a_{i}}{p} \mathbf{h}_{i} \in L$. Then some of $a_{i}$ is not zero in $\mathbb{Z}_{p}$, which means $\sum_{i=1}^{r} a_{i} \mathbf{h}_{i}=0$ is nontrivial linear relation among $\mathcal{C}_{i}$ over $\mathbb{Z}_{p}$, a contradiction.

From the " $\Rightarrow$ " part of the above proof, we have

Corollary 7.7 Let $\sum_{i=1}^{r} a_{i} \mathbf{h}_{i}=0$ be a nontrivial linear relation among $\mathbf{h}_{i}$ in $\mathbb{Z}_{p}[x]^{n}$, where $a_{i} \in \mathbb{Z}_{p}$. Then, in $\mathbb{Z}[x]^{n}, \sum_{i=1}^{r} a_{i} \mathbf{h}_{i}=p \mathbf{h}$ and $\mathbf{h} \notin(\mathcal{C})$.

Lemma 7.8 Let $\mathcal{C}=\left\{\mathbf{c}_{1}, \ldots, \mathbf{c}_{s}\right\}$ be a generalized Hermite normal form and $L=(\mathcal{C})$. Then $\operatorname{sat}_{p}(L)=L$ if and only if $\mathcal{C}_{\infty}$ is linear independent over $\mathbb{Z}_{p}$ for the prime factors of $q$ defined in step 2 of Algorithm ZFACTOR.

Proof: By Definition 3.8, the leading monomial of $x^{k} \mathbf{c}_{r_{i}, j} \in \mathcal{C}_{\infty}$ is of the form $c_{r_{i}, j, 0} x^{k+d_{r_{i}, j}} \boldsymbol{\epsilon}_{r_{i}}$ and $c_{r_{i}, l_{i}, 0}|\ldots| c_{r_{i}, 2,0} \mid c_{r_{i}, 1,0}$. If $p$ is coprime with $\prod_{i=1}^{t} c_{r_{i}, 1,0}$, then $c_{r_{i}, j, 0} \neq 0 \bmod p$ for $1 \leq j \leq l_{i}$. Therefore, the leading monomials of the elements of $\mathcal{C}_{\infty}$ are linear independent over $\mathbb{Z}_{p}$, and hence $\mathcal{C}_{\infty}$ is linear independent over $\mathbb{Z}_{p}$. Therefore, it suffices to consider prime factors of $\prod_{i=1}^{t} c_{r_{i}, 1,0}$.

To check whether $\mathcal{C}_{\infty}$ is linear independent over $\mathbb{Z}_{p}$, we first consider a subset of $\mathcal{C}_{\infty}$ is linear independent in the following lemma.

Lemma 7.9 Let $\mathcal{C}$ be the generalized Hermite normal form given in (4). Then $\mathcal{C}^{+}$defined in (6) is linear independent over $\mathbb{Z}_{p}$ if and only if $\left\{\mathbf{c}_{r_{1}, l_{1}}, \mathbf{c}_{r_{2}, l_{2}}, \ldots, \mathbf{c}_{r_{t}, l_{t}}\right\}$ are linear independent over $\mathbb{Z}_{p}[x]$.

Proof: This is obvious since $\sum_{i} \sum_{j} a_{i, j} x^{j} \mathbf{c}_{r_{i}, l_{i}}=\sum_{i} p_{i} \mathbf{c}_{r_{i}, l_{i}}$, where $a_{i, j} \in \mathbb{Z}$ and $p_{i}=$ $\sum_{j} a_{i, j} x^{j}$

Lemma 7.10 Let $\mathcal{B}$ be an Hermite normal form in $\mathbb{Z}_{p}[x]^{n}$ and $\mathrm{g}=\left\{\mathbf{g}_{1}, \ldots, \mathbf{g}_{r}\right\} \subset \mathbb{Z}_{p}[x]^{n}$. Then $\mathrm{g} \cup \mathcal{B}_{\infty}$ is linear dependent over $\mathbb{Z}_{p}$ if and only if

- either $\widetilde{\mathbf{g}}_{i}=\operatorname{grem}\left(\mathbf{g}_{i}, \mathcal{B}\right)=0$ in $\mathbb{Z}_{p}[x]^{n}$ for some $i$, or

- the residue set $\left\{\operatorname{grem}\left(\mathbf{g}_{i}, \mathcal{B}\right) \mid i=1, \ldots, r\right\}$ are linear dependent over $\mathbb{Z}_{p}$.

Proof: We may assume that $\operatorname{grem}\left(\mathbf{g}_{i}, \mathcal{B}\right)=0$ does not happen, since it gives a nontrivial linear relation of $\mathrm{g} \cup \mathcal{B}_{\infty}$. By Lemma 3.15, $\widetilde{\mathrm{g}}_{i}=\mathrm{g}_{i} \bmod \mathcal{B}_{\infty} . \mathrm{g} \cup \mathcal{B}_{\infty}$ is linear dependent over $\mathbb{Z}_{p}$ if and only if there exist $a_{i} \in \mathbb{Z}_{p}$ not all zero such that $\sum_{i} a_{i} \mathbf{g}_{i}=0 \bmod \mathcal{B}_{\infty}$ over $\mathbb{Z}_{p}$, 
which is valid if and only if $\sum_{i} a_{i} \widetilde{\mathbf{g}_{i}}=0 \bmod \mathcal{B}_{\infty}$. Since $\widetilde{\mathbf{g}}_{i}$ are G-reduced with respect to $\mathcal{B}$, $\sum_{i} a_{i} \widetilde{\mathbf{g}}_{i}=0 \bmod \mathcal{B}_{\infty}$ if and only if $\sum_{i} a_{i} \widetilde{\mathbf{g}}_{i}=0$, that is $\widetilde{\mathbf{g}}_{i}$ are linear dependent over $\mathbb{Z}_{p}$.

Theorem 7.11 Algorithm $\mathbf{S A T Z}\left(\mathbf{f}_{0}, \ldots, \mathbf{f}_{s}\right)$ is correct.

Proof: Since $\mathbb{Z}[x]^{n}$ is Notherian, it suffices to show that Algorithm ZFACTOR is correct. Let $C=\left\{\mathbf{c}_{1}, \ldots, \mathbf{c}_{s}\right\}$. By Lemma 7.6, to check whether $\operatorname{sat}_{\mathbb{Z}}\left(\mathbf{c}_{1}, \ldots, \mathbf{c}_{s}\right)$ is $\mathbb{Z}$-saturated, we need only to check for any $p$ prime, $\mathcal{C}_{\infty}$ is linear independent on $\mathbb{Z}_{p}$.

By Lemma 7.8, we need only to consider prime factors of $\prod_{i=1}^{t} c_{r_{i}, 1,0}$ in step 3 of the algorithm.

In steps 3.1 and 3.2 , we check whether $\mathcal{C}^{+}$in (6) is linear independent over $\mathbb{Z}_{p}$. By Lemma 7.9. we need only to consider whether $\mathcal{C}_{1}=\left\{\mathbf{c}_{r_{1}, l_{1}}, \mathbf{c}_{r_{2}, l_{2}}, \ldots, \mathbf{c}_{r_{t}, l_{t}}\right\}$ is linear independent over $\mathbb{Z}_{p}[x]$. It is clear that $\mathcal{C}_{1}$ is linear independent over $\mathbb{Z}_{p}[x]$ if and only if $G=\emptyset$, where $\mathbb{G}$ is given in step 3.2.

In step 3.3, we handle the case that $\mathcal{C}_{1}$ is linear dependent over $\mathbb{Z}_{p}$. If $G \neq \emptyset$ for any $\mathbf{g}=\left[g_{1}, \ldots, g_{t}\right]^{\tau} \in G, \sum_{i=1}^{t} g_{i} \mathbf{c}_{r_{i}, l_{i}}=0$ in $\mathbb{Z}_{p}[x]$. Hence $\sum_{i=1}^{t} g_{i} \mathbf{c}_{r_{i}, l_{i}}=p \mathbf{h}$ where $\mathbf{h} \in \mathbb{Z}[x]^{n}$. By Corollary [7.7, $\mathbf{h} \notin L$. The correctness of Algorithm ZFACTOR is proved in this case.

In steps $3.4-3.10$, we handle the case where $\mathcal{C}^{+}$is linear independent over $\mathbb{Z}_{p}$. In step 3.4 , we compute the Hermite normal form of $\mathcal{C}_{1}$ in $\mathbb{Z}_{p}[x]^{n}$, which is possible because $\mathbb{Z}_{p}[x]^{n}$ is a PID [2]. Furthermore, we have [2]

$$
\left[\mathbf{c}_{r_{1}, l_{1}}, \ldots, \mathbf{c}_{r_{t}, l_{t}}\right] N=\left[\mathbf{b}_{1}, \ldots, \mathbf{b}_{t}\right]
$$

where $\left\{\mathbf{b}_{1}, \ldots, \mathbf{b}_{t}\right\}$ is an Hermite normal form and $N$ is an inversive matrix in $\mathbb{Z}_{p}[x]^{t \times t}$. Then $\mathcal{C}_{\infty}=\mathcal{C}_{-} \cup \mathcal{C}^{+}$is linear independent over $\mathbb{Z}_{p}$ if and only if

$$
\widetilde{\mathcal{C}}=\mathcal{C}_{-} \cup \mathcal{B}_{\infty}=\mathcal{C}_{-} \cup \cup_{j=0}^{\infty}\left\{x^{j} \mathbf{b}_{1}, \ldots, x^{j} \mathbf{b}_{t}\right\} \text { is linear independent over } \mathbb{Z}_{p} .
$$

By Lemma 7.10, property (43) is valid if and only if $\operatorname{grem}(\mathbf{c}, \mathcal{B}) \neq 0$ for all $\mathbf{c} \in \mathcal{C}_{-}$and the residue set $\widetilde{\mathcal{C}}_{-}$is linear independent over $\mathbb{Z}_{p}$, which are considered in step 3.7 and steps 3.83.10 , respectively. Then we either prove $L$ is $\mathbb{Z}$-saturated or find a nontrivial linear relation for elements in $\mathcal{C}_{\infty}$ over $\mathbb{Z}_{p}$. By Corollary 7.7 , such a relation leads to an $\mathbf{h} \in \operatorname{sat}_{\mathbb{Z}}(L) \backslash L$. The correctness of the algorithm is proved.

As a direct consequence of Lemma 4.42 and Algorithm ZFACTOR, we have the algorithm to compute the M-saturation.

Algorithm 5-SATM $\left(f_{0}, \ldots, f_{s}\right)$

Input: $\quad A$ set of vectors $\mathbb{f}=\left\{\mathbf{f}_{0}, \ldots, \mathbf{f}_{s}\right\} \subset \mathbb{Z}[x]^{n}$.

Output: A generalized Hermite normal form $\mathrm{g}$ such that $\operatorname{sat}_{M}(\mathbb{f})=(\mathrm{g})$.

1. Using Algorithm ZFACTOR, we can compute $m_{i} \in \mathbb{N}$ and $\mathbf{g}_{i} \in \mathbb{Z}[x]^{n}, i=1, \ldots, s$ such that $\operatorname{sat}_{\mathbb{Z}}(\mathbb{H})=\left(\mathbf{g}_{1}, \ldots, \mathbf{g}_{s}\right)$ and $m_{i} \mathbf{g}_{i} \in(\mathbb{f})$.

2. Let $S=\emptyset$ and for $i=1, \ldots, s$, if $m_{i} \neq 1$ then $S=S \cup\left\{\left(x-o_{m_{i}}\right) \mathbf{g}_{i}\right\}$.

3. Compute the generalized Hermite normal form g of $\mathbb{f} \cup S$ and return g. 
Notices that if $m_{i}=1$ then $o_{m_{i}}=0$ and $\mathbf{g}_{i} \in(\mathbb{f})$. The numbers $m_{i}$ need not to be unique for the following reasons. Suppose $m_{i}=n_{i} k$ and $n_{i} \mathbf{g}_{i} \in(\mathbb{f})$. Then by Corollary 4.37, $o_{m_{i}}=o_{n_{i}}+c n_{i}$ and hence $\left(x-o_{n_{i}}\right) \mathbf{g}_{i}=\left(x-o_{m_{i}}\right) \mathbf{g}_{i}+c n_{i} \mathbf{g}_{i} \in(\mathbb{f})$. That is, we can replace $m_{i}$ by its factor $n_{i}$.

Lemma 7.12 Algorithm SATM is correct.

Proof: Let $L_{1}=(\mathbb{f})$ and $L_{2}=\left(\mathbb{f},\left(x-o_{m_{1}}\right) \mathbf{g}_{1}, \ldots,\left(x-o_{m_{s}}\right) \mathbf{g}_{s}\right)$. We claim that $\operatorname{sat}_{\mathbb{Z}}\left(L_{1}\right)=$ $\operatorname{sat}_{\mathbb{Z}}\left(L_{2}\right)$. Since $L_{1} \subset L_{2}, \operatorname{sat}_{\mathbb{Z}}\left(L_{1}\right) \subset \operatorname{sat}_{\mathbb{Z}}\left(L_{2}\right)$. Since $\operatorname{sat}_{\mathbb{Z}}\left(L_{1}\right)=\left(\mathbf{g}_{1}, \ldots, \mathbf{g}_{s}\right)$, we have $L_{2} \subset$ $\operatorname{sat}_{\mathbb{Z}}\left(L_{1}\right)$ and hence $\operatorname{sat}_{\mathbb{Z}}\left(L_{2}\right) \subset \operatorname{sat}_{\mathbb{Z}}\left(L_{1}\right)$. The claim is proved. Then $\operatorname{sat}_{\mathbb{Z}}\left(L_{2}\right)=\left(\mathbf{g}_{1}, \ldots, \mathbf{g}_{s}\right)$ and $m_{i} \mathbf{g}_{i} \in L_{1} \subset L_{2}$. Since $\left(x-o_{m_{i}}\right) \mathbf{g}_{i} \in L_{2}, i=1, \ldots, s, L_{2}$ is M-saturated by Lemma 4.42 ,

\section{$7.3 \mathbb{Z}[x]$-saturation of $\mathbb{Z}[x]$-lattice}

The following algorithm checks whether a generalized Hermite normal form $\mathcal{C}$ is $\mathbb{Z}[x]$-saturated or toric, and if not, it will return a set of elements in $\operatorname{sat}_{\mathbb{Z}[x]}(\mathcal{C}) \backslash(\mathcal{C})$.

$\overline{\text { Algorithm } 6 \text { - ZXFACTOR }\left(\mathbf{c}_{1}, \ldots, \mathrm{c}_{s}\right)}$

Input: A generalized Hermite normal form $\mathcal{C}=\left\{\mathbf{c}_{1}, \ldots, \mathbf{c}_{s}\right\} \subset \mathbb{Z}[x]^{n}$ given in (4).

Output: $\emptyset$, if $L=(\mathcal{C})$ is $\mathbb{Z}[x]$-saturated; otherwise, a finite set $\left\{\mathbf{h}_{1}, \ldots, \mathbf{h}_{r}\right\} \subset \mathbb{Z}[x]^{n}$ such that $\mathbf{h}_{i} \notin L$ and $\mathbf{h}_{i} \in \operatorname{sat}_{\mathbb{Z}[x]}(L), i=1, \ldots, r$.

1. Let $S=\mathbf{Z F A C T O R}(\mathcal{C})$. If $S \neq \emptyset$ return $S$.

2. For any prime factor $p(x)$ of $\prod_{i=1}^{t} c_{r_{i}, 1} \in \mathbb{Z}[x] \backslash \mathbb{Z}$.

2.1. Set $M=\left[\mathbf{c}_{r_{1}, 1}, \ldots, \mathbf{c}_{r_{t}, 1}\right] \in \mathbb{Z}[x]^{n \times t}$, where $\mathbf{c}_{r_{i}, 1}$ can be found in (4).

2.2. Compute a finite basis $B=\left\{\mathbf{b}_{1}, \ldots, \mathbf{b}_{l}\right\}$ of $\operatorname{ker}(M)=\left\{X \in \mathbb{Q}[x]^{t} \mid M X=0\right\}$

as a vector space in $(\mathbb{Q}[x] /(p(x)))^{t}$.

2.3. If $B \neq \emptyset$,

2.3.1. For each $\mathbf{b}_{i}$, let $M \mathbf{b}_{i}=p(x) \frac{\mathbf{g}_{i}}{m_{i}}$, where $\mathbf{g}_{i} \in \mathbb{Z}[x]^{n}$ and $m_{i} \in \mathbb{Z}$.

2.3.2. Return $\left\{\mathbf{g}_{1}, \ldots, \mathbf{g}_{l}\right\}$

3. Return $\emptyset$.

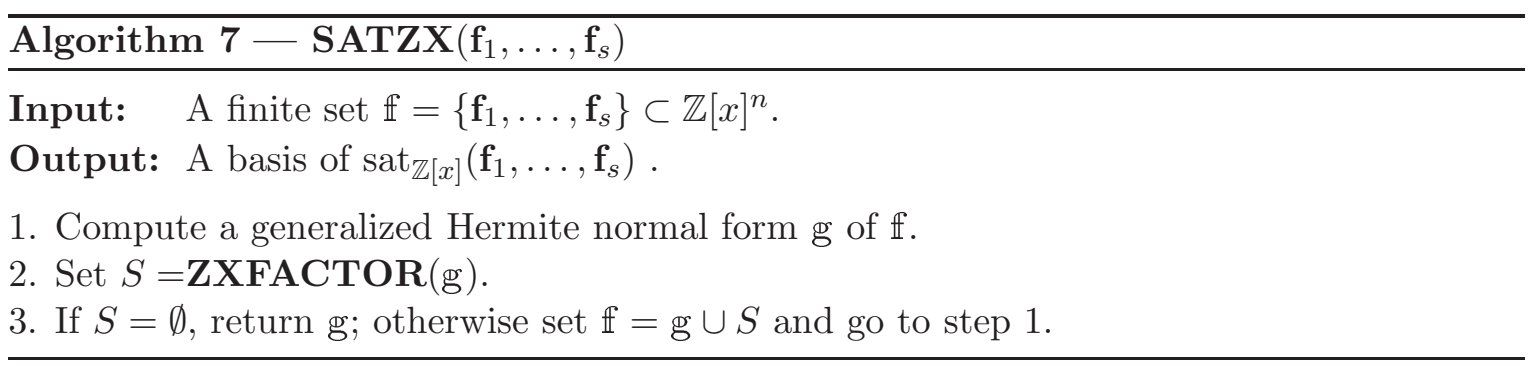


Example 7.13 Let

$$
\mathcal{C}=\left[\begin{array}{ll}
x & x^{2}+1 \\
2 x^{2}+1 & 0 \\
0 & 4 x^{2}+2
\end{array}\right] .
$$

Apply Algorithm ZXFACTOR to $\mathcal{C}$. In step, $1, S=\emptyset$ and $\mathcal{C}$ is $\mathbb{Z}$-saturated. In step 2, the only irreducible factor of $\prod_{i=1}^{t} c_{r_{i}, 1} \in \mathbb{Z}[x]$ is $p(x)=2 x^{2}+1$. In step 2.1, $M=\mathcal{C}$ and in step 2.2, $B=\left\{[-1,2 x]^{\tau}\right\}$. In step 2.3.1, $M \cdot[-1,2 x]^{\tau}=2 x \mathbf{c}_{2,1}-\mathbf{c}_{1,1}=p(x)[x,-1,4 x]^{\tau}=$ $0 \bmod p(x)$ and $\left\{[x,-1,4 x]^{\tau}\right\}$ is returned.

In Algorithm SATZX, $\mathbf{h}=[x,-1,4 x]^{\tau}$ is added into $\mathcal{C}$ and the generalized Hermite normal form of $\mathcal{C} \cup\{\mathbf{h}\}$ is

$$
\mathcal{C}_{1}=\left[\begin{array}{ll}
x & 1 \\
2 x^{2}+1 & x \\
0 & 2
\end{array}\right] .
$$

Apply Algorithm ZXFACTOR to $\mathcal{C}_{1}$, one can check that $\mathcal{C}_{1}$ is $\mathbb{Z}[x]$-saturated.

In the rest of this section, we will prove the correctness of the algorithm. Denote $L_{\mathbb{Q}}$ to be the $\mathbb{Q}[x]$-module generated by $L$ in $\mathbb{Q}[x]^{n}$. Similar to the definition of $\operatorname{sat}_{\mathbb{Z}[x]}(L)$, we can define $\operatorname{sat}_{\mathbb{Q}[x]}\left(L_{\mathbb{Q}}\right) . \quad L_{\mathbb{Q}}$ is called $\mathbb{Q}[x]$-saturated if $\operatorname{sat}_{\mathbb{Q}[x]}\left(L_{\mathbb{Q}}\right)=L_{\mathbb{Q}}$. The following lemma gives a criterion for whether $L$ is $\mathbb{Z}[x]$-saturated.

Lemma 7.14 $A \mathbb{Z}[x]$-lattice $L$ is $\mathbb{Z}[x]$-saturated if and only if $\operatorname{sat}_{\mathbb{Z}}(L)=L$ and $\operatorname{sat}_{\mathbb{Q}[x]}\left(L_{\mathbb{Q}}\right)=$ $L_{\mathbb{Q}}$.

Proof: " $\Rightarrow$ " If $L=\left(\mathbf{f}_{1}, \ldots, \mathbf{f}_{s}\right)$ is $\mathbb{Z}[x]$-saturated, then $\operatorname{sat}_{\mathbb{Z}}(L)=L$. If $\operatorname{sat}_{\mathbb{Q}[x]}\left(L_{\mathbb{Q}}\right) \neq L_{\mathbb{Q}}$, then there exists an $h(x) \in \mathbb{Q}[x]$ and a $\mathbf{g} \in \mathbb{Q}[x]^{n}$, such that $h(x) \mathbf{g} \in L_{\mathbb{Q}}$ but $\mathbf{g} \notin L_{\mathbb{Q}}$. From $h(x) \mathbf{g} \in L_{\mathbb{Q}}$, we have $h(x) \mathbf{g}=\sum_{i=1}^{s} q_{i}(x) \mathbf{f}_{i}$ where $q_{i}(x) \in \mathbb{Q}[x]$. By clearing the denominators of the above equation, there exist $m_{1}, m_{2} \in \mathbb{Z}$ such that $m_{1} h(x) \in \mathbb{Z}[x], m_{2} \mathbf{g} \in \mathbb{Z}[x]^{n}$, and $m_{1} h(x) \cdot m_{2} \mathbf{g} \in L$. Since $L$ is $\mathbb{Z}[x]$-saturated, $m_{2} \mathbf{g} \in L$, which contradicts to $\mathbf{g} \notin L_{\mathbb{Q}}$.

" $\Leftarrow "$ For any $h(x) \in \mathbb{Z}[x]$ and $\mathbf{g} \in \mathbb{Z}[x]^{n}$, if $h(x) \mathbf{g} \in L$, we have $h(x) \mathbf{g} \in L_{\mathbb{Q}}$, and hence $\mathbf{g} \in L_{\mathbb{Q}}$ since $\operatorname{sat}_{\mathbb{Q}[x]}\left(L_{\mathbb{Q}}\right)=L_{\mathbb{Q}}$. From $\mathbf{g} \in L_{\mathbb{Q}}$, there exists an $m \in \mathbb{Z}$ such that $m \mathbf{g} \in L$ which implies $\mathbf{g} \in L$ since $L$ is $\mathbb{Z}$-saturated.

The following lemma shows that a generalized Hermite normal form becomes an Hermite normal form in $\mathbb{Q}[x]^{n}$.

Lemma 7.15 Let $\mathcal{C}$ be the generalized Hermite normal form given in (4). Then $(\mathcal{C})=$ $\left(\mathbf{c}_{r_{1}, 1}, \ldots, \mathbf{c}_{r_{t}, 1}\right)$ as $\mathbb{Q}[x]$-modules in $\mathbb{Q}[x]^{n}$ and $\left[\mathbf{c}_{r_{1}, 1}, \ldots, \mathbf{c}_{r_{t}, 1}\right]$ is an Hermite normal form.

Proof: It is clear that $\left[\mathbf{c}_{r_{1}, 1}, \ldots, \mathbf{c}_{r_{t}, 1}\right]$ is an Hermite normal form. We will prove $(\mathcal{C})=$ $\left(\mathbf{c}_{r_{1}, 1}, \ldots, \mathbf{c}_{r_{t}, 1}\right)$ by induction. By 3) of Definition 3.8, $S\left(\mathbf{c}_{r_{1}, 1}, \mathbf{c}_{r_{1}, 2}\right)=x^{u} \mathbf{c}_{r_{1}, 1}-a \mathbf{c}_{r_{1}, 2}(u \in \mathbb{N}$ and $a \in \mathbb{Z}$ ) can be reduced to zero by $\mathbf{c}_{r_{1}, 1}$, which means $\mathbf{c}_{r_{1}, 2}=q(x) \mathbf{c}_{r_{1}, 1}$ where $q(x) \in \mathbb{Q}[x]$. Hence, $\left(\mathbf{c}_{r_{1}, 1}, \mathbf{c}_{r_{1}, 2}\right)=\left(\mathbf{c}_{r_{1}, 1}\right)$ as $\mathbb{Q}[x]$-modules. Suppose for $k<l_{1},\left(\mathbf{c}_{r_{1}, 1}, \ldots, \mathbf{c}_{r_{1}, k}\right)=\left(\mathbf{c}_{r_{1}, 1}\right)$ as $\mathbb{Q}[x]$-modules. We will show that $\left(\mathbf{c}_{r_{1}, 1}, \ldots, \mathbf{c}_{r_{1}, k+1}\right)=\left(\mathbf{c}_{r_{1}, 1}\right)$ as $\mathbb{Q}[x]$-modules. Indeed, by 3) of Definition [3.8, $S\left(\mathbf{c}_{r_{1}, 1}, \mathbf{c}_{r_{1}, k+1}\right)=x^{v} \mathbf{c}_{r_{1}, 1}-b \mathbf{c}_{r_{1}, k+1}(v \in \mathbb{N}$ and $b \in \mathbb{Z})$ can be reduced to zero by $\mathbf{c}_{r_{1}, 1}, \ldots, \mathbf{c}_{r_{1}, k}$ and hence, $\mathbf{c}_{r_{1}, k+1} \in\left(\mathbf{c}_{r_{1}, 1}\right)$ as $\mathbb{Q}[x]$-modules. Then we 
have $\left(\mathbf{c}_{r_{1}, 1}, \ldots, \mathbf{c}_{r_{1}, l_{1}}\right)=\left(\mathbf{c}_{r_{1}, 1}\right)$ as $\mathbb{Q}[x]$-modules. For the rest of the polynomials in $\mathcal{C}$, the proof is similar.

The following lemma gives a criterion for a $\mathbb{Q}[x]$-module to be $\mathbb{Q}[x]$-saturated.

Lemma 7.16 Let $\mathcal{C}$ be the generalized Hermite normal form given in (4) and $L=(\mathcal{C})$. Then $\operatorname{sat}_{\mathbb{Q}[x]}\left(L_{\mathbb{Q}}\right)=L_{\mathbb{Q}}$ if and only if $\mathcal{C}_{1}=\left\{\mathbf{c}_{r_{1}, 1}, \ldots, \mathbf{c}_{r_{t}, 1}\right\}$ is linear independent over $\mathbb{K}_{p(x)}=\mathbb{Q}[x] /(p(x))$ for any irreducible polynomial $p(x) \in \mathbb{Z}[x]$.

Proof: " $\Rightarrow$ " Assume the contrary, that is, $\mathcal{C}_{1}$ are linear dependent over $\mathbb{K}_{p(x)}$ for some $p(x)$. Then there exist $g_{i} \in \mathbb{Q}[x]$ not all zero in $\mathbb{K}_{p(x)}$, such that $\sum_{i=1}^{t} g_{i} \mathbf{c}_{r_{i}, 1}=0$ in $\mathbb{K}_{p(x)}^{n}$ and hence $\sum_{i=1}^{t} g_{i} \mathbf{c}_{r_{i}, 1}=p(x) \mathbf{g}$ in $\mathbb{Q}[x]^{n}$. Since $\mathcal{C}_{1}$ is in triangular form and is clearly linear independent in $\mathbb{Q}[x]^{n}$, we have $\mathbf{g} \neq \mathbf{0}$. Since $\operatorname{sat}_{\mathbb{Q}[x]}\left(L_{\mathbb{Q}}\right)=L_{\mathbb{Q}}$, we have $\mathbf{g} \in L_{\mathbb{Q}}$. Then, there exist $f_{i} \in \mathbb{Q}[x]$ such that $\mathbf{g}=\sum_{i=1}^{t} f_{i} \mathbf{c}_{r_{i}, 1}$. Hence $\sum_{i=1}^{t}\left(g_{i}-p f_{i}\right) \mathbf{c}_{r_{i}, 1}=0$ in $\mathbb{Q}[x]^{n}$. Since $\mathcal{C}_{1}$ is linear independent in $\mathbb{Q}[x]^{n}, g_{i}=p f_{i}$ and hence $g_{i}=0$ in $\mathbb{K}_{p(x)}$, a contradiction.

" $\Leftarrow "$ Assume the contrary, that is, there exists a $\mathbf{g} \in \mathbb{Q}[x]^{n}$, such that $\mathbf{g} \notin L_{\mathbb{Q}}$ and $p(x) \mathbf{g} \in L_{\mathbb{Q}}$ for an irreducible polynomial $p(x) \in \mathbb{Z}[x]$. Then, by Lemma 7.15 we have $p \mathbf{g}=$ $\sum_{i=1}^{t} f_{i} \mathbf{c}_{r_{i}, 1}$, where $f_{i} \in \mathbb{Q}[x] . p$ cannot be a factor of all $f_{i}$. Otherwise, $\mathbf{g}=\sum_{i=1}^{t} \frac{f_{i}}{p} \mathbf{c}_{r_{i}, 1} \in$ $L_{\mathbb{Q}}$. Then some of $f_{i}$ is not zero in $\mathbb{K}_{p(x)}$, which means $\sum_{i=1}^{t} f_{i} \mathbf{c}_{r_{i}, 1}=0$ is a nontrivial linear relation among $\mathcal{C}_{1}$ over $\mathbb{K}_{p(x)}$, a contradiction.

From the " $\Rightarrow$ " part of the above proof, we have

Corollary 7.17 Let $\mathcal{C}$ be the generalized Hermite normal form given in (4) and $\sum_{i=1}^{t} f_{i} \mathbf{c}_{r_{i}, 1}$ $=0$ a nontrivial linear relation among $\mathbf{c}_{r_{i}, 1}$ in $(\mathbb{Q}[x] /(p(x)))^{n}$, where $p(x)$ is an irreducible polynomial in $\mathbb{Z}[x]$ and $f_{i} \in \mathbb{Q}[x]$. Then, in $\mathbb{Q}[x]^{n}, \sum_{i=1}^{r} f_{i} \mathbf{c}_{r_{i}, 1}=p(x) \mathbf{g}$ and $\mathbf{g} \notin(\mathcal{C})$ as a $\mathbb{Q}[x]$-module.

Theorem 7.18 Algorithm $\operatorname{SATZX}\left(\mathbf{f}_{1}, \ldots, \mathbf{f}_{s}\right)$ is correct.

Proof: Since $\mathbb{Z}[x]^{n}$ is Notherian, we need only to show the correctness of Algorithm ZXFACTOR. In step $1, L$ is $\mathbb{Z}$ saturated if and only if $S \neq \emptyset$. In step 2 , we claim that $L$ is $\mathbb{Q}[x]$-saturated if and only if $B=\emptyset$ and if $B \neq \emptyset$ then $\mathbf{g}_{i}$ in step 2.3.1 is not in $L$. In step 3, $L$ is both $\mathbb{Z}$ and $\mathbb{Q}[x]$ saturated. By Lemma $7.14, L$ is $\mathbb{Z}[x]$-saturated and the algorithm is correct. So, it suffices to prove the claim about step 2 .

By Lemma 7.16, to check whether $\operatorname{sat}_{\mathbb{Q}[x]}\left(L_{\mathbb{Q}}\right)=L_{\mathbb{Q}}$, we need only to check whether for any irreducible polynomial $p(x) \in \mathbb{Z}[x], \mathcal{C}_{1}=\left\{\mathbf{c}_{r_{1}, 1}, \ldots, \mathbf{c}_{r_{t}, 1}\right\}$ is linear independent over $\mathbb{K}_{p(x)}=\mathbb{Q}[x] /(p(x))$. If $p(x)$ is not a prime factor of $\prod_{i=1}^{t} c_{r_{i}, 1}$, then the leading monomials of $\mathbf{c}_{r_{i}, 1}, i=1, \ldots, t$ are nonzero. Since $\mathcal{C}_{1}$ is an Hermite normal form, $\mathcal{C}_{1}$ is linear independent over $\mathbb{K}_{p(x)}$. Hence, we need only to consider prime factors of $\prod_{i=1}^{t} c_{r_{i}, 1}$ in step 2 of the algorithm. In step 2.3, it is clear that if $\mathcal{C}_{1}=\emptyset$ then $\mathcal{C}_{1}$ is linear independent over $\mathbb{K}_{p(x)}$. For $\mathbf{b}_{i} \in B$, since $M \mathbf{b}_{i}=0$ over $\mathbb{K}_{p(x)}, M \mathbf{b}_{i}=p(x) \mathbf{h}_{i}$ where $\mathbf{h}_{i} \in \mathbb{Q}[x]^{t}$. Hence $\mathbf{h}_{i}=\frac{\mathbf{g}_{i}}{m_{i}}$ for $\mathbf{g}_{i} \in \mathbb{Z}[x]^{t}$ and $m_{i} \in \mathbb{Z}$. By Corollary [7.17, $\mathbf{g}_{i} \notin L$. Therefore, step 2 returns a set of nontrivial factors of $L$ if $L$ is not $\mathbb{Z}[x]$-saturated. The claim about step 2 is proved. 


\subsection{Algorithms for binomial $\sigma$-ideals}

In this section, we will present several algorithms for Laurent binomial and binomial $\sigma$-ideals, and in particular a decomposition algorithm for binomial $\sigma$-ideals. We first give an algorithm to compute the reflexive closure for a Laurent binomial $\sigma$-ideal.

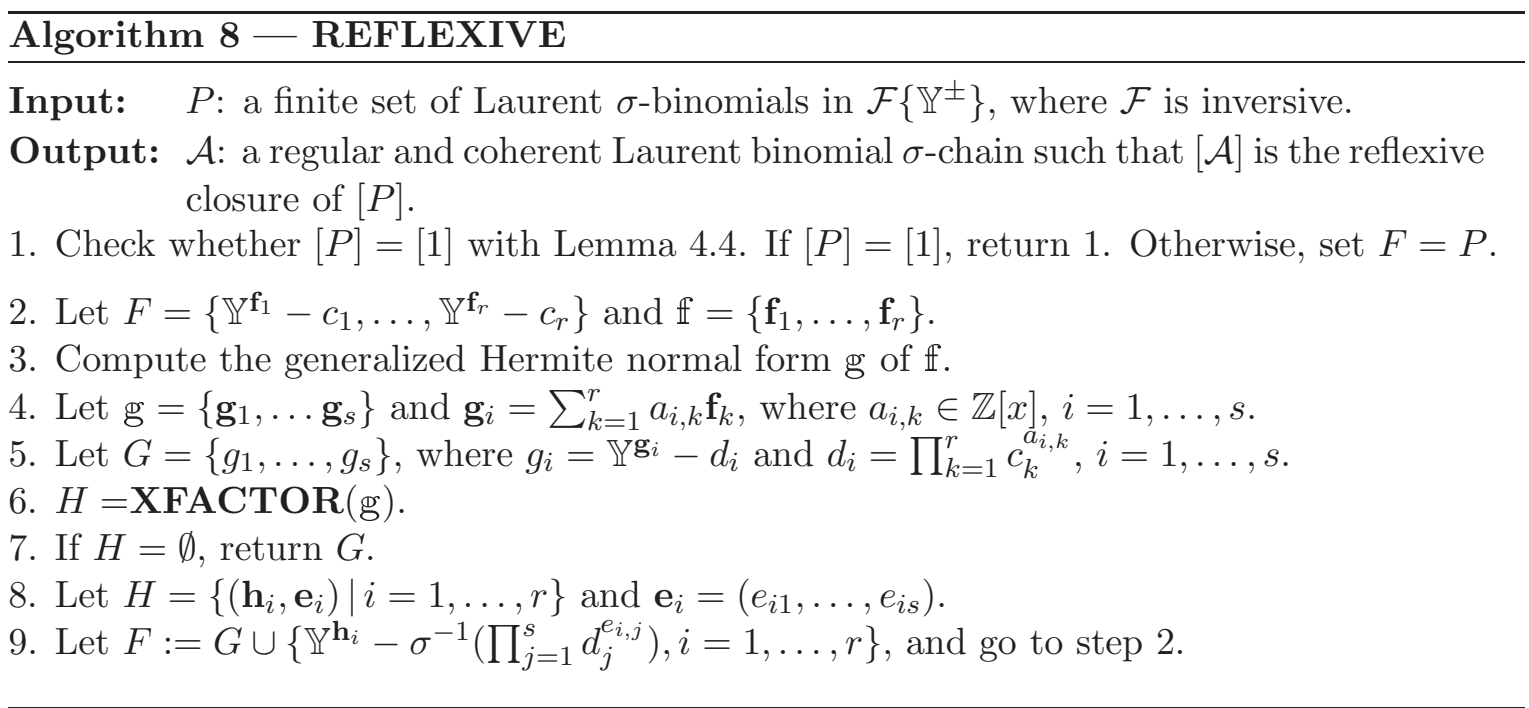

Theorem 7.19 Algorithm REFLEXIVE is correct.

Proof: The algorithm basically follows the proof of Theorem 4.26. In steps 2-5, $(\mathbb{f})$ and $(\mathrm{g})$ are the support lattices of $[F]$ and $[G]$, respectively. By Lemma $4.3,[F]=[G]$. By Lemma 4.9. $G$ is a regular and coherent $\sigma$-chain. In step 7 , if $H=\emptyset$, then $(\mathrm{g})$ is $x$-saturated, and by Theorem 4.23, $[G]$ is reflexive and the theorem is proved. Otherwise, we execute steps 8 and 9. Let $\mathcal{I}_{1}=[F], L_{1}=(\mathbb{f}), \mathcal{I}_{2}=\left[G \cup\left\{\mathbb{Y}^{\mathbf{h}_{i}}-\sigma^{-k_{i}}\left(\prod_{j=1}^{s} d_{j}^{b_{i, j}}\right), i=1, \ldots, t\right\}\right]$, and $L_{2}=\mathbb{L}\left(\mathcal{I}_{2}\right)$. Then, we have $\mathcal{I}_{1} \varsubsetneqq \mathcal{I}_{2} \subset \mathcal{I}_{x}$ and $L_{1} \varsubsetneqq L_{2} \subset L_{x}$, where $L_{x}=$ sat $_{x}\left(L_{1}\right)$ and $\mathcal{I}_{x}$ is the reflexive closure of $\mathcal{I}_{1}$. Similar to the proof of Theorem 4.26, the algorithm will terminate and output the reflexive closure of $[P]$.

Remark 7.20 Following Algorithm 5, we can give an algorithm to check whether a Laurent binomial $\sigma$-ideal is well-mixed or perfect, and in the negative case to compute the well-mixed or perfect closure of the $\sigma$-ideal. The details are omitted.

We give a decomposition algorithm for Laurent binomial $\sigma$-ideals. 


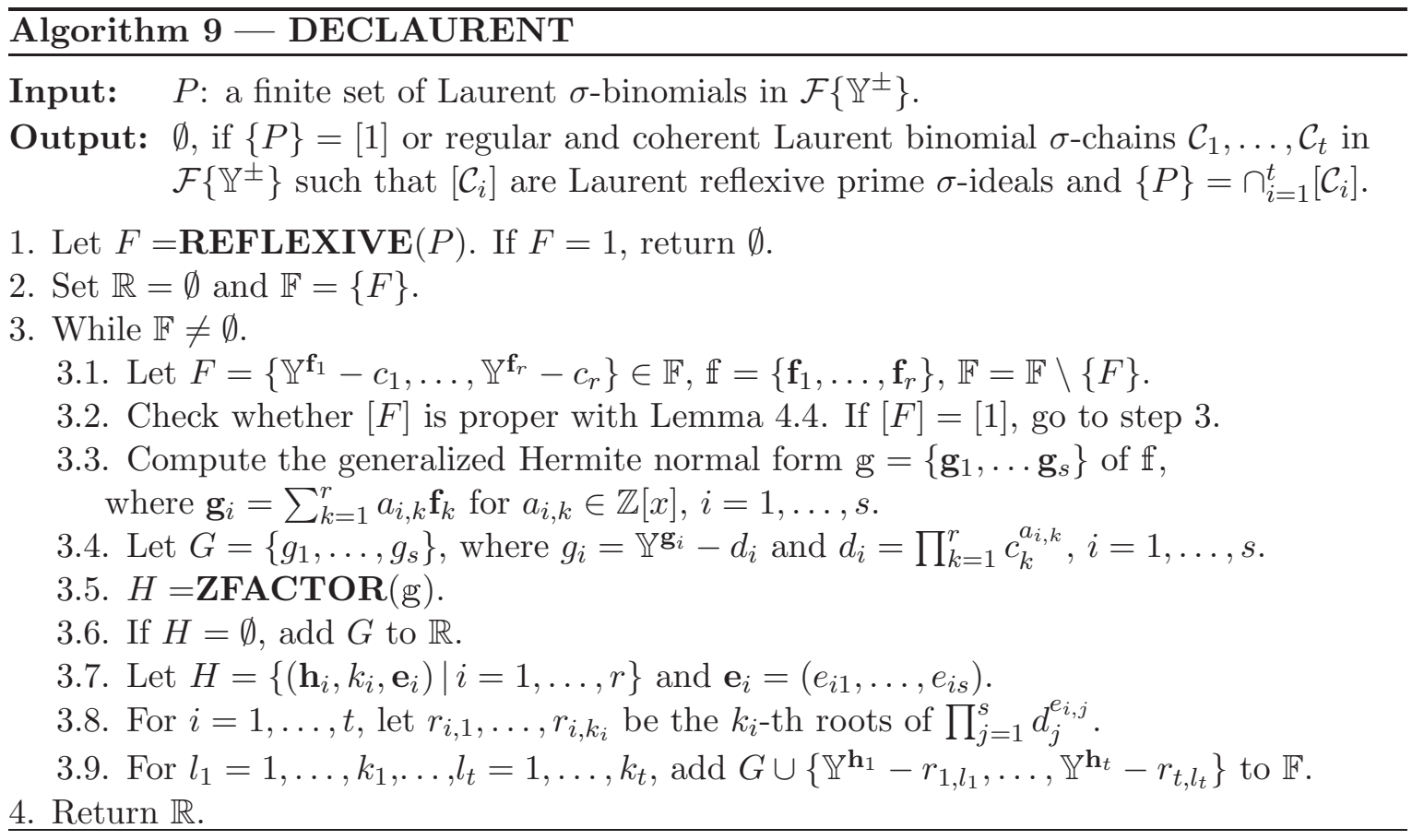

Theorem 7.21 Algorithm DECLAURENT is correct.

Proof: The algorithm basically follows the proof of Theorem 4.29. The proof is similar to that of Theorem 7.19 .

In the rest of this section, we give a decomposition algorithm for binomial $\sigma$-ideals and hence a proof for Theorem 1.4. Before giving the main algorithm, we give a sub-algorithm DECMONO which treats the $\sigma$-monomials. Basically, it gives the following decomposition

$$
\mathbb{V}\left(\prod_{i=1}^{n} y_{i}^{f_{i}}\right)=\mathbb{V}\left(y_{1}\right) \cup \mathbb{V}\left(y_{2} / y_{1}\right) \cup \cdots \cup \mathbb{V}\left(y_{n} /\left\{y_{1}, \ldots, y_{n-1}\right\}\right)
$$

where $0 \neq f_{i} \in \mathbb{N}[x]$ and $\mathbb{V}\left(y_{c} / S\right)$ is the set of zeros of $y_{c}=0$ not vanishing any of the variables in $S$. The correctness of the algorithm comes directly from the above formula. 


\section{Algorithm $10-$ DECMONO}

Input: $\quad\left(\mathbb{Y}_{0}, B, \mathbb{Y}_{1}\right): \mathbb{Y}_{0}, \mathbb{Y}_{1}$ are disjoint subsets of $\mathbb{Y}$ and $B$ a finite set of $\sigma$-binomials in $\mathcal{F}\{\mathbb{Y}\}$.

Output: $\left(\mathbb{Y}_{0 i}, B_{i}, \mathbb{Y}_{1 i}\right): \mathbb{Y}_{0 i}, \mathbb{Y}_{1 i}$ are disjoint subsets of $\mathbb{Y}, B_{i}$ contains no $\sigma$-monomials, and $\mathbb{V}\left(\mathbb{Y}_{0} \cup B / \mathbb{Y}_{1}\right)=\cup_{i=1}^{r} \mathbb{V}\left(\mathbb{Y}_{0 i} \cup B_{i} / \mathbb{Y}_{1 i}\right)$.

1. Set $\mathbb{R}=\emptyset$ and $\mathbb{F}=\left\{\left(\mathbb{Y}_{0}, B, \mathbb{Y}_{1}\right)\right\}$.

2. While $\mathbb{F} \neq \emptyset$.

2.1. Let $C=\left(\mathbb{Y}_{0}, B, \mathbb{Y}_{1}\right) \in \mathbb{F}, \mathbb{F}=\mathbb{F} \backslash\{C\}$.

2.2. For all $y_{c} \in \mathbb{Y}_{0}$, let $B_{1}=B_{y_{c}=0}$ (replace $y_{c}$ by 0 ) and delete 0 from $B_{1}$.

2.3. If $B_{1}$ contains no $\sigma$-monomials, add $\left(\mathbb{Y}_{0}, B_{1}, \mathbb{Y}_{1}\right)$ to $\mathbb{R}$ and goto step 2 .

2.4. Let $M=\prod_{i=1}^{k} y_{c_{i}}^{f_{i}} \in B_{1}$, where $0 \neq f_{i} \in \mathbb{N}[x] . B_{1}=B_{1} \backslash\{M\}$.

2.5. Let $\mathbb{Y}_{2}:=\left\{y_{c_{1}}, \ldots, y_{c_{k}}\right\} \backslash \mathbb{Y}_{1}$. If $\mathbb{Y}_{2}=\emptyset$, go to step 2 ; else let $\mathbb{Y}_{2}=\left\{y_{t_{1}}, \ldots, y_{t_{s}}\right\}$.

2.6. For $i=1, \ldots, s$, add $\left(\mathbb{Y}_{0} \cup\left\{y_{t_{i}}\right\}, B_{1}, \mathbb{Y}_{1} \cup\left\{y_{t_{1}}, \ldots, y_{t_{i-1}}\right\}\right)$ to $\mathbb{F}$.

3. Return $\mathbb{R}$.

We now give the main algorithm. The algorithm basically follows the proof of Theorem 5.28. The main modification is that instead of the perfect ideal decomposition

$$
\{F\}=(\{F\}: \mathrm{m}) \bigcap \cap_{i=1}^{n}\left\{F, y_{i}\right\},
$$

we use the following zero decomposition

$$
\mathbb{V}(F)=\mathbb{V}(\{F\}: \mathbb{m}) \bigcup \cup_{i=1}^{n} \mathbb{V}\left(F \cup\left\{y_{i}\right\} /\left\{y_{1}, \ldots, y_{i-1}\right\}\right) .
$$

The purpose of using the later decomposition is that many redundant components can be easily removed by the following criterion $\mathbb{V}(F / D)=\emptyset$ if $F \cap D \neq \emptyset$, which is done in step 2.5 of Algorithm DECMONO.

\section{Algorithm 11 - DECBINOMIAL}

Input: $\quad F$ : a finite set of $\sigma$-binomials in $\mathcal{F}\{\mathbb{Y}\}$.

Output: $\emptyset$, if $\{F\}=[1]$ or $\left(\mathcal{C}_{1}, \mathbb{Y}_{1}\right), \ldots,\left(\mathcal{C}_{r}, \mathbb{Y}_{r}\right)$, where $\mathbb{Y}_{i} \subset \mathbb{Y}$ and $\mathcal{C}_{i}$ are regular and coherent $\sigma$-chains containing $\sigma$-binomials of variables in $\mathbb{Y} \backslash \mathbb{Y}_{i}$ such that $\operatorname{sat}\left(\mathcal{C}_{i}\right)$ are reflexive prime $\sigma$-ideals and $\{F\}=\cap_{i=1}^{r} \operatorname{sat}\left(\mathcal{C}_{i}\right)$.

1. Set $\mathbb{R}=\emptyset$ and $\mathbb{F}=\operatorname{DECMONO}(\emptyset, F, \emptyset)$.

2. While $\mathbb{F} \neq \emptyset$.

2.1. Let $C=\left(\mathbb{Y}_{0}, B, \mathbb{Y}_{1}\right) \in \mathbb{F}, \mathbb{F}=\mathbb{F} \backslash\{C\}$.

2.2. If $B=\emptyset$, add $\left(\mathbb{Y}_{0}, \mathbb{Y}_{1}\right)$ to $\mathbb{R}$.

2.3. Let $E=\operatorname{DECLAUENT}(B)$ in $\mathcal{F}\left\{\mathbb{Z}^{ \pm}\right\}$, where $\mathbb{Z}=\mathbb{Y} \backslash \mathbb{Y}_{0}$ and $m=|\mathbb{Z}|$.

2.4. If $E=\emptyset$ goto step2.

2.5. Let $E=\left\{E_{1}, \ldots, E_{l}\right\}$ and $E_{l}=\left\{\mathbb{Z}^{\mathbf{f}_{l, 1}}-c_{l, 1}, \ldots, \mathbb{Z}^{\mathbf{f}_{l, s_{l}}}-c_{l, s_{l}}\right\}$, where $\mathbf{f}_{l, k} \in \mathbb{Z}[x]^{m}$.

2.7. Add $\left(\left\{\mathbb{Y}_{0}, \mathbb{Z}^{\mathbf{f}_{l, 1}^{+}}-c_{l, 1} \mathbb{Z}^{\mathbf{f}_{l, 1}^{-}}, \ldots, \mathbb{Z}^{\mathbf{f}_{l, s_{l}}^{+}}-c_{l, s_{l}} \mathbb{Z}^{\mathbf{f}_{l, s_{l}}^{-}}\right\}, \mathbb{Y}_{1}\right)$ to $\mathbb{R}, l=1, \ldots, k$.

2.8. Let $\mathbb{Z}=\left\{y_{c_{1}}, \ldots, y_{c_{s}}\right\}$. For $i=1, \ldots, s$, do

$$
\mathbb{F}=\mathbb{F} \cup \operatorname{DECMONO}\left(\mathbb{Y}_{0} \cup\left\{y_{c_{i}}\right\}, B, \mathbb{Y}_{1} \cup\left\{y_{c_{1}}, \ldots, y_{c_{i-1}}\right\}\right) .
$$

3. Return $\mathbb{R}$. 
Theorem 7.22 Algorithm DECBINOMIAL is correct.

Proof: In step $1, \mathbb{V}(F)$ is decomposed as $\mathbb{V}(F)=\cup_{i=1}^{r} \mathbb{V}\left(\mathbb{Y}_{0 i} \cup B_{i} / \mathbb{Y}_{1 i}\right)$ and $\mathbb{F}=\left\{\left(\mathbb{Y}_{0 i}, B_{i}\right.\right.$, $\left.\left.\mathbb{Y}_{1 i}\right) ; i=1, \ldots, r\right\}$. In step 2 , we will treat the components of $\mathbb{F}$ one by one. In step 2.1 , the component $\left(\mathbb{Y}_{0}, B, \mathbb{Y}_{1}\right)$ is taken from $\mathbb{F}$. In steps 2.3-2.4, $\{B\}$ is decomposed as

$$
\{B\}=\cap_{l=1}^{k}\left[E_{l}\right]
$$

in $\mathcal{F}\left\{\mathbb{Z}^{ \pm}\right\}$, where $E_{l}$ are regular and coherent $\sigma$-chains and $\left[E_{l}\right]$ are reflexive prime ideals. By (27) and Corollary 5.37, we have

$$
\{B\}: \mathrm{m}=\{B\} \cap \mathcal{F}\{\mathbb{Z}\}=\cap_{l=1}^{k}\left(\left[E_{l}\right] \mathcal{F}\left\{\mathbb{Z}^{ \pm}\right\}\right) \cap \mathcal{F}\{\mathbb{Y}\}=\cap_{l=1}^{k} \operatorname{sat}\left(E_{l}^{+}\right),
$$

where $E_{l}^{+}=\left\{\mathbb{Z}^{\mathbf{f}_{l, 1}^{+}}-c_{l, 1} \mathbb{Z}^{\mathbf{f}_{l, 1}^{-}}, \ldots, \mathbb{Z}^{\mathbf{f}_{l, s_{l}}^{+}}-c_{l, s_{l}} \mathbb{Z}^{\mathbf{f}_{l, s_{l}}^{-}}\right\}, l=1, \ldots, k$. Since $E_{l}$ is regular and coherent, by Lemma 5.34, $E_{l}^{+}$is also regular and coherent.

Since $B \subset \mathcal{F}\{\mathbb{Z}\}$, we have the following zero decomposition

$$
\mathbb{V}\left(\mathbb{Y}_{0} \cup B / \mathbb{Y}_{1}\right)=\mathbb{V}\left(\mathbb{Y}_{0} \cup(\{B\}: \mathrm{m}) / \mathbb{Y}_{1}\right) \cup_{i=1}^{s} \mathbb{V}\left(\mathbb{Y}_{0} \cup B \cup\left\{y_{c_{i}}\right\} /\left\{y_{c_{1}}, \ldots, y_{c_{i-1}}\right\} \cup \mathbb{Y}_{1}\right),
$$

where $\mathbb{V}\left(\mathbb{Y}_{0} \cup B \cup\left\{y_{c_{i}}\right\} /\left\{y_{c_{1}}, \ldots, y_{c_{i-1}}\right\} \cup \mathbb{Y}_{1}\right)$ is further simplified with algorithm DECMONO in step 2.8. From (44),

$$
\mathbb{V}\left(\mathbb{Y}_{0} \cup\{B\}: \mathrm{m} / \mathbb{Y}_{1}\right)=\cup_{l=1}^{k} \mathbb{V}\left(\operatorname{sat}\left(\mathbb{Y}_{0}, E_{l}^{+}\right) / \mathbb{Y}_{1}\right)=\cup_{l=1}^{k} \mathbb{V}\left(\left[\mathbb{Y}_{0}, \operatorname{sat}\left(E_{l}^{+}\right)\right] / \mathbb{Y}_{1}\right),
$$

where $\left\{\mathbb{Y}_{0}, E_{l}^{+}\right\}$is a regular and coherent $\sigma$-chain since $E_{l}^{+}$does not contain variables in $\mathbb{Y}_{0}$. The above formula explains why $\left(\left\{\mathbb{Y}_{0}, E_{l}^{+}\right\}, \mathbb{Y}_{1}\right)$ is added to $\mathbb{R}$ in steps 2.5-2.7.

Let the algorithm returns $\mathbb{R}=\left\{\left(\mathcal{C}_{i}, \mathbb{Y}_{i}\right) ; i=1, \ldots, m\right\}$. From the above proof, we have $\mathbb{V}(F)=\cup_{l=1}^{k} \mathbb{V}\left(\operatorname{sat}\left(\mathcal{C}_{i}\right) / \mathbb{Y}_{i}\right)$. Since $\mathbb{Y}_{i} \cap \mathcal{C}_{i}=\emptyset$ and $\operatorname{sat}\left(\mathcal{C}_{i}\right)$ is a prime $\sigma$-ideal, the Cohn closure of $\mathbb{V}\left(\operatorname{sat}\left(\mathcal{C}_{i}\right) / \mathbb{Y}_{i}\right)$ is $\mathbb{V}\left(\operatorname{sat}\left(\mathcal{C}_{i}\right)\right)$ and hence

$$
\mathbb{V}(F)=\cup_{l=1}^{k} \mathbb{V}\left(\operatorname{sat}\left(\mathcal{C}_{i}\right) / \mathbb{Y}_{i}\right)=\cup_{l=1}^{k} \mathbb{V}\left(\operatorname{sat}\left(\mathcal{C}_{i}\right)\right) .
$$

By the difference Hilbert Nullstellensatz,

$$
\{F\}=\cap_{l=1}^{k}\left\{\operatorname{sat}\left(\mathcal{C}_{i}\right)\right\}=\cap_{l=1}^{k} \operatorname{sat}\left(\mathcal{C}_{i}\right) .
$$

The algorithm terminates, since after each execution of step 2, in the new components $\left(\mathbb{Y}_{0 l}, B_{l}, \mathbb{Y}_{1 l}\right)$ added to $\mathbb{F}$ in step $2.8, B_{l}$ contains at least one less variables than $B$.

The following example shows that even in the binomial case, a proper irreducible $\sigma$-chain is not necessarily strong irreducible [10].

Example 7.23 Let $\mathcal{A}=\left\{A_{1}, A_{2}, A_{3}\right\}$ be a proper irreducible $\sigma$-chian, where $A_{1}=y_{1}^{x^{k}}-y_{1}^{2}$, $A_{2}=y_{2}^{x^{k}}-y_{2}^{2}, A_{3}=y_{1} y_{3}^{3 x}-y_{2}^{x}$, and $k \geq 2$. Then $A_{3}^{x^{k}}=y_{1}^{2} y_{3}^{3(x+k)}-y_{2}^{2 x} \bmod \left[A_{1}, A_{2}\right]$. Thus $A_{3}^{x^{k}}-\left(y_{1} y_{3}^{3 x}+y_{2}^{x}\right) A_{3}=y_{1}^{2}\left(y_{3}^{3(x+k)}-y_{3}^{3 x}\right) \bmod \left[A_{1}, A_{2}\right]$ is reducible and $\mathcal{A}$ is not strong irreducible.

Remark 7.24 It is still open to give a minimal decomposition for finitely generated binomial $\sigma$-ideals. Or equivalently, the Ritt problem [19, $p$ 191] is open even for binomial $\sigma$-chains. 


\section{Conclusion}

In this paper, we initiate the study of binomial $\sigma$-ideals and toric $\sigma$-varieties. Two basic tools used are the $\mathbb{Z}[x]$-lattice and the characteristic set instead of the $\mathbb{Z}$-lattice and the Gröbner basis used in the algebraic case. Since $\mathbb{Z}[x]$ is not a PID, a matrix with entries in $\mathbb{Z}[x]$ does not have a Hermite normal form. As an alternative, we introduce the concept of generalized Hermite normal form which is equivalent to a reduced Gröbner basis for $\mathbb{Z}[x]$-lattices. It is shown that a set of Laurent $\sigma$-binomials is a regular and coherent $\sigma$-chain if and only if their supports form a generalized Hermite normal form.

For Laurent binomial $\sigma$-ideals, three main results are proved. Canonical representations for proper Laurent binomial $\sigma$-ideals are given in terms of Gröbner basis of $\mathbb{Z}[x]$-lattices, regular and coherent $\sigma$-chains in $\mathcal{F}\left\{\mathbb{Y}^{ \pm}\right\}$, and partial characters over $\mathbb{Z}[x]^{n}$. We also give criteria for a Laurent binomial $\sigma$-ideal to be reflexive, well-mixed, perfect, and prime in terms of its support lattice. It is also shown that the reflexive, well-mixed, and perfect closure of a Laurent binomial $\sigma$-ideal is still binomial. Finally, it is shown that a perfect Laurent $\sigma$-ideal can be written as the intersection of Laurent reflexive prime binomial $\sigma$-ideals with the same support lattice. Most of these results are also extended to the $\sigma$-binomial case.

A toric $\sigma$-variety is defined as the Cohn closure of the image of Laurent $\sigma$-monomial maps. Three characterizing properties of toric $\sigma$-varieties are proved in terms of its coordinate ring, its defining ideals, and group actions. In particular, a $\sigma$-variety is toric if and only if its defining ideal is a toric $\sigma$-ideal, meaning a binomial $\sigma$-ideal whose support lattice is $\mathbb{Z}[x]$ saturated.

Finally, algorithms are given for all the main results in the paper, that is, to decide whether a finitely generated Laurent binomial $\sigma$-ideal is reflexive, well-mixed, perfect, prime, or toric, and to decompose a finitely generated perfect binomial $\sigma$-ideal as intersection of reflexive prime binomial $\sigma$-ideals.

\section{References}

[1] D. Bouziane, A. Kandri Rody, H. Maârouf. Unmixed-dimensional Decomposition of a Finitely Generated Perfect Differential Ideal. Journal of Symbolic Computation, 31(6), 631-649, 2001.

[2] H. Cohen. A Course in Computational Algebraic Number Theory. Springer-Verlag, Berlin, 1993.

[3] R. M. Cohn. Difference Algebra. Interscience Publishers, New York, 1965.

[4] D. Cox, J. Little, D. O'Shea. Using Algebraic Geometry, Springer-Verlag, New York, 1998.

[5] D. Cox, J. Little, H. Schenck. Toric Varieties, Springer-Verlag, New York, 2010.

[6] M. Demazure. Sous-groupes Algébriques de Rang Maximum du Groupe de Cremona, Ann. Sci. École Norm. Sup., 3, 507-588, 1970. 
[7] D. Eisenbud and B. Sturmfels. Binomial Ideals. Duke Math. J., 84(1), 1-45, 1996.

[8] W. Fulton. Introduction to Toric Varieties, Princeton Univ. Press, Princeton, USA, 1993.

[9] X. S. Gao, W. Li, C. M. Yuan. Intersection Theory in Differential Algebraic Geometry: Generic Intersections and the Differential Chow Form. Trans. of Amer. Math. Soc., 365(9), 4575-4632, 2013.

[10] X. S. Gao, Y. Luo, C. M. Yuan. A Characteristic Set Method for Ordinary Difference Polynomial Systems. Journal of Symbolic Computation, 44(3), 242-260, 2009.

[11] X. S. Gao, C. Yuan, and G. Zhang. Ritt-Wu's Characteristic Set Method for Ordinary Difference Polynomial Systems with Arbitrary Ordering. Acta Mathematica Scientia, 29(3,4), 1063-1080, 2009.

[12] X. S. Gao and S. C. Chou. Implicitization of Rational Parametric Equations. Journal of Symbolic Computation, 14, 459-470, 1992.

[13] I. M. Gelfand, M. Kapranov, A. Zelevinsky. Discriminants, Resultants and Multidimensional Determinants. Boston, Birkhäuser, 1994.

[14] K. O. Geddes, S. R. Czapor, G. Labahn. Algorithms for Computer Algebra. Kluwer Academic Publishers, Boston, MA, 1992.

[15] E. Hrushovski. The Elementary Theory of the Frobenius Automorphisms. Available from http://www.ma.huji.ac.il/ ehud/, July, 2012.

[16] K. I. Iima and Y. Yoshino. Gröbner Bases for the Polynomial Ring with Infinite Variables and Their Applications, Communications in Algebra, 37:10, 3424-3437, 2009.

[17] A. Kandri Rody and D. Kapur. Computing a Gröbner Basis of a Polynomial Ideal over a Euclidean Domain. Journal of Symbolic Computation, 6(1), 37-57, 1988.

[18] G. Kempf, F. Knudsen, D. Mumford, B. Saint-Donat. Toroidal Embeddings I, Lecture Notes in Math. 339, Springer-Verlag, Berlin, 1973.

[19] E. R. Kolchin. Differential Algebra and Algebraic Groups. Academic Press, New York and London, 1973.

[20] A. Levin. Difference Algebra. Springer-Verlag, New Work, 2008.

[21] W. Li, C. M. Yuan, X. S. Gao. Sparse Difference Resultant. Proc. ISSAC 2013, 275-282, ACM Press, New York, 2013.

[22] W. Li, C. M. Yuan, X. S. Gao. Sparse Difference Resultant. Journal of Symbolic Computation, 68, 169-203, 2015.

[23] W. Li, C. M. Yuan, X. S. Gao. Sparse Differential Resultant for Laurent Differential Polynomials. Foundations of Computational Mathematics, 15(2), 451-517, 2015. 
[24] W. Li and Y. H. Li. Difference Chow Form. Journal of Algebra, 428, 67-90, 2015.

[25] A. Medvedev and T. Scanlon. Invariant Varieties for Polynomial Dynamical Systems. Annals of Mathematics, 179(1), 181-177, 2014.

[26] E. Miller and B. Sturmfels. Combinatorial Commutative Algebra. Springer-Verlag, New York, 2005.

[27] K. Miyake and T. Oda. Almost Homogeneous Algebraic Varieties under Algebraic Torus Action, in Manifolds-Tokyo, (A. Hattori, ed.), 373-381, Univ. Tokyo Press, Tokyo, 1975.

[28] T. Oda. Convex Bodies and Algebraic Geometry. Springer, New York, 1988.

[29] L. Pachter and B. Sturmfels (eds). Algebraic Statistics for Computational Biology. Cambridge University Press, 2005.

[30] J. F. Ritt and J. L. Doob. Systems of Algebraic Difference Equations. American Journal of Mathematics, 55(1), 505-514, 1933.

[31] I. Satake. On the Arithmetic of Tube Domains (blowing-up of the point at infinity), Bull. Amer. Math. Soc. 79, 1076-1094, 1973.

[32] M. van der Put and M. F. Singer. Galois Theory of Difference Equations, Springer, Berlin and New York, 1997.

[33] M. Wibmer. Algebraic Difference Equations. Preprint, 2013. 